\title{
Theory of Fundamental Bessel Functions of High Rank
}

\author{
Zhi Qi
}

Author address:

Department of Mathematics, The Ohio State University, 100 Math Tower, 231 West 18th Avenue, Columbus, OH 43210, USA

E-mail address: qi .91@buckeyemail.osu.edu 

To Hui 



\section{Contents}

Introduction

Notation

Chapter 1. Hankel Transforms and Bessel Kernels 1

1. Preliminaries 1

2. The Function Spaces $\mathscr{S}_{\text {sis }}\left(\mathbb{R}_{+}\right), \mathscr{S}_{\text {sis }}\left(\mathbb{R}^{\times}\right)$and $\mathscr{S}_{\text {sis }}\left(\mathbb{C}^{\times}\right)$

3. Hankel Transforms and Bessel Kernels 17

4. Bessel Functions and Bessel Kernels in Classical Cases 29

5. Fourier Type Integral Transforms 31

6. Integral Representations of Bessel Kernels 37

A. A Prototypical Example of Bessel Functions 46

Chapter 2. Analytic Theory of Bessel Functions 49

7. The Rigorous Interpretation of the Formal Integral $J_{v}(x ; \boldsymbol{\varsigma})$

8. Equality between $J_{v}(x ; \boldsymbol{\varsigma})$ and $J(x ; \boldsymbol{\varsigma}, \lambda)$

9. $H$-Bessel Functions and $K$-Bessel Functions

10. Recurrence Formulae and the Differential Equations for Bessel Functions 61

11. Bessel Equations 66

12. Connections between Various Types of Bessel Functions 81

13. $H$-Bessel Functions and $K$-Bessel Functions, II 83

B. An Alternative Approach to Asymptotic Expansions 84

Chapter 3. Bessel Kernels 87

14. The Asymptotic of $J_{(\lambda, \delta)}(x)$

15. Two Connection Formulae for $J_{(\mu, m)}(z)$

16. The Asymptotic Expansion of $J_{(\mu, m)}(z)$

Chapter 4. Hankel Transforms and Bessel Kernels in Representation Theory 97

17. Hankel Transforms from the Representation Theoretic Viewpoint 97

18. Bessel Functions for $\mathrm{GL}_{2}(\mathbb{F}) \quad 102$

Bibliography 105 



\begin{abstract}
In this article, we shall study fundamental Bessel functions for $\mathrm{GL}_{n}(\mathbb{F})$ arising from the Voronoï summation formula for any rank $n$ and field $\mathbb{F}=\mathbb{R}$ or $\mathbb{C}$, with focus on developing their analytic and asymptotic theory. The main implements and subjects of our study of fundamental Bessel functions are their formal integral representations and Bessel differential equations. We shall prove the asymptotic formulae for fundamental Bessel functions and explicit connection formulae for the Bessel differential equations.
\end{abstract}

2010 Mathematics Subject Classification. 33E20, 33E30, 44A20.

Key words and phrases. Hankel transforms, Bessel kernels, Bessel functions, formal integral representations, Bessel differential equations. 



\section{Introduction}

\section{Number Theoretic Motivations}

The oscillatory exponential function $e(x)=e^{2 \pi i x}$ arises as the integral kernel of the Fourier transform in Poisson's summation formula, which has played a very important role in analysis and number theory. The Poisson summation asserts the identity

$$
\sum_{n=-\infty}^{\infty} v(n)=\sum_{n=-\infty}^{\infty} \widehat{v}(n)
$$

for at least all Schwartz functions $v$, with $\widehat{v}$ the Fourier transform of $v$,

$$
\widehat{v}(x)=\int_{-\infty}^{\infty} v(y) e(-x y) d y .
$$

Riemann's proof of the functional equation of his zeta function relies on the Poisson summation formula. Also, Hecke used the higher dimensional generalization of the formula for the zeta function associated with an arbitrary number field. Furthermore, Tate's thesis reinterprets these using the Poisson summation formula for the adele ring.

(Classical) Bessel functions occur in Voronoï's summation formula as well as Petesson's and Kuznetsov's trace formula for $\mathrm{GL}_{2}(\mathbb{R})$. These formulae have become fundamental analytic tools for a number of deep results in analytic number theory, most notably for the subconvexity problem for automorphic $L$-functions. A version of the Voronoï summation formula, which is not in the most general form, reads as follows (see for example [KMV, Theorem A.4] and [HM Proposition 1]),

$$
\sum_{n=1}^{\infty} \sqrt{n} \rho_{F}^{+}(n) e\left(\frac{a n}{c}\right) v(n)=\frac{1}{c} \sum_{ \pm} \sum_{n=1}^{\infty} \sqrt{n} \rho_{F}^{ \pm}(n) e\left(\mp \frac{\bar{a} n}{c}\right) \Upsilon\left( \pm \frac{n}{c^{2}}\right) .
$$

In this formula, $a, \bar{a}$ and $c$ are integers such that $(a, c)=1$ and $a \bar{a} \equiv 1(\bmod c), \rho_{F}^{ \pm}(n)$ are certain normalized Fourier coefficients of a holomprhic or Maaß cusp form for $\mathrm{SL}_{2}(\mathbb{Z}), v$ is a smooth weight function compactly supported on $(0, \infty)$ and $\Upsilon$ is the Hankel transform of $v$,

$$
\Upsilon(x)=\int_{0}^{\infty} v(y) J_{F}(x y) d y, \quad x \neq 0
$$

where, if $F$ is a Maaß form of eigenvalue $\frac{1}{4}+t^{2}$ and weight $k$,

$$
\begin{aligned}
J_{F}(x) & =-\frac{\pi}{\cosh (\pi t)}\left(Y_{2 i t}(4 \pi \sqrt{x})+Y_{-2 i t}(4 \pi \sqrt{x})\right) \\
& =\frac{\pi i}{\sinh (\pi t)}\left(J_{2 i t}(4 \pi \sqrt{x})-J_{-2 i t}(4 \pi \sqrt{x})\right) \\
& =\pi i\left(e^{-\pi t} H_{2 i t}^{(1)}(4 \pi \sqrt{x})-e^{\pi t} H_{2 i t}^{(2)}(4 \pi \sqrt{x})\right),
\end{aligned}
$$




$$
\begin{aligned}
J_{F}(-x) & =4 \cosh (\pi t) K_{2 i t}(4 \pi \sqrt{x}) \\
& =\frac{\pi i}{\sinh (\pi t)}\left(I_{2 i t}(4 \pi \sqrt{x})-I_{-2 i t}(4 \pi \sqrt{x})\right), \quad x>0,
\end{aligned}
$$

for $k$ even,

$$
\begin{aligned}
J_{F}(x) & =-\frac{\pi}{\sinh (\pi t)}\left(Y_{2 i t}(4 \pi \sqrt{x})-Y_{-2 i t}(4 \pi \sqrt{x})\right) \\
& =\frac{\pi i}{\cosh (\pi t)}\left(J_{2 i t}(4 \pi \sqrt{x})+J_{-2 i t}(4 \pi \sqrt{x})\right) \\
& =\pi i\left(e^{-\pi t} H_{2 i t}^{(1)}(4 \pi \sqrt{x})+e^{\pi t} H_{2 i t}^{(2)}(4 \pi \sqrt{x})\right) \\
J_{F}(-x) & =4 \sinh (\pi t) K_{2 i t}(4 \pi \sqrt{x}) \\
& =\frac{\pi i}{\cosh (\pi t)}\left(I_{2 i t}(4 \pi \sqrt{x})-I_{-2 i t}(4 \pi \sqrt{x})\right), \quad x>0,
\end{aligned}
$$

for $k$ odd, and if $F$ is a holomorphic cusp form of weight $k$,

$$
J_{F}(x)=2 \pi i^{k} J_{k-1}(4 \pi \sqrt{x}), \quad J_{F}(-x)=0, \quad x>0 .
$$

Thus the integral kernel $J_{F}$ has an expression in Bessel functions, where, in standard notation, $J_{v}, Y_{v}, H_{v}^{(1)}, H_{v}^{(2)}, I_{v}$ and $K_{v}$ are the various Bessel functions (see for instance [Wat]). Here, the following connection formulae ([Wat $3.61(3,4,5,6), 3.7$ (6)]) have been applied in 0.1$)$ and $(0.2)$,

$$
\begin{array}{ll}
Y_{v}(x)=\frac{J_{v}(x) \cos (\pi v)-J_{-v}(x)}{\sin (\pi v)}, & Y_{-v}(x)=\frac{J_{v}(x)-J_{-v}(x) \cos (\pi v)}{\sin (\pi v)}, \\
H_{v}^{(1)}(x)=\frac{J_{-v}(x)-e^{-\pi i v} J_{v}(x)}{i \sin (\pi v)}, & H_{v}^{(2)}(x)=\frac{e^{\pi i v} J_{v}(x)-J_{-v}(x)}{i \sin (\pi v)}, \\
K_{v}(x)=\frac{\pi\left(I_{-v}(x)-I_{v}(x)\right)}{2 \sin (\pi v)} . &
\end{array}
$$

The theory of Bessel functions has been extensively studied since the early 19th century, and we refer the reader to Watson's beautiful book [Wat] for an encyclopedic treatment.

The Voronoï summation formula for $\mathrm{GL}_{n}(\mathbb{Z})$ with $n \geqslant 3$ is formulated in the work of Miller and Schmid [MS3, MS4] (see also [GL1, GL2]), in which Hankel transforms are the Archimedean ingredient that relates the weight functions on two sides of the identity. The notion of automorphic distributions is used for their proof of this formula, and is also used to derive the analytic continuation and the functional equation of the $L$-function of a cuspidal $\mathrm{GL}_{n}(\mathbb{Z})$-automorphic representation of $\mathrm{GL}_{n}(\mathbb{R})$. As the foundation of automorphic distributions, the harmonic analysis over $\mathbb{R}$ is studied in [MS3] from the viewpoint of gamma factors and signed Mellin transforms. As explained in [MS2], the cases $n=1,2$ can also be incorporated into their framework.

More recently, using the global theory of $\mathrm{GL}_{n} \times \mathrm{GL}_{1}$-Rankin-Selberg $L$-functions, Inchino and Templier [IT] extended Miller and Schmid's work and proved the Voronoï summation formula for any irreducible cuspidal automorphic representation of $\mathrm{GL}_{n}$ over an arbitrary number field for $n \geqslant 2$. According to [IT], the associated Hankel transform over an Archimedean local field is obtained from the corresponding local functional equations for $\mathrm{GL}_{n} \times \mathrm{GL}_{1}$-Rankin-Selberg zeta integrals over the field.

In order to motivate our study, let us give some detailed descriptions of Hankel transforms of any rank over the real numbers in [MS3, MS4]. 
Suppose that $(\boldsymbol{\lambda}, \boldsymbol{\delta})=\left(\lambda_{1}, \ldots, \lambda_{n}, \delta_{1}, \ldots, \delta_{n}\right) \in \mathbb{C}^{n} \times(\mathbb{Z} / 2 \mathbb{Z})^{n}$ is a certain parameter of a cuspidal $\mathrm{GL}_{n}(\mathbb{Z})$-automorphic representation of $\mathrm{GL}_{n}(\mathbb{R})$. Miller and Schmid give two expressions for the associated Hankel transform.

The first expression of the Hankel transform associated with $(\boldsymbol{\lambda}, \boldsymbol{\delta})$ is based on gamma factors and signed Mellin transforms as follows.

Let $\mathscr{S}(\mathbb{R})$ denote the space of Schwartz functions on $\mathbb{R}$. For $\lambda \in \mathbb{C}, j \in \mathbb{N}=$ $\{0,1,2, \ldots\}$ and $\eta \in \mathbb{Z} / 2 \mathbb{Z}=\{0,1\}$, let $v$ be a smooth function on $\mathbb{R}^{\times}=\mathbb{R} \backslash\{0\}$ such that $\operatorname{sgn}(x)^{\eta}|x|^{\lambda}(\log |x|)^{-j} v(x) \in \mathscr{S}(\mathbb{R})$. For $\delta \in \mathbb{Z} / 2 \mathbb{Z}$, the signed Mellin transform $\mathcal{M}_{\delta} v$ with order $\delta$ of $v$ is defined by

$$
\mathcal{M}_{\delta} v(s)=\int_{\mathbb{R}^{\times}} v(x) \operatorname{sgn}(x)^{\delta}|x|^{s} d^{\times} x .
$$

Here $d^{\times} x=|x|^{-1} d x$ is the standard multiplicative Haar measure on $\mathbb{R}^{\times}$. The Mellin inversion formula is

$$
v(x)=\sum_{\delta \in \mathbb{Z} / 2 \mathbb{Z}} \frac{\operatorname{sgn}(x)^{\delta}}{4 \pi i} \int_{(\sigma)} \mathcal{M}_{\delta} v(s)|x|^{-s} d s, \quad \sigma>\Re \mathrm{e} \lambda,
$$

where the contour of integration $(\sigma)$ is the vertical line from $\sigma-i \infty$ to $\sigma+i \infty$.

Let $\mathscr{S}\left(\mathbb{R}^{\times}\right)$denote the space of smooth functions on $\mathbb{R}^{\times}$whose derivatives are rapidly decreasing at both zero and infinity. We associate with $v \in \mathscr{S}\left(\mathbb{R}^{\times}\right)$a function $\Upsilon$ on $\mathbb{R}^{\times}$ satisfying the following two identities

$$
\mathcal{M}_{\delta} \Upsilon(s)=\left(\prod_{l=1}^{n} G_{\delta_{l}+\delta}\left(s-\lambda_{l}\right)\right) \mathcal{M}_{\delta} v(1-s), \quad \delta \in \mathbb{Z} / 2 \mathbb{Z},
$$

where $G_{\delta}(s)$ denotes the gamma factor

$$
G_{\delta}(s)=i^{\delta} \pi^{\frac{1}{2}-s} \frac{\Gamma\left(\frac{1}{2}(s+\delta)\right)}{\Gamma\left(\frac{1}{2}(1-s+\delta)\right)}= \begin{cases}2(2 \pi)^{-s} \Gamma(s) \cos \left(\frac{\pi s}{2}\right), & \text { if } \delta=0, \\ 2 i(2 \pi)^{-s} \Gamma(s) \sin \left(\frac{\pi s}{2}\right), & \text { if } \delta=1 .\end{cases}
$$

$\Upsilon$ is called the Hankel transform of index $(\boldsymbol{\lambda}, \boldsymbol{\delta})$ of $v]^{\Uparrow}$ According to [MS3, §6], $\Upsilon$ is smooth on $\mathbb{R}^{\times}$and decays rapidly at infinity, along with all its derivatives. At the origin, $\Upsilon$ has singularities of some very particular type. Indeed, $\Upsilon(x) \in \sum_{l=1}^{n} \operatorname{sgn}(x)^{\delta_{l}}|x|^{\lambda_{l}} \mathscr{S}(\mathbb{R})$ when no two components of $\lambda$ differ by an integer, and in the nongeneric case powers of $\log |x|$ will be included.

By the Mellin inversion,

$$
\Upsilon(x)=\sum_{\delta \in \mathbb{Z} / 2 \mathbb{Z}} \frac{\operatorname{sgn}(x)^{\delta}}{4 \pi i} \int_{(\sigma)}\left(\prod_{l=1}^{n} G_{\delta_{l}+\delta}\left(s-\lambda_{l}\right)\right) \mathcal{M}_{\delta} v(1-s)|x|^{-s} d s,
$$

for $\sigma>\max \left\{\Re e \lambda_{l}\right\}$.

An alternative description of $\Upsilon$ is given by the Fourier type transform, in symbolic notion, as follows

$$
\Upsilon(x)=\frac{1}{|x|} \int_{\mathbb{R}^{\times n}} v\left(\frac{x_{1} \ldots x_{n}}{x}\right)\left(\prod_{l=1}^{n}\left(\operatorname{sgn}\left(x_{l}\right)^{\delta_{l}}\left|x_{l}\right|^{-\lambda_{l}} e\left(x_{l}\right)\right)\right) d x_{n} d x_{n-1} \ldots d x_{1} .
$$

\footnotetext{
${ }^{\mathrm{I}}$ Note that if $v$ is the $f$ in [MS4] then $|x| \Upsilon\left((-)^{n} x\right)$ is their $F(x)$.
} 
The integral converges when performed as iterated integral in the order $d x_{n} d x_{n-1} \ldots d x_{1}$, starting from $x_{n}$, then $x_{n-1}, \ldots$, and finally $x_{1}$, provided $\mathfrak{R e} \lambda_{1}>\ldots>\mathfrak{R e} \lambda_{n-1}>\mathfrak{R e} \lambda_{n}$, and it has meaning for arbitrary values of $\lambda \in \mathbb{C}^{n}$ by analytic continuation.

When applying the Voronoï summation formula for $\mathrm{GL}_{3}(\mathbb{Z})$ (currently, there is no application for rank $n \geqslant 4$ ), one is normally reduced to the asymptotic behaviour of Hankel transforms. According to [MS4], though less suggestive than $[0.8$, the expression (0.7) of Hankel transforms is more useful in applications. Indeed, all the applications of the Voronoï summation formula in analytic number theory so far are based on 0.7 with exclusive use of Stirling's asymptotic formula of the Gamma function (see [Ivi, Mil, Li2, Blo] and Appendix B]. Applications include the estimates for additively twisted sums for $\mathrm{GL}_{3}([\mathbf{M i l}])$ and for shifted convolution sums for $\mathrm{GL}_{3} \times \mathrm{GL}_{2}([\mathbf{M u n}])$, the subconvexity and non-vanishing of central values of automorphic $L$-functions for $\mathrm{GL}_{3}$ and $\mathrm{GL}_{3} \times \mathrm{GL}_{2}([\overline{\mathbf{L i 2}}, \mathbf{L i 1}, \mathbf{B l o}])$. On the other hand, there is no occurrence of the Fourier type integral transform $(0.8)$ in the literature other than Miller and Schmid's foundational work. It will however be shown in this article that the expression 0.8 should not be of only aesthetic interest.

\section{Outline of Article}

The main purpose of this article is to develop the analytic theory of fundamental Bessel functions and Bessel kernel $[$, with particular focus on their asymptotic formulae. Other subjects of this paper include analytic investigations of Hankel transforms and representation theoretic interpretations of Bessel kernels.

Enlightened by the work of Inchino and Templier [IT], we shall first study Hankel transforms over both Archimedean local fields $\mathbb{R}$ and $\mathbb{C}$. With some refinements, our treatment of Hankel transforms over $\mathbb{R}$ is essentially the same as that in [MS1]. The author however has resisted the temptation of establishing here the distribution theory on Hankel transforms over $\mathbb{C}$ from the perspective of [MS1], mainly because it would take us too far afield from the analytic theory of Bessel functions. It is very likely that this will lead to the theory of automorphic distributions on $\mathrm{GL}_{n}(\mathbb{C})$ with respect to congruence subgroups, as well as the Voronoï summation formula for cuspidal automorphic representations of $\mathrm{GL}_{n}(\mathbb{C})$. Such a formula in this generality is already covered by $[\mathbf{I T}]$, but this approach would still be of its own interest.

In Chapter 1 it will be shown that there are two expressions for Hankel transforms over either $\mathbb{R}$ or $\mathbb{C}$ and they admit integral kernels in the sense that

$$
\Upsilon(x)=\int_{\mathbb{F}^{\times}} v(y) J(x y) d y,
$$

with $\mathbb{F}=\mathbb{R}$ or $\mathbb{C}$. Thus the asymptotics of Hankel transforms may be deduced from those of their Bessel kernels. Furthermore, we shall be interested in a type of Hankel transforms over $\mathbb{R}_{+}$which is more fundamental. We now give a brief introduction of such Hankel transforms and their integral kernels.

\footnotetext{
${ }^{\mathrm{II}}$ The Bessel functions and Bessel kernels studied here are called fundamental in order to be distinguished from the Bessel functions for $\mathrm{GL}_{n}(\mathbb{F})$ arising in the Kuznetsov trace formula, with $\mathbb{F}=\mathbb{R}$ or $\mathbb{C}$. The latter should be regarded as the foundation of harmonic analysis on $\mathrm{GL}_{n}(\mathbb{F})$. Some evidences show that fundamental Bessel functions are actually the building blocks of the Bessel functions for $\mathrm{GL}_{n}(\mathbb{R})$. See [Qi1 §3.2] for the case of $\mathrm{GL}_{3}(\mathbb{F})$.

Throughout this article, we shall drop the adjective fundamental for brevity. Moreover, the usual Bessel functions will be referred to as classical Bessel functions.
} 
Given $(\lambda, \boldsymbol{S}) \in \mathbb{C}^{n} \times\{+,-\}^{n}$, with every Schwartz function $v$ on $\mathbb{R}_{+}$, which decays rapidly at both zero and infinity, we associate a function $\Upsilon$ on $\mathbb{R}_{+}$satisfying the identity

$$
\mathcal{M} \Upsilon(s)=\left(\prod_{l=1}^{n} G\left(s-\lambda_{l}, \varsigma_{l}\right)\right) \mathcal{M} v(1-s),
$$

where $\mathcal{M}$ is the usual Mellin transform over $\mathbb{R}_{+}$and $G(s, \pm)$ is the gamma factor defined by

$$
G(s, \pm)=\Gamma(s) e\left( \pm \frac{s}{4}\right) .
$$

$\Upsilon$ is called the Hankel transform of index $(\lambda, \boldsymbol{\varsigma})$ of $v$. The Mellin inversion yields the first expression of this Hankel transform,

$$
\Upsilon(x)=\frac{1}{2 \pi i} \int_{(\sigma)}\left(\prod_{l=1}^{n} G\left(s-\lambda_{l}, \varsigma_{l}\right)\right) \mathcal{M} v(1-s) x^{-s} d s, \quad \sigma>\max \left\{\mathfrak{R e} \lambda_{l}\right\} .
$$

On the other hand, under the condition $\Re e \lambda_{1}>\ldots>\Re e \lambda_{n-1}>\Re e \lambda_{n}$, the second expression is given by

$$
\Upsilon(x)=\frac{1}{x} \int_{\mathbb{R}_{+}^{n}} v\left(\frac{x_{1} \ldots x_{n}}{x}\right)\left(\prod_{l=1}^{n} x_{l}^{-\lambda_{l}} e^{\varsigma i x_{l}}\right) d x_{n} \ldots d x_{1} .
$$

We stress that these two expressions, $(0.9)$ and $(0.10)$, of Hankel transforms over $\mathbb{R}_{+}$are intimately close to those of Hankel transforms over $\mathbb{R}$, namely $(0.7)$ and $(0.8)$, respectively. We also have the kernel formula

$$
\Upsilon(x)=\int_{\mathbb{R}_{+}} v(y) J(x y ; \boldsymbol{s}, \lambda) d y,
$$

in which the kernel $J(x ; \boldsymbol{s}, \lambda)$ is called the Bessel function of index $(\boldsymbol{\varsigma}, \lambda)$.

The first expression 0.90 of the Hankel transform of index $(\lambda, \boldsymbol{s})$ yields a formula of the Bessel function $J(x ; \boldsymbol{\varsigma}, \lambda)$ as a certain Mellin-Barnes type integral involving the Gamma function (see (3.6)). Moreover, the analytic continuation of $J(x ; \boldsymbol{s}, \lambda)$ from $\mathbb{R}_{+}$ onto the Riemann surface $\mathbb{U}$, the universal cover of $\mathbb{C} \backslash\{0\}$, can be realized as a Barnes type integral via modifying the integral contour of a Mellin-Barnes type integral (see (3.8)).

As alluded to above, the asymptotics of $J(x ; \boldsymbol{s}, \lambda)$ may be obtained from applying Stirling's asymptotic formula to the Mellin-Barnes type integral (see Appendix B). This is the only known method in the literature. There are however two limitations of this method. Firstly, it is not applicable to a Barnes type integral and therefore the domain of the asymptotic expansion can not be extended from $\mathbb{R}_{+}$. Secondly, it is only applicable when $\lambda$ is regarded as fixed constant and hence the dependence on $\lambda$ of the error term can not be clarified.

The second expression 0.10$)$ of the Hankel transform of index $(\lambda, \boldsymbol{s})$ leads to a representation of the Bessel function $J(x ; \boldsymbol{\varsigma}, \lambda)$ as formal integral

$$
J_{\boldsymbol{v}}(x ; \boldsymbol{\varsigma})=\int_{\mathbb{R}_{+}^{n-1}}\left(\prod_{l=1}^{n-1} t_{l}^{\nu_{l}-1}\right) e^{i x\left(\varsigma_{n} t_{1} \ldots t_{n-1}+\sum_{l=1}^{n-1} S t_{l}^{-1}\right)} d t_{n-1} \ldots d t_{1},
$$

with $v_{l}=\lambda_{l}-\lambda_{n}$, provided that the index $\lambda$ satisfies the condition $\sum_{l=1}^{n} \lambda_{l}=0$. Accordingly, we define the complex hyperplane $\mathbb{L}^{n-1}=\left\{\lambda \in \mathbb{C}^{n}: \sum_{l=1}^{n} \lambda_{l}=0\right\}$.

The novelty of this article is an approach to Bessel functions and Bessel kernels starting from the second expression $(0.10)$ of Hankel transforms over $\mathbb{R}_{+}$. This approach is more accessible from the perspective of harmonic analysis, at least in symbolic notions. 
Once we can make sense of the symbolic notions in 0.11 , some well-developed methods from analysis and differential equations may be exploited so that we are able to understand Bessel functions and Bessel kernels to a much greater extent.

Chapter 2 is devoted to the investigations of the formal integrals $J_{\boldsymbol{v}}(x ; \boldsymbol{\varsigma})$ and the Bessel differential equations satisfied by $J(x ; \boldsymbol{s}, \lambda)$.

First of all, we must justify the formal integral $J_{v}(x ; \boldsymbol{\varsigma})$ as a representation of $J(x ; \boldsymbol{\varsigma}, \lambda)$. For this, we partition the formal integral $J_{v}(x ; \boldsymbol{\varsigma})$ according to some partition of unity on $\mathbb{R}_{+}^{n-1}$, and then repeatedly apply two kinds of partial integration operators on each resulting integral. In this way, $J_{v}(x ; \boldsymbol{s})$ can be transformed into a finite sum of absolutely convergent multiple integrals. This sum of integrals is regarded as the rigorous definition of $J_{v}(x ; \boldsymbol{S})$. Furthermore, it is shown that

$$
J(x ; \boldsymbol{\varsigma}, \lambda)=J_{v}(x ; \boldsymbol{\varsigma}),
$$

where $J_{v}(x ; \boldsymbol{s})$ on the right is now rigorously understood.

Either adapting techniques or applying results from the method of stationary phase due to Hörmander, we then study the asymptotic behaviour of each oscillatory multiple integral in the rigorous definition of $J_{v}(x ; \boldsymbol{\varsigma})$, and hence $J_{v}(x ; \boldsymbol{\varsigma})$ itself, for large argument. Even in the classical case $n=2$, our method is entirely new, as the coefficients in the asymptotic expansions are formulated in a way that is quite different from what is known in the literature (see $\$ 9.4 .3$.

When all the components of $\boldsymbol{\varsigma}$ are identically \pm , we denote $J(x ; \boldsymbol{s}, \lambda)$, respectively $J_{v}(x ; \boldsymbol{\varsigma})$, by $H^{ \pm}(x ; \lambda)$, respectively $H_{\nu}^{ \pm}(x)$, and call it an $H$-Bessel function ${ }^{m}$ This pair of $H$-Bessel functions will be of paramount significance in our treatment. It is shown that $H^{ \pm}(x ; \lambda)=H_{v}^{ \pm}(x)$ admits an analytic continuation from $\mathbb{R}_{+}$onto the half-plane $\mathbb{H}^{ \pm}=$ $\{z \in \mathbb{C} \backslash\{0\}: 0 \leqslant \pm \arg z \leqslant \pi\}$. Furthermore, we have, roughly speaking, the following asymptotic on $\mathbb{H}^{ \pm}$,

$$
H^{ \pm}(z ; \lambda) \sim n^{-\frac{1}{2}}( \pm 2 \pi i)^{\frac{n-1}{2}} e^{ \pm i n z} z^{-\frac{n-1}{2}}, \quad \text { as }|z| \rightarrow \infty .
$$

All the other Bessel functions are called K-Bessel functions and are shown to be Schwartz functions at infinity.

In the second part of Chapter 2, we discover and study the ordinary differential equation, namely Bessel equation, satisfied by the Bessel function $J(x ; \boldsymbol{\varsigma}, \lambda)$.

Given $\lambda \in \mathbb{L}^{n-1}$, there are exactly two Bessel equations

$$
\sum_{j=1}^{n} V_{n, j}(\lambda) x^{j} w^{(j)}+\left(V_{n, 0}(\lambda)-\varsigma(i n)^{n} x^{n}\right) w=0, \quad \varsigma \in\{+,-\},
$$

where $V_{n, j}(\lambda)$ is some explicitly given symmetric polynomial in $\lambda$ of degree $n-j$. We call $\varsigma$ the sign of the Bessel equation 0.13 . Then $J(x ; \boldsymbol{\varsigma}, \lambda)$ satisfies the Bessel equation of $\operatorname{sign} S_{n}(\boldsymbol{S})=\prod_{l=1}^{n} S_{l}$.

Replacing $x$ by $z$ to stand for complex variable in the Bessel equation 0.13 , the domain is extended from $\mathbb{R}_{+}$to $\mathbb{U}$. According to the theory of linear ordinary differential equations with analytic coefficients, $J(x ; \boldsymbol{\varsigma}, \lambda)$ admits an analytic continuation onto $\mathbb{U}$.

\footnotetext{
IIII a statement or a formula includes \pm or $\mp$, then it should be read with \pm and $\mp$ simultaneously replaced by either + and - or - and + .
} 
Firstly, since zero is a regular singularity, the Frobenius method may be exploited to find a solution $J_{l}(z ; \varsigma, \lambda)$ of 0.13 , for each $l=1, \ldots, n$, defined by the following series,

$$
J_{l}(z ; \varsigma, \lambda)=\sum_{m=0}^{\infty} \frac{\left(\varsigma i^{n}\right)^{m} z^{n\left(-\lambda_{l}+m\right)}}{\prod_{k=1}^{n} \Gamma\left(\lambda_{k}-\lambda_{l}+m+1\right)} .
$$

$J_{l}(z ; \varsigma, \lambda)$ will be called Bessel functions of the first kind, since they generalize the Bessel functions $J_{v}(z)$ and the modified Bessel functions $I_{v}(z)$ of the first kind.

It turns out that each $J(z ; \boldsymbol{\varsigma}, \lambda)$ may be expressed in terms of $J_{l}\left(z ; S_{n}(\boldsymbol{\varsigma}), \lambda\right)$. This leads to the following connection formula

$$
J(z ; \boldsymbol{s}, \lambda)=e\left( \pm \frac{\sum_{l \in L_{\mp}(\boldsymbol{s})} \lambda_{l}}{2}\right) H^{ \pm}\left(e^{ \pm \pi i \frac{n_{\mp}(\boldsymbol{s})}{n}} z ; \lambda\right),
$$

where $L_{ \pm}(\boldsymbol{\varsigma})=\left\{l: \varsigma_{l}= \pm\right\}$ and $n_{ \pm}(\boldsymbol{\varsigma})=\left|L_{ \pm}(\boldsymbol{s})\right|$. Thus the Bessel function $J(z ; \boldsymbol{s}, \boldsymbol{\lambda})$ is determined up to a constant by the pair of integers $\left(n_{+}(\boldsymbol{s}), n_{-}(\boldsymbol{s})\right)$, called the signature of $J(z ; \boldsymbol{\varsigma}, \lambda)$.

Secondly, infinity is an irregular singularity of rank one. Let $\xi$ be an $n$-th root of $\varsigma 1$. Then there exists a unique solution $J(z ; \lambda ; \xi)$ of the Bessel equation of sign $\varsigma$ satisfying the asymptotic

$$
J(z ; \lambda ; \xi) \sim e^{i n \xi z} z^{-\frac{n-1}{2}}, \quad \text { as }|z| \rightarrow \infty,
$$

on the sector

$$
\mathbb{S}_{\xi}=\left\{z \in \mathbb{U}:|\arg z-\arg (i \bar{\xi})|<\frac{\pi}{n}\right\},
$$

or any of its open subsector. Furthermore, the asymptotic in 0.15 may be extended onto a wider sector,

$$
\mathbb{S}_{\xi}^{\prime}=\left\{z \in \mathbb{U}:|\arg z-\arg (i \bar{\xi})|<\pi+\frac{\pi}{n}\right\} .
$$

We remark that it has been successful in attaining an optimal error estimate with respect to the index $\lambda$ in the asymptotic formula.

For a $2 n$-th root of unity $\xi, J(z ; \lambda ; \xi)$ is called a Bessel function of the second kind. We have the following formula that relates all the the Bessel functions of the second kind to either $J(z ; \lambda ; 1)$ or $J(z ; \lambda ;-1)$ upon rotating the argument by a $2 n$-th root of unity,

$$
J(z ; \lambda ; \xi)=( \pm \xi)^{\frac{n-1}{2}} J( \pm \xi z ; \lambda ; \pm 1) .
$$

The third part of Chapter 2 is on the connection formulae among Bessel functions $J(z ; \boldsymbol{\varsigma}, \lambda), J_{l}(z ; \varsigma, \lambda)$ and $J(z ; \lambda ; \xi)$. The most fundamental identity is

$$
H^{ \pm}(z ; \lambda)=n^{-\frac{1}{2}}( \pm 2 \pi i)^{\frac{n-1}{2}} J(z ; \lambda ; \pm 1) .
$$

The theory of formal integrals and the asymptotic theory of Bessel equations are both indispensable in establishing this idenity. Its proof however is simply comparing the asymptotic formulae of $H^{ \pm}(z ; \lambda)$ and $J(z ; \lambda ; \pm 1)$ in 0.12 and $(0.15)$ and using the inclusion $\mathbb{S}_{ \pm 1} \subset \mathbb{H}^{ \pm}$. We stress that the identity 0.17 can not be proven by the existing method for the asymptotic of $H^{ \pm}(x ; \lambda)$ in the literature. The reason is that Stirling's asymptotic formula can only yield an asymptotic formula of $H^{ \pm}(z ; \lambda)$ on $\mathbb{R}_{+}$, but $\mathbb{R}_{+}$does not intersect $\mathbb{S}_{ \pm 1}$ ! See Remark 12.2, B.1 for more details. With 0.17 , it follows from 0.14 and 0.16 that

$$
J(z ; \boldsymbol{\varsigma}, \lambda)=\frac{(\mp 2 \pi i)^{\frac{n-1}{2}}}{\sqrt{n}} e\left( \pm \frac{(n-1) n_{ \pm}(\boldsymbol{\varsigma})}{4 n} \mp \frac{\sum_{l \in L_{ \pm}(\boldsymbol{s})} \lambda_{l}}{2}\right) J\left(z ; \lambda ; \mp e^{\mp \pi i \frac{n_{ \pm}(\boldsymbol{s})}{n}}\right) .
$$


This actually implies the exponential decay of $K$-Bessel functions on $\mathbb{R}_{+}$. Furthermore, the identity (0.17) also yields connection formulae between the two kinds of Bessel functions, in terms of a certain Vandermonde matrix and its inverse, solving the connection formula problem for Bessel equations (see Corollary 12.5 and 12.7). Note that 0.5 and 0.6 are rank-two examples of such connection formulae.

In Chapter 3 we return to the study of Bessel kernels over real and complex numbers using results in Chapter 2 for Bessel functions.

Since a real Bessel kernel $J_{(\mu, \delta)}( \pm x)$, with $\boldsymbol{\mu} \in \mathbb{C}^{n}$ and $\delta \in(\mathbb{Z} / 2 \mathbb{Z})^{n}$, is a signed finite sum of $J\left(2 \pi x^{\frac{1}{n}} ; \boldsymbol{s}, \boldsymbol{\mu}\right)$ (see (3.48)), it is now very well understood from the investigations of $J(x ; \boldsymbol{s}, \lambda)$ in Chapter 2 .

For a complex Bessel kernel $\boldsymbol{J}_{(\boldsymbol{\mu}, \boldsymbol{m})}(z)$, with $\boldsymbol{\mu} \in \mathbb{C}^{n}$ and $\boldsymbol{m} \in \mathbb{Z}^{n}$, some extra work is required. We first prove two connection formulae between $J_{(\mu, m)}(z)$ and the two kinds of Bessel functions $J_{l}\left(z ;+, \boldsymbol{\mu} \pm \frac{1}{2} \boldsymbol{m}\right)$ and $J\left(z ; \boldsymbol{\mu} \pm \frac{1}{2} \boldsymbol{m} ; \xi\right)$, with $\xi^{n}=1$, arising from the Bessel equation of positive sign and order $n$. The connection formulae between the two kinds of Bessel functions play a crucial role in the proof. Note that in the simplest rank-one case both connection formulae reduce to the identity

$$
e(z+\bar{z})=e(z) e(\bar{z}) \text {. }
$$

Using the asymptotic formula 0.15 for $J(z ; \lambda ; \xi)$ on $\mathbb{S}_{\xi}^{\prime}$, we obtain the asymptotic

$$
J_{(\boldsymbol{\mu}, \boldsymbol{m})}\left(z^{n}\right) \sim \sum_{\xi^{n}=1} \frac{e(n(\xi z+\overline{\xi z}))}{n|z|^{n-1}[\xi z]^{|\boldsymbol{m}|}}, \quad \text { as }|z| \rightarrow \infty,
$$

where $|\boldsymbol{m}|=\sum_{l=1}^{n} m_{l}$ and $[z]=z /|z|$.

\section{Applications in Analytic Number Theory}

As mentioned earlier, along with the asymptotic formula for Bessel kernels for $\mathrm{GL}_{2}(\mathbb{R})$ or $\mathrm{GL}_{3}(\mathbb{R})$, obtained from the Stirling asymptotic formula, the Voronoï summation formula for $\mathrm{GL}_{2}$ or $\mathrm{GL}_{3}$ over $\mathbb{Q}$ has already had many applications in analytic number theory.

The very first application of the $\mathrm{GL}_{3}$ Voronoï summation formula is Miller's bound for the additive twists for $\mathrm{GL}_{3}$-automorphic forms over $\mathbb{Q}$ [Mil]. In a recent paper [Qi5], the author has generalized Miller's bound for $\mathrm{GL}_{3}$ as well as the classical bound of Wilton for $\mathrm{GL}_{2}$ to an arbitrary number field.

The author certainly hopes that the present work will have more applications in analytic number theory in the future.

\section{Formulae for Bessel Functions and Representation Theory}

In the literature of representation theory, the special functions arising in the Kuznetsov trace formula or the relative trace formula of Jacquet are also called Bessel functions.

In $\$ 18$ we shall prove a kernel formula for an infinite dimensional irreducible admissible representation of $\mathrm{GL}_{2}(\mathbb{F})$, with $\mathbb{F}=\mathbb{R}$ or $\mathbb{C}$, showing that the action of the long Weyl element on the Kirillov model is essentially a Hankel transform over $\mathbb{F}$. It follows the consensus that for $\mathrm{GL}_{2}(\mathbb{F})$ the Bessel functions occurring in the Kuznetsov trace formula should coincide with those in the Voronoï summation formula. This let us prove and generalize the Kuznetsov trace formula for $\mathrm{PSL}_{2}(\mathbb{Z}[i]) \backslash \mathrm{PSL}_{2}(\mathbb{C})$ in $[\mathbf{B M 5}]$, in the same way that $[\mathbf{C P S}]$ does for the Kuznetsov trace formula for $\mathrm{PSL}_{2}(\mathbb{Z}) \backslash \mathrm{PSL}_{2}(\mathbb{R})$ in $[\mathbf{K u z}]$. See [Qi2].

In the work of Baruch and Mao [BM2], two formulae on the Fourier transform of classical Bessel functions due to Hardy and Weber are interpreted as the Shimura-Waldspurger 
correspondence. Moreover, in the framework of the relative trace formula of Jacquet, the theory in [BM2], along with the corresponding non-Archimedean theory in [BM1], is used to produce a Waldspurger-type formula over a totally real number field [BM4]. With the motivation of generalizing their work to an arbitrary number field, the author recently proved an analogous formula for Bessel functions for $\mathrm{PGL}_{2}(\mathbb{C})$ in [Qi3, Qi4].

Another important formula for Bessel functions attached to irreducible unitary tempered representations of $\mathrm{SL}_{2}(\mathbb{F})$ is the Bessel-Plancherel formula, which is an analogue of Harish-Chandra's Plancherel formula. The formula for $\mathrm{SL}_{2}(\mathbb{R})$ is a combination of the inversion formulae of Kontorovich, Lebedev and Kuznetsov, and that for $\mathrm{SL}_{2}(\mathbb{C})$ is discovered in $[\overline{\mathbf{B M 5}}, \overline{\mathbf{L G}}]$. The formula for $\mathrm{SL}_{2}(\mathbb{R})$ is also interpreted as the Whittaker-Plancherel formula in [BM3].

In [Qi1] §3.2], the author found a formula of Bessel functions for $\mathrm{GL}_{3}(\mathbb{F})$, which are two-variable, in terms of fundamental Bessel kernels. It is formally derived from RankinSelberg $\mathrm{GL}_{3} \times \mathrm{GL}_{2}$ local functional equations and the $\mathrm{GL}_{2}$ Bessel-Plancherel formula. To be precise, the fundamental Bessel kernels occurring in the formula are for $\mathrm{GL}_{3} \times \mathrm{GL}_{2}$ and $\mathrm{GL}_{2}$, in which the involved representations of $\mathrm{GL}_{2}$ are all tempered. This gives the first instance of Bessel functions for groups of higher rank.

\section{Some Open Problems}

\section{Connections to Other Generalizations of Bessel Functions.}

Recently, the author came across a paper of Buttcane [But]. He discovered the twovariable Bessel functions in the Kuznetsov trace formula for $\mathrm{GL}_{3}(\mathbb{R})$ as power series expansions which generalize the classical Bessel functions of the first kind. It would be interesting to verify the connection of his Bessel functions for $\mathrm{GL}_{3}(\mathbb{R})$ to our fundamental Bessel kernels for $\mathrm{GL}_{3}(\mathbb{R}) \times \mathrm{GL}_{2}(\mathbb{R})$ and $\mathrm{GL}_{2}(\mathbb{R})$ via the formula in [Qi1, §3.2].

There are two more types of generalized Bessel functions, which are communicated to the author by Roman Holowinsky and the referee. The first is a class of matrix-valued analogues of classical Bessel and Whittaker functions. See for example [Ter, §1.2.2] and the refereneces therein. The second generalization was introduced by Everitt and Markett $[\mathbf{E M}]$. Their Bessel functions satisfy certain higher-order differential equations of even order. It would be interesting to find the connection between these and the work in this paper.

\section{Generalizations of Other Integral Representations of Bessel Functions.}

There are various integral representations of classical Bessel functions due to Bessel, Poisson, Hankel, Mehler, Sonine, Schläfli, Basset, Barnes and many others (see for example [Wat $]$ ). These integral representations are elegant and useful. For example, the classical derivation after Hankel of the asymptotic formula for Hankel functions makes use of his integral representation ([Wat 7.2]).

The question is, can these integral representations be generalized for our Bessel functions? Indeed, our Mellin-Barnes or Barnes type integrals and formal integrals are generalizations of the Barnes integrals and variants of the Mehler-Sonine integrals ([Wat, 6.21 $(10,11), 6.22$ (13), 6.5, 6.51]), respectively. How about other integral representations? In particular, is it admissible to generalize Hankel's integral representation and apply its generalization to the asymptotic problem along the classical line? 
Further Study on the Bessel Equations. The simplest examples of our Bessel equations of order three are

$$
x^{3} \frac{d^{3} w}{d x^{3}}+3 x^{2} \frac{d^{2} w}{d x^{2}}+\left(1-9 v^{2}\right) x \frac{d w}{d x} \mp 27 i x^{3} w=0,
$$

which have index $(v, 0,-v)$. We would be interested in the cases $v=t$ and $v=i t, t>0$. These cases are of interest in number theory because $(k-1,0,-k+1)$ and $(2 i t, 0,-2 i t)$ are the parameters of the symmetric square lifts of a holomorphic cusp form of weight $k$ and a Maaß form of eigenvalue $\frac{1}{4}+t^{2}$ for $\mathrm{SL}_{2}(\mathbb{Z})$ respectively.

While there are many problems in the theory of special functions and differential equations, we propose here the asymptotics problem of the Bessel functions for $(0.19)$ when $|v|=t$ is large. For classical Bessel functions, some basic results on this problem may be found in [Wat, Chapter 8]. Further, by methods in differential equations, the work of Olver [Olv1, Olv2] also contributes some deep results, including certain uniform asymptotic expansions which involve exponential or Airy function for the Bessel or modified Bessel functions; see also [0lv3]. It would certainly be interesting to see what special functions will replace the exponential and Airy function in our asymptotic problem for 0.19 .

Acknowledgements. The material in this article forms the first two chapters of the author's Ph.D. thesis [Qi1]. The author is grateful to his advisor, Roman Holowinsky, who brought him to the area of analytic number theory, gave him much enlightenment, guidance and encouragement. The author would like to thank James W. Cogdell, Ovidiu Costin, Stephen D. Miller, Zhilin Ye, Pengyu Yang and Cheng Zheng for valuable comments and helpful discussions. The author would like to acknowledge the inspiration from the series of papers of Stephen D. Miller and Wilfried Schmid on the Voronoï summation formula, without which this paper would never exist. The author is also indebted to the referee for several suggestions which helped improving the paper. 


\section{Notation}

- Denote $\mathbb{N}=\{0,1,2 \ldots\}$ and $\mathbb{N}_{+}=\{1,2,3, \ldots\}$.

- The group $\mathbb{Z} / 2 \mathbb{Z}$ is usually identified with the two-element set $\{0,1\}$. For $m \in \mathbb{Z}$ define $\delta(m)=m(\bmod 2)$.

- Denote $\mathbb{R}_{+}=(0, \infty), \overline{\mathbb{R}}_{+}=[0, \infty), \mathbb{R}^{\times}=\mathbb{R} \backslash\{0\}$ and $\mathbb{C}^{\times}=\mathbb{C} \backslash\{0\}$.

- Denote by $\mathbb{U} \cong \mathbb{R}_{+} \times \mathbb{R}$ the universal cover of $\mathbb{C} \backslash\{0\}$. Each element $z \in \mathbb{U}$ is denoted by $z=x e^{i \omega}=e^{\log x+i \omega}$, with $(x, \omega) \in \mathbb{R}_{+} \times \mathbb{R}$.

- Define $z^{\lambda}=e^{\lambda \log z}$ and $\bar{z}=e^{-\log z}$ for $z \in \mathbb{U}, \lambda \in \mathbb{C}$. Let $1=e^{0},-1=e^{\pi i}$ and $\pm i=e^{ \pm \frac{1}{2} \pi i}$.

- For $m \in \mathbb{Z}$ define $\delta(m) \in \mathbb{Z} / 2 \mathbb{Z}$ by $\delta(m)=m(\bmod 2)$.

- For $z \in \mathbb{C}$ let $e(z)=e^{2 \pi i z}$.

- For $s \in \mathbb{C}$ and $\alpha \in \mathbb{N}$, let $[s]_{\alpha}=\prod_{\kappa=0}^{\alpha-1}(s-\alpha)$ and $(s)_{\alpha}=\prod_{\kappa=0}^{\alpha-1}(s+\alpha)$ if $\alpha \geqslant 1$, and let $[s]_{0}=(s)_{0}=1$.

- For $\lambda=\left(\lambda_{1}, \ldots, \lambda_{n}\right) \in \mathbb{C}^{n}$ denote $|\lambda|=\sum_{l=1}^{n} \lambda_{l}$ (this notation is also used for $(\mathbb{Z} / 2 \mathbb{Z})^{n}=\{0,1\}^{n}$ and $\mathbb{Z}^{n}$ viewed as subsets of $\left.\mathbb{C}^{n}\right)$.

- Define the hyperplane $\mathbb{L}^{n-1}=\left\{\lambda \in \mathbb{C}^{n}:|\lambda|=\sum_{l=1}^{n} \lambda_{l}=0\right\}$.

- Denote by $\boldsymbol{e}^{n}$ the $n$-tuple $(1, \ldots, 1)$.

- For $l=1,2, \ldots, n$, denote $\boldsymbol{e}_{l}=(\underbrace{0, \ldots, 0,1}_{l}, 0 \ldots, 0)$ and $\boldsymbol{e}^{l}=(\underbrace{1, \ldots, 1}_{l}, 0 \ldots, 0)$.

- For $\boldsymbol{m}=\left(m_{1}, \ldots, m_{n}\right) \in \mathbb{Z}^{n}$ define $\|\boldsymbol{m}\|=\left(\left|m_{1}\right|, \ldots,\left|m_{n}\right|\right)$.

- For $\boldsymbol{s}=\left(\varsigma_{1}, \ldots, \varsigma_{n}\right) \in\{+,-\}^{n}$ denote $S_{n}(\boldsymbol{s})=|\boldsymbol{s}|=\prod_{l=1}^{n} \varsigma_{l}$.

- For $\boldsymbol{\varsigma} \in\{+,-\}^{n}$ define $L_{ \pm}(\boldsymbol{s})=\left\{l: \boldsymbol{s}_{l}= \pm\right\}$ and $n_{ \pm}(\boldsymbol{s})=\left|L_{ \pm}(\boldsymbol{s})\right|$.

- For $\boldsymbol{\varsigma} \in\{+,-\}^{n}$ and $\boldsymbol{\delta} \in(\mathbb{Z} / 2 \mathbb{Z})^{n}$ denote $\boldsymbol{\varsigma}^{\delta}=\prod_{l=1}^{n} \boldsymbol{\varsigma}_{l}^{\delta_{l}}$. 



\section{CHAPTER 1}

\section{Hankel Transforms and Bessel Kernels}

This chapter is devoted to the study of Hankel transforms over $\mathbb{R}_{+}, \mathbb{R}$ and $\mathbb{C}$ and the associated Bessel functions and Bessel kernels.

In $\$ 1$ some basic notions are introduced, such as gamma factors, Schwartz spaces, the Fourier transform and Mellin transforms. The three kinds of Mellin transforms $\mathcal{M}, \mathcal{M}_{\mathbb{R}}$ and $\mathcal{M}_{\mathbb{C}}$ are first defined over the Schwartz spaces over $\mathbb{R}_{+}, \mathbb{R}^{\times}$and $\mathbb{C}^{\times}$respectively.

In $\$ 2$ the definitions of the Mellin transforms $\mathcal{M}, \mathcal{M}_{\mathbb{R}}$ and $\mathcal{M}_{\mathbb{C}}$ are extended onto certain function spaces $\mathscr{S}_{\text {sis }}\left(\mathbb{R}_{+}\right), \mathscr{S}_{\text {sis }}\left(\mathbb{R}^{\times}\right)$and $\mathscr{S}_{\text {sis }}\left(\mathbb{C}^{\times}\right)$respectively. We shall precisely characterize their image spaces $\mathscr{M}_{\text {sis }}, \mathscr{M}_{\text {sis }}^{\mathbb{R}}$ and $\mathscr{M}_{\text {sis }}^{\mathbb{C}}$ under their corresponding Mellin transforms. In spite of their similar constructions, the analysis of the Mellin transform $\mathcal{M}_{\mathbb{C}}$ is much more elaborate than that of $\mathcal{M}_{\mathbb{R}}$ or $\mathcal{M}$.

In $\$ 3$ based on gamma factors and Mellin transforms, we shall construct Hankel transforms upon suitable subspaces of the $\mathscr{S}_{\text {sis }}$ function spaces just introduced in $\$ 2$ and show that they admit integral kernels, namely Bessel kernels, in the form of Mellin-Barnes type integrals.

In $\$ 4$ we shall compute Bessel functions and Bessel kernels in the classical cases.

In \$5 we shall first introduce the Schmid-Miller transforms in companion with the Fourier transform and then use them to establish a Fourier type integral transform expression of a Hankel transform.

In $\$ 6$ we shall introduce certain integrals, derived from the Fourier type integral transforms given in $\$ 5$, that represent Bessel functions and Bessel kernels. For Bessel functions and real Bessel kernels, these integrals are only formal and never absolutely converge. In the complex case, however, some range of index can be found where such integrals are absolutely convergent.

In Appendix A, for an arbitrary rank, we shall give a prototypical example of Bessel functions which represents their asymptotic nature.

\section{Preliminaries}

\subsection{Gamma Factors.}

1.1.1. We define the gamma factor

$$
G(s, \pm)=\Gamma(s) e\left( \pm \frac{s}{4}\right)
$$

For $(\boldsymbol{s}, \lambda)=\left(\varsigma_{1}, \ldots, \varsigma_{n}, \lambda_{1}, \ldots, \lambda_{n}\right) \in\{+,-\}^{n} \times \mathbb{C}^{n}$ let

$$
G(s ; \boldsymbol{s}, \lambda)=\prod_{l=1}^{n} G\left(s-\lambda_{l}, \varsigma_{l}\right) .
$$


1.1.2. For $\delta \in \mathbb{Z} / 2 \mathbb{Z}=\{0,1\}$, we define the gamma factor

$$
G_{\delta}(s)=i^{\delta} \pi^{\frac{1}{2}-s} \frac{\Gamma\left(\frac{1}{2}(s+\delta)\right)}{\Gamma\left(\frac{1}{2}(1-s+\delta)\right)}= \begin{cases}2(2 \pi)^{-s} \Gamma(s) \cos \left(\frac{\pi s}{2}\right), & \text { if } \delta=0, \\ 2 i(2 \pi)^{-s} \Gamma(s) \sin \left(\frac{\pi s}{2}\right), & \text { if } \delta=1 .\end{cases}
$$

Here, we have used the duplication formula and Euler's reflection formula for the Gamma function,

$$
\Gamma(1-s) \Gamma(s)=\frac{\pi}{\sin (\pi s)}, \quad \Gamma(s) \Gamma\left(s+\frac{1}{2}\right)=2^{1-2 s} \sqrt{\pi} \Gamma(2 s) .
$$

Let $(\boldsymbol{\mu}, \boldsymbol{\delta})=\left(\mu_{1}, \ldots, \mu_{n}, \delta_{1}, \ldots, \delta_{n}\right) \in \mathbb{C}^{n} \times(\mathbb{Z} / 2 \mathbb{Z})^{n}$ and define

$$
G_{(\mu, \delta)}(s)=\prod_{l=1}^{n} G_{\delta_{l}}\left(s-\mu_{l}\right) .
$$

One observes the following simple functional relation

$$
G_{(\mu, \delta)}(1-s) G_{(-\mu, \delta)}(s)=(-1)^{|\delta|} .
$$

1.1.3. For $m \in \mathbb{Z}$, we define the gamma factor

$$
G_{m}(s)=i^{|m|}(2 \pi)^{1-2 s} \frac{\Gamma\left(s+\frac{1}{2}|m|\right)}{\Gamma\left(1-s+\frac{1}{2}|m|\right)} .
$$

Let $(\boldsymbol{\mu}, \boldsymbol{m})=\left(\mu_{1}, \ldots, \mu_{n}, m_{1}, \ldots, m_{n}\right) \in \mathbb{C}^{n} \times \mathbb{Z}^{n}$ and define

$$
G_{(\mu, \boldsymbol{m})}(s)=\prod_{l=1}^{n} G_{m_{l}}\left(s-\mu_{l}\right) .
$$

We have the functional relation

$$
G_{(\mu, \boldsymbol{m})}(1-s) G_{(-\mu, \boldsymbol{m})}(s)=(-1)^{|\boldsymbol{m}|} .
$$

1.1.4. Relations between the Three Types of Gamma Factors. We first observe that

$$
G_{\delta}(s)=(2 \pi)^{-s}\left(G(s,+)+(-)^{\delta} G(s,-)\right) .
$$

Hence

$$
G_{(\mu, \delta)}(s)=\sum_{\boldsymbol{s} \in\{+,-\}^{n}} \boldsymbol{\varsigma}^{\delta}(2 \pi)^{|\boldsymbol{\mu}|-n s} G(s ; \boldsymbol{\varsigma}, \boldsymbol{\mu}), \quad \boldsymbol{\varsigma}^{\delta}=\prod_{l=1}^{n} \boldsymbol{\varsigma}_{l}^{\delta_{l}},|\boldsymbol{\mu}|=\sum_{l=1}^{n} \mu_{l} .
$$

Euler's reflection formula and certain trigonometric identities yield

$$
\begin{aligned}
i G_{m}(s) & =i^{|m|+1} 2(2 \pi)^{-2 s} \Gamma\left(s+\frac{|m|}{2}\right) \Gamma\left(s-\frac{|m|}{2}\right) \sin \left(\pi\left(s-\frac{|m|}{2}\right)\right) \\
& =G_{\delta(m)+1}\left(s-\frac{|m|}{2}\right) G_{0}\left(s+\frac{|m|}{2}\right)=G_{\delta(m)}\left(s-\frac{|m|}{2}\right) G_{1}\left(s+\frac{|m|}{2}\right),
\end{aligned}
$$

with $\delta(m)=m(\bmod 2)$. Consequently, $G_{(\mu, m)}(s)$ may be viewed as a certain $G_{(\eta, \delta)}(s)$ of doubled rank.

Lemma 1.1. Suppose that $(\boldsymbol{\mu}, \boldsymbol{m}) \in \mathbb{C}^{n} \times \mathbb{Z}^{n}$ and $(\boldsymbol{\eta}, \boldsymbol{\delta}) \in \mathbb{C}^{2 n} \times(\mathbb{Z} / 2 \mathbb{Z})^{2 n}$ are subjected to one of the following two sets of relations

$$
\begin{aligned}
& \eta_{2 l-1}=\mu_{l}+\frac{\left|m_{l}\right|}{2}, \eta_{2 l}=\mu_{l}-\frac{\left|m_{l}\right|}{2}, \delta_{2 l-1}=\delta(m)+1, \delta_{2 l}=0 ; \\
& \eta_{2 l-1}=\mu_{l}+\frac{\left|m_{l}\right|}{2}, \eta_{2 l}=\mu_{l}-\frac{\left|m_{l}\right|}{2}, \delta_{2 l-1}=\delta(m), \quad \delta_{2 l}=1 .
\end{aligned}
$$


Then $i^{n} G_{(\mu, m)}(s)=G_{(\eta, \delta)}(s)$.

1.1.5. Stirling's Asymptotic Formula. Fix $s_{0} \in \mathbb{C}$, and let $|\arg s|<\pi-\epsilon, 0<\epsilon<\pi$. We have the following asymptotic as $|s| \rightarrow \infty$

$$
\log \Gamma\left(s_{0}+s\right) \sim\left(s_{0}+s-\frac{1}{2}\right) \log s-s+\frac{1}{2} \log (2 \pi) .
$$

If one writes $s_{0}=\rho_{0}+i t_{0}$ and $s=\rho+i t, \rho \geqslant 0$, then the right hand side is equal to

$$
\begin{aligned}
& \left(\rho_{0}+\rho-\frac{1}{2}\right) \log \sqrt{t^{2}+\rho^{2}}-\left(t_{0}+t\right) \arctan \left(\frac{t}{\rho}\right)-\rho+\frac{1}{2} \log (2 \pi) \\
& +i\left(t_{0}+t\right) \log \sqrt{t^{2}+\rho^{2}}-i t+i\left(\rho_{0}+\rho-\frac{1}{2}\right) \arctan \left(\frac{t}{\rho}\right),
\end{aligned}
$$

and therefore

$$
\left|\Gamma\left(s_{0}+s\right)\right| \sim \sqrt{2 \pi}\left(t^{2}+\rho^{2}\right)^{\frac{1}{2}\left(\rho_{0}+\rho-\frac{1}{2}\right)} e^{-\left(t_{0}+t\right) \arctan (t / \rho)-\rho} .
$$

Definition 1.2. (1). For a finite closed interval $[a, b] \subset \mathbb{R}$ define the closed vertical strip $\mathbb{S}[a, b]=\{s \in \mathbb{C}: \mathfrak{R e} s \in[a, b]\}$. The open vertical strip $\mathbb{S}(a, b)$ for a finite open interval $(a, b)$ is similarly defined.

(2). For $\lambda \in \mathbb{C}$ and $r>0$, define $\mathbb{B}_{r}(\lambda)=\{s \in \mathbb{C}:|s-\lambda|<r\}$ to be the disc of radius $r$ centered at $s=\lambda$.

Lemma 1.3. We have

$$
G(s ; \boldsymbol{S}, \lambda) \ll \lambda, a, b, r(|\mathfrak{J m} s|+1)^{n\left(\mathfrak{R e} s-\frac{1}{2}\right)-\Re e}|\lambda|,
$$

for all $s \in \mathbb{S}[a, b] \backslash \bigcup_{l=1}^{n} \bigcup_{\kappa \in \mathbb{N}} \mathbb{B}_{r}\left(\lambda_{l}-\kappa\right)$, with small $r>0$,

$$
G_{(\mu, \delta)}(s) \ll \mu, a, b, r(|\mathfrak{I m} s|+1)^{n\left(\mathfrak{R e} s-\frac{1}{2}\right)-\Re \mathfrak{R}|\boldsymbol{\mu}|},
$$

for all $s \in \mathbb{S}[a, b] \backslash \bigcup_{l=1}^{n} \bigcup_{\kappa \in \mathbb{N}} \mathbb{B}_{r}\left(\mu_{l}-\delta_{l}-2 \kappa\right)$, and

$$
G_{(\mu, \boldsymbol{m})}(s) \ll \mu, a, b, r \prod_{l=1}^{n}\left(|\mathfrak{I m} s|+\left|m_{l}\right|+1\right)^{2 \mathfrak{R e} s-2 \Re \mathfrak{R} \mu_{l}-1},
$$

for all $2 s \in \mathbb{S}[a, b] \backslash \bigcup_{l=1}^{n} \bigcup_{\kappa \in \mathbb{N}} \mathbb{B}_{r}\left(2 \mu_{l}-\left|m_{l}\right|-2 \kappa\right)$.

In other words, if $\lambda$ and $\boldsymbol{\mu}$ are given, then $G(s ; \boldsymbol{\varsigma}, \lambda), G_{(\mu, \delta)}(s)$ and $G_{(\mu, m)}(s)$ are all of moderate growth with respect to $\mathfrak{I m} s$, uniformly on vertical strips (with bounded width), and moreover $G_{(\mu, m)}(s)$ is also of uniform moderate growth with respect to $\boldsymbol{m}$.

1.2. Basic Notions for $\mathbb{R}_{+}, \mathbb{R}^{\times}$and $\mathbb{C}^{\times}$. Define $\mathbb{R}_{+}=(0, \infty), \mathbb{R}^{\times}=\mathbb{R} \backslash\{0\}$ and $\mathbb{C}^{\times}=\mathbb{C} \backslash\{0\}$. We observe the isomorphisms $\mathbb{R}^{\times} \cong \mathbb{R}_{+} \times\{+,-\}\left(\cong \mathbb{R}_{+} \times \mathbb{Z} / 2 \mathbb{Z}\right)$ and $\mathbb{C}^{\times} \cong \mathbb{R}_{+} \times \mathbb{R} / 2 \pi \mathbb{Z}$, the latter being realized via the polar coordinates $z=x e^{i \phi}$.

1.2.1. Let $\mid$ denote the ordinary absolute value on either $\mathbb{R}$ or $\mathbb{C}$, and set \|\|$_{\mathbb{R}}=\mid$ | for $\mathbb{R}$ and \|\|$=\|\|_{\mathbb{C}}=||^{2}$ for $\mathbb{C}$. Let $d x$ be the Lebesgue measure on $\mathbb{R}$, and let $d^{\times} x=$ $|x|^{-1} d x$ be the standard choice of the multiplicative Haar measure on $\mathbb{R}^{\times}$. Similarly, let $d z$ be $t$ wice the ordinary Lebesgue measure on $\mathbb{C}$ and choose the standard multiplicative Haar measure $d^{\times} z=\|z\|^{-1} d z$ on $\mathbb{C}^{\times}$. Moreover, in the polar coordinates, one has $d^{\times} z=$ $2 d^{\times} x d \phi$. For $x \in \mathbb{R}^{\times}$the sign function $\operatorname{sgn}(x)$ is equal to $x /|x|$, whereas for $z \in \mathbb{C}^{\times}$we introduce the notation $[z]=z /|z|$.

Henceforth, we shall let $\mathbb{F}$ be either $\mathbb{R}$ or $\mathbb{C}$, and occasionally let $x, y$ denote elements in $\mathbb{F}$ even if $\mathbb{F}=\mathbb{C}$. 
1.2.2. For $\delta \in \mathbb{Z} / 2 \mathbb{Z}$, we define the space $C_{\delta}^{\infty}\left(\mathbb{R}^{\times}\right)$of all smooth functions $\varphi \in$ $C^{\infty}\left(\mathbb{R}^{\times}\right)$satisfying the parity condition

$$
\varphi(-x)=(-)^{\delta} \varphi(x) .
$$

Observe that a function $\varphi \in C_{\delta}^{\infty}\left(\mathbb{R}^{\times}\right)$is determined by its restriction on $\mathbb{R}_{+}$, namely, $\varphi(x)=\operatorname{sgn}(x)^{\delta} \varphi(|x|)$. Therefore,

$$
C_{\delta}^{\infty}\left(\mathbb{R}^{\times}\right)=\operatorname{sgn}(x)^{\delta} C^{\infty}\left(\mathbb{R}_{+}\right)=\left\{\operatorname{sgn}(x)^{\delta} \varphi(|x|): \varphi \in C^{\infty}\left(\mathbb{R}_{+}\right)\right\} .
$$

For a smooth function $\varphi \in C^{\infty}\left(\mathbb{R}^{\times}\right)$, we define $\varphi_{\delta} \in C^{\infty}\left(\mathbb{R}_{+}\right)$by

$$
\varphi_{\delta}(x)=\frac{1}{2}\left(\varphi(x)+(-)^{\delta} \varphi(-x)\right), \quad x \in \mathbb{R}_{+} .
$$

Clearly,

$$
\varphi(x)=\varphi_{0}(|x|)+\operatorname{sgn}(x) \varphi_{1}(|x|) .
$$

For $m \in \mathbb{Z}$, we define the space $C_{m}^{\infty}\left(\mathbb{C}^{\times}\right)$of all smooth functions $\varphi \in C^{\infty}\left(\mathbb{C}^{\times}\right)$satisfying

$$
\varphi\left(x e^{i \phi} \cdot e^{i \phi^{\prime}}\right)=e^{i m \phi^{\prime}} \varphi\left(x e^{i \phi}\right) .
$$

A function $\varphi \in C_{m}^{\infty}\left(\mathbb{C}^{\times}\right)$is determined by its restriction on $\mathbb{R}_{+}$, namely, $\varphi(z)=[z]^{m} \varphi(|z|)$, or, in the polar coordinates, $\varphi\left(x e^{i \phi}\right)=e^{i m \phi} \varphi(x)$. Therefore,

$$
C_{m}^{\infty}\left(\mathbb{R}^{\times}\right)=[z]^{m} C^{\infty}\left(\mathbb{R}_{+}\right)=\left\{[z]^{m} \varphi(|z|)=e^{i m \phi} \varphi(x): \varphi \in C^{\infty}\left(\mathbb{R}_{+}\right)\right\} .
$$

For a smooth function $\varphi \in C^{\infty}\left(\mathbb{C}^{\times}\right)$, we let $\varphi_{m} \in C^{\infty}\left(\mathbb{R}_{+}\right)$denote the $m$-th Fourier coefficient of $\varphi$ given by

$$
\varphi_{m}(x)=\frac{1}{2 \pi} \int_{0}^{2 \pi} \varphi\left(x e^{i \phi}\right) e^{-i m \phi} d \phi .
$$

One has the Fourier expansion of $\varphi$,

$$
\varphi\left(x e^{i \phi}\right)=\sum_{m \in \mathbb{Z}} \varphi_{m}(x) e^{i m \phi} .
$$

1.2.3. Subsequently, we shall encounter various subspaces of $C^{\infty}\left(\mathbb{F}^{\times}\right)$, with $\mathbb{F}=$ $\mathbb{R}, \mathbb{C}$, for instance, $\mathscr{S}(\mathbb{F}), \mathscr{S}\left(\mathbb{F}^{\times}\right), \mathscr{S}_{\text {sis }}\left(\mathbb{F}^{\times}\right), \mathscr{S}_{\text {sis }}^{(\boldsymbol{\mu}, \boldsymbol{\delta})}\left(\mathbb{R}^{\times}\right)$and $\mathscr{S}_{\text {sis }}^{(\boldsymbol{\mu}, \boldsymbol{m})}\left(\mathbb{C}^{\times}\right)$. Here, we list three central questions that will be the guidelines of our investigations of these function spaces.

For now, we let $D$ be a subspace of $C^{\infty}\left(\mathbb{F}^{\times}\right)$. For $\mathbb{F}=\mathbb{R}$ (respectively $\mathbb{F}=\mathbb{C}$ ), we shall add a superscript or subscript $\delta$ (respectively $m$ ) to the notation of $D$, say $D_{\delta}$ (respectively $D_{m}$ ), to denote the space of $\varphi \in D$ satisfying (1.17) (respectively (1.21)). In view of (1.18) (respectively 1.22$)$, there is a subspace of $C^{\infty}\left(\mathbb{R}_{+}\right)$, say $E_{\delta}$ (respectively $E_{m}$ ), such that $D_{\delta}=\operatorname{sgn}(x)^{\delta} E_{\delta}$ (respectively $D_{m}=[z]^{m} E_{m}$ ).

Firstly, we are interested in the question,

"How to characterize the space $E_{\delta}$ (respectively $\left.E_{m}\right)$ ?".

Moreover, the subspaces $D \subset C^{\infty}\left(\mathbb{F}^{\times}\right)$that we shall consider always satisfy the following two hypotheses,

- $\varphi \in D$ implies $\varphi_{\delta} \in E_{\delta}$ for $\mathbb{F}=\mathbb{R}$ (respectively, $\varphi \in D$ implies $\varphi_{m} \in E_{m}$ for $\mathbb{F}=\mathbb{C}$ ), and

- $D$ is closed under addition. 
For $\mathbb{F}=\mathbb{R}$, under these two hypotheses, it follows from 1.20 that

$$
D=D_{0} \oplus D_{1} \cong E_{0} \times E_{1}
$$

For $\mathbb{F}=\mathbb{C}$, in view of 1.24 , the map that sends $\varphi$ to the sequence $\left\{\varphi_{m}\right\}$ of its Fourier coefficients is injective. The second question arises,

"What is the image of $D$ in $\prod_{m \in \mathbb{Z}} E_{m}$ under this map?", or equivalently,

"What conditions should a sequence $\left\{\varphi_{m}\right\} \in \prod_{m \in \mathbb{Z}} E_{m}$ satisfy in order for the Fourier series defined by (1.24) giving a function $\varphi \in D$ ?”.

Finally, after introducing the Mellin transform $\mathcal{M}_{\mathbb{F}}$, we shall focus on the question,

"What is the image of $D$ under the Mellin transform $\mathcal{M}_{\mathbb{F}}$ ?".

1.3. Schwartz Spaces. We say that a function $\varphi \in C^{\infty}\left(\mathbb{R}_{+}\right)$is smooth at zero if all of its derivatives admit asymptotics as below,

$$
\varphi^{(\alpha)}(x)=\alpha ! a_{\alpha}+O_{\alpha}(x) \text { as } x \rightarrow 0 \text {, for any } \alpha \in \mathbb{N}, \text { with } a_{\alpha} \in \mathbb{C} .
$$

Remark 1.4. Consequently, one has the asymptotic expansion $\varphi(x) \sim \sum_{\kappa=0}^{\infty} a_{\kappa} x^{\kappa}$, which means that $\varphi(x)=\sum_{\kappa=0}^{A} a_{\kappa} x^{K}+O_{A}\left(x^{A+1}\right)$ as $x \rightarrow 0$ for any $A \in \mathbb{N}$. It is however not required that the series $\sum_{K=0}^{\infty} a_{K} x^{\kappa}$ be convergent for any $x \in \mathbb{R}^{\times}$.

Actually, 1.25 is equivalent to the following

$$
\varphi^{(\alpha)}(x)=\sum_{\kappa=\alpha}^{\alpha+A} a_{\kappa}[\kappa]_{\alpha} x^{\kappa-\alpha}+O_{\alpha, A}\left(x^{A+1}\right) \text { as } x \rightarrow 0, \text { for any } \alpha, A \in \mathbb{N} .
$$

Another observation is that, for a given constant $1>\rho>0,1.25$ is equivalent to the following seemingly weaker statement,

$$
\varphi^{(\alpha)}(x)=\alpha ! a_{\alpha}+O_{\alpha, \rho}\left(x^{\rho}\right) \text { as } x \rightarrow 0, \text { for any } \alpha \in \mathbb{N}, \text { with } a_{\alpha} \in \mathbb{C} .
$$

Let $C^{\infty}\left(\overline{\mathbb{R}}_{+}\right)$denote the subspace of $C^{\infty}\left(\mathbb{R}_{+}\right)$consisting of smooth functions on $\mathbb{R}_{+}$ that are also smooth at zero.

Let $\mathscr{S}\left(\overline{\mathbb{R}}_{+}\right)$denote the space of functions in $C^{\infty}\left(\overline{\mathbb{R}}_{+}\right)$that rapidly decay at infinity along with all of their derivatives. Let $\mathscr{S}(\mathbb{F})$ denote the Schwartz space on $\mathbb{F}$, with $\mathbb{F}=$ $\mathbb{R}, \mathbb{C}$.

Let $\mathscr{S}\left(\mathbb{R}_{+}\right)$denote the space of Schwartz functions on $\mathbb{R}_{+}$, that is, smooth functions on $\mathbb{R}_{+}$whose derivatives rapidly decay at both zero and infinity. Similarly, we denote by $\mathscr{S}\left(\mathbb{F}^{\times}\right)$the space of Schwartz functions on $\mathbb{F}^{\times}$.

The following lemma provides criteria for characterizing functions in these Schwartz spaces, especially functions in $\mathscr{S}(\mathbb{C})$ or $\mathscr{S}\left(\mathbb{C}^{\times}\right)$in the polar coordinates. Its proof is left as an easy excise in analysis for the reader.

LEMMA 1.5. Let notations be as above.

(1.1). Let $\varphi \in C^{\infty}\left(\overline{\mathbb{R}}_{+}\right)$satisfy the asymptotics $[1.25)$. Then $\varphi \in \mathscr{S}\left(\overline{\mathbb{R}}_{+}\right)$if and only if $\varphi$ also satisfies

$$
x^{\alpha+\beta} \varphi^{(\alpha)}(x) \ll_{\alpha, \beta} 1 \text { for all } \alpha, \beta \in \mathbb{N} .
$$

(1.2). A smooth function $\varphi$ on $\mathbb{R}_{+}$belongs to $\mathscr{S}\left(\mathbb{R}_{+}\right)$if and only if $\varphi$ satisfies 1.28 with $\beta \in \mathbb{N}$ replaced by $\beta \in \mathbb{Z}$.

Let $\varphi \in \mathscr{S}\left(\overline{\mathbb{R}}_{+}\right)$and $a_{\alpha}$ be as in $(1.25)$. Then $\varphi \in \mathscr{S}\left(\mathbb{R}_{+}\right)$if and only if $a_{\alpha}=0$ for all $\alpha \in \mathbb{N}$.

(2.1). A smooth function $\varphi$ on $\mathbb{R}^{\times}$extends to a function in $\mathscr{S}(\mathbb{R})$ if and only if

- $\varphi$ satisfies 1.28 with $x^{\alpha+\beta}$ replaced by $|x|^{\alpha+\beta}$, and 
- all the derivatives of $\varphi$ admit asymptotics

$$
\varphi^{(\alpha)}(x)=\alpha ! a_{\alpha}+O_{\alpha}(|x|) \text { as } x \rightarrow 0, \text { for any } \alpha \in \mathbb{N} \text {, with } a_{\alpha} \in \mathbb{C} .
$$

(2.2). Let $\varphi$ be a smooth function on $\mathbb{R}^{\times}$. Then $\varphi \in \mathscr{S}\left(\mathbb{R}^{\times}\right)$if and only if $\varphi$ satisfies (1.28) with $x^{\alpha+\beta}$ replaced by $|x|^{\alpha+\beta}$ and $\beta \in \mathbb{N}$ by $\beta \in \mathbb{Z}$.

Suppose $\varphi \in \mathscr{S}(\mathbb{R})$, then $\varphi \in \mathscr{S}\left(\mathbb{R}^{\times}\right)$if and only if $\varphi^{(\alpha)}(0)=0$ for all $\alpha \in \mathbb{N}$, or equivalently, $a_{\alpha}=0$ for all $\alpha \in \mathbb{N}$, with $a_{\alpha}$ given in (1.29).

(3.1). Write $\partial_{x}=\partial / \partial x$ and $\partial_{\phi}=\partial / \partial \phi$. In the polar coordinates, a smooth function $\varphi\left(x e^{i \phi}\right) \in C^{\infty}\left(\mathbb{C}^{\times}\right)$extends to a function in $\mathscr{S}(\mathbb{C})$ if and only if

- $\varphi\left(x e^{i \phi}\right)$ satisfies

$$
x^{\alpha+\beta} \partial_{x}^{\alpha} \partial_{\phi}^{\gamma} \varphi\left(x e^{i \phi}\right) \ll_{\alpha, \beta, \gamma} 1 \text { for all } \alpha, \beta, \gamma \in \mathbb{N},
$$

- all the partial derivatives of $\varphi$ admit asymptotics

$$
x^{\alpha} \partial_{x}^{\alpha} \partial_{\phi}^{\beta} \varphi\left(x e^{i \phi}\right)=\sum_{|m| \leqslant \alpha+\beta} \sum_{\substack{|m| \leqslant \kappa \leqslant \alpha+\beta \\ \kappa \equiv m(\bmod 2)}} a_{m, \kappa}[\kappa]_{\alpha}(i m)^{\beta} x^{\kappa} e^{i m \phi}+O_{\alpha, \beta}\left(x^{\alpha+\beta+1}\right)
$$

as $x \rightarrow 0$, for any $\alpha, \beta \in \mathbb{N}$, with $a_{m, \kappa} \in \mathbb{C}$ for $\kappa \geqslant|m|$ and $\kappa \equiv m(\bmod 2)$.

Let $\varphi \in \mathscr{S}(\mathbb{C})$ and $\varphi_{m}$ be the m-th Fourier coefficient of $\varphi$ given by [1.23), then it follows from (1.30, 1.31) that

- $\varphi_{m}$ satisfies

$$
x^{\alpha+\beta} \varphi_{m}^{(\alpha)}(x) \ll_{\alpha, \beta, A}(|m|+1)^{-A} \text { for all } \alpha, \beta, A \in \mathbb{N},
$$

- all the derivatives of $\varphi_{m}$ admit asymptotics

$$
\varphi_{m}^{(\alpha)}(x)=\sum_{\kappa=\alpha}^{\alpha+A} a_{m, \kappa}[\kappa]_{\alpha} x^{\kappa-\alpha}+O_{\alpha, A}\left((|m|+1)^{-A} x^{A+1}\right)
$$

as $x \rightarrow 0$, for any given $\alpha, A \in \mathbb{N}$, with $a_{m, \kappa} \in \mathbb{C}$ satisfying $a_{m, \kappa}=0$ if either $\kappa<|m|$ or $\kappa \not \equiv m(\bmod 2)$.

Observe that (1.33) is equivalent to the following two conditions,

$\varphi_{m}^{(\alpha)}(x)=\alpha ! a_{m, \alpha}+O_{\alpha}(x)$ as $x \rightarrow 0$, for any $\alpha \geqslant|m|$, with $a_{m, \alpha} \in \mathbb{C}$ satisfying $a_{m, \alpha}=0$ if $\alpha \neq \equiv m(\bmod 2)$,

for any given $\alpha, A \in \mathbb{N}, \varphi_{m}^{(\alpha)}(x)=O_{\alpha, A}\left((|m|+1)^{-A} x^{A+1}\right)$ as $x \rightarrow 0$, if $|m|>$ $\alpha+A$.

In particular, $\varphi_{m} \in \mathscr{S}\left(\overline{\mathbb{R}}_{+}\right)$.

Conversely, if a sequence $\left\{\varphi_{m}\right\}$ of functions in $C^{\infty}\left(\mathbb{R}_{+}\right)$satisfies (1.32), (1.34) and (1.35), then the Fourier series defined by $\left\{\varphi_{m}\right\}$, that is, the right hand side of (1.24), is a Schwartz function on $\mathbb{C}$.

(3.2). In the polar coordinates, a smooth function $\varphi\left(x e^{i \phi}\right) \in C^{\infty}\left(\mathbb{C}^{\times}\right)$is a Schwartz function on $\mathbb{C}^{\times}$if and only if $\varphi$ satisfies $(1.30)$ with $\beta \in \mathbb{N}$ replaced by $\beta \in \mathbb{Z}$.

Let $\varphi \in \mathscr{S}\left(\mathbb{C}^{\times}\right)$and $\varphi_{m}$ be the $m$-th Fourier coefficient of $\varphi$, then it is necessary that $\varphi_{m}$ satisfies $\left(1.32\right.$ with $\beta \in \mathbb{N}$ replaced by $\beta \in \mathbb{Z}$. In particular, $\varphi_{m} \in \mathscr{S}\left(\mathbb{R}_{+}\right)$.

Conversely, if a sequence $\left\{\varphi_{m}\right\}$ of functions in $C^{\infty}\left(\mathbb{R}_{+}\right)$satisfies the condition 1.32 with $\beta \in \mathbb{N}$ replaced by $\beta \in \mathbb{Z}$, then the Fourier series defined by $\left\{\varphi_{m}\right\}$ gives rise to a Schwartz function on $\mathbb{C}^{\times}$.

Let $\varphi \in \mathscr{S}(\mathbb{C})$ and $a_{m, \kappa}$ be given in 1.31, (1.33) or (1.34). $\varphi \in \mathscr{S}\left(\mathbb{C}^{\times}\right)$if and only if $a_{m, \kappa}=0$ for all $m \in \mathbb{Z}, \kappa \in \mathbb{N}$. 
1.3.1. Some Subspaces of $\mathscr{S}\left(\overline{\mathbb{R}}_{+}\right)$. In the following, we introduce several subspaces of $\mathscr{S}\left(\overline{\mathbb{R}}_{+}\right)$which are closely related to $\mathscr{S}(\mathbb{R})$ and $\mathscr{S}(\mathbb{C})$.

We first define for $\delta \in \mathbb{Z} / 2 \mathbb{Z}$ the subspace $C_{\delta}^{\infty}\left(\overline{\mathbb{R}}_{+}\right) \subset C^{\infty}\left(\overline{\mathbb{R}}_{+}\right)$of functions with an asymptotic expansion of the form $\sum_{\kappa=0}^{\infty} a_{\kappa} x^{\delta+2 \kappa}$ at zero.

Remark 1.6. A question arises, "whether $C^{\infty}\left(\overline{\mathbb{R}}_{+}\right)=C_{0}^{\infty}\left(\overline{\mathbb{R}}_{+}\right)+C_{1}^{\infty}\left(\overline{\mathbb{R}}_{+}\right)$?".

The answer is affirmative.

To see this, we define the space $C_{\delta}^{\infty}(\mathbb{R})$ of smooth functions $\varphi$ on $\mathbb{R}$ satisfying (1.17). One has $\operatorname{sgn}(x)^{\delta} \varphi(|x|) \in C_{\delta}^{\infty}(\mathbb{R})$ if $\varphi \in C_{\delta}^{\infty}\left(\overline{\mathbb{R}}_{+}\right)$, and conversely, $\left.\varphi\right|_{\mathbb{R}_{+}} \in C_{\delta}^{\infty}\left(\overline{\mathbb{R}_{+}}\right)$if $\varphi \in C_{\delta}^{\infty}(\mathbb{R})$. Thus, with the simple observation $C^{\infty}(\mathbb{R})=C_{0}^{\infty}(\mathbb{R}) \oplus C_{1}^{\infty}(\mathbb{R})$, one sees that $C_{0}^{\infty}\left(\overline{\mathbb{R}}_{+}\right)+C_{1}^{\infty}\left(\overline{\mathbb{R}}_{+}\right)$is the subspace of $C^{\infty}\left(\overline{\mathbb{R}}_{+}\right)$consisting of functions on $\mathbb{R}_{+}$that admit a smooth extension onto $\mathbb{R}$.

On the other hand, the Borel theorem ([Nar, 1.5.4]), which is a special case of the Whitney extension theorem ([Nar $1.5 .5,1.5 .6])$, states that for any sequence $\left\{a_{\alpha}\right\}$ of constants there exists a smooth function $\varphi \in C^{\infty}(\mathbb{R})$ such that $\varphi^{(\alpha)}(0)=\alpha ! a_{\alpha}$. Clearly, this theorem of Borel implies our assertion above.

In $\$ 2.1 .3$ we shall give an alternative proof of this using the Mellin transform. See Remark 2.5 .

We define $\mathscr{S}_{\delta}\left(\overline{\mathbb{R}}_{+}\right)=\mathscr{S}\left(\overline{\mathbb{R}}_{+}\right) \cap C_{\delta}^{\infty}\left(\overline{\mathbb{R}}_{+}\right)$. The following identity is obvious

$$
\mathscr{S}_{\delta}\left(\overline{\mathbb{R}}_{+}\right)=x^{\delta} \mathscr{S}_{0}\left(\overline{\mathbb{R}}_{+}\right) .
$$

In view of Lemma $1.5(1.2)$, we have $\mathscr{S}_{0}\left(\overline{\mathbb{R}}_{+}\right) \cap \mathscr{S}_{1}\left(\overline{\mathbb{R}}_{+}\right)=\mathscr{S}\left(\mathbb{R}_{+}\right)$.

If we let $\mathscr{S}_{\delta}(\mathbb{R})$ be the space of functions $\varphi \in \mathscr{S}(\mathbb{R})$ satisfying (1.17), then

$$
\mathscr{S}_{\delta}(\mathbb{R})=\operatorname{sgn}(x)^{\delta} \mathscr{S}_{\delta}\left(\overline{\mathbb{R}}_{+}\right)=\left\{\operatorname{sgn}(x)^{\delta} \varphi(|x|): \varphi \in \mathscr{S}_{\delta}\left(\overline{\mathbb{R}}_{+}\right)\right\} .
$$

Clearly, $\mathscr{S}(\mathbb{R})=\mathscr{S}_{0}(\mathbb{R}) \oplus \mathscr{S}_{1}(\mathbb{R})$.

We define the subspace $\mathscr{S}_{m}\left(\overline{\mathbb{R}}_{+}\right) \subset \mathscr{S}_{\delta(m)}\left(\overline{\mathbb{R}}_{+}\right)$, with $\delta(m)=m(\bmod 2)$, of functions with an asymptotic expansion of the form $\sum_{\kappa=0}^{\infty} a_{\kappa} x^{|m|+2 \kappa}$ at zero. We have

$$
\mathscr{S}_{m}\left(\overline{\mathbb{R}}_{+}\right)=x^{|m|} \mathscr{S}_{0}\left(\overline{\mathbb{R}}_{+}\right) .
$$

If we define $\mathscr{S}_{m}(\mathbb{C})$ to be the space of $\varphi \in \mathscr{S}(\mathbb{C})$ satisfying (1.21), then

$$
\mathscr{S}_{m}(\mathbb{C})=[z]^{m} \mathscr{S}_{m}\left(\overline{\mathbb{R}}_{+}\right)=\left\{[z]^{m} \varphi(|z|)=e^{i m \phi} \varphi(x): \varphi \in \mathscr{S}_{m}\left(\overline{\mathbb{R}}_{+}\right)\right\} .
$$

The last two paragraphs in Lemma 1.5 (3.1) can be recapitulated as below

$$
\mathscr{S}(\mathbb{C}) \cong\left\{\left\{\varphi_{m}\right\} \in \prod_{m \in \mathbb{Z}} \mathscr{S}_{m}\left(\overline{\mathbb{R}}_{+}\right): \varphi_{m} \text { satisfies 1.32 1.34, 1.35) }\right\} \rightarrow \mathscr{S}_{m}\left(\overline{\mathbb{R}}_{+}\right),
$$

where the first map sends $\varphi \in \mathscr{S}(\mathbb{C})$ to the sequence $\left\{\varphi_{m}\right\}$ of its Fourier coefficients, and the second is the $m$-th projection. According to Lemma 1.5 (3.1), the first map is an isomorphism, and the second projection is surjective.

1.3.2. $\mathscr{S}_{\delta}\left(\mathbb{R}^{\times}\right)$and $\mathscr{S}_{m}\left(\mathbb{C}^{\times}\right)$. Let $\delta \in \mathbb{Z} / 2 \mathbb{Z}$ and $m \in \mathbb{Z}$. We define $\mathscr{S}_{\delta}\left(\mathbb{R}^{\times}\right)=$ $\mathscr{S}\left(\mathbb{R}^{\times}\right) \cap \mathscr{S}_{\delta}(\mathbb{R})$ and $\mathscr{S}_{m}\left(\mathbb{C}^{\times}\right)=\mathscr{S}\left(\mathbb{C}^{\times}\right) \cap \mathscr{S}_{m}(\mathbb{C})$. Clearly, $\mathscr{S}_{\delta}\left(\mathbb{R}^{\times}\right)=\operatorname{sgn}(x)^{\delta} \mathscr{S}\left(\mathbb{R}_{+}\right)$ and $\mathscr{S}_{m}\left(\mathbb{C}^{\times}\right)=[z]^{m} \mathscr{S}\left(\mathbb{R}_{+}\right)$. 
1.4. The Fourier Transform. According to the local theory in Tate's thesis for an Archimedean local field $\mathbb{F}$, the Fourier transform $\hat{\varphi}=\mathcal{F} \varphi$ of a Schwartz function $\varphi \in \mathscr{S}(\mathbb{F})$ is defined by

$$
\widehat{\varphi}(y)=\int_{\mathbb{F}} \varphi(x) e(-\Lambda(x y)) d x
$$

with

$$
\Lambda(x)= \begin{cases}x, & \text { if } \mathbb{F}=\mathbb{R} \\ \operatorname{Tr}(x)=x+\bar{x}, & \text { if } \mathbb{F}=\mathbb{C} .\end{cases}
$$

The Schwartz space $\mathscr{S}(\mathbb{F})$ is invariant under the Fourier transform. Moreover, with our choice of measure in $\$ 1.2$, the following inversion formula holds

$$
\widehat{\hat{\varphi}}(x)=\varphi(-x), \quad x \in \mathbb{F} .
$$

1.5. The Mellin Transforms $\mathcal{M}, \mathcal{M}_{\delta}$ and $\mathcal{M}_{m}$. Corresponding to $\mathbb{R}_{+}, \mathbb{R}^{\times}$and $\mathbb{C}^{\times}$, there are three kinds of Mellin transforms $\mathcal{M}, \mathcal{M}_{\delta}$ and $\mathcal{M}_{m}$.

Definition 1.7 (Mellin transforms).

(1). The Mellin transform $\mathcal{N} \varphi$ of a Schwartz function $\varphi \in \mathscr{S}\left(\mathbb{R}_{+}\right)$is given by

$$
\mathcal{M} \varphi(s)=\int_{\mathbb{R}_{+}} \varphi(x) x^{s} d^{\times} x .
$$

(2). For $\delta \in \mathbb{Z} / 2 \mathbb{Z}$, the (signed) Mellin transform $\mathcal{M}_{\delta} \varphi$ with order $\delta$ of a Schwartz function $\varphi \in \mathscr{S}\left(\mathbb{R}^{\times}\right)$is defined by

$$
\mathcal{M}_{\delta} \varphi(s)=\int_{\mathbb{R}^{\times}} \varphi(x) \operatorname{sgn}(x)^{\delta}|x|^{s} d^{\times} x .
$$

Moreover, define $\mathcal{M}_{\mathbb{R}}=\left(\mathcal{M}_{0}, \mathcal{M}_{1}\right)$.

(3). For $m \in \mathbb{Z}$, the Mellin transform $\mathcal{M}_{m} \varphi$ with order $m$ of a Schwartz function $\varphi \in \mathscr{S}\left(\mathbb{C}^{\times}\right)$is defined by

$$
\mathcal{M}_{m} \varphi(s)=\int_{\mathbb{C}^{\times}} \varphi(z)[z]^{m}\|z\|^{\frac{1}{2} s} d^{\times} z=2 \int_{0}^{\infty} \int_{0}^{2 \pi} \varphi\left(x e^{i \phi}\right) e^{i m \phi} d \phi \cdot x^{s} d^{\times} x .
$$

Moreover, define $\mathcal{M}_{\mathbb{C}}=\prod_{m \in \mathbb{Z}} \mathcal{M}_{-m}$.

Observation 1.8. For $\varphi \in \mathscr{S}\left(\mathbb{R}^{\times}\right)$, we have

$$
\mathcal{M}_{\delta} \varphi(s)=2 \mathcal{M} \varphi_{\delta}(s), \quad \delta \in \mathbb{Z} / 2 \mathbb{Z} .
$$

Similarly, for $\varphi \in \mathscr{S}\left(\mathbb{C}^{\times}\right)$, we have

$$
\mathcal{M}_{-m} \varphi(s)=4 \pi \mathcal{N} \varphi_{m}(s), \quad m \in \mathbb{Z} .
$$

The relations (1.42) and (1.43) reflect the identities $\mathbb{R}^{\times} \cong \mathbb{R}_{+} \times\{+,-\}$and $\mathbb{C}^{\times} \cong \mathbb{R}_{+} \times$ $\mathbb{R} / 2 \pi \mathbb{Z}$ respectively.

Lemma 1.9 (Mellin inversions). Let $\sigma$ be real. Denote by $(\sigma)$ the vertical line from $\sigma-i \infty$ to $\sigma+i \infty$.

(1). For $\varphi \in \mathscr{S}\left(\mathbb{R}_{+}\right)$, we have

$$
\varphi(x)=\frac{1}{2 \pi i} \int_{(\sigma)} \mathcal{M} \varphi(s) x^{-s} d s .
$$


(2). For $\varphi \in \mathscr{S}\left(\mathbb{R}^{\times}\right)$, we have

$$
\varphi(x)=\frac{1}{4 \pi i} \sum_{\delta \in \mathbb{Z} / 2 \mathbb{Z}} \operatorname{sgn}(x)^{\delta} \int_{(\sigma)} \mathcal{M}_{\delta} \varphi(s)|x|^{-s} d s .
$$

(3). For $\varphi \in \mathscr{S}\left(\mathbb{C}^{\times}\right)$, we have

$$
\varphi(z)=\frac{1}{8 \pi^{2} i} \sum_{m \in \mathbb{Z}}[z]^{-m} \int_{(\sigma)} \mathcal{M}_{m} \varphi(s)\|z\|^{-\frac{1}{2} s} d s,
$$

or, in the polar coordinates,

$$
\varphi\left(x e^{i \phi}\right)=\frac{1}{8 \pi^{2} i} \sum_{m \in \mathbb{Z}} e^{-i m \phi} \int_{(\sigma)} \mathcal{M}_{m} \varphi(s) x^{-s} d s .
$$

DeFINITION 1.10.

(1). Let $\mathscr{H}_{\text {rd }}$ denote the space of all entire functions $H(s)$ on the complex plane that rapidly decay along vertical lines, uniformly on vertical strips.

(2). Define $\mathscr{H}_{\text {rd }}^{\mathbb{R}}=\mathscr{H}_{\text {rd }} \times \mathscr{H}_{\text {rd }}$.

(3). Let $\mathscr{H}_{\mathrm{rd}}^{\mathbb{C}}$ be the subset of $\prod_{\mathbb{Z}} \mathscr{H}_{\text {rd }}$ consisting of sequences $\left\{H_{m}(s)\right\}$ of entire functions in $\mathscr{H}_{\text {rd }}$ satisfying the following condition,

$$
\begin{aligned}
& \text { for any given } \alpha, A \in \mathbb{N} \text { and vertical strip } \mathbb{S}[a, b] \\
& H_{m}(s) \ll_{\alpha, A, a, b}(|m|+1)^{-A}(|\mathfrak{I} \mathrm{m} s|+1)^{-\alpha} \text { for all } s \in \mathbb{S}[a, b] .
\end{aligned}
$$

Corollary 1.11.

(1). The Mellin transform $\mathcal{M}$ and its inversion establish an isomorphism between $\mathscr{S}\left(\mathbb{R}_{+}\right)$and $\mathscr{H}_{\mathrm{rd}}$.

(2). For each $\delta \in \mathbb{Z} / 2 \mathbb{Z}, \mathcal{M}_{\delta}$ establishes an isomorphism between $\mathscr{S}_{\delta}\left(\mathbb{R}^{\times}\right)$and $\mathscr{H}_{\mathrm{rd}}$. Hence, $\mathcal{M}_{\mathbb{R}}$ establishes an isomorphism between $\mathscr{S}\left(\mathbb{R}^{\times}\right)$and $\mathscr{H}_{\mathrm{rd}}^{\mathbb{R}}$.

(3). For each $m \in \mathbb{Z}, \mathcal{M}_{-m}$ establishes an isomorphism between $\mathscr{S}_{m}\left(\mathbb{C}^{\times}\right)$and $\mathscr{H}_{\mathrm{rd}}$. Moreover, $\mathcal{M}_{\mathbb{C}}$ establishes an isomorphism between $\mathscr{S}\left(\mathbb{C}^{\times}\right)$and $\mathscr{H}_{\mathrm{rd}}^{\mathbb{C}}$.

Proof. (1) is a well-known consequence of Lemma 1.9 (1), whereas (2) directly follows from (1) and Lemma 1.9 (2). As for (3), in addition to (1) and Lemma 1.9 (3), Lemma 1.5 (3.2) is also required for the rapid decay in $m$.

Q.E.D.

\section{The Function Spaces $\mathscr{S}_{\text {sis }}\left(\mathbb{R}_{+}\right), \mathscr{S}_{\text {sis }}\left(\mathbb{R}^{\times}\right)$and $\mathscr{S}_{\text {sis }}\left(\mathbb{C}^{\times}\right)$}

The goal of this section is to extend the definitions of the Mellin transforms $\mathcal{M}, \mathcal{M}_{\mathbb{F}}$ and generalize the settings in $\$ 1.5$ to the function spaces $\mathscr{S}_{\text {sis }}\left(\mathbb{R}_{+}\right), \mathscr{M}_{\text {sis }}, \mathscr{S}_{\text {sis }}\left(\mathbb{F}^{\times}\right)$and $\mathscr{M}_{\text {sis }}^{\mathbb{F}}$. These spaces are much more sophisticated than $\mathscr{S}\left(\mathbb{R}_{+}\right), \mathscr{H}_{\mathrm{rd}}, \mathscr{S}\left(\mathbb{F}^{\times}\right)$and $\mathscr{H}_{\mathrm{rd}}^{\mathbb{F}}$ but most suitable for investigating Hankel transforms over $\mathbb{R}_{+}$and $\mathbb{F}^{\times}$.

We shall first construct the function spaces $\mathscr{S}_{\text {sis }}\left(\mathbb{R}_{+}\right), \mathscr{M}_{\text {sis }}$ and establish an isomorphism between them using the Mellin transform $\mathcal{M}$. Based on these, we shall then turn to the spaces $\mathscr{S}_{\text {sis }}\left(\mathbb{F}^{\times}\right), \mathscr{M}_{\text {sis }}^{\mathbb{F}}$ and the Mellin transform $\mathcal{M}_{\mathbb{F}}$. The case $\mathbb{F}=\mathbb{R}$ has been worked out in [MS3, §6]. Since $\mathbb{R}^{\times} \cong \mathbb{R}_{+} \times\{+,-\}$is simply two copies of $\mathbb{R}_{+}$, the properties of $\mathscr{S}_{\text {sis }}\left(\mathbb{R}^{\times}\right)$and $\mathscr{M}_{\text {sis }}^{\mathbb{R}}$ are in substance the same as those of $\mathscr{S}_{\text {sis }}\left(\mathbb{R}_{+}\right)$and $\mathscr{M}_{\text {sis. }}$ In the case $\mathbb{F}=\mathbb{C}, \mathscr{S}_{\text {sis }}\left(\mathbb{C}^{\times}\right)$and $\mathscr{M}_{\text {sis }}^{\mathbb{C}}$ can be constructed in a parallel way. The study on $\mathbb{C}^{\times}$ is however much more elaborate, since $\mathbb{C}^{\times} \cong \mathbb{R}_{+} \times \mathbb{R} / 2 \pi \mathbb{Z}$ and the analysis on the circle $\mathbb{R} / 2 \pi \mathbb{Z}$ is also taken into account. 
2.1. The Spaces $\mathscr{S}_{\text {sis }}\left(\mathbb{R}_{+}\right)$and $\mathscr{M}_{\text {sis }} \cdot \mathbb{1}$

2.1.1. The Spaces $x^{-\lambda}(\log x)^{j} \mathscr{S}\left(\overline{\mathbb{R}}_{+}\right)$and $\mathscr{M}_{\text {sis }}^{\lambda, j}$. Let $\lambda \in \mathbb{C}$ and $j \in \mathbb{N}$.

We define

$$
x^{-\lambda}(\log x)^{j} \mathscr{S}\left(\overline{\mathbb{R}}_{+}\right)=\left\{x^{-\lambda}(\log x)^{j} \varphi(x): \varphi \in \mathscr{S}\left(\overline{\mathbb{R}}_{+}\right)\right\} .
$$

We say that a meromorphic function $H(s)$ has a pole of pure order $j+1$ at $s=\lambda$ if the principal part of $H(s)$ at $s=\lambda$ is $a(s-\lambda)^{-j-1}$ for some constant $a \in \mathbb{C}$. Of course, $H(s)$ does not have a genuine pole at $s=\lambda$ if $a=0$. We define the space $\mathscr{M}_{\text {sis }}^{\lambda, j}$ of all meromorphic functions $H(s)$ on the complex plane such that

- the only possible singularities of $H(s)$ are poles of pure order $j+1$ at the points in $\lambda-\mathbb{N}=\{\lambda-\kappa: \kappa \in \mathbb{N}\}$, and

- $H(s)$ decays rapidly along vertical lines, uniformly on vertical strips, that is, for any given $\alpha \in \mathbb{N}$, vertical strip $\mathbb{S}[a, b]$ and $r>0$,

$$
H(s) \ll \lambda, j, \alpha, a, b, r(|\mathfrak{I m} s|+1)^{-\alpha} \text { for all } s \in \mathbb{S}[a, b] \backslash \bigcup_{\kappa \in \mathbb{N}} \mathbb{B}_{r}(\lambda-\kappa) .
$$

The constructions of the Mellin transform $\mathcal{M}$ and its inversion 1.39, 1.44) identically extend from $\mathscr{S}\left(\mathbb{R}_{+}\right)$onto $\mathscr{S}_{\text {sis }}^{\lambda, j}\left(\mathbb{R}_{+}\right)$, except that the conditions $\mathfrak{R e} s>\mathfrak{R e} \lambda$ and $\sigma>\mathfrak{R e} \lambda$ are required to guarantee convergence.

Lemma 2.1. Let $\lambda \in \mathbb{C}$ and $j \in \mathbb{N}$. The Mellin transform $\mathcal{M}$ and its inversion establish an isomorphism of between $x^{-\lambda}(\log x)^{j} \mathscr{S}\left(\overline{\mathbb{R}}_{+}\right)$and $\mathscr{M}_{\text {sis }}^{\lambda, j}$.

This lemma is essentially [MS3, Lemma 6.13, Corollary 6.17]. Nevertheless, we shall include its proof as the reference for the constructions of $\mathscr{N}_{\text {sis }}^{\mathbb{C}, \lambda, j}$ and $\mathscr{M}_{\text {sis }}^{\mathbb{C}}$ in $\$ 2.3 .2$ as well as the proof of Lemma 2.8 .

Proof. Let $v(x)=x^{-\lambda}(\log x)^{j} \varphi(x)$ for some $\varphi \in \mathscr{S}\left(\overline{\mathbb{R}}_{+}\right)$. Suppose that the derivatives of $\varphi$ satisfy (1.26) and (1.28), that is, asymptotic expansions at zero and the Schwartz condition at infinity.

Claim 1. Let

$$
H(s)=\mathcal{M} v(s)=\int_{0}^{\infty} v(s) x^{s-1} d x, \quad \mathfrak{R e} s>\mathfrak{R e} \lambda .
$$

Then $H$ admits a meromorphic continuation onto the whole complex plane. The only singularities of $H$ are poles of pure order $j+1$ at the points in $\lambda-\mathbb{N}$. More precisely, $H(s)$ has a pole at $s=\lambda-\kappa$ of principal part $(-)^{j} j ! a_{\kappa}(s-\lambda+\kappa)^{-j-1}$. Moreover, $H$ decays rapidly along vertical lines, uniformly on vertical strips. To be concrete, we have

$$
\begin{aligned}
& \text { for any given } \alpha, A \in \mathbb{N}, b \geqslant a>\mathfrak{R} \lambda \lambda-\alpha-A-1 \text { and } r>0, \\
& H(s) \ll \lambda, j, \alpha, A, a, b, r(|\mathfrak{I m} s|+1)^{-\alpha} \text { for all } s \in \mathbb{S}[a, b] \backslash \bigcup_{\kappa=0}^{\alpha+A} \mathbb{B}_{r}(\lambda-\kappa) .
\end{aligned}
$$

We remark that 2.1) and 2.2) are equivalent.

Proof of Claim 1. In view of $\mathcal{M}\left(x^{-\lambda}(\log x)^{j} \varphi(x)\right)(s)=\mathcal{M}\left((\log x)^{j} \varphi(x)\right)(s-\lambda)$, one may assume $\lambda=0$. As such, $v(x)=(\log x)^{j} \varphi(x)$.

\footnotetext{
I According to [MS3 Definition 6.4], a function in $\mathscr{S}_{\text {sis }}\left(\mathbb{R}_{+}\right)$is said to have a simple singularity at zero. Thus the subscript "sis" stands for "simple singularity".
} 
Let $A \in \mathbb{N}$. We have for $\mathfrak{R e} s>0$

$$
\begin{aligned}
\mathcal{M} v(s)= & \int_{0}^{1}(\log x)^{j}\left(\varphi(x)-\sum_{\kappa=0}^{A} a_{\kappa} x^{\kappa}\right) x^{s-1} d x+\sum_{\kappa=0}^{A} \frac{(-)^{j} j ! a_{\kappa}}{(s+\kappa)^{j+1}} \\
& +\int_{1}^{\infty}(\log x)^{j} \varphi(x) x^{s-1} d x .
\end{aligned}
$$

Here, we have used

$$
\int_{0}^{1}(\log x)^{j} x^{s-1} d x=\frac{(-)^{j} j !}{s^{j+1}}, \quad \text { Re } s>0 .
$$

In view of $\varphi(x)-\sum_{\kappa=0}^{A} a_{\kappa} x^{\kappa}=O_{A}\left(x^{A+1}\right)$, the first integral in 2.3 converges in the halfplane $\{s: \mathfrak{R e} s>-A-1\}$. The last integral converges for all $s$ on the whole complex plane due to the rapid decay of $\varphi$. Thus $H(s)=\mathcal{M} v(s)$ admits a meromorphic extension onto $\{s: \mathfrak{R e} s>-A-1\}$ and, since $A$ was arbitrary, onto the whole complex plane, with poles of pure order $j+1$ at the points in $-\mathbb{N}$.

For any given $\alpha \in \mathbb{N}$, repeating partial integration $\alpha$ times to the defining integral of $\mathcal{M} v(s)$ yields

$$
(-)^{\alpha}(s)_{\alpha} \mathcal{M} v(s)=\mathcal{M} v^{(\alpha)}(s+\alpha) .
$$

In view of this, we first expand $\mathcal{M} v^{(\alpha)}(s+\alpha)$ according to the expansion of $v^{(\alpha)}(x)=$ $(d / d x)^{\alpha}\left((\log x)^{j} \varphi(x)\right)$. We then write each term in the expansion of $\mathcal{M} v^{(\alpha)}(s+\alpha)$ in the same fashion as (2.3) and apply (1.26) and 1.28) to estimate the first and the last integral respectively. We conclude that

$$
\mathcal{M} v(s) \ll j, \alpha, A, a, b \frac{1}{\left|(s)_{\alpha}\right|}\left(1+\sum_{\kappa=0}^{\alpha+A}\left(\frac{1}{|s+\kappa|}+\ldots+\frac{1}{|s+\kappa|^{j+1}}\right)\right),
$$

for all $s \in \mathbb{S}[a, b]$, with $b \geqslant a>-\alpha-A-1$. In particular, 2.2 is proven.

Let $H \in \mathscr{M}_{\mathrm{sis}}^{\lambda, j}$. Suppose that the principal part of $H(s)$ at $s=\lambda-\kappa$ is equal to $(-)^{j} j ! a_{\kappa}(s+\lambda+\kappa)^{-j-1}$ and that $H(s)$ satisfies the condition 2.2 .

Claim 2. If we denote by $v(x)$ the following integral

$$
v(x)=\frac{1}{2 \pi i} \int_{(\sigma)} H(s) x^{-s} d s, \quad \sigma>\operatorname{Re} \lambda,
$$

then all the derivatives of $\varphi(x)=x^{\lambda}(\log x)^{-j} v(x)$ satisfy the asymptotics in 1.27) at zero and rapidly decay at infinity.

Proof of Claim 2. Again, let us assume $\lambda=0$.

Let $1>\rho>0$. We left shift the contour of integration from $(\sigma)$ to $(-\rho)$. When moving across $s=0$, we obtain $a_{0}(\log x)^{j}$ in view of Cauchy's differentiation formula It follows that

$$
v(x)=a_{0}(\log x)^{j}+\frac{1}{2 \pi i} \int_{(-\rho)} H(s) x^{-s} d s .
$$

${ }^{\mathrm{II}}$ Recall Cauchy's differentiation formula,

$$
f^{(j)}(\zeta)=\frac{j !}{2 \pi i} \oint_{\partial \mathbb{B}_{r}(\zeta)} \frac{f(s)}{(s-\zeta)^{j+1}} d s,
$$

where $f$ is a holomorphic function on a neighborhood of the closed disc $\overline{\mathbb{B}}_{r}(\zeta)$ centered at $\zeta$, and the integral is taken counter-clockwise on the circle $\partial \mathbb{B}_{r}(\zeta)$. In the present situation, this formula is applied for $f(s)=x^{-s}$. 
Using (2.2) with $r$ small, say $r<\rho$, to estimate the above integral, we arrive at

$$
v(x)=a_{0}(\log x)^{j}+O\left(x^{\rho}\right)=(\log x)^{j}\left(a_{0}+O\left(x^{\rho}\right)\right), \text { as } x \rightarrow 0 .
$$

Thus $\varphi(x)=(\log x)^{-j} v(x)$ satisfies the asymptotic (1.27) with $\alpha=0$. For the general case $\alpha \in \mathbb{N}$, we have

$$
v^{(\alpha)}(x)=(-)^{\alpha} \frac{1}{2 \pi i} \int_{(\sigma)}(s)_{\alpha} H(s) x^{-s-\alpha} d s .
$$

Shifting the contour from $(\sigma)$ to $(-\alpha-\rho)$ and following the same lines of arguments as above, combined with some straightforward algebraic manipulations, one may show (1.27) by an induction.

We are left to show the Schwartz condition for $\varphi(x)=(\log x)^{-j} v(x)$, or equivalently, that for $v(x)$. Indeed, the bound $(1.28)$ for $v^{(\alpha)}(x)$ follows from right shifting the contour of the integral in (2.4) to the vertical line $(\beta)$ and applying the estimates in (2.2). Q.E.D.

2.1.2. The Spaces $\mathscr{S}_{\text {sis }}\left(\mathbb{R}_{+}\right)$and $\mathscr{M}_{\text {sis. }}$ Let $\lambda, \lambda^{\prime} \in \mathbb{C}$. We write $\lambda \leqslant_{1} \lambda^{\prime}$ if $\lambda^{\prime}-\lambda \in \mathbb{N}$ and $\lambda \sim_{1} \lambda^{\prime}$ if $\lambda^{\prime}-\lambda \in \mathbb{Z}$. Observe that " $\leqslant 1$ " and " $\sim_{1}$ " define an order relation and an equivalence relation on $\mathbb{C}$ respectively.

Define

$$
\mathscr{S}_{\text {sis }}\left(\mathbb{R}_{+}\right)=\sum_{\lambda \in \mathbb{C}} \sum_{j \in \mathbb{N}} x^{-\lambda}(\log x)^{j} \mathscr{S}\left(\overline{\mathbb{R}}_{+}\right),
$$

where the sum $\sum_{\lambda \in \mathbb{C}} \sum_{j \in \mathbb{N}}$ is in the algebraic sense. It is clear that $\lambda \leqslant_{1} \lambda^{\prime}$ if and only if $x^{-\lambda}(\log x)^{j} \mathscr{S}\left(\overline{\mathbb{R}}_{+}\right) \subseteq x^{-\lambda^{\prime}}(\log x)^{j} \mathscr{S}\left(\overline{\mathbb{R}}_{+}\right)$. One also observes that $x^{-\lambda}(\log x)^{j} \mathscr{S}\left(\overline{\mathbb{R}}_{+}\right) \cap$ $x^{-\lambda^{\prime}}(\log x)^{j^{\prime}} \mathscr{S}\left(\overline{\mathbb{R}}_{+}\right)=\mathscr{S}\left(\mathbb{R}_{+}\right)$if either $j \neq j^{\prime}$ or $\lambda \not_{1} \lambda^{\prime}$. Therefore,

$$
\mathscr{S}_{\text {sis }}\left(\mathbb{R}_{+}\right) / \mathscr{S}\left(\mathbb{R}_{+}\right)=\bigoplus_{\omega \in \mathbb{C} / \sim_{1}} \bigoplus_{j \in \mathbb{N}} \underset{\lambda \in \omega}{\lim _{\lambda \in \omega}}\left(x^{-\lambda}(\log x)^{j} \mathscr{S}\left(\overline{\mathbb{R}}_{+}\right)\right) / \mathscr{S}\left(\mathbb{R}_{+}\right) .
$$

Here the direct limit $\lim _{\lambda \in \omega}$ is taken on the totally ordered set $\left(\omega, \preccurlyeq_{1}\right)$ and may be simply viewed as the union $\vec{\bigcup}_{\lambda \in \omega}$. More precisely, each function $v \in \mathscr{S}_{\text {sis }}\left(\mathbb{R}_{+}\right)$can be expressed as a sum

$$
v(x)=v^{0}(x)+\sum_{\lambda \in \Lambda} \sum_{j=0}^{N} x^{-\lambda}(\log x)^{j} v_{\lambda, j}(x),
$$

with $\Lambda \subset \mathbb{C}$ a finite set such that $\lambda \nsim_{1} \lambda^{\prime}$ for any two distinct points $\lambda, \lambda^{\prime} \in \Lambda, N \in \mathbb{N}$, $v^{0} \in \mathscr{S}\left(\mathbb{R}_{+}\right)$and $v_{\lambda, j} \in \mathscr{S}\left(\overline{\mathbb{R}}_{+}\right)$. This expression is unique up to addition of Schwartz functions in $\mathscr{S}\left(\mathbb{R}_{+}\right)$.

On the other hand, we define the space $\mathscr{M}_{\text {sis }}$ of all meromorphic functions $H$ satisfying the following conditions,

- the poles of $H$ lie in a finite number of sets $\lambda-\mathbb{N}$,

- the orders of the poles of $H$ are uniformly bounded, and

- $H$ decays rapidly along vertical lines, uniformly on vertical strips.

Appealing to certain Gamma identities for the Gamma function in [MS3 Lemma 6.24], one may show, in the same way as [MS3 , Lemma 6.35], that

$$
\mathscr{M}_{\mathrm{sis}}=\sum_{\lambda \in \mathbb{C}} \sum_{j \in \mathbb{N}} \mathscr{M}_{\mathrm{sis}}^{\lambda, j}
$$


We have $\mathscr{M}_{\text {sis }}^{\lambda, j} \subseteq \mathscr{M}_{\text {sis }}^{\lambda^{\prime}, j}$ if and only if $\lambda \leqslant_{1} \lambda^{\prime}$, and $\mathscr{M}_{\text {sis }}^{\lambda, j} \cap \mathscr{M}_{\text {sis }}^{\lambda^{\prime}, j^{\prime}}=\mathscr{H}_{\text {rd }}$ if either $j \neq j^{\prime}$ or $\lambda \nsim \lambda^{\prime}$. Therefore

$$
\mathscr{M}_{\mathrm{sis}} / \mathscr{H}_{\mathrm{rd}}=\bigoplus_{\omega \in \mathbb{C} / \sim_{1}} \bigoplus_{j \in \mathbb{N}} \lim _{\lambda \in \omega} \mathscr{M}_{\mathrm{sis}}^{\lambda, j} / \mathscr{H}_{\mathrm{rd}}
$$

The following lemma is a direct consequence of Lemma 2.1 .

Lemma 2.2. The Mellin transform $\mathcal{M}$ is an isomorphism between $\mathscr{S}_{\text {sis }}\left(\mathbb{R}_{+}\right)$and $\mathscr{M}_{\text {sis }}$ which respects their decompositions 2.5) and 2.6.

2.1.3. More Refined Decompositions of $\mathscr{S}_{\text {sis }}\left(\mathbb{R}_{+}\right)$and $\mathscr{M}_{\text {sis. }}$. Alternatively, we define an order relation on $\mathbb{C}, \lambda \leqslant_{2} \lambda^{\prime}$ if $\lambda^{\prime}-\lambda \in 2 \mathbb{N}$, as well as an equivalence relation, $\lambda \sim_{2} \lambda^{\prime}$ if $\lambda^{\prime}-\lambda \in 2 \mathbb{Z}$.

Define $\mathscr{N}_{\text {sis }}^{\lambda, j}$ in the same way as $\mathscr{M}_{\text {sis }}^{\lambda, j}$ with $\lambda-\mathbb{N}$ replaced by $\lambda-2 \mathbb{N}$. Under the isomorphism via $\mathcal{M}$ in Lemma 2.1. $\mathscr{N}_{\text {sis }}^{\lambda, j}$ is then isomorphic to $x^{-\lambda}(\log x)^{j} \mathscr{S}_{0}\left(\overline{\mathbb{R}}_{+}\right)$.

According to [MS3, Lemma 6.35], we have the following decomposition,

$$
\mathscr{M}_{\text {sis }}^{\lambda, j} / \mathscr{H}_{\mathrm{rd}}=\mathscr{N}_{\text {sis }}^{\lambda, j} / \mathscr{H}_{\text {rd }} \oplus \mathscr{N}_{\text {sis }}^{\lambda-1, j} / \mathscr{H}_{\text {rd }} \text {. }
$$

Inserting this into 2.6, we obtain the following refined decomposition of $\mathscr{M}_{\text {sis }} / \mathscr{H}_{\mathrm{rd}}$

$$
\bigoplus_{\omega \in \mathbb{C} / \sim_{1}} \bigoplus_{j \in \mathbb{N}} \underset{\lambda \in \omega}{\lim _{\lambda \in \omega}}\left(\mathscr{N}_{\text {sis }}^{\lambda, j} / \mathscr{H}_{\mathrm{rd}} \oplus \mathscr{N}_{\mathrm{sis}}^{\lambda-1, j} / \mathscr{H}_{\mathrm{rd}}\right)=\bigoplus_{\omega \in \mathbb{C} / \sim_{2}} \bigoplus_{j \in \mathbb{N}} \underset{\lambda \in \omega}{\lim } \mathscr{N}_{\mathrm{sis}}^{\lambda, j} / \mathscr{H}_{\mathrm{rd}}
$$

Under the isomorphism via $\mathcal{M}$ in Lemma 2.2, the reflection of this refinement on the decomposition of $\mathscr{S}_{\text {sis }}\left(\mathbb{R}_{+}\right) / \mathscr{S}\left(\mathbb{R}_{+}\right)$is

$$
\bigoplus_{\omega \in \mathbb{C} / \sim 2} \bigoplus_{j \in \mathbb{N}} \lim _{\lambda \in \omega}\left(x^{-\lambda}(\log x)^{j} \mathscr{S}_{0}\left(\overline{\mathbb{R}}_{+}\right)\right) / \mathscr{S}\left(\mathbb{R}_{+}\right) .
$$

Lemma 2.3. We have the following refinements of the decompositions 2.5, 2.6,

$$
\begin{gathered}
\mathscr{S}_{\text {sis }}\left(\mathbb{R}_{+}\right) / \mathscr{S}\left(\mathbb{R}_{+}\right)=\bigoplus_{\omega \in \mathbb{C} / \sim_{2}} \bigoplus_{j \in \mathbb{N}} \underset{\lambda \in \omega}{\lim }\left(x^{-\lambda}(\log x)^{j} \mathscr{S}_{0}\left(\overline{\mathbb{R}}_{+}\right)\right) / \mathscr{S}\left(\mathbb{R}_{+}\right) . \\
\mathscr{M}_{\text {sis }} / \mathscr{H}_{\mathrm{rd}}=\bigoplus_{\omega \in \mathbb{C} / \sim_{2}} \bigoplus_{j \in \mathbb{N}} \underset{\lambda \in \omega}{\lim } \mathscr{N}_{\text {sis }}^{\lambda, j} / \mathscr{H}_{\mathrm{rd}} .
\end{gathered}
$$

The Mellin transform $\mathcal{M}$ respects these two decompositions.

Corollary 2.4. Let $\delta \in \mathbb{Z} / 2 \mathbb{Z}$ and $m \in \mathbb{Z}$, and recall the definitions of $\mathscr{S}_{\delta}\left(\overline{\mathbb{R}}_{+}\right)$and $\mathscr{S}_{m}\left(\overline{\mathbb{R}}_{+}\right)$in $\$ 1.3 .1$

(1). The Mellin transform $\mathcal{M}$ respects the following decompositions,

$$
\begin{gathered}
\mathscr{S}_{\text {sis }}\left(\mathbb{R}_{+}\right) / \mathscr{S}\left(\mathbb{R}_{+}\right)=\bigoplus_{\omega \in \mathbb{C} / \sim_{2}} \bigoplus_{j \in \mathbb{N}} \underset{\lambda \in \omega}{\lim }\left(x^{-\lambda}(\log x)^{j} \mathscr{S}_{\delta}\left(\overline{\mathbb{R}}_{+}\right)\right) / \mathscr{S}\left(\mathbb{R}_{+}\right), \\
\mathscr{M}_{\text {sis }} / \mathscr{H}_{\mathrm{rd}}=\bigoplus_{\omega \in \mathbb{C} / \sim_{2}} \bigoplus_{j \in \mathbb{N}} \underset{\lambda \in \omega}{\lim } \mathscr{N}_{\text {sis }}^{\lambda-\delta, j} / \mathscr{H}_{\mathrm{rd}} .
\end{gathered}
$$

(2). The Mellin transform $\mathcal{M}$ respects the following decompositions,

$$
\begin{gathered}
\mathscr{S}_{\text {sis }}\left(\mathbb{R}_{+}\right) / \mathscr{S}\left(\mathbb{R}_{+}\right)=\bigoplus_{\omega \in \mathbb{C} / \sim_{2}} \bigoplus_{j \in \mathbb{N}} \underset{\lambda \in \omega}{\lim }\left(x^{-\lambda}(\log x)^{j} \mathscr{S}_{m}\left(\overline{\mathbb{R}}_{+}\right)\right) / \mathscr{S}\left(\mathbb{R}_{+}\right), \\
\mathscr{M}_{\text {sis }} / \mathscr{H}_{\text {rd }}=\bigoplus_{\omega \in \mathbb{C} / \sim_{2}} \bigoplus_{j \in \mathbb{N}} \underset{\lambda \in \omega}{\lim } \mathscr{N}_{\text {sis }}^{\lambda-|m|, j} / \mathscr{H}_{\text {rd }} .
\end{gathered}
$$


Proof. These follow from Lemma 2.3 in conjunction with $x^{\delta} \mathscr{S}_{0}\left(\overline{\mathbb{R}}_{+}\right)=\mathscr{S}_{\delta}\left(\overline{\mathbb{R}}_{+}\right)$and $x^{|m|} \mathscr{S}_{0}\left(\overline{\mathbb{R}}_{+}\right)=\mathscr{S}_{m}\left(\overline{\mathbb{R}}_{+}\right)$.

Q.E.D.

Remark 2.5. Set $\lambda=0$ and $j=0$ in 2.77. It follows from the isomorphism $\mathcal{M}$ the decomposition as below,

$$
\mathscr{S}\left(\overline{\mathbb{R}}_{+}\right) / \mathscr{S}\left(\mathbb{R}_{+}\right)=\mathscr{S}_{0}\left(\overline{\mathbb{R}}_{+}\right) / \mathscr{S}\left(\mathbb{R}_{+}\right) \oplus x \mathscr{S}_{0}\left(\overline{\mathbb{R}}_{+}\right) / \mathscr{S}\left(\mathbb{R}_{+}\right) .
$$

Since $x \mathscr{S}_{0}\left(\overline{\mathbb{R}}_{+}\right)=\mathscr{S}_{1}\left(\overline{\mathbb{R}}_{+}\right)$, one obtains $\mathscr{S}\left(\overline{\mathbb{R}}_{+}\right)=\mathscr{S}_{0}\left(\overline{\mathbb{R}}_{+}\right)+\mathscr{S}_{1}\left(\overline{\mathbb{R}}_{+}\right)$and therefore $C^{\infty}\left(\overline{\mathbb{R}}_{+}\right)=C_{0}^{\infty}\left(\overline{\mathbb{R}}_{+}\right)+C_{1}^{\infty}\left(\overline{\mathbb{R}}_{+}\right)$. See Remark 1.6

2.2. The Spaces $\mathscr{S}_{\text {sis }}\left(\mathbb{R}^{\times}\right)$and $\mathscr{M}_{\text {sis }}^{\mathbb{R}}$. Following [MS3, (6.10)], we write $(\lambda, \delta) \preccurlyeq$ $\left(\lambda^{\prime}, \delta^{\prime}\right)$ if $\lambda^{\prime}-\lambda \in \mathbb{N}$ and $\lambda^{\prime}-\lambda \equiv \delta^{\prime}+\delta(\bmod 2)$ and $(\lambda, \delta) \sim\left(\lambda^{\prime}, \delta^{\prime}\right)$ if $\lambda^{\prime}-\lambda-\left(\delta^{\prime}+\delta\right) \in 2 \mathbb{Z}$. Again, these define an order relation and an equivalence relation on $\mathbb{C} \times \mathbb{Z} / 2 \mathbb{Z}$.

2.2.1. The Space $\mathscr{S}_{\text {sis }}\left(\mathbb{R}^{\times}\right)$. According to [MS3, Definition 6.4] and [MS3, Lemma 6.35], define

$$
\mathscr{S}_{\text {sis }}\left(\mathbb{R}^{\times}\right)=\sum_{\delta \in \mathbb{Z} / 2 \mathbb{Z}} \sum_{\lambda \in \mathbb{C}} \sum_{j \in \mathbb{N}} \operatorname{sgn}(x)^{\delta}|x|^{-\lambda}(\log |x|)^{j} \mathscr{S}(\mathbb{R}) .
$$

We have the following decomposition,

$$
\mathscr{S}_{\text {sis }}\left(\mathbb{R}^{\times}\right) / \mathscr{S}\left(\mathbb{R}^{\times}\right)=\underset{\omega \in \mathbb{C} \times \mathbb{Z} / 2 \mathbb{Z} / \sim}{\bigoplus_{j \in \mathbb{N}}} \underset{(\lambda, \delta) \in \omega}{\lim _{\vec{\epsilon}}}\left(\operatorname{sgn}(x)^{\delta}|x|^{-\lambda}(\log |x|)^{j} \mathscr{S}(\mathbb{R})\right) / \mathscr{S}\left(\mathbb{R}^{\times}\right) .
$$

It follows from $\operatorname{sgn}(x)|x| \mathscr{S}(\mathbb{R})=x \mathscr{S}(\mathbb{R}) \subset \mathscr{S}(\mathbb{R})$ that

$$
\mathscr{S}_{\text {sis }}\left(\mathbb{R}^{\times}\right) / \mathscr{S}\left(\mathbb{R}^{\times}\right)=\bigoplus_{\omega \in \mathbb{C} / \sim 2} \bigoplus_{j \in \mathbb{N}} \underset{\lambda \in \omega}{\lim }\left(|x|^{-\lambda}(\log |x|)^{j} \mathscr{S}(\mathbb{R})\right) / \mathscr{S}\left(\mathbb{R}^{\times}\right) .
$$

We let $\mathscr{S}_{\text {sis }}^{\delta}\left(\mathbb{R}^{\times}\right)$denote the space of functions $v \in \mathscr{S}_{\text {sis }}\left(\mathbb{R}^{\times}\right)$satisfying the parity condition 1.17). Clearly, $\mathscr{S}_{\text {sis }}\left(\mathbb{R}^{\times}\right)=\mathscr{S}_{\text {sis }}^{0}\left(\mathbb{R}^{\times}\right) \oplus \mathscr{S}_{\text {sis }}^{1}\left(\mathbb{R}^{\times}\right)$. Then,

$$
\mathscr{S}_{\text {sis }}^{\delta}\left(\mathbb{R}^{\times}\right) / \mathscr{S}_{\delta}\left(\mathbb{R}^{\times}\right)=\bigoplus_{\omega \in \mathbb{C} / \sim_{2}} \bigoplus_{j \in \mathbb{N}} \underset{\lambda \in \omega}{\lim }\left(|x|^{-\lambda}(\log |x|)^{j} \mathscr{S}_{\delta}(\mathbb{R})\right) / \mathscr{S}_{\delta}\left(\mathbb{R}^{\times}\right),
$$

where $\mathscr{S}_{\delta}(\mathbb{R})$ and $\mathscr{S}_{\delta}\left(\mathbb{R}^{\times}\right)$are defined in $\$ 1.3 .1$ and $\$ 1.3 .2$ respectively. Since $\mathscr{S}_{\delta}(\mathbb{R})=$ $\operatorname{sgn}(x)^{\delta} \mathscr{S}_{\delta}\left(\overline{\mathbb{R}}_{+}\right)$,

$$
\mathscr{S}_{\text {sis }}^{\delta}\left(\mathbb{R}^{\times}\right) / \mathscr{S}_{\delta}\left(\mathbb{R}^{\times}\right)=\bigoplus_{\omega \in \mathbb{C} / \sim 2} \bigoplus_{j \in \mathbb{N}} \underset{\lambda \in \omega}{\lim }\left(\operatorname{sgn}(x)^{\delta}|x|^{-\lambda}(\log |x|)^{j} \mathscr{S}_{\delta}\left(\overline{\mathbb{R}}_{+}\right)\right) / \mathscr{S}_{\delta}\left(\mathbb{R}^{\times}\right) .
$$

Then, $|x|^{-\delta} \mathscr{S}_{\delta}\left(\overline{\mathbb{R}}_{+}\right)=\mathscr{S}_{0}\left(\overline{\mathbb{R}}_{+}\right)$together with $\mathscr{S}_{0}\left(\overline{\mathbb{R}}_{+}\right) / \mathscr{S}\left(\mathbb{R}_{+}\right) \oplus|x| \mathscr{S}_{0}\left(\overline{\mathbb{R}}_{+}\right) / \mathscr{S}\left(\mathbb{R}_{+}\right)=$ $\mathscr{S}\left(\overline{\mathbb{R}}_{+}\right) / \mathscr{S}\left(\mathbb{R}_{+}\right)$(see Remark 2.5 yields

$$
\mathscr{S}_{\text {sis }}^{\delta}\left(\mathbb{R}^{\times}\right) / \mathscr{S}_{\delta}\left(\mathbb{R}^{\times}\right)=\bigoplus_{\omega \in \mathbb{C} / \sim_{1}} \underset{j \in \mathbb{N}}{\bigoplus} \underset{\lambda \in \omega}{\lim }\left(\operatorname{sgn}(x)^{\delta}|x|^{-\lambda}(\log |x|)^{j} \mathscr{S}\left(\overline{\mathbb{R}}_{+}\right)\right) / \mathscr{S}_{\delta}\left(\mathbb{R}^{\times}\right) .
$$

In particular,

$$
\mathscr{S}_{\text {sis }}^{\delta}\left(\mathbb{R}^{\times}\right)=\operatorname{sgn}(x)^{\delta} \mathscr{S}_{\text {sis }}\left(\mathbb{R}_{+}\right)=\left\{\operatorname{sgn}(x)^{\delta} v(|x|): v \in \mathscr{S}_{\text {sis }}\left(\mathbb{R}_{+}\right)\right\} .
$$

2.2.2. The Space $\mathscr{M}_{\text {sis }}^{\mathbb{R}}$. We simply define $\mathscr{M}_{\text {sis }}^{\mathbb{R}}=\mathscr{M}_{\text {sis }} \times \mathscr{M}_{\text {sis }}$. 
2.2.3. Isomorphism between $\mathscr{S}_{\text {sis }}\left(\mathbb{R}^{\times}\right)$and $\mathscr{M}_{\text {sis }}^{\mathbb{R}}$ via the Mellin Transform $\mathcal{M}_{\mathbb{R}}$. Let $v \in \mathscr{S}_{\text {sis }}\left(\mathbb{R}^{\times}\right)$. Since $v_{\delta} \in \mathscr{S}_{\text {sis }}\left(\overline{\mathbb{R}}_{+}\right)$, the identity $\mathcal{M}_{\delta} v(s)=2 \mathcal{M} v_{\delta}(s)$ in (1.42) extends the definition of the Mellin transform $\mathcal{M}_{\delta}$ onto the space $\mathscr{S}_{\text {sis }}\left(\mathbb{R}^{\times}\right)$. Therefore, as a consequence of Lemma 2.1,2.2 and Corollary 2.4(1), the following lemma is readily established.

Lemma 2.6. For $\delta \in \mathbb{Z} / 2 \mathbb{Z}$, the Mellin transform $\mathcal{M}_{\delta}$ establishes an isomorphism between the spaces $\mathscr{S}_{\text {sis }}^{\delta}\left(\mathbb{R}^{\times}\right)$and $\mathscr{M}_{\text {sis }}$ which respects their decompositions (2.15) and 2.6) as well as 2.14) and 2.11. Therefore, $\mathcal{M}^{\mathbb{R}}=\left(\mathcal{M}_{0}, \mathcal{M}_{1}\right)$ establishes an isomorphism between $\mathscr{S}_{\text {sis }}\left(\mathbb{R}^{\times}\right)=\mathscr{S}_{\text {sis }}^{0}\left(\mathbb{R}^{\times}\right) \oplus \mathscr{S}_{\text {sis }}^{1}\left(\mathbb{R}^{\times}\right)$and $\mathscr{M}_{\text {sis }}^{\mathbb{R}}=\mathscr{M}_{\text {sis }} \times \mathscr{M}_{\text {sis }}$.

2.2.4. An Alternative Decomposition of $\mathscr{S}_{\text {sis }}^{\delta}\left(\mathbb{R}^{\times}\right)$. The following lemma follows from Corollary 2.4 (1) (compare [MS3, Corollary 6.17]).

Lemma 2.7. Let $\delta \in \mathbb{Z} / 2 \mathbb{Z}$. The Mellin transform $\mathcal{M}_{\delta}$ respects the following decompositions,

$$
\begin{aligned}
& \mathscr{S}_{\text {sis }}^{\delta}\left(\mathbb{R}^{\times}\right) / \mathscr{S}_{\delta}\left(\mathbb{R}^{\times}\right) \\
& =\underset{\omega \in \mathbb{C} \times \mathbb{Z} / 2 \mathbb{Z} / \sim}{\bigoplus_{j \in \mathbb{N}}}{\underset{(i m}{(\lambda, \epsilon) \epsilon \omega}}_{\lim }\left(\operatorname{sgn}(x)^{\epsilon}|x|^{-\lambda}(\log |x|)^{j} \mathscr{S}_{\epsilon+\delta}(\mathbb{R})\right) / \mathscr{S}_{\delta}\left(\mathbb{R}^{\times}\right), \\
& \mathscr{M}_{\text {sis }} / \mathscr{H}_{\mathrm{rd}}=\bigoplus_{\omega \in \mathbb{C} \times \mathbb{Z} / 2 \mathbb{Z} / \sim} \bigoplus_{j \in \mathbb{N}} \underset{(\lambda, \epsilon) \in \omega}{\lim _{\text {sis }}} \mathscr{N}^{\lambda-(\epsilon+\delta), j} / \mathscr{H}_{\mathrm{rd}} .
\end{aligned}
$$

2.3. The Spaces $\mathscr{S}_{\text {sis }}\left(\mathbb{C}^{\times}\right)$and $\mathscr{M}_{\text {sis }}^{\mathbb{C}}$. We write $(\lambda, m) \leqslant\left(\lambda^{\prime}, m^{\prime}\right)$ if $\lambda^{\prime}-\lambda \in \mid m^{\prime}-$ $m \mid+2 \mathbb{N}$ and $(\lambda, m) \sim\left(\lambda^{\prime}, m^{\prime}\right)$ if $\lambda^{\prime}-\lambda-\left|m^{\prime}-m\right| \in 2 \mathbb{Z}$. These define an order relation and an equivalence relation on $\mathbb{C} \times \mathbb{Z}$.

2.3.1. The Space $\mathscr{S}_{\text {sis }}\left(\mathbb{C}^{\times}\right)$. In parallel to $\$ 2.2 .1$, we first define

$$
\mathscr{S}_{\text {sis }}\left(\mathbb{C}^{\times}\right)=\sum_{m \in \mathbb{Z}} \sum_{\lambda \in \mathbb{C}} \sum_{j \in \mathbb{N}}[z]^{-m}|z|^{-\lambda}(\log |z|)^{j} \mathscr{S}(\mathbb{C}) .
$$

We have the following decomposition,

$$
\mathscr{S}_{\text {sis }}\left(\mathbb{C}^{\times}\right) / \mathscr{S}\left(\mathbb{C}^{\times}\right)=\bigoplus_{\omega \in \mathbb{C} \times \mathbb{Z} / \sim} \bigoplus_{j \in \mathbb{N}} \underset{(\lambda, m) \in \omega}{\lim _{\vec{m}}}\left([z]^{-m}|z|^{-\lambda}(\log |z|)^{j} \mathscr{S}(\mathbb{C})\right) / \mathscr{S}\left(\mathbb{C}^{\times}\right) .
$$

It follows from $[z]|z| \mathscr{S}(\mathbb{C})=z \mathscr{S}(\mathbb{C}) \subset \mathscr{S}(\mathbb{C})$ that

$$
\mathscr{S}_{\text {sis }}\left(\mathbb{C}^{\times}\right) / \mathscr{S}\left(\mathbb{C}^{\times}\right)=\bigoplus_{\omega \in \mathbb{C} / \sim_{2}} \bigoplus_{j \in \mathbb{N}} \underset{\lambda \in \omega}{\lim }\left(|z|^{-\lambda}(\log |z|)^{j} \mathscr{S}(\mathbb{C})\right) / \mathscr{S}\left(\mathbb{C}^{\times}\right) .
$$

We let $\mathscr{S}_{\text {sis }}^{m}\left(\mathbb{C}^{\times}\right)$denote the space of functions $v \in \mathscr{S}_{\text {sis }}\left(\mathbb{C}^{\times}\right)$satisfying 1.21]. Then,

$$
\mathscr{S}_{\text {sis }}^{m}\left(\mathbb{C}^{\times}\right) / \mathscr{S}_{m}\left(\mathbb{C}^{\times}\right)=\bigoplus_{\omega \in \mathbb{C} / \sim_{2}} \bigoplus_{j \in \mathbb{N}} \underset{\lambda \in \omega}{\lim }\left(|z|^{-\lambda}(\log |z|)^{j} \mathscr{S}_{m}(\mathbb{C})\right) / \mathscr{S}_{m}\left(\mathbb{C}^{\times}\right),
$$

where $\mathscr{S}_{m}(\mathbb{C})$ and $\mathscr{S}_{m}\left(\mathbb{C}^{\times}\right)$are defined in $\$ 1.3 .1$ and $\$ 1.3 .2$ respectively. Since $\mathscr{S}_{m}(\mathbb{C})=$ $[z]^{m} \mathscr{S}_{m}\left(\overline{\mathbb{R}}_{+}\right)$,

$$
\mathscr{S}_{\text {sis }}^{m}\left(\mathbb{C}^{\times}\right) / \mathscr{S}_{m}\left(\mathbb{C}^{\times}\right)=\bigoplus_{\omega \in \mathbb{C} / \sim_{2}} \bigoplus_{j \in \mathbb{N}} \underset{\lambda \in \omega}{\lim _{\lambda \in \omega}}\left([z]^{m}|z|^{-\lambda}(\log |z|)^{j} \mathscr{S}_{m}\left(\overline{\mathbb{R}}_{+}\right)\right) / \mathscr{S}_{m}\left(\mathbb{C}^{\times}\right) .
$$

Then, $|z|^{-|m|} \mathscr{S}_{m}\left(\overline{\mathbb{R}}_{+}\right)=\mathscr{S}_{0}\left(\overline{\mathbb{R}}_{+}\right)$together with $\mathscr{S}_{0}\left(\overline{\mathbb{R}}_{+}\right) / \mathscr{S}\left(\mathbb{R}_{+}\right) \oplus|z| \mathscr{S}_{0}\left(\overline{\mathbb{R}}_{+}\right) / \mathscr{S}\left(\mathbb{R}_{+}\right)=$ $\mathscr{S}\left(\overline{\mathbb{R}}_{+}\right) / \mathscr{S}\left(\mathbb{R}_{+}\right)$yields

$$
\mathscr{S}_{\text {sis }}^{m}\left(\mathbb{C}^{\times}\right) / \mathscr{S}_{m}\left(\mathbb{C}^{\times}\right)=\bigoplus_{\omega \in \mathbb{C} / \sim 1} \bigoplus_{j \in \mathbb{N}} \underset{\lambda \in \omega}{\lim _{\lambda}}\left([z]^{m}|z|^{-\lambda}(\log |z|)^{j} \mathscr{S}\left(\overline{\mathbb{R}}_{+}\right)\right) / \mathscr{S}_{m}\left(\mathbb{C}^{\times}\right) .
$$


In particular,

$$
\mathscr{S}_{\text {sis }}^{m}\left(\mathbb{C}^{\times}\right)=[z]^{m} \mathscr{S}_{\text {sis }}\left(\mathbb{R}_{+}\right)=\left\{[z]^{m} v(|z|): v \in \mathscr{S}_{\text {sis }}\left(\mathbb{R}_{+}\right)\right\} .
$$

2.3.2. The Space $\mathscr{M}_{\text {sis }}^{\mathbb{C}}$. For $\lambda \in \mathbb{C}$ and $j \in \mathbb{N}$, we define the space $\mathscr{N}_{\text {sis }}^{\mathbb{C}, \lambda, j}$ of all sequences $\left\{H_{m}(s)\right\}$ of meromorphic functions such that

- the only singularities of $H_{m}(s)$ are poles of pure order $j+1$ at the points in $\lambda-|m|-2 \mathbb{N}$,

- Each $H_{m}(s)$ decays rapidly along vertical lines, uniformly on vertical strips (see (2.1), and

- $H_{m}(s)$ also decays rapidly with respect to $m$, uniformly on vertical strips, in the sense that for any given $\alpha, A \in \mathbb{N}$ and vertical strip $\mathbb{S}[a, b]$,

$H_{m}(s) \ll_{\lambda, j, \alpha, A, a, b}(|m|+1)^{-A}(|\mathfrak{I m} s|+1)^{-\alpha}$ for all $s \in \mathbb{S}[a, b]$, if $|m|>\Re e \lambda-a$.

Observe that the first two conditions amount to $H_{m} \in \mathscr{N}_{\text {sis }}^{\lambda-|m|, j}$. Therefore, $\mathscr{N}_{\text {sis }}^{\mathbb{C}, \lambda, j} \subset$ $\prod_{m \in \mathbb{Z}} \mathscr{N}_{\text {sis }}^{\lambda-|m|, j}$.

Define the space $\mathscr{M}_{\text {sis }}^{\mathbb{C}}$ of all sequences $\left\{H_{m}\right\}$ of meromorphic functions such that

- the poles of each $H_{m}$ lie in $\lambda-|m|-2 \mathbb{N}$, for a finite number of $\lambda$,

- the orders of the poles of $H_{m}$ are uniformly bounded,

- Each $H_{m}$ decays rapidly along vertical lines, uniformly on vertical strips, and

- $H_{m}$ decays rapidly with respect to $m$, uniformly on vertical strips.

Using the refined Stirling's asymptotic formula (1.13) in place of [MS3, (6.22)] and the following bound in place of [MS3 (6.23)]

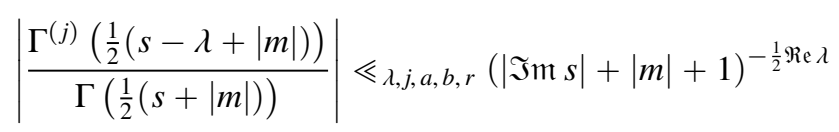

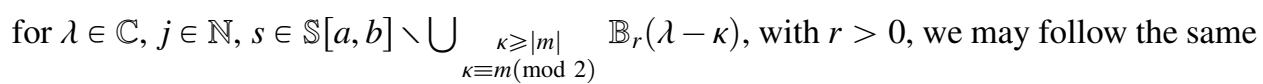
lines of the proofs of [MS3 Lemma 6.24] and [MS3, Lemma 6.35] to show that

$$
\mathscr{M}_{\mathrm{sis}}^{\mathbb{C}}=\sum_{\lambda \in \mathbb{C}} \sum_{j \in \mathbb{N}} \mathscr{N}_{\mathrm{sis}}^{\mathbb{C}, \lambda, j}
$$

and consequently

$$
\mathscr{M}_{\mathrm{sis}}^{\mathbb{C}} / \mathscr{H}_{\mathrm{rd}}^{\mathbb{C}}=\bigoplus_{\omega \in \mathbb{C} / \sim 2} \bigoplus_{j \in \mathbb{N}} \underset{\lambda \in \omega}{\lim _{\lambda \in \omega}} \mathscr{N}_{\mathrm{sis}}^{\mathbb{C}, \lambda, j} / \mathscr{H}_{\mathrm{rd}}^{\mathbb{C}}
$$

2.3.3. Isomorphism between $\mathscr{S}_{\text {sis }}\left(\mathbb{C}^{\times}\right)$and $\mathscr{M}_{\text {sis }}^{\mathbb{C}}$ via the Mellin Transform $\mathcal{M}_{\mathbb{C}}$. For $v \in \mathscr{S}_{\text {sis }}\left(\mathbb{C}^{\times}\right)$, its $m$-th Fourier coefficient $v_{m}$ is a function in $\mathscr{S}_{\text {sis }}\left(\overline{\mathbb{R}}_{+}\right)$. Hence the identity $\mathcal{M}_{-m} v(s)=4 \pi \mathcal{N} v_{m}(s)$ in 1.43 extends the definition of the Mellin transform $\mathcal{M}_{-m}$ onto the space $\mathscr{S}_{\text {sis }}\left(\mathbb{C}^{\times}\right)$.

Lemma 2.8. For $m \in \mathbb{Z}$, the Mellin transform $\mathcal{M}_{-m}$ establishes an isomorphism between the spaces $\mathscr{S}_{\mathrm{sis}}^{m}\left(\mathbb{C}^{\times}\right)$and $\mathscr{M}_{\mathrm{sis}}$ which respects their decompositions (2.20) and 2.6) as well as 2.19] and 2.13). Furthermore, $\mathcal{M}_{\mathbb{C}}=\prod_{m \in \mathbb{Z}} \mathcal{M}_{-m}$ establishes an isomorphism between $|z|^{-\lambda}(\log |z|)^{j} \mathscr{S}(\mathbb{C})$ and $\mathscr{N}_{\text {sis }}^{\mathbb{C}, \lambda, j}$ for any $\lambda \in \mathbb{C}$ and $j \in \mathbb{N}$, and hence an isomorphism between $\mathscr{S}_{\text {sis }}\left(\mathbb{C}^{\times}\right)$and $\mathscr{M}_{\text {sis }}^{\mathbb{C}}$ which respects their decompositions 2.18) and (2.22). 
Proof. For $v \in \mathscr{S}_{\text {sis }}^{m}\left(\mathbb{C}^{\times}\right)$, one has $v\left(x e^{i \phi}\right)=e^{i m \phi} v_{m}(x)$ and $v_{m} \in \mathscr{S}_{\text {sis }}\left(\overline{\mathbb{R}}_{+}\right)$. Thus the first assertion follows immediately from Lemma 2.2 and Corollary 2.4 (2).

Now let $\varphi \in \mathscr{S}(\mathbb{C})$ and $v(z)=|z|^{-\lambda}(\log |z|)^{j} \varphi(z)$. Clearly, their $m$-th Fourier coefficients are related by $v_{m}(x)=x^{-\lambda}(\log x)^{j} \varphi_{m}(x)$. Since $\varphi_{m} \in \mathscr{S}_{m}\left(\overline{\mathbb{R}}_{+}\right)$, it follows from Corollary 2.4 (2) that $H_{m}=\mathcal{M}_{-m} v=4 \pi \mathcal{M} v_{m}$ lies in $\mathscr{N}_{\text {sis }}^{\lambda-|m|, j}$, and therefore we are left to show 2.21). Recall that in the proof of Lemma 2.1 we turned to verify $(2.2)$ instead of (2.1). Likewise, it is more convenient to verify the following equivalent statement of (2.21),

$$
\begin{aligned}
& \text { for any given } \alpha, A \in \mathbb{N}, b \geqslant a>\mathfrak{R e} \lambda-\alpha-A-1, \\
& H_{m}(s) \ll_{\lambda, j, \alpha, A, a, b}(|m|+1)^{-A}(|\mathfrak{I m} s|+1)^{-\alpha} \text { for all } s \in \mathbb{S}[a, b], \text { if }|m|>\alpha+A .
\end{aligned}
$$

According to Lemma $1.5(3.1), \varphi_{m}$ satisfies the conditions 1.32 1.35. Suppose $|m|>$ $\alpha+A$. One directly applies (1.32) and (1.35) to bound the following integral by a constant multiple of $(|m|+1)^{-A}$,

$$
(-)^{\alpha}(s-\lambda)_{\alpha} \mathcal{M} v_{m}(s)=\int_{0}^{\infty} \frac{d^{\alpha}}{d x^{\alpha}}\left((\log x)^{j} \varphi_{m}(x)\right) x^{s-\lambda+\alpha-1} d x .
$$

This proves 2.23 for $H_{m}=4 \pi \mathcal{M} v_{m}$. Therefore, the sequence $\left\{\mathcal{M}_{-m} v\right\}$ belongs to $\mathscr{N}_{\text {sis }}^{\mathbb{C}, \lambda, j}$.

Conversely, let $\left\{H_{m}\right\} \in \mathscr{N}_{\text {sis }}^{\mathbb{C}, \lambda, j}$, and let $4 \pi v_{m}$ be the Mellin inversion of $H_{m}$,

$$
v_{m}(x)=\frac{1}{8 \pi^{2} i} \int_{(\sigma)} H_{m}(s) x^{-s} d s, \quad \sigma>\Re \mathrm{e} \lambda-|m| .
$$

Since $H_{m} \in \mathscr{N}_{\text {sis }}^{\lambda-|m|, j}$, Corollary 2.4 (2) implies that $v_{m}(x) \in x^{-\lambda}(\log x)^{j} \mathscr{S}_{m}\left(\overline{\mathbb{R}}_{+}\right)$and hence $\varphi_{m}(x)=x^{\lambda}(\log x)^{-j} v_{m}(x)$ lies in $\mathscr{S}_{m}\left(\overline{\mathbb{R}}_{+}\right)$. This proves 1.34). Similar to the proof of Lemma 2.1. right shifting of the contour of integration combined with 2.23 yields (1.32), whereas left shifting combined with (2.23) yields 1.35$]^{\text {III] }}$

The proof of the second assertion is completed.

Q.E.D.

2.3.4. An Alternative Decomposition of $\mathscr{S}_{\mathrm{sis}}^{m}\left(\mathbb{C}^{\times}\right)$. The following lemma follows from Corollary 2.4 (2).

Lemma 2.9. Let $m \in \mathbb{Z}$. The Mellin transform $\mathcal{M}_{-m}$ respects the following decompositions,

$$
\begin{gathered}
\mathscr{S}_{\text {sis }}^{m}\left(\mathbb{C}^{\times}\right) / \mathscr{S}_{m}\left(\mathbb{C}^{\times}\right) \\
=\bigoplus_{\omega \in \mathbb{C} \times \mathbb{Z} / \sim} \bigoplus_{j \in \mathbb{N}} \underset{(\lambda, k) \in \omega}{\lim _{\overrightarrow{1}}}\left([z]^{-k}|z|^{-\lambda}(\log |z|)^{j} \mathscr{S}_{m+k}(\mathbb{C})\right) / \mathscr{S}_{m}\left(\mathbb{C}^{\times}\right), \\
\mathscr{M}_{\mathrm{sis}} / \mathscr{H}_{\mathrm{rd}}=\bigoplus_{\omega \in \mathbb{C} \times \mathbb{Z} / \sim} \underset{j \in \mathbb{N}}{\bigoplus_{(\lambda, k) \in \omega}} \underset{\lim _{\text {sis }}}{\mathscr{N}_{\mathrm{sid}}^{\lambda-|m+k|, j} / \mathscr{H}_{\mathrm{rd}} .}
\end{gathered}
$$

\section{Hankel Transforms and Bessel Kernels}

This section is arranged as follows. We start with the type of Hankel transforms over $\mathbb{R}_{+}$whose kernels are the Bessel functions that will be extensively studied in Chapter 2 . After this, we introduce two auxiliary Hankel transforms and Bessel kernels over $\mathbb{R}_{+}$. Finally, we proceed to construct and study Hankel transforms and their Bessel kernels over $\mathbb{F}^{\times}$, with $\mathbb{F}=\mathbb{R}, \mathbb{C}$.

\footnotetext{
III Actually, $O_{\alpha, A}\left((|m|+1)^{-A} x^{A+1}\right)$ in 1.35 should be replaced by $O_{\alpha, A, \rho}\left((|m|+1)^{-A} x^{A+\rho}\right), 1>\rho>$ 0 . Moreover, one observes that the left contour shift here does not cross any pole.
} 

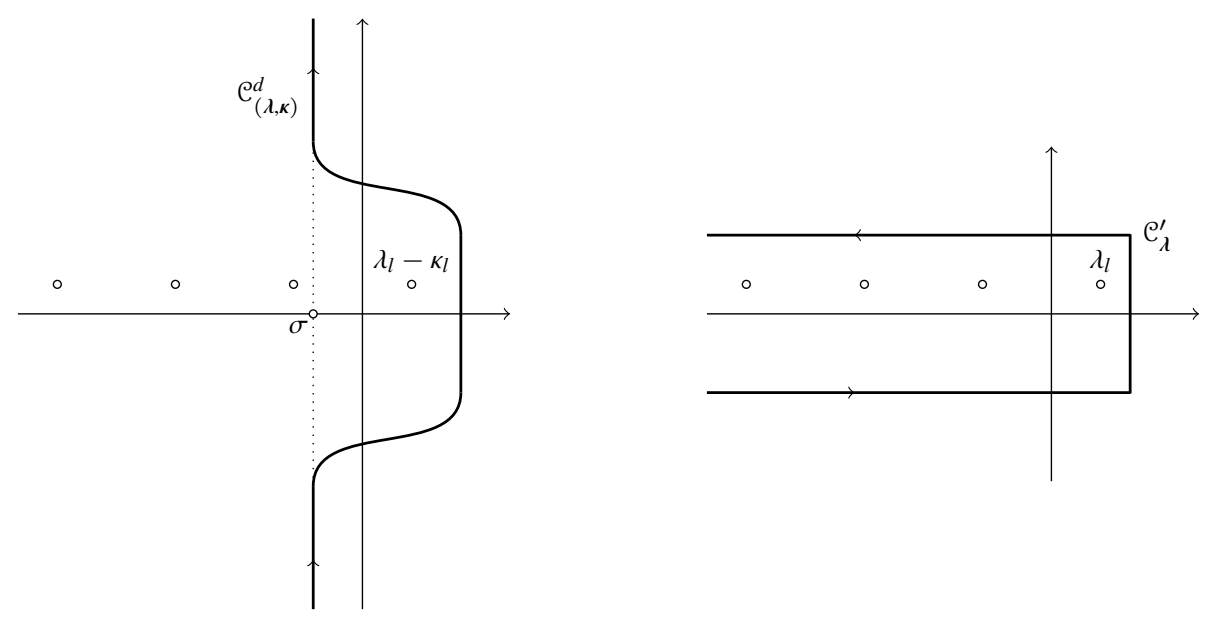

FIGURE 1. $\mathcal{C}_{(\lambda, \kappa)}^{d}$ and $\mathcal{C}_{\lambda}^{\prime}$

DefinItion 3.1. Let $(\mathbb{X}, \preccurlyeq)$ be an ordered set satisfying the condition that

$$
\text { “ } \lambda \leqslant \lambda^{\prime} \text { or } \lambda^{\prime} \leqslant \lambda \text { " is an equivalence relation. }
$$

We denote the above equivalence relation by $\lambda \sim \lambda^{\prime}$. Given $\lambda=\left(\lambda_{1}, \ldots, \lambda_{n}\right) \in \mathbb{X}^{n}$, the set $\{1, \ldots n\}$ is partitioned into several pair-wise disjoint subsets $L_{\alpha}, \alpha=1, \ldots, A$, such that

$$
\lambda_{l} \sim \lambda_{l^{\prime}} \text { if and only if } l, l^{\prime} \text { are in the same } L_{\alpha} .
$$

Each $\Lambda^{\alpha}=\left\{\lambda_{l}\right\}_{l \in L_{0}}{ }^{\mathbb{I}}$ is a totally ordered set. Let $B_{\alpha}=\left|\Lambda^{\alpha}\right|$ and label the elements of $\Lambda^{\alpha}$ in the descending order, $\lambda_{\alpha, 1}>\ldots>\lambda_{\alpha, B_{\alpha}}$. For $\lambda_{\alpha, \beta} \in \Lambda^{\alpha}$, let $M_{\alpha, \beta}$ denote the multiplicity of $\lambda_{\alpha, \beta}$ in $\lambda$, that is, $M_{\alpha, \beta}=\left|\left\{l: \lambda_{l}=\lambda_{\alpha, \beta}\right\}\right|$, and define $N_{\alpha, \beta}=\sum_{\gamma=1}^{\beta} M_{\alpha, \gamma}=\left|\left\{l: \lambda_{\alpha, \beta} \leqslant \lambda_{l}\right\}\right|$.

$\lambda$ is called generic if $\lambda_{l} \nsim \lambda_{l^{\prime}}$ for any $l \neq l^{\prime}$.

We recall that the ordered sets $\left(\mathbb{C}, \leqslant_{1}\right),\left(\mathbb{C}, \leqslant_{2}\right),(\mathbb{C} \times \mathbb{Z} / 2 \mathbb{Z}, \preccurlyeq)$ and $(\mathbb{C} \times \mathbb{Z}, \preccurlyeq)$ defined in $\$ 2$ all satisfy 3.1 .

Definition 3.2. Let $d=1$ or $2, \lambda \in \mathbb{C}^{n}$ and $\boldsymbol{\kappa} \in \mathbb{N}^{n}$. Put $\sigma<\frac{d}{2}+\frac{1}{n}(\mathfrak{R e}|\lambda|-1)$ and choose a contour $\mathrm{C}_{(\lambda, \kappa)}^{d}$ (see Figure 1p such that

- $\mathcal{C}_{(\lambda, \kappa)}^{d}$ is upward directed from $\sigma-i \infty$ to $\sigma+i \infty$,

- all the sets $\lambda_{l}-\kappa_{l}-\mathbb{N}$ lie on the left side of $\mathcal{C}_{(\lambda, \kappa)}$, and

- if $s \in \mathcal{C}_{(\lambda, \kappa)}^{d}$ and $|\mathfrak{I m} s|$ is sufficiently large, say $|\mathfrak{I m} s|-\max \left\{\left|\mathfrak{I m} \lambda_{l}\right|\right\} \gg 1$, then Re $s=\sigma$.

For $\lambda \in \mathbb{C}$, we denote $\mathcal{C}_{\lambda}=\mathcal{C}_{(\lambda, \boldsymbol{0})}^{1}$. For $(\boldsymbol{\mu}, \boldsymbol{\delta}) \in \mathbb{C}^{n} \times(\mathbb{Z} / 2 \mathbb{Z})^{n}$, we denote $\mathcal{C}_{(\boldsymbol{\mu}, \boldsymbol{\delta})}=\mathcal{C}_{(\boldsymbol{\mu}, \boldsymbol{\delta})}^{1}$. For $(\boldsymbol{\mu}, \boldsymbol{m}) \in \mathbb{C}^{n} \times \mathbb{Z}^{n}$, we denote $\mathcal{C}_{(\boldsymbol{\mu}, \boldsymbol{m})}=\frac{1}{2} \cdot \mathcal{C}_{(2 \boldsymbol{\mu},\|\boldsymbol{m}\|)}^{2}$.

Definition 3.3. For $\lambda \in \mathbb{C}^{n}$, choose a contour $\mathcal{C}_{\lambda}^{\prime}$ illustrated in Figure 1 such that

- $\mathcal{C}_{\lambda}^{\prime}$ starts from and returns to $-\infty$ counter-clockwise,

- $\mathcal{C}_{\lambda}^{\prime}$ consists two horizontal infinite half lines,

- $\mathcal{C}_{\lambda}^{\prime}$ encircles all the sets $\lambda_{l}-\mathbb{N}$, and

- $\mathfrak{I m} s \ll \max \left\{\left|\mathfrak{I m} \lambda_{l}\right|\right\}+1$ for all $s \in \mathcal{C}_{\lambda}^{\prime}$.

\footnotetext{
$\mathrm{IV}_{\text {Here, }}\left\{\lambda_{l}\right\}_{l \in L_{\alpha}}$ is considered as a set, namely, $\lambda_{l}$ are counted without multiplicity.
} 
3.1. The Hankel Transform $\mathcal{H}_{(\varsigma, \lambda)}$ and the Bessel Function $J(x ; \boldsymbol{\varsigma}, \lambda)$.

3.1.1. The Definition of $\mathcal{H}_{(\boldsymbol{s}, \lambda)}$. Consider the ordered set $\left(\mathbb{C}, \leqslant_{1}\right)$. For $\lambda \in \mathbb{C}^{n}$, let notations $\lambda_{\alpha, \beta}, B_{\alpha}, M_{\alpha, \beta}$ and $N_{\alpha, \beta}$ be as in Definition 3.1. We define the following subspace of $\mathscr{S}_{\text {sis }}\left(\mathbb{R}_{+}\right)$,

$$
\mathscr{S}_{\text {sis }}^{\lambda}\left(\mathbb{R}_{+}\right)=\sum_{\alpha=1}^{A} \sum_{\beta=1}^{B_{\alpha}} \sum_{j=0}^{N_{\alpha \beta \beta}-1} x^{-\lambda_{\alpha \beta \beta}}(\log x)^{j} \mathscr{S}\left(\overline{\mathbb{R}}_{+}\right) .
$$

Proposition 3.4. Let $(\boldsymbol{s}, \lambda) \in\{+,-\}^{n} \times \mathbb{C}^{n}$. Suppose $v \in \mathscr{S}\left(\mathbb{R}_{+}\right)$. Then there exists a unique function $\Upsilon \in \mathscr{S}_{\text {sis }}^{\lambda}\left(\mathbb{R}_{+}\right)$satisfying the following identity,

$$
\mathcal{M} \Upsilon(s)=G(s ; \boldsymbol{s}, \lambda) \mathcal{M} v(1-s) .
$$

We call $\Upsilon$ the Hankel transform of $v$ over $\mathbb{R}_{+}$of index $(\boldsymbol{s}, \lambda)$ and write $\mathcal{H}_{(\boldsymbol{s}, \lambda)} v=\Upsilon$.

Proof. Recall the definition of $G(s ; \boldsymbol{\varsigma}, \lambda)$ given by $1.1,1.2$,

$$
G(s ; \boldsymbol{\varsigma}, \lambda)=e\left(\frac{\sum_{l=1}^{n} \varsigma_{l}\left(s-\lambda_{l}\right)}{4}\right) \prod_{l=1}^{n} \Gamma\left(s-\lambda_{l}\right) .
$$

The product in the above expression may be rewritten as below

$$
\prod_{\alpha=1}^{A} \prod_{\beta=1}^{B_{\alpha}} \Gamma\left(s-\lambda_{\alpha, \beta}\right)^{M_{\alpha, \beta}} .
$$

Thus the singularities of $G(s ; \boldsymbol{\varsigma}, \lambda)$ are poles at the points in $\lambda_{\alpha, 1}-\mathbb{N}, \alpha=1, \ldots, A$. More precisely, $G(s ; \boldsymbol{s}, \lambda)$ has a pole of pure order $N_{\alpha, \beta}$ at $\lambda \in \lambda_{\alpha, 1}-\mathbb{N}$ if one let $\beta=$ $\max \left\{\beta^{\prime}: \lambda \leqslant_{1} \lambda_{\alpha, \beta^{\prime}}\right\}$. Moreover, in view of 1.14 in Lemma $1.3, G(s ; \boldsymbol{s}, \lambda)$ is of uniform moderate growth on vertical strips.

On the other hand, according to Corollary $1.11(1), \mathcal{M} v(1-s)$ uniformly rapidly decays on vertical strips.

Therefore, the product $G(s ; \boldsymbol{s}, \lambda) \mathcal{M} v(1-s)$ on the right hand side of 3.3$)$ is a meromorphic function in the space $\sum_{\alpha=1}^{A} \sum_{\beta=1}^{B_{\alpha}} \sum_{j=0}^{N_{\alpha \beta \beta}-1} \mathscr{M}_{\mathrm{sis}}^{\lambda_{\alpha \beta}, j}$. We conclude from Lemma 2.2 that (3.3) uniquely determines a function $\Upsilon$ in $\mathscr{S}_{\text {sis }}^{\lambda}\left(\mathbb{R}_{+}\right)$.

Q.E.D.

\subsubsection{The Bessel Function $J(x ; \boldsymbol{\varsigma}, \lambda)$.}

The Integral Kernel $J(x ; \boldsymbol{s}, \lambda)$ of $\mathcal{H}_{(\boldsymbol{\varsigma}, \lambda)}$. Suppose $v \in \mathscr{S}\left(\mathbb{R}_{+}\right)$. By the Mellin inversion, we have

$$
\Upsilon(x)=\frac{1}{2 \pi i} \int_{(\sigma)} G(s ; \boldsymbol{s}, \lambda) \mathcal{M} v(1-s) x^{-s} d s, \quad \sigma>\max \left\{\Re \mathfrak{R e} \lambda_{l}\right\} .
$$

It is an iterated double integral as below

$$
\Upsilon(x)=\frac{1}{2 \pi i} \int_{(\sigma)} \int_{0}^{\infty} v(y) y^{-s} d y \cdot G(s ; \boldsymbol{s}, \lambda) x^{-s} d s .
$$

We now shift the integral contour to $\mathcal{C}_{\lambda}$ defined in Definition 3.2 Using (1.14) in Lemma 1.3 , one shows that the above double integral becomes absolutely convergent after this contour shift. Therefore, on changing the order of integrals, one obtains

$$
\Upsilon(x)=\int_{0}^{\infty} v(y) J\left((x y)^{\frac{1}{n}} ; \boldsymbol{s}, \lambda\right) d y .
$$


Here $J(x ; \boldsymbol{s}, \lambda)$ is the Bessel function defined by the Mellin-Barnes type integral

$$
J(x ; \boldsymbol{\varsigma}, \lambda)=\frac{1}{2 \pi i} \int_{\mathrm{C}_{\lambda}} G(s ; \boldsymbol{\varsigma}, \lambda) x^{-n s} d s .
$$

Shifting the Index of $J(x ; \boldsymbol{\varsigma}, \lambda)$.

Lemma 3.5. Let $(\boldsymbol{s}, \lambda) \in\{+,-\}^{n} \times \mathbb{C}^{n}$ and $\lambda \in \mathbb{C}$. Recall that $\boldsymbol{e}^{n}$ denotes the $n$-tuple $(1, \ldots, 1)$. Then

$$
J\left(x ; \boldsymbol{\varsigma}, \lambda-\lambda \boldsymbol{e}^{n}\right)=x^{n \lambda} J(x ; \boldsymbol{s}, \lambda) .
$$

Regularity of $J(x ; \boldsymbol{\varsigma}, \lambda)$. We now show that $J(x ; \boldsymbol{\varsigma}, \lambda)$ admits an analytic continuation from $\mathbb{R}_{+}$onto $\mathbb{U}$. Consider the following Barnes type integral representation,

$$
J(\zeta ; \boldsymbol{s}, \lambda)=\frac{1}{2 \pi i} \int_{\mathcal{C}_{\lambda}^{\prime}} G(s ; \boldsymbol{s}, \lambda) \zeta^{-n s} d s, \quad \zeta=x e^{i \omega} \in \mathbb{U}, x \in \mathbb{R}_{+}, \omega \in \mathbb{R},
$$

with the integral contour given in Definition 3.3 We first rewrite $G(s, \pm)$ using Euler's reflection formula,

$$
G(s, \pm)=\frac{\pi e\left( \pm \frac{1}{4} s\right)}{\sin (\pi s) \Gamma(1-s)},
$$

Then Stirling's asymptotic formula 1.13 yields,

$$
G(-\rho+i t ; \boldsymbol{\varsigma}, \lambda) \ll_{\lambda, r} e^{n \rho} \rho^{-n\left(\rho+\frac{1}{2}\right)-\mathfrak{R e}|\lambda|},
$$

for all $-\rho+i t \notin \bigcup_{l=1}^{n} \bigcup_{\kappa \in \mathbb{N}} \mathbb{B}_{r}\left(\lambda_{l}-\kappa\right)$ satisfying $\rho \gg 1$ and $t \ll \max \left\{\left|\mathfrak{I} \mathfrak{m} \lambda_{l}\right|\right\}+1$. It follows that the contour integral in 3.8 converges absolutely and compactly in $\zeta$, and hence $J(\zeta ; \boldsymbol{\varsigma}, \lambda)$ is analytic in $\zeta$.

Moreover, given any bounded open subset of $\mathbb{C}^{n}$, one may fix a single contour $\mathrm{C}^{\prime}=\mathfrak{C}_{\lambda}^{\prime}$ for all $\lambda$ in this set and verify the uniform convergence of the integral in the $\lambda$ aspect. Then follows the analyticity of $J(\zeta ; \boldsymbol{\varsigma}, \lambda)$ with respect to $\lambda$.

Lemma 3.6. $J(x ; \boldsymbol{s}, \lambda)$ admits an analytic continuation $J(\zeta ; \boldsymbol{\varsigma}, \lambda)$ from $\mathbb{R}_{+}$onto $\mathbb{U}$. In particular, $J(x ; \boldsymbol{s}, \lambda)$ is a real analytic function of $x$ on $\mathbb{R}_{+}$. Moreover, $J(\zeta ; \boldsymbol{s}, \lambda)$ is an analytic function of $\lambda$ on $\mathbb{C}^{n}$.

Remark 3.7. In $\$ 10$ we shall show that $J(x ; \boldsymbol{\varsigma}, \lambda)$ satisfies a differential equation with analytic coefficients. It then follows from the theory of differential equations that $J(x ; \boldsymbol{\varsigma}, \lambda)$ has an analytic continuation. This alternative viewpoint will be taken in $\$ 11.2$

3.2. The Hankel Transforms $h_{(\mu, \delta)}, h_{(\mu, m)}$ and the Bessel Kernels $j_{(\mu, \delta)}, j_{(\mu, m)}$. Consider the ordered set $\left(\mathbb{C}, \leqslant_{2}\right)$ and define $\lambda_{\alpha, \beta}, B_{\alpha}, M_{\alpha, \beta}$ and $N_{\alpha, \beta}$ as in Definition 3.1 corresponding to $\lambda \in \mathbb{C}^{n}$. We define the following subspace of $\mathscr{S}_{\text {sis }}\left(\mathbb{R}_{+}\right)$

$$
\mathscr{T}_{\text {sis }}^{\lambda}\left(\mathbb{R}_{+}\right)=\sum_{\alpha=1}^{A} \sum_{\beta=1}^{B_{\alpha}} \sum_{j=0}^{N_{\alpha, \beta}-1} x^{-\lambda_{\alpha, \beta}}(\log x)^{j} \mathscr{S}_{0}\left(\overline{\mathbb{R}}_{+}\right) .
$$

3.2.1. The Definition of $h_{(\mu, \delta)}$. The following proposition provides the definition of the Hankel transform $\mathcal{f}_{(\mu, \delta)}$, which maps $\mathscr{T}_{\text {sis }}^{-\mu-\delta}\left(\mathbb{R}_{+}\right)$onto $\mathscr{T}_{\text {sis }}^{\mu-\delta}\left(\mathbb{R}_{+}\right)$bijectively.

Proposition 3.8. Let $(\boldsymbol{\mu}, \boldsymbol{\delta}) \in \mathbb{C}^{n} \times(\mathbb{Z} / 2 \mathbb{Z})^{n}$. Suppose $v \in \mathscr{T}_{\text {sis }}^{-\mu-\delta}\left(\mathbb{R}_{+}\right)$. Then there exists a unique function $\Upsilon \in \mathscr{T}_{\text {sis }}^{\mu-\delta}\left(\mathbb{R}_{+}\right)$satisfying the following identity,

$$
\mathcal{M} \Upsilon(s)=G_{(\mu, \delta)}(s) \mathcal{M} v(1-s) .
$$


We call $\Upsilon$ the Hankel transform of $v$ over $\mathbb{R}_{+}$of index $(\boldsymbol{\mu}, \boldsymbol{\delta})$ and write $h_{(\boldsymbol{\mu}, \delta)} v=\Upsilon$. Furthermore, we have the Hankel inversion formula

$$
h_{(\mu, \delta)} v=\Upsilon, \quad h_{(-\mu, \delta)} \Upsilon=(-)^{|\delta|} v .
$$

Proof. Recall the definition of $G_{(\mu, \delta)}$ given by $\left.1.3,1.4\right)$,

$$
G_{(\mu, \delta)}(s)=i^{|\delta|} \pi^{n\left(\frac{1}{2}-s\right)+|\mu|} \frac{\prod_{l=1}^{n} \Gamma\left(\frac{1}{2}\left(s-\mu_{l}+\delta_{l}\right)\right)}{\prod_{l=1}^{n} \Gamma\left(\frac{1}{2}\left(1-s+\mu_{l}+\delta_{l}\right)\right)},
$$

where $|\boldsymbol{\delta}|=\sum_{l} \delta_{l} \in \mathbb{N}$, with each $\delta_{l}$ viewed as a number in the set $\{0,1\} \subset \mathbb{N}$.

We write $\boldsymbol{\mu}^{ \pm}= \pm \boldsymbol{\mu}-\boldsymbol{\delta}$. Since $\mu_{l}^{+}+\mu_{l}^{-}=-2 \delta_{l} \in\{0,-2\}$, the partition $\left\{L_{\alpha}\right\}_{\alpha=1}^{A}$ of $\{1, \ldots, n\}$ and $B_{\alpha}$ in Definition 3.1 are the same for both $\mu^{+}$and $\boldsymbol{\mu}^{-}$. Let $\mu_{\alpha, \beta}^{ \pm}, M_{\alpha, \beta}^{ \pm}$and $N_{\alpha, \beta}^{ \pm}$ be the notations in Definition 3.1 corresponding to $\boldsymbol{\mu}^{ \pm}$. Then the Gamma quotient above may be rewritten as follows,

$$
\frac{\prod_{\alpha=1}^{A} \prod_{\beta=1}^{B_{\alpha}} \Gamma\left(\frac{1}{2}\left(s-\mu_{\alpha, \beta}^{+}\right)\right)^{M_{\alpha, \beta}^{+}}}{\prod_{\alpha=1}^{A} \prod_{\beta=1}^{B_{\alpha}} \Gamma\left(\frac{1}{2}\left(1-s-\mu_{\alpha, \beta}^{-}\right)\right)^{M_{\alpha, \beta}^{-}}} .
$$

Thus, at each point $\mu \in \mu_{\alpha, 1}^{+}-2 \mathbb{N}$ the product in the numerator contributes to $G_{(\mu, \delta)}(s)$ a pole of pure order $N_{\alpha, \beta}^{+}$, with $\beta=\max \left\{\beta^{\prime}: \mu \leqslant_{2} \mu_{\alpha, \beta^{\prime}}^{+}\right\}$, whereas at each point $\mu \in-\mu_{\alpha, 1}^{-}+2 \mathbb{N}+$ 1 the denominator contributes a zero of order $N_{\alpha, \beta}^{-}$, with $\beta=\max \left\{\beta^{\prime}: 1-\mu \leqslant_{2} \mu_{\alpha, \beta^{\prime}}^{-}\right\}$. Moreover, 1.15) in Lemma 1.3 implies that $G_{(\mu, \delta)}(s)$ is of uniform moderate growth on vertical strips.

On the other hand, according to Lemma 2.3, the Mellin transform $\mathcal{M} v$ lies in the space $\sum_{\alpha=1}^{A} \sum_{\beta=1}^{B_{\alpha}} \sum_{j=0}^{N_{\alpha, \beta}^{-}-1} \mathscr{N}_{\mathrm{sis}}^{\mu_{\alpha, \beta}^{-}, j}$. In particular, the poles of $\mathcal{M} v(1-s)$ are annihilated by the zeros contributed from the denominator of the Gamma quotient. Furthermore, $\mathcal{M} v(1-s)$ uniformly rapidly decays on vertical strips.

We conclude that the product $G_{(\mu, \delta)}(s) \mathcal{M} v(1-s)$ on the right hand side of 3.10) lies in the space $\sum_{\alpha=1}^{A} \sum_{\beta=1}^{B_{\alpha}} \sum_{j=0}^{N_{\alpha, \beta}^{+}-1} \mathscr{N}_{\text {sis }}^{\mu_{\alpha, \beta}^{+}, j}$, and hence $\Upsilon \in \mathscr{T}_{\text {sis }}^{\mu-\delta}\left(\mathbb{R}_{+}\right)$, with another application of Lemma 2.3 .

Finally, the Hankel inversion formula (3.11) is an immediate consequence of the functional relation 1.5 ) of gamma factors.

Q.E.D.

3.2.2. The Definition of $\kappa_{(\mu, m)}$. The following proposition provides the definition of the Hankel transform $\hat{h}_{(\boldsymbol{\mu}, \boldsymbol{m})}$, which maps $\mathscr{T}_{\text {sis }}^{-2 \boldsymbol{\mu}-\|\boldsymbol{m}\|}\left(\mathbb{R}_{+}\right)$onto $\mathscr{T}_{\text {sis }}^{2 \boldsymbol{\mu}-\|\boldsymbol{m}\|}\left(\mathbb{R}_{+}\right)$bijectively.

Proposition 3.9. Let $(\boldsymbol{\mu}, \boldsymbol{m}) \in \mathbb{C}^{n} \times \mathbb{Z}^{n}$. Suppose $v \in \mathscr{T}_{\text {sis }}^{-2 \boldsymbol{\mu}-\|\boldsymbol{m}\|}\left(\mathbb{R}_{+}\right)$. Then there exists a unique function $\Upsilon \in \mathscr{T}_{\text {sis }}^{2 \mu-\|m\|}\left(\mathbb{R}_{+}\right)$satisfying the following identity,

$$
\mathcal{M} \Upsilon(2 s)=G_{(\mu, m)}(s) \mathcal{M} v(2(1-s))
$$

We call $\Upsilon$ the Hankel transform of $v$ over $\mathbb{R}_{+}$of index $(\boldsymbol{\mu}, \boldsymbol{m})$ and write $h_{(\boldsymbol{\mu}, \boldsymbol{m})} v=\Upsilon$. Moreover, we have the Hankel inversion formula

$$
\hbar_{(\mu, m)} v=\Upsilon, \quad h_{(-\mu, m)} \Upsilon=(-)^{|m|} v .
$$

Proof. We first rewrite 3.12 as follows,

$$
\mathcal{M} \Upsilon(s)=G_{(\mu, m)}\left(\frac{s}{2}\right) \mathcal{M} v(2-s) .
$$


From 1.6, 1.7, we have

$$
G_{(\mu, \boldsymbol{m})}\left(\frac{s}{2}\right)=i^{|\|\boldsymbol{m}\||} \pi^{n(1-s)+2|\boldsymbol{\mu}|} \frac{\prod_{l=1}^{n} \Gamma\left(\frac{1}{2}\left(s-2 \mu_{l}+\left|m_{l}\right|\right)\right)}{\prod_{l=1}^{n} \Gamma\left(\frac{1}{2}\left(2-s+2 \mu_{l}+\left|m_{l}\right|\right)\right)},
$$

where $|\|\boldsymbol{m}\||=\sum_{l=1}^{n}\left|m_{l}\right|$ according to our notation. We can now proceed to apply the same arguments in the proof of Proposition 3.8. Here we use (1.16) and (1.8) instead of (1.15) and $(1.5)$ respectively.

Q.E.D.

\subsubsection{The Bessel Kernel $j_{(\mu, \delta)}$.}

The Definition of $j_{(\mu, \delta)}$. For $(\boldsymbol{\mu}, \boldsymbol{\delta}) \in \mathbb{C}^{n} \times(\mathbb{Z} / 2 \mathbb{Z})^{n}$, we define the Bessel kernel $j_{(\mu, \delta)}$ by the following Mellin-Barnes type integral,

$$
j_{(\mu, \delta)}(x)=\frac{1}{2 \pi i} \int_{\mathcal{C}_{(\mu, \delta)}} G_{(\mu, \delta)}(s) x^{-s} d s .
$$

It is clear that

$$
j_{\left(\mu-\mu e^{n}, \delta\right)}(x)=x^{\mu} j_{(\mu, \delta)}(x)
$$

In view of (1.9), we have

$$
j_{(\mu, \delta)}(x)=(2 \pi)^{|\boldsymbol{\mu}|} \sum_{\boldsymbol{s} \in\{+,-\}^{n}} \boldsymbol{\varsigma}^{\delta} J\left(2 \pi x^{\frac{1}{n}} ; \boldsymbol{\varsigma}, \boldsymbol{\mu}\right) .
$$

Regularity of $j_{(\mu, \delta)}$. It follows from 3.16 and Lemma 3.6 that $j_{(\mu, \delta)}(x)$ admits an analytic continuation $j_{(\mu, \delta)}(\zeta)$, which is also analytic with respect to $\mu$. Moreover, $j_{(\mu, \delta)}(\zeta)$ has the following Barnes type integral representation,

$$
j_{(\mu, \delta)}(\zeta)=\frac{1}{2 \pi i} \int_{\mathfrak{C}_{\mu-\delta}^{\prime}} G_{(\mu, \delta)}(s) \zeta^{-s} d s, \quad \zeta \in \mathbb{U} .
$$

To see the convergence, the following formula is required

$$
G_{\delta}(s)= \begin{cases}\frac{\pi(2 \pi)^{-s}}{\sin \left(\frac{1}{2} \pi s\right) \Gamma(1-s)}, & \text { if } \delta=0, \\ \frac{\pi i(2 \pi)^{-s}}{\cos \left(\frac{1}{2} \pi s\right) \Gamma(1-s)}, & \text { if } \delta=1 .\end{cases}
$$

The Integral Kernel of $h_{(\mu, \delta)}$. Suppose $v \in \mathscr{T}_{\text {sis }}^{-\mu-\delta}\left(\mathbb{R}_{+}\right)$. In order to proceed in the same way as in $\$ 3.1 .2$, one needs to assume that $(\boldsymbol{\mu}, \boldsymbol{\delta})$ satisfies the condition

$$
\min \left\{\Re \mathfrak{e} \mu_{l}+\delta_{l}\right\}+1>\max \left\{\Re \mathfrak{e} \mu_{l}-\delta_{l}\right\} .
$$

Then,

$$
\varsigma_{(\mu, \delta)} v(x)=\int_{0}^{\infty} v(y) j_{(\mu, \delta)}(x y) d y .
$$

Here, it is required for the convergence of the integral over $d y$ that the contour $\mathcal{C}_{(\mu, \delta)}$ in (3.14) is chosen to lie in the left half-plane $\left\{s: \mathfrak{R e} s<\min \left\{\mathfrak{R e} \mu_{l}+\delta_{l}\right\}+1\right\}$. According to Definition 3.2 this choice of $\mathcal{C}_{(\mu, \delta)}$ is permissible due to our assumption (3.19). However, if one assumes that $v \in \mathscr{S}\left(\mathbb{R}_{+}\right)$, then 3.20 remains valid without requiring the condition 3.19. 


\subsubsection{The Bessel Kernel $j_{(\mu, m)}$.}

The Definition of $j_{(\boldsymbol{\mu}, \boldsymbol{m})}$. For $(\boldsymbol{\mu}, \boldsymbol{m}) \in \mathbb{C}^{n} \times \mathbb{Z}^{n}$ define the Bessel kernel $j_{(\boldsymbol{\mu}, \boldsymbol{m})}$ by the following Mellin-Barnes type integral,

$$
j_{(\mu, \boldsymbol{m})}(x)=\frac{1}{2 \pi i} \int_{\mathcal{C}_{(\mu, \boldsymbol{m})}} G_{(\mu, \boldsymbol{m})}(s) x^{-2 s} d s .
$$

We have

$$
j_{\left(\boldsymbol{\mu}-\mu \boldsymbol{e}^{n}, \boldsymbol{m}\right)}(x)=x^{2 \mu} j_{(\boldsymbol{\mu}, \boldsymbol{m})}(x) .
$$

In view of Lemma 1.1 if $(\boldsymbol{\eta}, \boldsymbol{\delta}) \in \mathbb{C}^{2 n} \times(\mathbb{Z} / 2 \mathbb{Z})^{2 n}$ is related to $(\boldsymbol{\mu}, \boldsymbol{m}) \in \mathbb{C}^{n} \times \mathbb{Z}^{n}$ via either 1.11 or 1.12, then

$$
i^{n} j_{(\boldsymbol{\mu}, \boldsymbol{m})}(x)=j_{(\boldsymbol{\eta}, \boldsymbol{\delta})}\left(x^{2}\right) .
$$

Regularity of $j_{(\mu, m)}$. In view of 3.23 , the regularity of $j_{(\mu, m)}$ follows from that of $j_{(\boldsymbol{\eta}, \boldsymbol{\delta})}$. Alternatively, this may be seen from

$$
j_{(\mu, \boldsymbol{m})}(\zeta)=\frac{1}{2 \pi i} \int_{\mathcal{C}_{\mu-\frac{1}{2}\|\boldsymbol{m}\|}^{\prime}} G_{(\mu, \boldsymbol{m})}(s) \zeta^{-2 s} d s, \quad \zeta \in \mathbb{U} .
$$

To see the convergence, the following formula is required

$$
G_{m}(s)=\frac{\pi i^{|m|}(2 \pi)^{1-2 s}}{\sin \left(\pi\left(s+\frac{1}{2}|m|\right)\right) \Gamma\left(1-s-\frac{1}{2}|m|\right) \Gamma\left(1-s+\frac{1}{2}|m|\right)} .
$$

The Integral Kernel of $f_{(\boldsymbol{\mu}, \boldsymbol{m})}$. Suppose $v \in \mathscr{T}_{\text {sis }}^{-2 \boldsymbol{\mu}-\|\boldsymbol{m}\|}\left(\mathbb{R}_{+}\right)$. We assume that $(\boldsymbol{\mu}, \boldsymbol{m})$ satisfies the following condition

$$
\min \left\{\mathfrak{R e} \mu_{l}+\frac{1}{2}\left|m_{l}\right|\right\}+1>\max \left\{\mathfrak{R e} \mu_{l}-\frac{1}{2}\left|m_{l}\right|\right\} .
$$

Then

$$
f_{(\boldsymbol{\mu}, \boldsymbol{m})} v(x)=\int_{0}^{\infty} v(y) j_{(\boldsymbol{\mu}, \boldsymbol{m})}(x y) \cdot 2 y d y,
$$

It is required for convergence that the integral contour $\mathcal{C}_{(\mu, m)}$ in 3.21) lies in the left halfplane $\left\{s: \mathfrak{R e} s<\min \left\{\mathfrak{R e} \mu_{l}+\frac{1}{2}\left|m_{l}\right|\right\}+1\right\}$. This is however guaranteed by (3.26). Moreover, if one assumes that $v \in \mathscr{S}\left(\mathbb{R}_{+}\right)$, then 3.27$)$ holds true for any index $(\boldsymbol{\mu}, \boldsymbol{m})$.

Auxiliary Bounds for $j_{\left(\boldsymbol{\mu}, \boldsymbol{m}+m \boldsymbol{e}^{n}\right)}$.

Lemma 3.10. Let $(\boldsymbol{\mu}, \boldsymbol{m}) \in \mathbb{C}^{n} \times \mathbb{Z}^{n}$ and $m \in \mathbb{Z}$. Put

$$
\begin{aligned}
& A=n\left(\max \left\{\Re e \mu_{l}\right\}+\frac{1}{2} \max \left\{\left|m_{l}\right|\right\}-\frac{1}{2}\right)-\mathfrak{R e}|\boldsymbol{\mu}|+\frac{1}{2}|\|\boldsymbol{m}\||, \\
& B_{+}=-2 \min \left\{\Re \mathfrak{e} \mu_{l}\right\}+\max \left\{\left|m_{l}\right|\right\}+\max \left\{\frac{1}{n}-\frac{1}{2}, 0\right\}, \\
& B_{-}=-2 \max \left\{\Re \mathrm{Re} \mu_{l}\right\}-\max \left\{\left|m_{l}\right|\right\} .
\end{aligned}
$$

Fix $\epsilon>0$. Denote by $\boldsymbol{e}^{n}$ the $n$-tuple $(1, \ldots, 1)$. We have the following estimate

$$
j_{\left(\mu, \boldsymbol{m}+m \boldsymbol{e}^{n}\right)}(x) \ll_{(\boldsymbol{\mu}, \boldsymbol{m}), \epsilon, n}\left(\frac{2 \pi e x^{\frac{1}{n}}}{|m|+1}\right)^{n|m|}(|m|+1)^{A+n \epsilon} \max \left\{x^{B_{+}+2 \epsilon}, x^{B_{-}-2 \epsilon}\right\} .
$$

Proof. Let

$$
\begin{aligned}
\rho_{m} & =\max \left\{\Re \mathfrak{e} \mu_{l}-\frac{1}{2}\left|m_{l}+m\right|\right\} \\
\sigma_{m} & =\min \left\{\frac{1}{2}+\frac{1}{n}\left(\Re \mathfrak{R}|\boldsymbol{\mu}|-\frac{1}{2}\left|\left\|\boldsymbol{m}+m \boldsymbol{e}^{n}\right\|\right|-1\right), \rho_{m}\right\} .
\end{aligned}
$$


Choose the contour $\mathcal{C}_{m}=\mathcal{C}_{\left(\mu, m+m e^{n}\right)}$ (see Definition 3.2p such that

- if $s \in \mathcal{C}_{m}$ and $\mathfrak{I m} s$ is sufficiently large, then $\Re \mathrm{e} s=\sigma_{m}-\epsilon$, and

- $\mathcal{C}_{m}$ lies in the vertical strip $\mathbb{S}\left[\sigma_{m}-\epsilon, \rho_{m}+\epsilon\right]$.

We first assume that $|m|$ is large enough so that

$$
n\left(\rho_{m}+\epsilon-\frac{1}{2}\right)-\mathfrak{R e}|\boldsymbol{\mu}|-\frac{1}{2}||\left|\boldsymbol{m}+m \boldsymbol{e}^{n} \|\right|<0 .
$$

For the sake of brevity, we write $y=(2 \pi)^{n} x$. We first bound $\left|j_{\left(\mu, m+m e^{n}\right)}(x)\right|$ by

$$
(2 \pi)^{n+\Re \mathfrak{e}|\mu|} \int_{\mathfrak{C}_{m}} y^{-2 \Re \mathrm{R} s} \prod_{l=1}^{n}\left|\frac{\Gamma\left(s-\mu_{l}+\frac{1}{2}\left|m_{l}+m\right|\right)}{\Gamma\left(1-s+\mu_{l}+\frac{1}{2}\left|m_{l}+m\right|\right)}\right| d s \mid .
$$

With the observations that for $s \in \mathcal{C}_{m}$

- Re $s \in\left[\sigma_{m}-\epsilon, \rho_{m}+\epsilon\right]$,

- $\mid$ Re $s-\mu_{l}+\frac{1}{2}\left|m_{l}+m\right| \mid \ll_{(\mu, m)} 1$,

- $\left|\left(1-\Re \mathrm{e} s+\mu_{l}+\frac{1}{2}\left|m_{l}+m\right|\right)-\right| m|| \ll_{(\mu, m)} 1$,

in conjunction with Stirling's asymptotic formula 1.13 , we have the following estimate

$$
\begin{aligned}
& j_{\left(\mu, \boldsymbol{m}+m e^{n}\right)}(x) \ll_{(\boldsymbol{\mu}, \boldsymbol{m}), n, \epsilon} \max \left\{y^{-2 \sigma_{m}+2 \epsilon}, y^{-2 \rho_{m}-2 \epsilon}\right\} \\
& \int_{\mathfrak{C}_{m}} \frac{(|\mathfrak{I m} s|+1)^{n\left(\mathfrak{R e} s-\frac{1}{2}\right)-\Re \mathfrak{e}|\boldsymbol{\mu}|+\frac{1}{2}|| \boldsymbol{m}+m \boldsymbol{e}^{n} \| \mid}}{e^{-n|m|}\left(\sqrt{(\mathfrak{J} \mathfrak{m} s)^{2}+m^{2}}+1\right)^{n\left(\frac{1}{2}-\mathfrak{R e} s\right)+\Re \mathfrak{e}|\boldsymbol{\mu}|+\frac{1}{2}|| \boldsymbol{m}+m \boldsymbol{e}^{n} \| \mid}}|d s| \\
& \leqslant \max \left\{y^{-2 \sigma_{m}+2 \epsilon}, y^{-2 \rho_{m}-2 \epsilon}\right\} e^{n|m|}(|m|+1)^{n\left(\rho_{m}+\epsilon-\frac{1}{2}\right)-\Re \mathfrak{e}|\boldsymbol{\mu}|-\frac{1}{2}|| \boldsymbol{m}+m \boldsymbol{e}^{n} \| \mid} \\
& \int_{\mathfrak{C}_{m}}(|\mathfrak{I m} s|+1)^{n\left(\Re e s-\frac{1}{2}\right)-\Re \mathfrak{R}|\boldsymbol{\mu}|+\frac{1}{2}|| \boldsymbol{m}+m e^{n} \| \mid}|d s| .
\end{aligned}
$$

For $s \in \mathcal{C}_{m}$, we have $\mathfrak{R e} s=\sigma_{m}-\epsilon$ if $\mathfrak{I m} s$ is sufficiently large, and our choice of $\sigma_{m}$ implies $n\left(\sigma_{m}-\epsilon-\frac{1}{2}\right)-\mathfrak{R e}|\boldsymbol{\mu}|+\frac{1}{2}\left|\left\|\boldsymbol{m}+m \boldsymbol{e}^{n}\right\|\right| \leqslant-1-n \epsilon$, then it follows that the above integral converges and is of size $O_{(\mu, m), \epsilon, n}(1)$.

Finally, note that both $-2 \sigma_{m}+2 \epsilon$ and $-2 \rho_{m}-2 \epsilon$ are close to $|m|$, whereas the exponent of $(|m|+1)$, that is $n\left(\rho_{m}+\epsilon-\frac{1}{2}\right)-\Re \mathfrak{e}|\boldsymbol{\mu}|-\frac{1}{2}\left|\left\|\boldsymbol{m}+m \boldsymbol{e}^{n}\right\|\right|$, is close to $-n|m|$. Thus the following bounds yield 3.28 ,

$$
\begin{aligned}
& |m|+B_{-} \leqslant-2 \rho_{m} \leqslant-2 \sigma_{m} \leqslant|m|+B_{+}, \\
& n\left(\rho_{m}-\frac{1}{2}\right)-\mathfrak{R e}|\boldsymbol{\mu}|-\frac{1}{2}|| \boldsymbol{m}+m \boldsymbol{e}^{n} \||\leqslant-n| m \mid+A .
\end{aligned}
$$

When $|m|$ is small, we have the following estimate that also implies (3.28),

$$
j_{\left(\boldsymbol{\mu}, \boldsymbol{m}+m e^{n}\right)}(x) \ll_{(\boldsymbol{\mu}, \boldsymbol{m}), \epsilon, n} \max \left\{y^{-2 \sigma_{m}+2 \epsilon}, y^{-2 \rho_{m}-2 \epsilon}\right\} e^{n|m|} .
$$

Q.E.D.

Using the formula (3.25) of $G_{m}(s)$ instead of (1.6) and the Barnes type integral representation 3.24 for $j_{\left(\mu, m+m e^{n}\right)}(\zeta)$ instead of the Mellin-Barnes type integral representation (3.21) for $j_{\left(\mu, m+m e^{n}\right)}(x)$, similar arguments in the proof of Lemma 3.10 imply the following lemma.

Lemma 3.11. Let $(\boldsymbol{\mu}, \boldsymbol{m}) \in \mathbb{C}^{n} \times \mathbb{Z}^{n}$ and $m \in \mathbb{Z}$. Put

$$
\begin{aligned}
& A=n\left(\max \left\{\mathfrak{R e} \mu_{l}\right\}+\frac{1}{2} \max \left\{\left|m_{l}\right|\right\}-\frac{1}{2}\right)-\mathfrak{R e}|\boldsymbol{\mu}|+\frac{1}{2}|\|\boldsymbol{m}\||, \\
& B=-2 \max \left\{\mathfrak{R e} \mu_{l}\right\}-\max \left\{\left|m_{l}\right|\right\}, \quad C=2 \max \left\{\left|\mathfrak{I m} \mu_{l}\right|\right\} .
\end{aligned}
$$


Fix $X>0$ and $\epsilon>0$. Then

$$
j_{\left(\boldsymbol{\mu}, \boldsymbol{m}+m \boldsymbol{e}^{n}\right)}\left(x e^{i \omega}\right) \ll_{(\boldsymbol{\mu}, \boldsymbol{m}), X, \epsilon, n}\left(\frac{2 \pi e x^{\frac{1}{n}}}{|m|+1}\right)^{n|m|}(|m|+1)^{A+n \epsilon} x^{B+2 \epsilon} e^{|\omega|(C+2 \epsilon)}
$$

for all $x<X$.

\subsection{The Hankel Transform $\mathcal{H}_{(\mu, \delta)}$ and the Bessel Kernel $J_{(\mu, \delta)}$.}

3.3.1. The Definition of $\mathcal{H}_{(\mu, \delta)}$. Consider the ordered set $(\mathbb{C} \times \mathbb{Z} / 2 \mathbb{Z}, \preccurlyeq)$ and define $\left(\mu_{\alpha, \beta}, \delta_{\alpha, \beta}\right)=(\mu, \delta)_{\alpha, \beta}, B_{\alpha}, M_{\alpha, \beta}$ and $N_{\alpha, \beta}$ as in Definition 3.1 corresponding to $(\boldsymbol{\mu}, \boldsymbol{\delta}) \in$ $(\mathbb{C} \times \mathbb{Z} / 2 \mathbb{Z})^{n}$. We define the following subspaces of $\mathscr{S}_{\text {sis }}\left(\mathbb{R}^{\times}\right)$,

$$
\begin{aligned}
& \mathscr{S}_{\mathrm{sis}}^{(\boldsymbol{\mu}, \boldsymbol{\delta}), \delta}\left(\mathbb{R}^{\times}\right)=\sum_{\alpha=1}^{A} \sum_{\beta=1}^{B_{\alpha}} \sum_{j=0}^{N_{\alpha, \beta}-1} \operatorname{sgn}(x)^{\delta_{\alpha, \beta}}|x|^{-\mu_{\alpha, \beta}}(\log |x|)^{j} \mathscr{S}_{\delta_{\alpha, \beta}+\delta}(\mathbb{R}) . \\
& \mathscr{S}_{\text {sis }}^{(\mu, \delta)}\left(\mathbb{R}^{\times}\right)=\mathscr{S}_{\text {sis }}^{(\mu, \delta), 0}\left(\mathbb{R}^{\times}\right) \oplus \mathscr{S}_{\text {sis }}^{(\mu, \delta), 1}\left(\mathbb{R}^{\times}\right) \\
& =\sum_{\alpha=1}^{A} \sum_{\beta=1}^{B_{\alpha}} \sum_{j=0}^{N_{\alpha, \beta}-1} \operatorname{sgn}(x)^{\delta_{\alpha, \beta}}|x|^{-\mu_{\alpha, \beta}}(\log |x|)^{j} \mathscr{S}(\mathbb{R}) .
\end{aligned}
$$

From the definition of $\mathscr{T}_{\text {sis }}^{\lambda}\left(\mathbb{R}_{+}\right)$in 3.9, , together with $\mathscr{S}_{\delta}(\mathbb{R})=\operatorname{sgn}(x)^{\delta} \mathscr{S}_{\delta}\left(\overline{\mathbb{R}}_{+}\right)$and $\mathscr{S}_{\delta}\left(\overline{\mathbb{R}}_{+}\right)=x^{\delta} \mathscr{S}_{0}\left(\overline{\mathbb{R}}_{+}\right)$, we have

$$
\mathscr{S}_{\text {sis }}^{(\boldsymbol{\mu}, \boldsymbol{\delta}), \delta}\left(\mathbb{R}^{\times}\right)=\operatorname{sgn}(x)^{\delta} \mathscr{T}_{\text {sis }}^{\boldsymbol{\mu}-\left(\boldsymbol{\delta}+\delta \boldsymbol{e}^{n}\right)}\left(\mathbb{R}_{+}\right) .
$$

The following theorem gives the definition of the Hankel transform $\mathcal{H}_{(\mu, \delta)}$, which $\operatorname{maps} \mathscr{S}_{\text {sis }}^{(-\boldsymbol{\mu}, \boldsymbol{\delta})}\left(\mathbb{R}^{\times}\right)$onto $\mathscr{S}_{\text {sis }}^{(\boldsymbol{\mu} \boldsymbol{\delta})}\left(\mathbb{R}^{\times}\right)$bijectively.

Theorem 3.12. Let $(\boldsymbol{\mu}, \boldsymbol{\delta}) \in \mathbb{C}^{n} \times(\mathbb{Z} / 2 \mathbb{Z})^{n}$. Suppose $v \in \mathscr{S}_{\text {sis }}^{(-\boldsymbol{\mu}, \boldsymbol{\delta})}\left(\mathbb{R}^{\times}\right)$. Then there exists a unique function $\Upsilon \in \mathscr{S}_{\text {sis }}^{(\mu, \delta)}\left(\mathbb{R}^{\times}\right)$satisfying the following two identities,

$$
\mathcal{M}_{\delta} \Upsilon(s)=G_{\left(\mu, \delta+\delta e^{n}\right)}(s) \mathcal{M}_{\delta} v(1-s), \quad \delta \in \mathbb{Z} / 2 \mathbb{Z} .
$$

We call $\Upsilon$ the Hankel transform of $v$ over $\mathbb{R}^{\times}$of index $(\boldsymbol{\mu}, \boldsymbol{\delta})$ and write $\mathcal{H}_{(\boldsymbol{\mu}, \boldsymbol{\delta})} v=\Upsilon$. Moreover, we have the Hankel inversion formula

$$
\mathcal{H}_{(\mu, \delta)} v(x)=\Upsilon(x), \quad \mathcal{H}_{(-\mu, \delta)} \Upsilon(x)=(-)^{|\delta|} v\left((-)^{n} x\right)
$$

Proof. Recall that

$$
\mathcal{M}{ }_{\delta} v(s)=2 \mathcal{M} v_{\delta}(s) \text {. }
$$

In view of (3.31), one has $v_{\delta} \in \mathscr{T}_{\text {sis }}^{-\boldsymbol{\mu}-\left(\boldsymbol{\delta}+\delta \boldsymbol{e}^{n}\right)}\left(\mathbb{R}_{+}\right)$. Applying Proposition 3.8, there is a unique function $\Upsilon_{\delta} \in \mathscr{T}_{\text {sis }}^{\boldsymbol{\mu}-\left(\boldsymbol{\delta}+\delta \boldsymbol{e}^{n}\right)}\left(\mathbb{R}_{+}\right)$satisfying

$$
\mathcal{M} \Upsilon_{\delta}(s)=G_{\left(\mu, \delta+\delta e^{n}\right)}(s) \mathcal{M} v_{\delta}(1-s)
$$

According to 3.31), $\Upsilon(x)=\Upsilon_{0}(|x|)+\operatorname{sgn}(x) \Upsilon_{1}(|x|)$ lies in $\mathscr{S}_{\text {sis }}^{(\mu, \delta), 0}\left(\mathbb{R}^{\times}\right) \oplus \mathscr{S}_{\text {sis }}^{(\mu, \delta), 1}\left(\mathbb{R}^{\times}\right)=$ $\mathscr{S}_{\text {sis }}^{(\mu, \delta)}\left(\mathbb{R}^{\times}\right)$. Clearly, $\Upsilon$ satisfies (3.32). Moreover, 3.33) follows immediately from 3.11) in Proposition 3.8 .

Q.E.D.

Corollary 3.13. Let $(\boldsymbol{\mu}, \boldsymbol{\delta}) \in \mathbb{C}^{n} \times(\mathbb{Z} / 2 \mathbb{Z})^{n}$ and $\delta \in \mathbb{Z} / 2 \mathbb{Z}$. Suppose that $\varphi \in$ $\mathscr{T}_{\text {sis }}^{-\mu-\left(\delta+\delta \boldsymbol{e}^{n}\right)}\left(\mathbb{R}_{+}\right)$and $v(x)=\operatorname{sgn}(x)^{\delta} \varphi(|x|)$. Then

$$
\mathcal{H}_{(\mu, \delta)} v( \pm x)=( \pm)^{\delta} h_{\left(\mu, \delta+\delta e^{n}\right)} \varphi(x), \quad x \in \mathbb{R}_{+} .
$$


3.3.2. The Bessel Kernel $J_{(\boldsymbol{\mu}, \boldsymbol{\delta})}$. Let $(\boldsymbol{\mu}, \boldsymbol{\delta}) \in \mathbb{C}^{n} \times(\mathbb{Z} / 2 \mathbb{Z})^{n}$. We define

$$
J_{(\boldsymbol{\mu}, \boldsymbol{\delta})}( \pm x)=\frac{1}{2} \sum_{\delta \in \mathbb{Z} / 2 \mathbb{Z}}( \pm)^{\delta} j_{\left(\boldsymbol{\mu}, \boldsymbol{\delta}+\delta \boldsymbol{e}^{n}\right)}(x), \quad x \in \mathbb{R}_{+},
$$

or equivalently,

$$
J_{(\mu, \delta)}(x)=\frac{1}{2} \sum_{\delta \in \mathbb{Z} / 2 \mathbb{Z}} \operatorname{sgn}(x)^{\delta} j_{\left(\mu, \delta+\delta \boldsymbol{e}^{n}\right)}(|x|), \quad x \in \mathbb{R}^{\times} .
$$

Some properties of $J_{(\mu, \delta)}$ are summarized as below.

Proposition 3.14. Let $(\boldsymbol{\mu}, \boldsymbol{\delta}) \in \mathbb{C}^{n} \times(\mathbb{Z} / 2 \mathbb{Z})^{n}$.

(1). Let $(\mu, \delta) \in \mathbb{C} \times \mathbb{Z} / 2 \mathbb{Z}$. We have

$$
J_{\left(\boldsymbol{\mu}-\mu \boldsymbol{e}^{n}, \boldsymbol{\delta}-\delta \boldsymbol{e}^{n}\right)}(x)=\operatorname{sgn}(x)^{\delta}|x|^{\mu} J_{(\boldsymbol{\mu}, \boldsymbol{\delta})}(x) .
$$

(2). $J_{(\mu, \delta)}(x)$ is a real analytic function of $x$ on $\mathbb{R}^{\times}$as well as an analytic function of $\boldsymbol{\mu}$ on $\mathbb{C}^{n}$.

(3). Assume that $\boldsymbol{\mu}$ satisfies the condition

$$
\min \left\{\mathfrak{R e} \mu_{l}\right\}+1>\max \left\{\mathfrak{R e} \mu_{l}\right\} .
$$

Then for $v \in \mathscr{S}_{\text {sis }}^{(-\mu, \delta)}\left(\mathbb{R}^{\times}\right)$

$$
\mathcal{H}_{(\mu, \delta)} v(x)=\int_{\mathbb{R}^{\times}} v(y) J_{(\mu, \delta)}(x y) d y .
$$

Moreover, if $v \in \mathscr{S}\left(\mathbb{R}^{\times}\right)$, then 3.37 remains true for any index $\boldsymbol{\mu} \in \mathbb{C}^{n}$.

\subsection{The Hankel Transform $\mathcal{H}_{(\mu, m)}$ and the Bessel Kernel $J_{(\mu, m)}$.}

3.4.1. The Definition of $\mathcal{H}_{(\mu, m)}$. Consider now the ordered set $(\mathbb{C} \times \mathbb{Z}, \preccurlyeq)$ and define $\left(2 \mu_{\alpha, \beta}, m_{\alpha, \beta}\right)=(2 \mu, m)_{\alpha, \beta}, B_{\alpha}, M_{\alpha, \beta}$ and $N_{\alpha, \beta}$ as in Definition 3.1 corresponding to $(2 \boldsymbol{\mu}, \boldsymbol{m}) \in(\mathbb{C} \times \mathbb{Z})^{n}$. We define the following subspace of $\mathscr{S}_{\text {sis }}\left(\mathbb{C}^{\times}\right)$,

$$
\mathscr{S}_{\mathrm{sis}}^{(\boldsymbol{\mu}, \boldsymbol{m})}\left(\mathbb{C}^{\times}\right)=\sum_{\alpha=1}^{A} \sum_{\beta=1}^{B_{\alpha}} \sum_{j=0}^{N_{\alpha, \beta}-1}[z]^{-m_{\alpha, \beta}}\|z\|^{-\mu_{\alpha, \beta}}(\log |z|)^{j} \mathscr{S}(\mathbb{C}) .
$$

The projection via the $m$-th Fourier coefficient maps $\mathscr{S}_{\text {sis }}^{(\mu, m)}\left(\mathbb{C}^{\times}\right)$onto the space

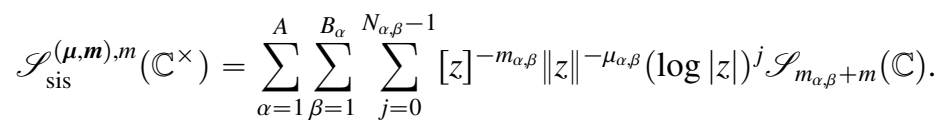

From the definition of $\mathscr{T}_{\text {sis }}^{\lambda}\left(\mathbb{R}_{+}\right)$in $\left[3.9\right.$, along with $\mathscr{S}_{m}(\mathbb{C})=[z]^{m} \mathscr{S}_{m}\left(\overline{\mathbb{R}}_{+}\right)$and $\mathscr{S}_{m}\left(\overline{\mathbb{R}}_{+}\right)=$ $x^{|m|} \mathscr{S}_{0}\left(\overline{\mathbb{R}}_{+}\right)$, we have

$$
\mathscr{S}_{\text {sis }}^{(\boldsymbol{\mu}, \boldsymbol{m}), m}\left(\mathbb{C}^{\times}\right)=[z]^{m} \mathscr{T}_{\text {sis }}^{2 \boldsymbol{\mu}-\left\|\boldsymbol{m}+m \boldsymbol{e}^{n}\right\|}\left(\mathbb{R}_{+}\right) .
$$

The following theorem gives the definition of the Hankel transform $\mathcal{H}_{(\mu, m)}$, which maps $\mathscr{S}_{\text {sis }}^{(-\boldsymbol{\mu},-\boldsymbol{m})}\left(\mathbb{C}^{\times}\right)$onto $\mathscr{S}_{\text {sis }}^{(\boldsymbol{\mu}, \boldsymbol{m})}\left(\mathbb{C}^{\times}\right)$bijectively.

Theorem 3.15. Let $(\boldsymbol{\mu}, \boldsymbol{m}) \in \mathbb{C}^{n} \times \mathbb{Z}^{n}$. Suppose $v \in \mathscr{S}_{\text {sis }}^{(-\boldsymbol{\mu},-\boldsymbol{m})}\left(\mathbb{C}^{\times}\right)$. Then there exists a unique function $\Upsilon \in \mathscr{S}_{\mathrm{sis}}^{(\mu, m)}\left(\mathbb{C}^{\times}\right)$satisfying the following sequence of identities,

$$
\mathcal{M}_{-m} \Upsilon(2 s)=G_{\left(\boldsymbol{\mu}, \boldsymbol{m}+m \boldsymbol{e}^{n}\right)}(s) \mathcal{M}_{m} v(2(1-s)), \quad m \in \mathbb{Z}
$$


We call $\Upsilon$ the Hankel transform of $v$ over $\mathbb{C}^{\times}$of index $(\boldsymbol{\mu}, \boldsymbol{m})$ and write $\mathcal{H}_{(\boldsymbol{\mu}, \boldsymbol{m})} v=\Upsilon$. Moreover, we have the Hankel inversion formula

$$
\mathcal{H}_{(\boldsymbol{\mu}, \boldsymbol{m})} v(z)=\Upsilon(z), \quad \mathcal{H}_{(-\boldsymbol{\mu},-\boldsymbol{m})} \Upsilon(z)=(-)^{|\boldsymbol{m}|} v\left((-)^{n} z\right) .
$$

Proof. Recall that

$$
\mathcal{M}_{m} v(s)=4 \pi \mathcal{M} v_{-m}(s) .
$$

In view of 3.40, we have $v_{-m} \in \mathscr{T}_{\text {sis }}^{-2 \mu-\left\|\boldsymbol{m}+m e^{n}\right\|}\left(\mathbb{R}_{+}\right)$. Applying Proposition 3.9. we infer that there is a unique function $\Upsilon_{m} \in \mathscr{T}_{\text {sis }}^{2 \mu-\left\|m+m e^{n}\right\|}\left(\mathbb{R}_{+}\right)$satisfying

$$
\mathcal{M} \Upsilon \Upsilon_{m}(2 s)=G_{\left(\mu, m+m e^{n}\right)}(s) \mathcal{M} v_{-m}(2(1-s)) .
$$

According to Lemma 2.8, in order to show that the Fourier series $\Upsilon\left(x e^{i \phi}\right)=\sum \Upsilon_{m}(x) e^{i m \phi}$ lies in $\mathscr{S}_{\text {sis }}^{(\boldsymbol{\mu}, \boldsymbol{m})}\left(\mathbb{C}^{\times}\right)$, it suffices to verify that $G_{\left(\boldsymbol{\mu}, \boldsymbol{m}+m e^{n}\right)}(s) \mathcal{M} v_{-m}(2(1-s))$ rapidly decays with respect to $m$, uniformly on vertical strips. This however follows from the uniform rapid decay of $\mathcal{N} v_{-m}(2(1-s))$ along with the uniform moderate growth of $G_{\left(\mu, m+m e^{n}\right)}(s)$ ( 1.16 in Lemma 1.3 in the $m$ aspect on vertical strips.

Finally, (3.13) in Proposition 3.9 implies (3.42).

Q.E.D.

Corollary 3.16. Let $(\boldsymbol{\mu}, \boldsymbol{m}) \in \mathbb{C}^{n} \times \mathbb{Z}^{n}$ and $m \in \mathbb{Z}$. Suppose $\varphi \in \mathscr{T}_{\text {sis }}^{-2 \boldsymbol{\mu}-\left\|\boldsymbol{m}+m e^{n}\right\|}\left(\mathbb{R}_{+}\right)$ and $v(z)=[z]^{-m} \varphi(|z|)$. Then

$$
\mathcal{H}_{(\mu, \boldsymbol{m})} v\left(x e^{i \phi}\right)=e^{i m \phi} \hbar_{\left(\mu, \boldsymbol{m}+m e^{n}\right)} \varphi(x), \quad x \in \mathbb{R}_{+}, \phi \in \mathbb{R} / 2 \pi \mathbb{Z} .
$$

3.4.2. The Bessel Kernel $J_{(\mu, \boldsymbol{m})}$. For $(\boldsymbol{\mu}, \boldsymbol{m}) \in \mathbb{C}^{n} \times \mathbb{Z}^{n}$, we define

$$
J_{(\mu, \boldsymbol{m})}\left(x e^{i \phi}\right)=\frac{1}{2 \pi} \sum_{m \in \mathbb{Z}} j_{\left(\mu, \boldsymbol{m}+m \boldsymbol{e}^{n}\right)}(x) e^{i m \phi},
$$

or equivalently,

$$
J_{(\mu, m)}(z)=\frac{1}{2 \pi} \sum_{m \in \mathbb{Z}} j_{\left(\mu, m+m e^{n}\right)}(|z|)[z]^{m} .
$$

Lemma 3.10 secures the absolute convergence of this series.

Proposition 3.17. Let $(\boldsymbol{\mu}, \boldsymbol{m}) \in \mathbb{C}^{n} \times \mathbb{Z}^{n}$.

(1). Let $(\mu, m) \in \mathbb{C} \times \mathbb{Z}$. We have

$$
J_{\left(\boldsymbol{\mu}-\mu \boldsymbol{e}^{n}, \boldsymbol{m}-m \boldsymbol{e}^{n}\right)}(z)=[z]^{m}\|z\|^{\mu} J_{(\boldsymbol{\mu}, \boldsymbol{m})}(z) .
$$

(2). $J_{(\mu, m)}(z)$ is a real analytic function of $z$ on $\mathbb{C}^{\times}$as well as an analytic function of $\mu$ on $\mathbb{C}^{n}$.

(3). Assume that $\boldsymbol{\mu}$ satisfies the following condition

$$
\min \left\{\mathfrak{R e} \mu_{l}\right\}+1>\max \left\{\mathfrak{R e} \mu_{l}\right\} .
$$

Suppose $v \in \mathscr{S}_{\text {sis }}^{(-\mu,-m)}\left(\mathbb{C}^{\times}\right)$. Then

$$
\Upsilon\left(x e^{i \phi}\right)=\int_{0}^{\infty} \int_{0}^{2 \pi} v\left(y e^{i \theta}\right) J_{(\mu, \boldsymbol{m})}\left(x e^{i \phi} y e^{i \theta}\right) \cdot 2 y d \theta d y,
$$

or equivalently,

$$
\Upsilon(z)=\int_{\mathbb{C}^{\times}} v(u) J_{(\mu, m)}(z u) d u .
$$

Moreover, 3.46 and 3.47) still hold true for any index $\boldsymbol{\mu} \in \mathbb{C}$ if $v \in \mathscr{S}\left(\mathbb{C}^{\times}\right)$. 
Proof. (1). This is clear.

(2). In (3.43), with abuse of notation, we view $x$ and $\phi$ as complex variables on $\mathbb{U}$ and $\mathbb{C} / 2 \pi \mathbb{Z}$ respectively, $j_{\left(\mu, \boldsymbol{m}+m \boldsymbol{e}^{n}\right)}(x)$ and $e^{i m \phi}$ as analytic functions. Then Lemma 3.11 implies that the series in 3.43 is absolutely convergent, compactly with respect to both $x$ and $\phi$, and therefore $J_{(\mu, m)}\left(x e^{i \phi}\right)$ is an analytic function of $x$ and $\phi$. In particular, $J_{(\mu, m)}(z)$ is a real analytic function of $z$ on $\mathbb{C}^{\times}$.

Moreover, in Lemma 3.10, we may allow $\boldsymbol{\mu}$ to vary in an $\epsilon$-ball in $\mathbb{C}^{n}$ and choose the implied constant in the estimate to be uniformly bounded with respect to $\boldsymbol{\mu}$. This implies that the series in 3.43 is convergent compactly in the $\boldsymbol{\mu}$ aspect. Therefore, $J_{(\mu, m)}(z)$ is an analytic function of $\boldsymbol{\mu}$ on $\mathbb{C}^{n}$.

(3). It follows from 3.40 that $v_{-m} \in \mathscr{T}_{\text {sis }}^{-2 \boldsymbol{\mu}-\left\|\boldsymbol{m}+m \boldsymbol{e}^{n}\right\|}\left(\mathbb{R}_{+}\right)$. Moreover, one observes that $\left(\boldsymbol{\mu}, \boldsymbol{m}+\boldsymbol{m} \boldsymbol{e}^{n}\right)$ satisfies the condition (3.26) due to (3.45). Therefore, in conjunction with Proposition 3.9. 3.27) implies

$$
\Upsilon_{m}(x)=2 \int_{0}^{\infty} v_{-m}(y) j_{\left(\mu, \boldsymbol{m}+m \boldsymbol{e}^{n}\right)}(x y) y d y .
$$

Hence

$$
\Upsilon\left(x e^{i \phi}\right)=\sum_{m \in \mathbb{Z}} \Upsilon_{m}(x) e^{i m \phi}=\sum_{m \in \mathbb{Z}} \frac{1}{\pi} \int_{0}^{\infty} \int_{0}^{2 \pi} v\left(y e^{i \theta}\right) j_{\left(\mu, \boldsymbol{m}+m e^{n}\right)}(x y) e^{i m(\phi+\theta)} y d \theta d y
$$

The estimate of $j_{\left(\mu, m+m e^{n}\right)}$ in Lemma 3.10 implies that the above series of integrals converges absolutely. On interchanging the order of summation and integration, one obtains 3.46) in view of the definition of $J_{(\mu, m)}$ in 3.43.

Note that in the case $v \in \mathscr{S}\left(\mathbb{C}^{\times}\right)$, one has $v_{-m} \in \mathscr{S}\left(\mathbb{R}_{+}\right)$, and therefore 3.27) can be applied unconditionally.

Q.E.D.

\subsection{Concluding Remarks.}

3.5.1. Connection Formulae. From 3.16, 3.34) or 3.35, we deduce the connection formula

$$
J_{(\boldsymbol{\mu}, \boldsymbol{\delta})}( \pm x)=(2 \pi)^{|\boldsymbol{\mu}|} \sum_{|\boldsymbol{s}|= \pm} \boldsymbol{\varsigma}^{\delta} J\left(2 \pi x^{\frac{1}{n}} ; \boldsymbol{\varsigma}, \boldsymbol{\mu}\right)
$$

This will enable us to reduce the study of $J_{(\mu, \delta)}(x)$ to that of $J(x ; \boldsymbol{s}, \boldsymbol{\lambda})$.

From the various connection formulae $3.16,3.23,3.43,3.44$ which have been derived so far, one can also connect the Bessel kernel $J_{(\mu, \boldsymbol{m})}(z)$ to the Bessel functions $J(x ; \boldsymbol{\varsigma}, \lambda)$ of doubled rank $2 n$. However, unlike the expression of $J_{(\mu, \delta)}( \pm x)$ by a finite sum of $J\left(2 \pi x^{\frac{1}{n}} ; \boldsymbol{s}, \boldsymbol{\mu}\right)$ as in 3.48$)$, these connection formulae yield an expression of $J_{(\boldsymbol{\mu}, \boldsymbol{m})}\left(x e^{i \phi}\right)$ in terms of an infinite series involving the Bessel functions $J\left(2 \pi x^{\frac{1}{n}} ; \boldsymbol{s}, \lambda\right)$ of rank $2 n$, so a similar reduction for $J_{(\mu, m)}(z)$ does not exist and we need to search for other approaches.

3.5.2. Normalizations of Indices. Usually, it is convenient to normalize the indices in $J(x ; \boldsymbol{s}, \lambda), j_{(\mu, \delta)}(x), j_{(\mu, \boldsymbol{m})}(x), J_{(\mu, \delta)}(x)$ and $J_{(\boldsymbol{\mu}, \boldsymbol{m})}(z)$ so that $\lambda, \boldsymbol{\mu} \in \mathbb{L}^{n-1}$. Furthermore, without loss of generality, the assumptions $\delta_{n}=0$ and $m_{n}=0$ may also be imposed for $J_{(\mu, \delta)}(x)$ and $J_{(\mu, m)}(z)$ respectively. These normalizations are justified by Lemma 3.5 . 3.15, 3.22, Proposition 3.14 (1) and 3.17(1). 


\section{Bessel Functions and Bessel Kernels in Classical Cases}

In Introduction, we have seen the occurrence of the oscillatory exponential function $e(x)$ and classical Bessel functions in the Poisson and Voronoï summation formulae. Using their Mellin-Barnes integral representations, we shall now show that these functions indeed coincide with the Bessel kernels in the real rank-one and rank-two cases defined in $\$ 3$ Furthermore, we shall also find that the complex Bessel kernel of rank one is exactly $e(\operatorname{Tr}(z))=e(z+\bar{z})$.

4.1. Rank-One Bessel Functions. To start with, we have the formula ([GR 3.764])

$$
\Gamma(s) e\left( \pm \frac{s}{4}\right)=\int_{0}^{\infty} e^{ \pm i x} x^{s} d^{\times} x, \quad 0<\Re e s<1 .
$$

Proposition 4.1. Suppose $n=1$. Choose the contour $\mathcal{C}=\mathcal{C}_{0}$ as in Definition 3.2. $\mathcal{C}$ starts from $\rho-i \infty$ and ends at $\rho+i \infty$, with $\rho<-\frac{1}{2}$, and all the nonpositive integers lie on the left side of $\mathrm{C}$. We have

$$
e^{ \pm i x}=\frac{1}{2 \pi i} \int_{\mathcal{C}} \Gamma(s) e\left( \pm \frac{s}{4}\right) x^{-s} d s .
$$

Therefore

$$
J(x ; \pm, 0)=e^{ \pm i x} .
$$

Proof. Let $\Re e z>0$. For $\Re e s>0$, we have the formula

$$
\Gamma(s) z^{-s}=\int_{0}^{\infty} e^{-z x} x^{s} d^{\times} x
$$

where the integral is absolutely convergent. The Mellin inversion formula yields

$$
e^{-z x}=\frac{1}{2 \pi i} \int_{(\sigma)} \Gamma(s) z^{-s} x^{-s} d s, \quad \sigma>0 .
$$

Shifting the contour of integration from $(\sigma)$ to $\mathcal{C}$, one sees that

$$
e^{-z x}=\frac{1}{2 \pi i} \int_{\mathcal{C}} \Gamma(s) z^{-s} x^{-s} d s
$$

Choose $z=e^{\mp\left(\frac{1}{2} \pi-\epsilon\right) i}, \pi>\epsilon>0$. In view of Stirling's asymptotic formula, the convergence of the integral above is uniform in $\epsilon$. Therefore, we obtain 4.2 by letting $\epsilon \rightarrow 0$.

Q.E.D.

Remark 4.2. Observe that the integral in (4.1) is only conditionally convergent, the Mellin inversion formula does not apply in the rigorous sense. Nevertheless, 4.2 should be view as the Mellin inversion of 4.1).

Remark 4.3. It follows from the proof of Proposition 4.1 that the formula

$$
e^{-e(a) x}=\frac{1}{2 \pi i} \int_{\mathcal{C}} \Gamma(s) e(-a s) x^{-s} d s
$$

is valid for any $a \in\left[-\frac{1}{4}, \frac{1}{4}\right]$. 


\subsection{Rank-Two Bessel Functions.}

Proposition 4.4. Let $\lambda \in \mathbb{C}$. Then

$$
\begin{aligned}
& J(x ; \pm, \pm, \lambda,-\lambda)= \pm \pi i e^{ \pm \pi i \lambda} H_{2 \lambda}^{(1,2)}(2 x), \\
& J(x ; \pm, \mp, \lambda,-\lambda)=2 e^{\mp \pi i \lambda} K_{2 \lambda}(2 x) .
\end{aligned}
$$

Here $H_{v}^{(1)}$ and $H_{v}^{(2)}$ are Bessel functions of the third kind, also known as Hankel functions, whereas $K_{v}$ is the modified Bessel function of the second kind, occasionally called the $K$-Bessel function or MacDonald function.

Proof. The following formulae are derived from [GR 6.561 14-16] along with Euler's reflection formula of the Gamma function.

$$
\pi \int_{0}^{\infty} J_{v}(2 \sqrt{x}) x^{s-1} d x=\Gamma\left(s+\frac{v}{2}\right) \Gamma\left(s-\frac{v}{2}\right) \sin \left(\pi\left(s-\frac{v}{2}\right)\right)
$$

for $-\frac{1}{2} \mathfrak{R e} v<\Re$ e $s<\frac{1}{4}$,

$$
-\pi \int_{0}^{\infty} Y_{v}(2 \sqrt{x}) x^{s-1} d x=\Gamma\left(s+\frac{v}{2}\right) \Gamma\left(s-\frac{v}{2}\right) \cos \left(\pi\left(s-\frac{v}{2}\right)\right)
$$

for $\frac{1}{2}|\operatorname{Re} v|<$ Re $s<\frac{1}{4}$, and

$$
2 \int_{0}^{\infty} K_{v}(2 \sqrt{x}) x^{s-1} d x=\Gamma\left(s+\frac{v}{2}\right) \Gamma\left(s-\frac{v}{2}\right)
$$

for $\Re$ e $s>\frac{1}{2}|\Re \mathrm{e} v|$. For $\Re$ e $s$ in the given ranges, these integrals are absolutely convergent. It follows immediately from the Mellin inversion formula that

$$
\begin{aligned}
& J(x ; \pm, \pm, \lambda,-\lambda)= \pm \pi i e^{ \pm \pi i \lambda}\left(J_{2 \lambda}(2 x) \pm i Y_{2 \lambda}(2 x)\right), \quad|\Re \mathrm{e} \lambda|<\frac{1}{4}, \\
& J(x ; \pm, \mp, \lambda,-\lambda)=2 e^{\mp \pi i \lambda} K_{2 \lambda}(2 x) .
\end{aligned}
$$

In view of the analyticity in $\lambda$, the first formula remains valid even if $|\Re e \lambda| \geqslant \frac{1}{4}$ by the theory of analytic continuation. Finally, we conclude the proof by recollecting the connection formula $H_{v}^{(1,2)}(x)=J_{v}(x) \pm i Y_{v}(x)$ in 0.5$)$.

Q.E.D.

4.3. Real Bessel Kernels of Rank One and Two. For $n=1$, using (3.16) and (3.34), Proposition 4.1 implies that

$$
j_{(0,0)}(x)=2 \cos (2 \pi x), \quad j_{(0,1)}(x)=2 i \sin (2 \pi x) .
$$

and

$$
J_{(0,0)}(x)=e(x)
$$

For $n=2$, 3.48) reads

$$
J_{(\mu,-\mu, \delta, 0)}( \pm x)=J(2 \pi \sqrt{x} ;+, \pm, \mu,-\mu)+(-)^{\delta} J(2 \pi \sqrt{x} ;-, \mp, \mu,-\mu),
$$

for $x \in \mathbb{R}_{+}, \mu \in \mathbb{C}$ and $\delta \in \mathbb{Z} / 2 \mathbb{Z}$. In view of Proposition 4.4, for $x \in \mathbb{R}_{+}$, we have

$$
J_{(\mu,-\mu, \delta, 0)}(x)= \begin{cases}-\frac{\pi}{\sin (\pi \mu)}\left(J_{2 \mu}(4 \pi \sqrt{x})-J_{-2 \mu}(4 \pi \sqrt{x})\right), & \text { if } \delta=0, \\ \frac{\pi i}{\cos (\pi \mu)}\left(J_{2 \mu}(4 \pi \sqrt{x})+J_{-2 \mu}(4 \pi \sqrt{x})\right), & \text { if } \delta=1,\end{cases}
$$


where the right hand side is replaced by its limit if $2 \mu \in \delta+2 \mathbb{Z}$, and

$$
J_{(\mu,-\mu, \delta, 0)}(-x)= \begin{cases}4 \cos (\pi \mu) K_{2 \mu}(4 \pi \sqrt{x}), & \text { if } \delta=0, \\ -4 i \sin (\pi \mu) K_{2 \mu}(4 \pi \sqrt{x}), & \text { if } \delta=1 .\end{cases}
$$

Observe that for $m \in \mathbb{N}$

$$
J_{\left(\frac{1}{2} m,-\frac{1}{2} m, \delta(m)+1,0\right)}(x)=2 \pi i^{m+1} J_{m}(4 \pi \sqrt{x}), \quad J_{\left(\frac{1}{2} m,-\frac{1}{2} m, \delta(m)+1,0\right)}(-x)=0 .
$$

Remark 4.5. Let $\mu=i t$ if $F$ is a Maaß form of eigenvalue $\frac{1}{4}+t^{2}$ and weight $k$, and let $\mu=\frac{1}{2}(k-1)$ if $F$ is a holomorphic cusp form of weight $k$. At the real place $F$ may be parametrized by $(\boldsymbol{\mu}, \boldsymbol{\delta})=(\mu,-\mu, k(\bmod 2), 0)$ so that $J_{F}=J_{(\mu, \delta)}$. Hence the formulae of $J_{F}$ in $0.1,0.2,0.3$ are justified.

4.4. Complex Bessel Kernels of Rank One. Let $n=1$. By (3.16) and (3.23), it follows from Proposition 4.4 that

$$
j_{(0, m)}(x)=2 \pi i^{|m|} J_{|m|}(4 \pi x)=2 \pi i^{m} J_{m}(4 \pi x) .
$$

where the second equality follows from the identity $J_{-m}(x)=(-)^{m} J_{m}(x)$. In view of 3.43), the following expansions (Wat $2.22(3,4)]$ )

$$
\begin{aligned}
& \cos (x \cos \phi)=J_{0}(x)+2 \sum_{d=1}^{\infty}(-)^{d} J_{2 d}(x) \cos (2 d \phi), \\
& \sin (x \cos \phi)=2 \sum_{d=0}^{\infty}(-)^{d} J_{2 d+1}(x) \cos ((2 d+1) \phi),
\end{aligned}
$$

imply

$$
J_{(0,0)}\left(x e^{i \phi}\right)=\cos (4 \pi x \cos \phi)+i \sin (4 \pi x \cos \phi)=e(2 x \cos \phi),
$$

or equivalently,

$$
J_{(0,0)}(z)=e(z+\bar{z}) .
$$

We remark that the two expansions [Wat $2.22(3,4)]$ can be incorporated into

$$
e^{i x \cos \phi}=\sum_{m=-\infty}^{\infty} i^{m} J_{m}(x) e^{i m \phi} .
$$

\section{Fourier Type Integral Transforms}

In this section, we shall introduce an alternative perspective of Hankel transforms. We shall first show how to construct Hankel transforms from the Fourier transform and MillerSchmid transforms. From this, we shall express the Hankel transforms $\mathcal{H}_{(\boldsymbol{s}, \lambda)}, \mathcal{H}_{(\mu, \delta)}$ and $\mathcal{H}_{(\mu, m)}$ in terms of certain Fourier type integral transforms, assuming that the components of $\Re e \lambda$ or $\mathfrak{R e} \mu$ are strictly decreasing.

5.1. The Fourier Transform and Rank-One Hankel Transforms. By Proposition 4.1. $J(x ; \pm, 0)=e^{ \pm i x}$ so that the Hankel transform $\mathcal{H}_{( \pm, 0)}$ is in essence a real Fourier transform on $\mathbb{R}_{+}$. Slightly more generally, we want to consider the transform $\mathcal{S}_{(\varsigma, \lambda)} v(x)=$ $x^{\lambda} \mathcal{H}_{(\varsigma, \lambda)} v(x)$.

Lemma 5.1. Let $(\varsigma, \lambda) \in\{+,-\} \times \mathbb{C}$. For $v \in \mathscr{S}\left(\mathbb{R}_{+}\right)$, define $\mathcal{S}_{(\varsigma, \lambda)} v(x)=x^{\lambda} \mathcal{H}_{(\varsigma, \lambda)} v(x)$. Then

$$
\mathcal{M} \mathcal{S}_{(\varsigma, \lambda)} v(s)=G(s, \varsigma) \mathcal{M} v(1-s-\lambda),
$$


and $\mathcal{S}_{(\varsigma, \lambda)}$ sends $\mathscr{S}\left(\mathbb{R}_{+}\right)$into $\mathscr{S}\left(\overline{\mathbb{R}}_{+}\right)$. Furthermore,

$$
\mathcal{S}_{(\varsigma, \lambda)} v(x)=\int_{\mathbb{R}_{+}} y^{-\lambda} v(y) e^{\varsigma i x y} d y .
$$

For either $\mathbb{F}=\mathbb{R}$ or $\mathbb{F}=\mathbb{C}$, we have seen in $\$ 4.3$ and 4.4 that $J_{(0,0)}$ is exactly the inverse Fourier kernel, namely

$$
J_{(0,0)}(x)=e(\Lambda(x)), \quad x \in \mathbb{F},
$$

with $\Lambda(x)$ defined by 1.37). Therefore, in view of Proposition 3.14(3) and 3.17 (3), $\mathcal{H}_{(0,0)}$ is precisely the inverse Fourier transform over the Schwartz space $\mathscr{S}_{\text {sis }}^{(0,0)}\left(\mathbb{F}^{\times}\right)=\mathscr{S}(\mathbb{F})$. The following lemma is a consequence of Theorem 3.12 and 3.15 .

Lemma 5.2. Let $v \in \mathscr{S}(\mathbb{F})$. If $\mathbb{F}=\mathbb{R}$, then the Fourier transform $\hat{v}$ of $v$ can be determined by the following two identities

$$
\mathcal{M}_{\delta} \widehat{v}(s)=(-)^{\delta} G_{\delta}(s) \mathcal{M}_{\delta} v(1-s), \quad \delta \in \mathbb{Z} / 2 \mathbb{Z} .
$$

If $\mathbb{F}=\mathbb{C}$, then the Fourier transform $\hat{v}$ of $v$ can be determined by the following sequence of identities

$$
\mathcal{M}_{-m} \widehat{v}(2 s)=(-)^{m} G_{m}(s) \mathcal{M}_{m} v(2(1-s)), \quad m \in \mathbb{Z} .
$$

It is convenient for our purpose to also introduce the renormalized rank-one Hankel transforms $\mathcal{S}_{(\mu, \epsilon)}$ and $\mathcal{S}_{(\mu, k)}$ as follows.

Lemma 5.3. Let $(\mu, \epsilon) \in \mathbb{C} \times \mathbb{Z} / 2 \mathbb{Z}$ and $(\mu, k) \in \mathbb{C} \times \mathbb{Z}$.

(1). For $v(x) \in \operatorname{sgn}(x)^{\epsilon}|x|^{\mu} \mathscr{S}(\mathbb{R})$, define $\mathcal{S}_{(\mu, \epsilon)} v(x)=|x|^{\mu} \mathcal{H}_{(\mu, \epsilon)} v(x)$. Then

$$
\mathcal{M}_{\delta} \mathcal{S}_{(\mu, \epsilon)} v(s)=G_{\epsilon+\delta}(s) \mathcal{M}_{\delta} v(1-s-\mu), \quad \delta \in \mathbb{Z} / 2 \mathbb{Z},
$$

and $\mathcal{S}_{(\mu, \epsilon)}$ sends $\operatorname{sgn}(x)^{\epsilon}|x|^{\mu} \mathscr{S}(\mathbb{R})$ onto $\operatorname{sgn}(x)^{\epsilon} \mathscr{S}(\mathbb{R})$ bijectively. Furthermore,

$$
\mathcal{S}_{(\mu, \epsilon)} v(x)=\operatorname{sgn}(x)^{\epsilon} \int_{\mathbb{R}^{\times}} \operatorname{sgn}(y)^{\epsilon}|y|^{-\mu} v(y) e(x y) d y=\operatorname{sgn}(x)^{\epsilon} \mathcal{F} \varphi(-x),
$$

with $\varphi(x)=\operatorname{sgn}(x)^{\epsilon}|x|^{-\mu} v(x) \in \mathscr{S}(\mathbb{R})$.

(2). For $v(z) \in[z]^{k}\|z\|^{\mu} \mathscr{S}(\mathbb{C})$, define $\mathcal{S}_{(\mu, k)} v(z)=\|z\|^{\mu} \mathcal{H}_{(\mu, k)} v(z)$. Then

$$
\mathcal{M}_{-m} \mathcal{S}_{(\mu, k)} v(2 s)=G_{k+m}(s) \mathcal{M}_{m} v(2(1-s-\mu)), \quad m \in \mathbb{Z},
$$

and $\mathcal{S}_{(\mu, k)}$ sends $[z]^{k}\|z\|^{\mu} \mathscr{S}(\mathbb{C})$ onto $[z]^{-k} \mathscr{S}(\mathbb{C})$ bijectively. Furthermore,

$$
\mathcal{S}_{(\mu, k)} v(z)=[z]^{-k} \int_{\mathbb{C}^{x}}[u]^{-k}\|u\|^{-\mu} v(u) e(z u+\overline{z u}) d u=[z]^{-k} \mathcal{F} \varphi(-z),
$$

with $\varphi(z)=[z]^{-k}\|z\|^{-\mu} v(z) \in \mathscr{S}(\mathbb{C})$.

Lemma 5.4. Let $(\mu, \epsilon) \in \mathbb{C} \times \mathbb{Z} / 2 \mathbb{Z}$ and $(\mu, k) \in \mathbb{C} \times \mathbb{Z}$.

(1). Let $\delta \in \mathbb{Z} / 2 \mathbb{Z}$. Suppose that $\varphi(x) \in x^{\mu} \mathscr{S}_{\delta+\epsilon}\left(\overline{\mathbb{R}}_{+}\right)$and $v(x)=\operatorname{sgn}(x)^{\delta} \varphi(|x|)$. Then

$$
\begin{aligned}
\mathcal{S}_{(\mu, \epsilon)} v( \pm x)= & ( \pm)^{\delta} \int_{\mathbb{R}_{+}} y^{-\mu} \varphi(y) j_{(0, \delta+\epsilon)}(x y) d y \\
= & \begin{cases}( \pm)^{\epsilon} 2 \int_{\mathbb{R}_{+}} y^{-\mu} \varphi(y) \cos (x y) d y, & \text { if } \delta=\epsilon, \\
( \pm)^{\epsilon+1} 2 i \int_{\mathbb{R}_{+}} y^{-\mu} \varphi(y) \sin (x y) d y, & \text { if } \delta=\epsilon+1 .\end{cases}
\end{aligned}
$$

The transform $\mathcal{S}_{(\mu, \epsilon)}$ is a bijective map from $\operatorname{sgn}(x)^{\epsilon}|x|^{\mu} \mathscr{S}_{\delta}(\mathbb{R})$ onto $\operatorname{sgn}(x)^{\epsilon} \mathscr{S}_{\delta}(\mathbb{R})$. 
(2). Let $m \in \mathbb{Z}$. Suppose that $\varphi(x) \in x^{2 \mu} \mathscr{S}_{-m-k}\left(\overline{\mathbb{R}}_{+}\right)$and $v(z)=[z]^{-m} \varphi(|z|)$. Then

$$
\begin{aligned}
\mathcal{S}_{(\mu, k)} v\left(x e^{i \phi}\right) & =2 e^{i m \phi} \int_{\mathbb{R}_{+}} y^{1-2 \mu} \varphi(y) j_{(0, m+k)}(x y) d y \\
& =4 \pi i^{m+k} e^{i m \phi} \int_{\mathbb{R}_{+}} y^{1-2 \mu} \varphi(y) J_{m+k}(4 \pi x y) d y .
\end{aligned}
$$

The transform $\mathcal{S}_{(\mu, k)}$ is a bijective map from $[z]^{k}\|z\|^{\mu} \mathscr{S}_{m}(\mathbb{C})$ onto $[z]^{-k} \mathscr{S}_{-m}(\mathbb{C})$.

5.2. Miller-Schmid Transforms. Following [MS1, §6], we now define certain transforms over $\mathbb{R}_{+}, \mathbb{R}$ and $\mathbb{C}$. Such transforms over $\mathbb{R}$ have played an important role in the proof of the Voronoï summation formula for $\mathrm{GL}_{n}(\mathbb{Z})$ in their subsequent work [MS3, MS4].

\subsubsection{The Miller-Schmid Transform $\mathcal{T}_{(\varsigma, \lambda)}$.}

Lemma 5.5. Let $(\varsigma, \lambda) \in\{+,-\} \times \mathbb{C}$.

(1). For any $v \in \mathscr{S}_{\text {sis }}\left(\mathbb{R}_{+}\right)$there is a unique function $\Upsilon \in \mathscr{S}_{\text {sis }}\left(\mathbb{R}_{+}\right)$satisfying the following identity,

$$
\mathcal{M} \Upsilon(s)=G(s, \varsigma) \mathcal{M} v(s+\lambda)
$$

We write $\Upsilon=\mathcal{T}_{(\varsigma, \lambda)} v$ and call $\mathcal{T}_{(\varsigma, \lambda)}$ the Miller-Schmid transform over $\mathbb{R}_{+}$of index $(\varsigma, \lambda)$.

(2). Let $v \in \mathbb{C}$. Suppose $v(x) \in x^{-v}(\log x)^{j} \mathscr{S}\left(\overline{\mathbb{R}}_{+}\right)$. If $\mathfrak{R e} v<\Re e \lambda-\frac{1}{2}$, then

$$
\mathcal{T}_{(\varsigma, \lambda)} v(x)=\int_{\mathbb{R}_{+}} y^{-\lambda} v\left(y^{-1}\right) e^{\varsigma i x y} d^{\times} y .
$$

(3). Suppose that $\mathfrak{R e} v<\Re e \lambda$. Then the integral in (5.5) is absolutely convergent and (5.5) remains valid for any $v(x) \in x^{-v}(\log x)^{j} \mathscr{S}\left(\overline{\mathbb{R}}_{+}\right)$.

(4). Suppose that $\mathfrak{R e} \lambda>0$. Define the function space

$$
\mathscr{T}_{\text {sis }}\left(\mathbb{R}_{+}\right)=\sum_{\mathfrak{R e} v \leqslant 0} \sum_{j \in \mathbb{N}} x^{-v}(\log x)^{j} \mathscr{S}\left(\overline{\mathbb{R}}_{+}\right) .
$$

Then the transform $\mathcal{T}_{(\varsigma, \lambda)}$ sends $\mathscr{T}_{\text {sis }}\left(\mathbb{R}_{+}\right)$into itself. Moreover, 5.5) also holds true for any $v \in \mathscr{T}_{\text {sis }}\left(\mathbb{R}_{+}\right)$, wherein the integral absolutely converges.

Proof. Following the ideas in the proof of Proposition 3.4, we may prove (1).

As for (2), we have

$$
\mathcal{T}_{(\varsigma, \lambda)} v(x)=\frac{1}{2 \pi i} \int_{\mathbb{R}_{+}} v(y) y^{\lambda} \int_{\mathcal{C}_{0}} G(s, \varsigma) y^{s} x^{-s} d s d^{\times} y=\int_{\mathbb{R}_{+}} v(y) y^{\lambda} J\left(x y^{-1} ; \varsigma, 0\right) d^{\times} y,
$$

provided that the double integral is absolutely convergent. In order to guarantee the convergence of the integral over $d^{\times} y$, the integral contour $\mathcal{C}_{0}$ is required to lie in the right half-plane $\{s: \mathfrak{R e} s>\mathfrak{R e}(v-\lambda)\}$. In view of Definition 3.2 such a choice of $\mathcal{C}_{0}$ is permissible since $\mathfrak{R e}(v-\lambda)<-\frac{1}{2}$ according to our assumption. Finally, the change of variables from $y$ to $y^{-1}$, along with the formula $J(x ; \varsigma, 0)=e^{\text {six }}$, yields 5.5 .

For the case $\mathfrak{R e} v<\mathfrak{R e} \lambda$ in (3), the absolute convergence of the integral in 5.5) is obvious. The validity of 5.5 follows from the analyticity with respect to $\lambda$.

Under the isomorphism established by $\mathcal{M}$ in Lemma 2.2, $\mathscr{T}_{\text {sis }}\left(\mathbb{R}_{+}\right)$corresponds to the subspace of $\mathscr{M}_{\text {sis }}$ consisting of meromorphic functions $H$ such that the poles of $H$ lie in the left half-plane $\{s: \mathfrak{R e} s \leqslant 0\}$. Hence, the first assertion in (4) is clear, since the map that corresponds to $\mathcal{T}_{(\varsigma, \lambda)}$ is given by $H(s) \mapsto G(s, \varsigma) H(s+\lambda)$ and sends the subspace of $\mathscr{M}_{\text {sis }}$ described above into itself. The second assertion in (4) immediately follows from (3).

Q.E.D. 


\subsubsection{The Miller-Schmid Transform $\mathcal{T}_{(\mu, \epsilon)}$.}

Lemma 5.6. Let $(\mu, \epsilon) \in \mathbb{C} \times \mathbb{Z} / 2 \mathbb{Z}$.

(1). For any $v \in \mathscr{S}_{\text {sis }}\left(\mathbb{R}^{\times}\right)$there is a unique function $\Upsilon \in \mathscr{S}_{\text {sis }}\left(\mathbb{R}^{\times}\right)$satisfying the following two identities,

$$
\mathcal{M}_{\delta} \Upsilon(s)=G_{\epsilon+\delta}(s) \mathcal{M}_{\delta} v(s+\mu), \quad \delta \in \mathbb{Z} / 2 \mathbb{Z}
$$

We write $\Upsilon=\mathcal{T}_{(\mu, \epsilon)} v$ and call $\mathcal{T}_{(\mu, \epsilon)}$ the Miller-Schmid transform over $\mathbb{R}$ of index $(\mu, \epsilon)$.

(2). Let $\lambda \in \mathbb{C}$. Suppose $v(x) \in \operatorname{sgn}(x)^{\delta}|x|^{-\lambda}(\log |x|)^{j} \mathscr{S}(\mathbb{R})$. If $\mathfrak{R e} \lambda<\Re \mathrm{e} \mu-\frac{1}{2}$, then

$$
\mathcal{T}_{(\mu, \epsilon)} v(x)=\operatorname{sgn}(x)^{\epsilon} \int_{\mathbb{R}^{\times}} \operatorname{sgn}(y)^{\epsilon}|y|^{-\mu} v\left(y^{-1}\right) e(x y) d^{\times} y=\operatorname{sgn}(x)^{\epsilon} \mathcal{F} \varphi(-x),
$$

with $\varphi(x)=\operatorname{sgn}(x)^{\epsilon}|x|^{-\mu-1} v\left(x^{-1}\right)$.

(3). Suppose that $\mathfrak{R e} \lambda<\Re e \mu$. Then the integral in 5.7) is absolutely convergent and 5.7) remains valid for any $v(x) \in \operatorname{sgn}(x)^{\delta}|x|^{-\lambda}(\log |x|)^{j} \mathscr{S}(\mathbb{R})$.

(4). Suppose that $\mathfrak{R e} \mu>0$. Define the function space

$$
\mathscr{T}_{\text {sis }}\left(\mathbb{R}^{\times}\right)=\sum_{\delta \in \mathbb{Z} / 2 \mathbb{Z}} \sum_{\Re \mathfrak{R} \lambda \leqslant 0} \sum_{j \in \mathbb{N}} \operatorname{sgn}(x)^{\delta}|x|^{-\lambda}(\log |x|)^{j} \mathscr{S}(\mathbb{R}) .
$$

Then the transform $\mathcal{T}_{(\mu, \epsilon)}$ sends $\mathscr{T}_{\text {sis }}\left(\mathbb{R}^{\times}\right)$into itself. Moreover, 5.7) also holds true for any $v \in \mathscr{T}_{\text {sis }}\left(\mathbb{R}^{\times}\right)$, wherein the integral absolutely converges.

Proof. Following literally the same ideas in the proof of Lemma 5.5, one may show this lemma without any difficulty. Observe that, under the isomorphism established by $\mathcal{M}_{\mathbb{R}}$ in Lemma 2.6. $\mathscr{T}_{\text {sis }}\left(\mathbb{R}^{\times}\right)$corresponds to the subspace of $\mathscr{M}_{\text {sis }}^{\mathbb{R}}$ consisting of pairs of meromorphic functions $\left(H_{0}, H_{1}\right)$ such that the poles of both $H_{0}$ and $H_{1}$ lie in the left halfplane $\{s: \mathfrak{R e} s \leqslant 0\}$ (see Lemma2.7).

Q.E.D.

Similar to Lemma 5.4(1), we have the following lemma.

Lemma 5.7. Let $(\mu, \epsilon) \in \mathbb{C} \times \mathbb{Z} / 2 \mathbb{Z}$ be such that $\Re$ e $\mu>0$. For $\delta \in \mathbb{Z} / 2 \mathbb{Z}$ define $\mathscr{T}_{\text {sis }}^{\delta}\left(\mathbb{R}^{\times}\right)$ to be the space of functions in $\mathscr{T}_{\text {sis }}\left(\mathbb{R}^{\times}\right)$satisfying the condition $(1.17)$. For $v \in \mathscr{T}_{\text {sis }}^{\delta}\left(\mathbb{R}^{\times}\right)$, we write $v(x)=\operatorname{sgn}(x)^{\delta} \varphi(|x|)$. Then

$$
\begin{aligned}
& \mathcal{T}_{(\mu, \epsilon)} v( \pm x)=( \pm)^{\delta} \int_{\mathbb{R}_{+}} y^{-\mu} \varphi\left(y^{-1}\right) j_{(0, \delta+\epsilon)}(x y) d^{\times} y \\
& = \begin{cases}( \pm)^{\delta} 2 \int_{\mathbb{R}_{+}} y^{-\mu} \varphi\left(y^{-1}\right) \cos (x y) d^{\times} y, & \text { if } \delta=\epsilon, \\
( \pm)^{\delta} 2 i \int_{\mathbb{R}_{+}} y^{-\mu} \varphi\left(y^{-1}\right) \sin (x y) d^{\times} y, & \text { if } \delta=\epsilon+1 .\end{cases}
\end{aligned}
$$

The transform $\mathcal{T}_{(\mu, \epsilon)}$ sends $\mathscr{T}_{\text {sis }}^{\delta}\left(\mathbb{R}^{\times}\right)$into itself.

5.2.3. The Miller-Schmid Transform $\mathcal{T}_{(\mu, k)}$. In parallel to Lemma 5.6 the following lemma defines the Miller-Schmid transform $\mathcal{T}(\mu, k)$ over $\mathbb{C}$ and gives its connection to the Fourier transform over $\mathbb{C}$.

Lemma 5.8. Let $(\mu, k) \in \mathbb{C} \times \mathbb{Z}$.

(1). For any $v \in \mathscr{S}_{\text {sis }}\left(\mathbb{C}^{\times}\right)$there is a unique function $\Upsilon \in \mathscr{S}_{\text {sis }}\left(\mathbb{C}^{\times}\right)$satisfying the following sequence of identities,

$$
\mathcal{M}_{-m} \Upsilon(2 s)=G_{m+k}(s) \mathcal{M}_{-m} v(2(s+\mu)), \quad m \in \mathbb{Z} .
$$

We write $\Upsilon=\mathcal{T}_{(\mu, k)} v$ and call $\mathcal{T}_{(\mu, k)}$ the Miller-Schmid transform over $\mathbb{C}$ of index $(\mu, k)$. 
(2). Let $\lambda \in \mathbb{C}$. If $\Re e x<2 \Re e \mu$, then for any $v(z) \in[z]^{m}|z|^{-\lambda}(\log |z|)^{j} \mathscr{S}(\mathbb{C})$ we have

$$
\mathcal{T}_{(\mu, k)} v(z)=[z]^{k} \int_{\mathbb{C}^{\times}}[u]^{k}\|u\|^{-\mu} v\left(u^{-1}\right) e(z u+\overline{z u}) d^{\times} u=[z]^{k} \mathcal{F} \varphi(-z),
$$

with $\varphi(z)=[z]^{k}\|z\|^{-\mu-1} v\left(z^{-1}\right)$.

(3). When Re $\lambda<2 \Re e \mu$, the integral in (5.9) is absolutely convergent for any $v(z) \in$ $[z]^{m}|z|^{-\lambda}(\log |z|)^{j} \mathscr{S}(\mathbb{C})$.

(4). Suppose that $\mathfrak{R e} \mu>0$. Define the function space

$$
\mathscr{T}_{\text {sis }}\left(\mathbb{C}^{\times}\right)=\sum_{m \in \mathbb{Z}} \sum_{\mathfrak{R} \mathrm{e} \lambda \leqslant 0} \sum_{j \in \mathbb{N}}[z]^{m}|z|^{-\lambda}(\log |z|)^{j} \mathscr{S}(\mathbb{C}) .
$$

Then the transform $\mathcal{T}_{(\mu, k)}$ sends $\mathscr{T}_{\text {sis }}\left(\mathbb{C}^{\times}\right)$into itself. Moreover, (5.9) also holds true for any $v \in \mathscr{T}_{\text {sis }}\left(\mathbb{C}^{\times}\right)$, wherein the integral absolutely converges.

Proof. Again, the proof is similar as above. We only remark that, via the isomorphism $\mathcal{M}_{\mathbb{C}}$ in Lemma $2.8, \mathscr{T}_{\text {sis }}\left(\mathbb{C}^{\times}\right)$corresponds to the subspace of $\mathscr{M}_{\text {sis }}^{\mathbb{C}}$ consisting of sequences $\left\{H_{m}\right\}$ such that the poles of each $H_{m}$ lie in the left half-plane $\{s: \Re e s \leqslant \min \{M-|m|, 0\}\}$ for some $M \in \mathbb{N}$ (see Lemma 2.9.

Q.E.D.

Lemma 5.9. Let $(\mu, k) \in \mathbb{C} \times \mathbb{Z}$ be such that $\mathfrak{R e} \mu>0$. For $m \in \mathbb{Z}$ define $\mathscr{T}_{\text {sis }}^{m}\left(\mathbb{C}^{\times}\right)$to be the space of functions in $\mathscr{T}_{\text {sis }}\left(\mathbb{C}^{\times}\right)$satisfying the condition 1.21 . For $v \in \mathscr{T}_{\text {sis }}^{m}\left(\mathbb{C}^{\times}\right)$, we write $v(z)=[z]^{m} \varphi(|z|)$. Then

$$
\begin{aligned}
\mathcal{T}_{(\mu, k)} v\left(x e^{i \phi}\right) & =2 e^{i m \phi} \int_{\mathbb{R}_{+}} y^{-2 \mu} \varphi\left(y^{-1}\right) j_{(0, m+k)}(x y) d^{\times} y \\
& =4 \pi i^{m+k} e^{i m \phi} \int_{\mathbb{R}_{+}} y^{-2 \mu} \varphi\left(y^{-1}\right) J_{m+k}(4 \pi x y) d^{\times} y .
\end{aligned}
$$

The transform $\mathcal{T}_{(\mu, k)}$ sends $\mathscr{T}_{\text {sis }}^{m}\left(\mathbb{C}^{\times}\right)$into itself.

5.3. Fourier Type Integral Transforms. Following [MS3, MS4], we shall now derive the Fourier type integral transform expressions for $\mathcal{H}_{(\boldsymbol{s}, \lambda)}, \mathcal{H}_{(\mu, \delta)}$ and $\mathcal{H}_{(\mu, \boldsymbol{m})}$ from the Fourier transform (more precisely, the renormalized rank-one Hankel transforms) and the Miller-Schmid transforms.

5.3.1. The Fourier Type Transform Expression for $\mathcal{H}_{(\varsigma, \lambda)}$. Let $(\boldsymbol{s}, \lambda) \in\{+,-\}^{n} \times \mathbb{C}^{n}$. For $v \in \mathscr{S}\left(\mathbb{R}_{+}\right)$, we put

$$
\Upsilon(x)=x^{-\lambda_{1}} \mathcal{T}_{\left(\varsigma_{1}, \lambda_{1}-\lambda_{2}\right)}{ }^{\circ} \ldots \circ \mathcal{T}_{\left(\varsigma_{n-1}, \lambda_{n-1}-\lambda_{n}\right)} \circ \mathcal{S}_{\left(\varsigma_{n}, \lambda_{n}\right)} v(x) .
$$

According to Lemma 5.1 and Lemma $5.5(1), \mathcal{S}_{\left(\varsigma_{n}, \lambda_{n}\right)} v(x)$ lies in the space $\mathscr{S}\left(\overline{\mathbb{R}}_{+}\right)(\subset$ $\mathscr{S}_{\text {sis }}\left(\mathbb{R}_{+}\right)$), whereas each Miller-Schmid transform sends $\mathscr{S}_{\text {sis }}\left(\mathbb{R}_{+}\right)$into itself. Thus one can apply the Mellin transform $\mathcal{M}$ to both sides of (5.10). Using (5.1) and (5.4), some calculations show that the application of $\mathcal{M}$ converts (5.10) exactly into the identity (3.3) which defines $\mathcal{H}_{(\boldsymbol{\varsigma}, \lambda)}$. Therefore, $\Upsilon=\mathcal{H}_{(\boldsymbol{\varsigma}, \lambda)} v$.

Theorem 5.10. Let $(\boldsymbol{s}, \lambda) \in\{+,-\}^{n} \times \mathbb{C}^{n}$ be such that $\mathfrak{R e} \lambda_{1}>\ldots>$ Re $\lambda_{n-1}>\operatorname{Re} \lambda_{n}$. Suppose $v \in \mathscr{S}\left(\mathbb{R}_{+}\right)$. Then

$$
\mathcal{H}_{(\boldsymbol{s}, \lambda)} v(x)=\frac{1}{x} \int_{\mathbb{R}_{+}^{n}} v\left(\frac{x_{1} \ldots x_{n}}{x}\right)\left(\prod_{l=1}^{n} x_{l}^{-\lambda_{l}} e^{\varsigma_{l} i x_{l}}\right) d x_{n} \ldots d x_{1},
$$

where the integral converges when performed as iterated integral in the indicated order $d x_{n} d x_{n-1} \ldots d x_{1}$, starting from $d x_{n}$, then $d x_{n-1}, \ldots$, and finally $d x_{1}$. 
Proof. We first observe that $\mathcal{S}_{\left(\varsigma_{n}, \lambda_{n}\right)} v(x) \in \mathscr{S}\left(\overline{\mathbb{R}}_{+}\right) \subset \mathscr{T}_{\text {sis }}\left(\mathbb{R}_{+}\right)$. For each $l=1, \ldots, n-$ 1, since $\mathfrak{R e}\left(\lambda_{l}-\lambda_{l+1}\right)>0$, Lemma 5.5 (4) implies that the transform $\mathcal{T}_{\left(\zeta_{l}, \lambda_{l}-\lambda_{l+1}\right)}$ sends the space $\mathscr{T}_{\text {sis }}\left(\mathbb{R}_{+}\right)$into itself. According to Lemma 5.1 and Lemma 5.5 (3), $\mathcal{S}_{\left(S_{n}, \lambda_{n}\right)}$ and all the $\mathcal{T}_{\left(\varsigma_{l}, \lambda_{l}-\lambda_{l+1}\right)}$ in 5.10 may be expressed as integral transforms, which are absolutely convergent. From these, the right hand side of 5.10) turns into the integral,

$$
\int_{\mathbb{R}_{+}^{n}} x^{-\lambda_{1}} e^{\varsigma_{1} i x y_{1}}\left(\prod_{l=1}^{n-1} y_{l}^{\lambda_{l+1}-\lambda_{l}-1} e^{S i y_{l}^{-1} y_{l+1}}\right) y_{n}^{-\lambda_{n}} v\left(y_{n}\right) d y_{n} \ldots d y_{1},
$$

which converges as iterated integral. Our proof is completed upon making the change of variables $x_{1}=x y_{1}, x_{l+1}=y_{l}^{-1} y_{l+1}, l=1, \ldots, n-1$.

Q.E.D.

5.3.2. The Fourier Type Transform Expression for $\mathcal{H}_{(\mu, \delta)}$. Let $(\boldsymbol{\mu}, \boldsymbol{\delta}) \in \mathbb{C}^{n} \times(\mathbb{Z} / 2 \mathbb{Z})^{n}$. For $v(x) \in \operatorname{sgn}(x)^{\delta_{n}}|x|^{\mu_{n}} \mathscr{S}(\mathbb{R})$, by Lemma 5.3 (1) and Lemma 5.6 (1), in particular 5.2 and (5.6), we may prove that

$$
\mathcal{H}_{(\mu, \delta)} v(x)=|x|^{-\mu_{1}} \mathcal{T}_{\left(\mu_{1}-\mu_{2}, \delta_{1}\right)} \circ \ldots \circ \mathcal{T}_{\left(\mu_{n-1}-\mu_{n}, \delta_{n-1}\right)}{ }^{\circ} \mathcal{S}_{\left(\mu_{n}, \delta_{n}\right)} v(x) .
$$

Theorem 5.11. [MS4, (1.3)]. Let $(\boldsymbol{\mu}, \boldsymbol{\delta}) \in \mathbb{C}^{n} \times(\mathbb{Z} / 2 \mathbb{Z})^{n}$ be such that $\Re$ e $\mu_{1}>\ldots>$ $\mathfrak{R e} \mu_{n-1}>\mathfrak{R e} \mu_{n}$. Suppose $v(x) \in \operatorname{sgn}(x)^{\delta_{n}}|x|^{\mu_{n}} \mathscr{S}(\mathbb{R})$. Then

$$
\mathcal{H}_{(\mu, \delta)} v(x)=\frac{1}{|x|} \int_{\mathbb{R}^{\times n}} v\left(\frac{x_{1} \ldots x_{n}}{x}\right)\left(\prod_{l=1}^{n} \operatorname{sgn}\left(x_{l}\right)^{\delta_{l}}\left|x_{l}\right|^{-\mu_{l}} e\left(x_{l}\right)\right) d x_{n} \ldots d x_{1},
$$

where the integral converges when performed as iterated integral in the indicated order $d x_{n} d x_{n-1} \ldots d x_{1}$, starting from $d x_{n}$, then $d x_{n-1}, \ldots$, and finally $d x_{1}$.

Proof. One applies the same arguments in the proof of Theorem 5.10 using Lemma 5.3 (1) and Lemma 5.6 $(3,4)$.

Q.E.D.

We have the following corollary to Theorem 5.11. which can also be seen from Lemma 5.4 (1) and Lemma 5.7

Corollary 5.12. Let $(\boldsymbol{\mu}, \boldsymbol{\delta}) \in \mathbb{C}^{n} \times(\mathbb{Z} / 2 \mathbb{Z})^{n}$ and $\delta \in \mathbb{Z} / 2 \mathbb{Z}$. Assume that $\Re \mathrm{Re} \mu_{1}>\ldots>$ $\Re e \mu_{n-1}>\Re e \mu_{n}$. Let $\varphi(x) \in x^{\mu_{n}} \mathscr{S}_{\delta+\delta_{n}}\left(\overline{\mathbb{R}}_{+}\right)$and $v(x)=\operatorname{sgn}(x)^{\delta} \varphi(|x|)$. Then

$$
\mathcal{H}_{(\mu, \boldsymbol{m})} v( \pm x)=\frac{( \pm)^{\delta}}{x} \int_{\mathbb{R}_{+}^{n}} \varphi\left(\frac{x_{1} \ldots x_{n}}{x}\right)\left(\prod_{l=1}^{n} x_{l}^{-\mu_{l}} j_{\left(0, \delta_{l}+\delta\right)}\left(x_{l}\right)\right) d x_{n} \ldots d x_{1},
$$

with $x \in \mathbb{R}_{+}$. Here the iterated integration is performed in the indicated order.

5.3.3. The Fourier Type Transform Expression for $\mathcal{H}_{(\mu, \boldsymbol{m})}$. Let $(\boldsymbol{\mu}, \boldsymbol{m}) \in \mathbb{C}^{n} \times \mathbb{Z}^{n}$. For $v(z) \in[z]^{m_{n}}\|z\|^{\mu_{n}} \mathscr{S}(\mathbb{C})$, using Lemma 5.3 (2) and Lemma 5.8 (1), especially (5.3) and (5.8), one may show that

$$
\mathcal{H}_{(\boldsymbol{\mu}, \boldsymbol{m})} v(z)=\|z\|^{-\mu_{1}} \mathcal{T}_{\left(\mu_{1}-\mu_{2}, m_{1}\right)}{ }^{\circ} \ldots \circ \mathcal{T}_{\left(\mu_{n-1}-\mu_{n}, m_{n-1}\right)} \circ \mathcal{S}_{\left(\mu_{n}, m_{n}\right)} v(z) .
$$

Theorem 5.13. Let $(\boldsymbol{\mu}, \boldsymbol{m}) \in \mathbb{C}^{n} \times \mathbb{Z}^{n}$ be such that $\mathfrak{R e} \mu_{1}>\ldots>\mathfrak{R e} \mu_{n-1}>\mathfrak{R e} \mu_{n}$. Suppose $v(z) \in[z]^{m_{n}}\|z\|^{\mu_{n}} \mathscr{S}(\mathbb{C})$. Then

$$
\mathcal{H}_{(\boldsymbol{\mu}, \boldsymbol{m})} v(z)=\frac{1}{\|z\|} \int_{\mathbb{C}^{\times n}} v\left(\frac{z_{1} \ldots z_{n}}{z}\right)\left(\prod_{l=1}^{n}\left[z_{l}\right]^{-m_{l}}\left\|z_{l}\right\|^{-\mu_{l}} e\left(z_{l}+\overline{z_{l}}\right)\right) d z_{n} \ldots d z_{1},
$$

where the integral converges when performed as iterated integral in the indicated order.

Proof. One applies the same arguments in the proof of Theorem 5.10 using Lemma 5.3 (2) and Lemma 5.8 $(3,4)$.

Q.E.D. 
Theorem 5.13, or Lemma 5.4 (2) and Lemma 5.9, yields the following corollary.

Corollary 5.14. Let $(\boldsymbol{\mu}, \boldsymbol{m}) \in \mathbb{C}^{n} \times \mathbb{Z}^{n}$ and $m \in \mathbb{Z}$. Assume that $\mathfrak{R e} \mu_{1}>\ldots>$ $\mathfrak{R e} \mu_{n-1}>\mathfrak{R e} \mu_{n}$. Let $\varphi(x) \in x^{2 \mu_{n}} \mathscr{S}_{-m-m_{n}}\left(\overline{\mathbb{R}}_{+}\right)$and $v(z)=[z]^{-m} \varphi(|z|)$. Then

$$
\mathcal{H}_{(\mu, \boldsymbol{m})} v\left(x e^{i \phi}\right)=2^{n} \frac{e^{i m \phi}}{x^{2}} \int_{\mathbb{R}_{+}^{n}} \varphi\left(\frac{x_{1} \ldots x_{n}}{x}\right)\left(\prod_{l=1}^{n} x_{l}^{-2 \mu_{l}+1} j_{\left(0, m_{l}+m\right)}\left(x_{l}\right)\right) d x_{n} \ldots d x_{1},
$$

with $x \in \mathbb{R}_{+}$and $\phi \in \mathbb{R} / 2 \pi \mathbb{Z}$. Here the iterated integration is performed in the indicated order.

\section{Integral Representations of Bessel Kernels}

First, we shall derive a formal integral representation of the Bessel function $J(x ; \boldsymbol{c}, \lambda)$ from Theorem 5.10 in symbolic manner, ignoring the assumption $\mathfrak{R e} \lambda_{1}>\ldots>\mathfrak{R e} \lambda_{n}$. This formal integral will be the subject that we study in $\$ 7$ - 9 of Chapter 2

Similarly, Theorem 5.11 and 5.13 also yield formal integral representations of $J_{(\mu, \delta)}(x)$ and $J_{(\mu, m)}(z)$ respectively. It turns out that one can naturally transform the formal integral of $J_{(\mu, m)}(z)$ into an integral that is absolutely convergent, given that the index $\boldsymbol{\mu}$ satisfies certain conditions. The main reason for the absolute convergence is that $j_{(0, m)}(x)=$ $2 \pi i^{m} J_{m}(4 \pi x)$ (see (4.4)) decays proportionally to $1 / \sqrt{x}$ at infinity (in comparison, $j_{(0, \delta)}(x)$ is equal to either $2 \cos (2 \pi x)$ or $2 i \sin (2 \pi x))$.

Assumptions and Notations. Let $n \geqslant 2$. Assume that $\lambda, \boldsymbol{\mu} \in \mathbb{L}^{n-1}$.

Notation 6.1. Let $d=n-1$. Let the pairs of tuples, $\lambda$ or $\boldsymbol{\mu} \in \mathbb{L}^{d}$ and $\boldsymbol{v} \in \mathbb{C}^{d}$, $\boldsymbol{\delta} \in(\mathbb{Z} / 2 \mathbb{Z})^{d+1}$ and $\boldsymbol{\epsilon} \in(\mathbb{Z} / 2 \mathbb{Z})^{d}, \boldsymbol{m} \in \mathbb{Z}^{d+1}$ and $\boldsymbol{k} \in \mathbb{Z}^{d}$, be subjected to the following relations

$$
v_{l}=\lambda_{l}-\lambda_{d+1}, \quad v_{l}=\mu_{l}-\mu_{d+1}, \quad \epsilon_{l}=\delta_{l}+\delta_{d+1}, \quad k_{l}=m_{l}-m_{d+1},
$$

for $l=1, \ldots, d$.

Rather than Hankel transforms, we shall be interested in their Bessel kernels. Therefore, it is convenient to further assume that the weight functions are Schwartz, namely, $\varphi \in \mathscr{S}\left(\mathbb{R}_{+}\right)$and $v \in \mathscr{S}\left(\mathbb{F}^{\times}\right)$. According to 3.5, 3.20, 3.27), Proposition 3.14 (3) and 3.17 (3), for such Schwartz functions $\varphi$ and $v$,

$$
\begin{aligned}
& \mathcal{H}_{(\boldsymbol{s}, \lambda)} \varphi(x)=\int_{\mathbb{R}_{+}} \varphi(y) J\left((x y)^{\frac{1}{n}} ; \boldsymbol{\varsigma}, \lambda\right) d y, \\
& \digamma_{(\mu, \delta)} \varphi(x)=\int_{\mathbb{R}_{+}} \varphi(y) j_{(\mu, \delta)}(x y) d y, \quad h_{(\mu, \boldsymbol{m})} \varphi(x)=2 \int_{\mathbb{R}_{+}} \varphi(y) j_{(\mu, \boldsymbol{m})}(x y) y d y, \\
& \mathcal{H}_{(\boldsymbol{\mu}, \boldsymbol{\delta})} v(x)=\int_{\mathbb{R}^{x}} v(y) J_{(\boldsymbol{\mu}, \boldsymbol{\delta})}(x y) d y, \quad \mathcal{H}_{(\boldsymbol{\mu}, \boldsymbol{m})} v(z)=\int_{\mathbb{C}^{x}} v(u) J_{(\boldsymbol{\mu}, \boldsymbol{m})}(z u) d u,
\end{aligned}
$$

with indices $(\boldsymbol{\varsigma}, \lambda) \in\{+,-\}^{n} \times \mathbb{C}^{n},(\boldsymbol{\mu}, \boldsymbol{\delta}) \in \mathbb{C}^{n} \times(\mathbb{Z} / 2 \mathbb{Z})^{n}$ and $(\boldsymbol{\mu}, \boldsymbol{m}) \in \mathbb{C}^{n} \times \mathbb{Z}^{n}$ being arbitrary.

6.1. The Formal Integral $J_{v}(x ; \boldsymbol{\varsigma})$. To motivate the definition of $J_{v}(x ; \boldsymbol{\varsigma})$, we shall do certain operations on the Fourier type integral (5.11) in Theorem 5.10 In the meanwhile, we shall ignore the assumption $\mathfrak{R e} \lambda_{1}>\ldots>\mathfrak{R e} \lambda_{n}$. 
Upon making the change of variables, $x_{n}=\left(x_{1} \ldots x_{n-1}\right)^{-1} x y, x_{l}=y_{l}^{-1}, l=1, \ldots, n-1$, one converts 5.11 into

$$
\mathcal{H}_{(\boldsymbol{s}, \lambda)} v(x)=\int_{\mathbb{R}_{+}^{n}} v(y)(x y)^{-\lambda_{n}}\left(\prod_{l=1}^{n-1} y_{l}^{\lambda_{l}-\lambda_{n}-1}\right) e^{i\left(\varsigma_{n} x y y_{1} \ldots y_{n-1}+\sum_{l=1}^{n-1} s l y_{l}^{-1}\right)} d y d y_{n-1} \ldots d y_{1} .
$$

In symbolic notation, moving the integral over $d y$ to the outermost place and comparing the resulting integral with the right hand side of 6.1 , the Bessel function $J(x ; \boldsymbol{\varsigma}, \lambda)$ is then represented by the following formal integral over $d y_{n-1} \ldots d y_{1}$,

$$
\int_{\mathbb{R}_{+}^{n-1}} x^{-n \lambda_{n}}\left(\prod_{l=1}^{n-1} y_{l}^{\lambda_{l}-\lambda_{n}-1}\right) e^{i\left(\varsigma_{n} x^{n} y_{1} \ldots y_{n-1}+\sum_{l=1}^{n-1} s y_{l}^{-1}\right)} d y_{n-1} \ldots d y_{1}
$$

Another change of variables $y_{l}=t_{l} x^{-1}$, along with the assumption $\sum_{l=1}^{n} \lambda_{l}=0$, turns this integral into

$$
\int_{\mathbb{R}_{+}^{n-1}}\left(\prod_{l=1}^{n-1} t_{l}^{\lambda_{l}-\lambda_{n}-1}\right) e^{i x\left(\varsigma_{n} t_{1} \ldots t_{n-1}+\sum_{l=1}^{n-1} \varsigma_{l} t_{l}^{-1}\right)} d t_{n-1} \ldots d t_{1} .
$$

For $\boldsymbol{v} \in \mathbb{C}^{d}$ and $\boldsymbol{\varsigma} \in\{+,-\}^{d+1}$, we define the formal integral

$$
J_{v}(x ; \boldsymbol{S})=\int_{\mathbb{R}_{+}^{d}}\left(\prod_{l=1}^{d} t_{l}^{\nu_{l}-1}\right) e^{i x\left(s_{d+1} t_{1} \ldots t_{d}+\sum_{l=1}^{d} S t_{l}^{-1}\right)} d t_{d} \ldots d t_{1}, \quad x \in \mathbb{R}_{+} .
$$

In view of Notation 6.1, we have $J(x ; \boldsymbol{\varsigma}, \lambda)=J_{v}(x ; \boldsymbol{\varsigma})$ in symbolic notation.

Remark 6.2. Before realizing its connection with the Fourier type transform 5.11) in Theorem 5.10, the formal integral representation $J_{v}(x ; \boldsymbol{\varsigma})$ of $J(x ; \boldsymbol{\mathcal { S }}, \lambda)$ was derived by the author from (3.3) in Proposition 3.4 based on a symbolic application of the productconvolution principle of the Mellin transform together with the formula

$$
\Gamma(s) e\left( \pm \frac{s}{4}\right)=\int_{0}^{\infty} e^{ \pm i x} x^{s} d^{\times} x, \quad 0<\mathfrak{R e} s<1 .
$$

Though not specified, this principle is implicitly suggested in Miller and Schmid's work, especially, [MS1, Theorem 4.12, Lemma 6.19] and [MS3, (5.22, 5.26)].

6.2. The Formal Integral $J_{v, \epsilon}(x, \pm)$. We may proceed in the same way as in $\$ 6.1$ starting from the Fourier type integral (5.13) in Theorem 5.11. For $\boldsymbol{v} \in \mathbb{C}^{d}$ and $\epsilon \in(\mathbb{Z} / 2 \mathbb{Z})^{d}$, we define the formal integral

$$
J_{\nu, \epsilon}(x, \pm)=\int_{\mathbb{R}^{\times d}}\left(\prod_{l=1}^{d} \operatorname{sgn}\left(y_{l}\right)^{\epsilon_{l}}\left|y_{l}\right|^{\nu_{l}-1}\right) e^{i x\left( \pm y_{1} \ldots y_{d}+\sum_{l=1}^{d} y_{l}^{-1}\right)} d y_{d} \ldots d y_{1}, \quad x \in \mathbb{R}_{+} .
$$

Then follows the symbolic identity $J_{(\mu, \delta)}( \pm x)=( \pm)^{\delta_{d+1}} J_{\boldsymbol{\nu}, \boldsymbol{\epsilon}}\left(2 \pi x^{\frac{1}{d+1}}, \pm\right)$.

By splitting $\mathbb{R}^{\times}=\mathbb{R}_{+} \cup\left(-\mathbb{R}_{+}\right)$in the domain of integral and letting $y_{l}=s_{l} t_{l}$, some formal calculations yield the following formula

$$
J_{v, \epsilon}(x ; \pm)=\sum_{\boldsymbol{s} \in\{+,-\}^{d}} \boldsymbol{\varsigma}^{\epsilon} J_{v}(x ; \pm|\boldsymbol{s}|),
$$

which corresponds to the formula 3.48 for $J_{(\mu, \delta)}(x)$ and $J\left(2 \pi x^{\frac{1}{d+1}} ; \boldsymbol{\varsigma}, \boldsymbol{\mu}\right)$. 
6.3. The Formal Integral $j_{v, \delta}(x)$. For $\boldsymbol{v} \in \mathbb{C}^{d}$ and $\delta \in(\mathbb{Z} / 2 \mathbb{Z})^{d+1}$, we define the formal integral

$$
j_{v, \delta}(x)=\int_{\mathbb{R}_{+}^{d}} j_{\left(0, \delta_{d+1}\right)}\left(x y_{1} \ldots y_{d}\right) \prod_{l=1}^{d} y_{l}^{v_{l}-1} j_{\left(0, \delta_{l}\right)}\left(x y_{l}^{-1}\right) d y_{d} \ldots d y_{1}, \quad x \in \mathbb{R}_{+} .
$$

We may derive the symbolic identity $j_{(\mu, \delta)}(x)=j_{\nu, \delta}\left(x^{\frac{1}{d+1}}\right)$ from Corollary 3.13 and 5.12

6.4. The Integral $J_{v, k}(x, u)$. First of all, proceeding in the same way as in $\$ 6.1$, from the Fourier type integral 5.16 in Theorem 5.13, we can deduce the symbolic equality $J_{(\mu, \boldsymbol{m})}\left(x e^{i \phi}\right)=e^{-i m_{d+1} \phi} J_{v, k}\left(2 \pi x^{\frac{1}{d+1}}, e^{i \phi}\right)$, with the definition of the formal integral,

$$
\begin{array}{r}
J_{v, k}(x, u)=\int_{\mathbb{C}^{\times d}}\left(\prod_{l=1}^{d}\left[u_{l}\right]^{k_{l}}\left\|u_{l}\right\|^{v_{l}-1}\right) e^{i x \Lambda\left(u u_{1} \ldots u_{d}+\sum_{l=1}^{d} u_{l}^{-1}\right)} d u_{d} \ldots d u_{1}, \\
x \in \mathbb{R}_{+}, u \in \mathbb{C},|u|=1 .
\end{array}
$$

Here, we recall that $\Lambda(z)=z+\bar{z}$.

In the polar coordinates, we write $u_{l}=y_{l} e^{i \theta_{l}}$ and $u=e^{i \phi}$. Moving the integral over the torus $(\mathbb{R} / 2 \pi \mathbb{Z})^{d}$ inside, in symbolic manner, the integral above turns into

$2^{d} \int_{\mathbb{R}_{+}^{d}} \int_{(\mathbb{R} / 2 \pi \mathbb{Z})^{d}}\left(\prod_{l=1}^{d} y_{l}^{2 v_{l}-1}\right) e^{i \sum_{l=1}^{d} k_{l} \theta_{l}+2 i x\left(y_{1} \ldots y_{d} \cos \left(\sum_{l=1}^{d} \theta_{l}+\phi\right)+\sum_{l=1}^{d} y_{l}^{-1} \cos \theta_{l}\right)} d \theta_{d} \ldots d \theta_{1} d y_{d} \ldots d y_{1}$.

Let us introduce the following definitions

$$
\begin{gathered}
\Theta_{\boldsymbol{k}}(\boldsymbol{\theta}, \boldsymbol{y} ; x, \phi)=2 x y_{1} \ldots y_{d} \cos \left(\sum_{l=1}^{d} \theta_{l}+\phi\right)+\sum_{l=1}^{d}\left(k_{l} \theta_{l}+2 x y_{l}^{-1} \cos \theta_{l}\right), \\
J_{\boldsymbol{k}}(\boldsymbol{y} ; x, \phi)=\int_{(\mathbb{R} / 2 \pi \mathbb{Z})^{d}} e^{i \Theta_{k}(\boldsymbol{\theta}, \boldsymbol{y} ; x, \phi)} d \boldsymbol{\theta} \\
p_{2 v}(\boldsymbol{y})=\prod_{l=1}^{d} y_{l}^{2 v_{l}-1}
\end{gathered}
$$

with $\boldsymbol{y}=\left(y_{1}, \ldots, y_{d}\right), \boldsymbol{\theta}=\left(\theta_{1}, \ldots, \theta_{d}\right)$. Then 6.8 can be symbolically rewritten as

$$
J_{\boldsymbol{v}, \boldsymbol{k}}\left(x, e^{i \phi}\right)=2^{d} \int_{\mathbb{R}_{+}^{d}} p_{2 v}(\boldsymbol{y}) J_{\boldsymbol{k}}(\boldsymbol{y} ; x, \phi) d \boldsymbol{y}, \quad x \in \mathbb{R}_{+}, \phi \in \mathbb{R} / 2 \pi \mathbb{Z} .
$$

Theorem 6.3. Let $(\boldsymbol{\mu}, \boldsymbol{m}) \in \mathbb{L}^{d} \times \mathbb{Z}^{d+1}$ and $(\boldsymbol{v}, \boldsymbol{k}) \in \mathbb{C}^{d} \times \mathbb{Z}^{d}$ satisfy the relations given in Notation 6.1 Suppose that $v$ lies in the union

$$
\bigcup_{a \in\left[-\frac{1}{2}, 0\right]}\left\{v \in \mathbb{C}^{d}:-\frac{1}{2}<2 \Re \mathrm{e} v_{l}+a<0 \text { for all } l=1, \ldots, d\right\} .
$$

(1). The integral in 6.12 converges absolutely. Subsequently, we shall therefore use (6.12) as the definition of $J_{v, k}\left(x, e^{i \phi}\right)$.

(2). We have the (genuine) identity

$$
J_{(\mu, \boldsymbol{m})}\left(x e^{i \phi}\right)=e^{-i m_{d+1} \phi} J_{\nu, k}\left(2 \pi x^{\frac{1}{d+1}}, e^{i \phi}\right) .
$$


6.5. The Integral $j_{v, m}(x)$. Let us consider the integral $j_{v, m}(x)$ defined by

$$
j_{v, \boldsymbol{m}}(x)=2^{d} \int_{\mathbb{R}_{+}^{d}} j_{\left(0, m_{d+1}\right)}\left(x y_{1} \ldots y_{d}\right) \prod_{l=1}^{d} y_{l}^{2 v_{l}-1} j_{\left(0, m_{l}\right)}\left(x y_{l}^{-1}\right) d y_{d} \ldots d y_{1},
$$

with $\boldsymbol{v} \in \mathbb{C}^{d}$ and $\boldsymbol{m} \in \mathbb{Z}^{d+1}$.

6.5.1. Absolute convergence of $j_{v, m}(x)$. In contrast to the real case, where the integral $j_{v, \delta}(x)$ never absolutely converges, $j_{v, m}(x)$ is actually absolutely convergent, if each component of $v$ lies in certain vertical strips of width at least $\frac{1}{4}$.

Definition 6.4. For $\boldsymbol{a}, \boldsymbol{b} \in \mathbb{R}^{d}$ such that $a_{l}<b_{l}$ for all $l=1, \ldots, d$, we define the open hyper-strip $\mathbb{S}^{d}(\boldsymbol{a}, \boldsymbol{b})=\left\{\boldsymbol{v} \in \mathbb{C}^{d}: \mathfrak{R e} v_{l} \in\left(a_{l}, b_{l}\right)\right\}$. We write $\mathbb{S}^{d}(a, b)=\mathbb{S}^{d}\left(a \boldsymbol{e}^{d}, b \boldsymbol{e}^{d}\right)$ for simplicity.

Proposition 6.5. Let $(\boldsymbol{v}, \boldsymbol{m}) \in \mathbb{C}^{d} \times \mathbb{Z}^{d+1}$. The integral $j_{\boldsymbol{v}, \boldsymbol{m}}(x)$ defined above by 6.13) absolutely converges if $\boldsymbol{v} \in \bigcup_{a \in\left[-\frac{1}{2},\left|m_{d+1}\right|\right]} \mathbb{S}^{d}\left(\frac{1}{2}\left(-\frac{1}{2}-a\right) \boldsymbol{e}^{d}, \frac{1}{2}\left(\left\|\boldsymbol{m}^{d}\right\|-a \boldsymbol{e}^{d}\right)\right)$, with $\boldsymbol{m}^{d}=$ $\left(m_{1}, \ldots, m_{d}\right)$ and $\left\|\boldsymbol{m}^{d}\right\|=\left(\left|m_{1}\right|, \ldots,\left|m_{d}\right|\right)$.

To show this, we first recollect some well-known facts concerning $J_{m}(x)$, as $j_{(0, m)}(x)=$ $2 \pi i^{m} J_{m}(4 \pi x)$ in view of (4.4). (1)])

Firstly, for $m \in \mathbb{N}$, we have the Poisson-Lommel integral representation (see [Wat 3.3

$$
J_{m}(x)=\frac{\left(\frac{1}{2} x\right)^{m}}{\Gamma\left(m+\frac{1}{2}\right) \Gamma\left(\frac{1}{2}\right)} \int_{0}^{\pi} \cos (x \cos \theta) \sin ^{2 m} \theta d \theta
$$

This yields the bound

$$
\left|J_{m}(x)\right| \leqslant \frac{\sqrt{\pi}\left(\frac{1}{2} x\right)^{|m|}}{\Gamma\left(|m|+\frac{1}{2}\right)}
$$

for $m \in \mathbb{Z}$. Secondly, the asymptotic expansion of $J_{m}(x)$ (see [Wat $\left.7.21(1)\right]$ ) provides the estimate

$$
J_{m}(x) \ll_{m} x^{-\frac{1}{2}} .
$$

Combining these, we then arrive at the following lemma.

LEMMA 6.6. Let $m$ be an integer.

(1). We have the estimates

$$
j_{(0, m)}(x) \ll_{m} x^{|m|}, \quad j_{(0, m)}(x) \ll_{m} x^{-\frac{1}{2}} .
$$

(2). More generally, for any $a \in\left[-\frac{1}{2},|m|\right]$, we have the estimate

$$
j_{(0, m)}(x) \ll_{m} x^{a}
$$

Proof of Proposition 6.5 We divide $\mathbb{R}_{+}=(0, \infty)$ into the union of two intervals, $I_{-} \cup I_{+}=(0,1] \cup[1, \infty)$. Accordingly, the integral in 6.13 is partitioned into $2^{d}$ many integrals, each of which is supported on some hyper-cube $I_{\varrho}=I_{\varrho_{1}} \times \ldots \times I_{\varrho_{d}}$ for $\varrho \in\{+,-\}^{d}$. For each such integral, we estimate $j_{\left(0, m_{l}\right)}\left(x y_{l}^{-1}\right)$ using the first or the second estimate in Lemma 6.6 (1) according as $\varrho_{l}=+$ or $\varrho_{l}=-$ and apply the bound in Lemma 6.6 (2) for $j_{\left(0, m_{d+1}\right)}\left(x y_{1} \ldots y_{d}\right)$. In this way, for any $a \in\left[-\frac{1}{2},\left|m_{d+1}\right|\right]$, we have

$$
2^{d} \int_{\mathbb{R}_{+}^{d}}\left|j_{\left(0, m_{d+1}\right)}\left(x y_{1} \ldots y_{d}\right)\right| \prod_{l=1}^{d}\left|y_{l}^{2 v_{l}-1} j_{\left(0, m_{l}\right)}\left(x y_{l}^{-1}\right)\right| d y_{d} \ldots d y_{1}
$$




$$
\ll \sum_{\varrho \in\{+,-\}^{d}} x^{\sum_{l \in L_{+}(\varrho)}\left|m_{l}\right|-\frac{1}{2}\left|L_{-}(\varrho)\right|+a} I_{2 \boldsymbol{v}+a e^{d}, \boldsymbol{m}^{d}}(\varrho),
$$

with the auxiliary definition

$$
I_{\lambda, \boldsymbol{k}}(\varrho)=\int_{I_{\varrho}}\left(\prod_{l \in L_{+}(\varrho)} y_{l}^{\Re \mathrm{R} \lambda_{l}-\left|k_{l}\right|-1}\right)\left(\prod_{l \in L_{-}(\varrho)} y_{l}^{\Re \mathrm{R} \lambda_{l}-\frac{1}{2}}\right) d y_{d} \ldots d y_{1}, \quad(\lambda, \boldsymbol{k}) \in \mathbb{C}^{d} \times \mathbb{Z}^{d},
$$

and $L_{ \pm}(\varrho)=\left\{l: \varrho_{l}= \pm\right\}$. The implied constant depends only on $\boldsymbol{m}$ and $d$. It is clear that all the integrals $I_{2 v+a e^{d}, \boldsymbol{m}^{d}}(\varrho)$ absolutely converge if $-\frac{1}{2}<2 \Re \mathfrak{R e} v_{l}+a<\left|m_{l}\right|$ for all $l=1, \ldots, d$. The proof is then completed.

Q.E.D.

Remark 6.7. When $d=1$, one may apply the two estimates in Lemma 6.6 (1) to $j_{\left(0, m_{2}\right)}(x y)$ in the similar fashion as $j_{\left(0, m_{1}\right)}\left(x y^{-1}\right)$. Then

$$
\begin{aligned}
2 \int_{0}^{\infty} \mid y^{2 v-1} j_{\left(0, m_{1}\right)} & \left(x y^{-1}\right) j_{\left(0, m_{2}\right)}(x y) \mid d y \\
& \ll_{m_{1}, m_{2}} x^{\left|m_{1}\right|-\frac{1}{2}} \int_{1}^{\infty} y^{2 \Re \mathrm{Re} v-\left|m_{1}\right|-\frac{3}{2}} d y+x^{\left|m_{2}\right|-\frac{1}{2}} \int_{0}^{1} y^{2 \Re \mathrm{Re} v+\left|m_{2}\right|-\frac{1}{2}} d y .
\end{aligned}
$$

Since both integrals above absolutely converge if $-\left|m_{2}\right|-\frac{1}{2}<2 \Re \mathrm{e} v<\left|m_{1}\right|+\frac{1}{2}$, this also proves Proposition 6.5 in the case $d=1$.

6.5.2. Equality between $j_{(\mu, m)}(x)$ and $j_{v, m}\left(x^{\frac{1}{d+1}}\right)$.

Proposition 6.8. Let $(\boldsymbol{v}, \boldsymbol{m}) \in \mathbb{C}^{d} \times \mathbb{Z}^{d+1}$ be as in Proposition 6.5 so that the integral $j_{v, \boldsymbol{m}}(x)$ absolutely converges. Suppose that $\boldsymbol{\mu} \in \mathbb{L}^{d}$ and $\boldsymbol{v} \in \mathbb{C}^{d}$ satisfy the relations given in Notation 6.1. Then we have the identity

$$
j_{(\mu, \boldsymbol{m})}(x)=j_{v, \boldsymbol{m}}\left(x^{\frac{1}{d+1}}\right) .
$$

PROoF. Some change of variables turns the integral in Corollary 5.14 into

$$
2^{d+1} e^{i m \phi} \int_{\mathbb{R}_{+}^{d+1}} \varphi(y) j_{\left(0, m_{d+1}\right)}\left((x y)^{\frac{1}{d+1}} y_{1} \ldots y_{d}\right) \prod_{l=1}^{d} y_{l}^{2 v_{l}-1} j_{\left(0, m_{l}\right)}\left((x y)^{\frac{1}{d+1}} y_{l}^{-1}\right) y d y d y_{d} \ldots d y_{1} .
$$

Corollary 3.16 and 5.14 along with the second formula in 6.2 , yield

$$
\begin{aligned}
& 2 \int_{\mathbb{R}_{+}} \varphi(y) j_{(\mu, \boldsymbol{m})}(x y) y d y= \\
& 2^{d+1} \int_{\mathbb{R}_{+}^{d+1}} \varphi(y) j_{\left(0, m_{d+1}\right)}\left((x y)^{\frac{1}{d+1}} y_{1} \ldots y_{d}\right) \prod_{l=1}^{d} y_{l}^{2 v_{l}-1} j_{\left(0, m_{l}\right)}\left((x y)^{\frac{1}{d+1}} y_{l}^{-1}\right) y d y d y_{d} \ldots d y_{1},
\end{aligned}
$$

for any $\varphi \in \mathscr{S}\left(\mathbb{R}_{+}\right)$, provided that $\mathfrak{R e} \mu_{1}>\ldots>\mathfrak{R e} \mu_{d}>\mathfrak{R e} \mu_{d+1}$ or equivalently $\mathfrak{R e} v_{1}>\ldots>\mathfrak{R e} v_{d}>0$. In view of Proposition 6.5, the integral on the right hand side is absolute convergent at least when $\frac{1}{4}>\mathfrak{R e} v_{1}>\ldots>\mathfrak{R e} v_{d}>0$. Therefore, the asserted equality holds on the domain $\left\{\boldsymbol{v} \in \mathbb{C}^{d}: \frac{1}{4}>\mathfrak{R e} v_{1}>\ldots>\Re \mathrm{Re} v_{d}>0\right\}$ and remains valid on the whole domain of convergence for $j_{v, m}(x)$ given in Proposition 6.5 due to the principle of analytic continuation.

Q.E.D. 


\subsubsection{An Auxiliary Lemma.}

Lemma 6.9. Let $(\boldsymbol{v}, \boldsymbol{m}) \in \mathbb{C}^{d} \times \mathbb{Z}^{d+1}$ and $m \in \mathbb{Z}$. Set $A=\max _{l=1, \ldots, d+1}\left\{\left|m_{l}\right|\right\}$. Suppose $\boldsymbol{v} \in \bigcup_{a \in\left[-\frac{1}{2}, 0\right]} \mathbb{S}^{d}\left(-\frac{1}{4}-\frac{1}{2} a,-\frac{1}{2} a\right)$. We have the estimate

$$
\begin{gathered}
2^{d} \int_{\mathbb{R}_{+}^{d}}\left|j_{\left(0, m_{d+1}+m\right)}\left(x y_{1} \ldots y_{d}\right)\right| \prod_{l=1}^{d}\left|y_{l}^{2 v_{l}-1} j_{\left(0, m_{l}+m\right)}\left(x y_{l}^{-1}\right)\right| d y_{d} \ldots d y_{1} \\
\ll_{\boldsymbol{m}, d} \sum_{\varrho \neq \varrho^{-}}\left(\frac{2 \pi e x}{|m|+1}\right)^{\left|L_{+}(\varrho)\right||m|}(|m|+1)^{2\left|L_{-}(\varrho)\right|+A\left|L_{+}(\varrho)\right|} \\
x^{-\frac{1}{2}\left|L_{-}(\varrho)\right|} \max \left\{x^{\left|L_{+}(\varrho)\right| A}, x^{-\left|L_{+}(\varrho)\right| A-\frac{1}{2}}\right\} \\
+\left(\frac{2 \pi e x}{|m|+1}\right)^{|m|}(|m|+1)^{A} x^{-\frac{d}{2}} \max \left\{x^{A}, x^{-A}\right\},
\end{gathered}
$$

where $\varrho \in\{+,-\}^{d}, \varrho_{-}=(-, \ldots,-)$ and $L_{ \pm}(\varrho)=\left\{l: \varrho_{l}= \pm\right\}$.

Firstly, we require the bound 6.14) for $J_{m}(x)$. Secondly, we observe that when $x \geqslant$ $(|m|+1)^{2}$ the bound 6.15 for $J_{m}(x)$ can be improved so that the implied constant becomes absolute. This follows from the asymptotic expansion of $J_{m}(x)$ given in [Olv3, §7.13.1]. Moreover, we have Bessel's integral representation (see [Wat 2.2 (1)])

$$
J_{m}(x)=\frac{1}{2 \pi} \int_{0}^{2 \pi} \cos (m \theta-x \sin \theta) d \theta,
$$

which yields the bound

$$
\left|J_{m}(x)\right| \leqslant 1
$$

We then have the following lemma (compare [HM, Proposition 8]).

LeMma 6.10. Let $m$ be an integer.

(1). The following two estimates hold

$$
j_{(0, m)}(x) \ll \frac{(2 \pi x)^{|m|}}{\Gamma\left(|m|+\frac{1}{2}\right)}, \quad j_{(0, m)}(x) \ll \frac{|m|+1}{\sqrt{x}},
$$

with absolute implied constants.

(2). For any $a \in\left[-\frac{1}{2}, 0\right]$ we have the estimate

$$
j_{(0, m)}(x) \ll\left((|m|+1)^{-2} x\right)^{a}
$$

with absolute implied constant.

Proof of Lemma 6.9. Our proof here is similar to that of Proposition 6.5, except that

- Lemma 6.10(1) and (2) are applied in place of Lemma 6.6(1) and (2) respectively to bound $j_{\left(0, m_{l}+m\right)}\left(x y_{l}^{-1}\right)$ and $j_{\left(0, m_{d+1}+m\right)}\left(x y_{1} \ldots y_{d}\right)$, and

- the first estimate in Lemma 6.10 (1) is used for $j_{\left(0, m_{d+1}+m\right)}\left(x y_{1} \ldots y_{d}\right)$ in the case $\varrho=\varrho_{-}$.

In this way, one obtains the following estimate

$$
2^{d} \int_{\mathbb{R}_{+}^{d}}\left|j_{\left(0, m_{d+1}+m\right)}\left(x y_{1} \ldots y_{d}\right)\right| \prod_{l=1}^{d}\left|y_{l}^{2 v_{l}-1} j_{\left(0, m_{l}+m\right)}\left(x y_{l}^{-1}\right)\right| d y_{d} \ldots d y_{1}
$$




$$
\begin{gathered}
\ll \sum_{\varrho \neq \varrho_{-}} \frac{\prod_{l \in L_{-}(\varrho)}\left(\left|m_{l}+m\right|+1\right)^{1-2 a}}{\prod_{l \in L_{+}(\varrho)} \Gamma\left(\left|m_{l}+m\right|+\frac{1}{2}\right)\left(\left|m_{l}+m\right|+1\right)^{2 a}} \\
+\frac{(2 \pi x)^{\sum_{l \in L_{+}(\varrho)}\left|m_{l}+m\right|-\frac{1}{2}\left|L_{-}(\varrho)\right|+a} I_{2 \boldsymbol{v}+a \boldsymbol{e}^{d}, \boldsymbol{m}^{d}+m \boldsymbol{e}^{d}}(\varrho)}{\Gamma\left(\left|m_{d+1}+m\right|+\frac{1}{2}\right)}(2 \pi x)^{-\frac{d}{2}+\left|m_{d+1}+m\right|} I_{2 \boldsymbol{v}+\left|m_{d+1}+m\right| \boldsymbol{e}^{d}}\left(\varrho_{-}\right),
\end{gathered}
$$

ワwith $a \in\left[-\frac{1}{2}, 0\right]$. Suppose that $-\frac{1}{2}-a<2 \Re \mathrm{Re} v_{l}<-a$ for all $l=1, \ldots, d$, then the integrals $I_{2 v+a e^{d}, \boldsymbol{m}^{d}+m e^{d}}(\varrho)$ and $I_{2 v+\left|m_{d+1}+m\right| e^{d}}\left(\varrho_{-}\right)$are absolutely convergent and of size $O_{d}\left(\prod_{l \in L_{+}(\varrho)}\left(\left|m_{l}+m\right|+1\right)^{-1}\right)$ and $O_{d}\left(\left(\left|m_{d+1}+m\right|+1\right)^{-d}\right)$ respectively. A final estimation using Stirling's asymptotic formula yields our asserted bound.

Q.E.D.

Remark 6.11. In the case $d=1$, modifying over the ideas in Remark 6.7, one may show the slightly improved estimate

$$
\begin{aligned}
2 \int_{0}^{\infty} \mid y^{2 v-1} j_{\left(0, m_{1}+m\right)} & \left(x y^{-1}\right) j_{\left(0, m_{2}+m\right)}(x y) \mid d y \\
& \ll m_{1}, m_{2}\left(\frac{2 \pi e x}{|m|+1}\right)^{|m|}(|m|+1)^{A} x^{-\frac{1}{2}} \max \left\{x^{A}, x^{-A}\right\},
\end{aligned}
$$

given that $|\Re \mathfrak{R} v|<\frac{1}{4}$, with $A=\max \left\{\left|m_{1}\right|,\left|m_{2}\right|\right\}$.

6.6. The Series of Integrals $J_{v, m}(x, u)$. We define the following series of integrals,

$$
\begin{aligned}
& J_{v, \boldsymbol{m}}(x, u)=\frac{1}{2 \pi} \sum_{m \in \mathbb{Z}} u^{m} j_{v, \boldsymbol{m}+m e^{n}}(x) \\
= & \frac{2^{d-1}}{\pi} \sum_{m \in \mathbb{Z}} u^{m} \int_{\mathbb{R}_{+}^{d}} j_{\left(0, m_{d+1}+m\right)}\left(x y_{1} \ldots y_{d}\right) \prod_{l=1}^{d} y_{l}^{2 v_{l}-1} j_{\left(0, m_{l}+m\right)}\left(x y_{l}^{-1}\right) d y_{d} \ldots d y_{1},
\end{aligned}
$$

with $x \in \mathbb{R}_{+}$and $u \in \mathbb{C},|u|=1$.

6.6.1. Absolute Convergence of $J_{v, m}(x, u)$. We have the following direct consequence of Lemma 6.9 .

Proposition 6.12. Let $(\boldsymbol{v}, \boldsymbol{m}) \in \mathbb{C}^{d} \times \mathbb{Z}^{d+1}$. The series of integrals $J_{\boldsymbol{v}, \boldsymbol{m}}(x, u)$ defined by 6.17) is absolutely convergent if $v \in \bigcup_{a \in\left[-\frac{1}{2}, 0\right]} \mathbb{S}^{d}\left(-\frac{1}{4}-\frac{1}{2} a,-\frac{1}{2} a\right)$.

6.6.2. Equality between $J_{(\mu, m)}\left(x e^{i \phi}\right)$ and $J_{v, m}\left(x^{\frac{1}{d+1}}, e^{i \phi}\right)$. In view of Proposition 6.8 along with 3.43) and 6.17), the following proposition is readily established.

Proposition 6.13. Let $(\boldsymbol{v}, \boldsymbol{m}) \in \mathbb{C}^{d} \times \mathbb{Z}^{d+1}$. Suppose that $\boldsymbol{v}$ satisfies the condition in Proposition 6.12 so that $J_{v, m}(x, u)$ is absolutely convergent. Then, given that $\boldsymbol{\mu}$ and $\boldsymbol{v}$ satisfy the relations in Notation 6.1 we have the identity

$$
J_{(\mu, \boldsymbol{m})}\left(x e^{i \phi}\right)=J_{v, \boldsymbol{m}}\left(x^{\frac{1}{d+1}}, e^{i \phi}\right),
$$

with $x \in \mathbb{R}_{+}$and $\phi \in \mathbb{R} / 2 \pi \mathbb{Z}$.

\footnotetext{
$\mathrm{V}_{\text {When } \varrho=} \varrho_{-}, \boldsymbol{k}$ does not occur in the definition of $I_{\lambda, k}\left(\varrho_{-}\right)$and is therefore suppressed from the subscript.
} 


\subsection{Proof of Theorem 6.3.}

Lemma 6.14. Let $\boldsymbol{k} \in \mathbb{Z}^{d}$ and recall the integral $J_{\boldsymbol{k}}(\boldsymbol{y} ; x, \phi)$ defined by 6.9 6.10. We have the following absolutely convergent series expansion of $J_{\boldsymbol{k}}(\boldsymbol{y} ; x, \phi)$

$$
J_{\boldsymbol{k}}(\boldsymbol{y} ; 2 \pi x, \phi)=\frac{1}{2 \pi} \sum_{m \in \mathbb{Z}} e^{i m \phi} j_{(0, m)}\left(x y_{1} \ldots y_{d}\right) \prod_{l=1}^{d} j_{\left(0, k_{l}+m\right)}\left(x y_{l}^{-1}\right) .
$$

Proof. We find in $\$ 4.4$ the integral representation

$$
j_{(0, m)}(x)=\int_{\mathbb{R} / 2 \pi \mathbb{Z}} e^{i m \theta+4 \pi i x \cos \theta} d \theta
$$

as well as the Fourier series expansion

$$
e^{4 \pi i x \cos \phi}=\frac{1}{2 \pi} \sum_{m \in \mathbb{Z}} j_{(0, m)}(x) e^{i m \phi} .
$$

Therefore

$$
\begin{aligned}
& \frac{1}{2 \pi} \sum_{m \in \mathbb{Z}} e^{i m \phi} j_{(0, m)}\left(x y_{1} \ldots y_{d}\right) \prod_{l=1}^{d} j_{\left(0, k_{l}+m\right)}\left(x y_{l}^{-1}\right) \\
= & \frac{1}{2 \pi} \sum_{m \in \mathbb{Z}} e^{i m \phi} j_{(0, m)}\left(x y_{1} \ldots y_{d}\right) \int_{(\mathbb{R} / 2 \pi \mathbb{Z})^{d}} e^{i m \sum_{l=1}^{d} \theta_{l}} e^{i \sum_{l=1}^{d}\left(i k_{l} \theta+4 \pi i x y_{l}^{-1} \cos \theta_{l}\right)} d \theta_{d} \ldots d \theta_{1} \\
= & \int_{(\mathbb{R} / 2 \pi \mathbb{Z})^{d}}\left(\frac{1}{2 \pi} \sum_{m \in \mathbb{Z}} e^{i m\left(\sum_{l=1}^{d} \theta_{l}+\phi\right)} j_{(0, m)}\left(x y_{1} \ldots y_{d}\right)\right) e^{i \sum_{l=1}^{d}\left(i k_{l} \theta+4 \pi i x y_{l}^{-1} \cos \theta_{l}\right)} d \theta_{d} \ldots d \theta_{1} \\
= & \int_{(\mathbb{R} / 2 \pi \mathbb{Z})^{d}} e^{4 \pi i x y_{1} \ldots y_{d} \cos \left(\sum_{l=1}^{d} \theta_{l}+\phi\right)} e^{i \sum_{l=1}^{d}\left(i k_{l} \theta+4 \pi i x y_{l}^{-1} \cos \theta_{l}\right)} d \theta_{d} \ldots d \theta_{1} .
\end{aligned}
$$

The absolute convergence required for the validity of each equality above is justified by the first estimate for $j_{(0, m)}(x)$ in Lemma 6.10 (1). The proof is completed, since the last line is exactly the definition of $J_{\boldsymbol{k}}(\boldsymbol{y} ; 2 \pi x, \phi)$.

Q.E.D.

Inserting the series expansion of $J_{k}(y ; 2 \pi x, \phi)$ in Lemma 6.14 into the integral in (6.12) and interchanging the order of integration and summation, one arrives exactly at the series of integrals $J_{v,(k, 0)}\left(x, e^{i \phi}\right)=e^{-i m_{d+1} \phi} J_{v, \boldsymbol{m}}\left(x, e^{i \phi}\right)$. The first assertion on absolute convergence in Theorem 6.3 follows immediately from Proposition 6.12, whereas the identity in the second assertion is a direct consequence of Proposition 6.13

\subsection{The Rank-Two Case $(d=1)$.}

6.8.1. The Case of Bessel Functions. When $x>0$ and $|\Re e v|<1$, we have the following integral representations of Bessel functions due to Mehler and Sonine ([Wat $6.21(10,11), 6.22(13)])$

$$
\begin{aligned}
H_{v}^{(1,2)}(x) & = \pm \frac{2 e^{\mp \frac{1}{2} \pi i v}}{\pi i} \int_{0}^{\infty} e^{ \pm i x \cosh r} \cosh (v r) d r \\
K_{v}(x) & =\frac{1}{\cos \left(\frac{1}{2} \pi v\right)} \int_{0}^{\infty} \cos (x \sinh r) \cosh (v r) d r .
\end{aligned}
$$

The change of variables $t=e^{r}$ yields

$$
\pm \pi i e^{ \pm \frac{1}{2} \pi i v} H_{v}^{(1,2)}(2 x)=\int_{0}^{\infty} t^{v-1} e^{ \pm i x\left(t+t^{-1}\right)} d t
$$




$$
2 e^{ \pm \frac{1}{2} \pi i v} K_{v}(2 x)=\int_{0}^{\infty} t^{v-1} e^{ \pm i x\left(t-t^{-1}\right)} d t
$$

The integrals in these formulae are exactly the formal integrals in 6.4 in the case $d=1$. They conditionally converge if $|\Re \mathrm{e} v|<1$, but diverge if otherwise.

6.8.2. The Real Case. By the formula (6.6), the formal integral $J_{v, \epsilon}(2 \pi \sqrt{x}, \pm)$, which represents the Bessel kernel $J_{\left(\frac{1}{2} v,-\frac{1}{2} v\right),(\epsilon, 0)}( \pm x)$, is reduced to the integrals in $\$ 6.8 .1$.

\subsubsection{The Complex Case.}

Lemma 6.15. Let $k \in \mathbb{Z}$. Recall from 6.9, 6.10) the definition

$$
J_{k}(y ; x, \phi)=\int_{0}^{2 \pi} e^{i k \theta+2 i x y^{-1} \cos \theta+2 i x y \cos (\theta+\phi)} d \theta, \quad x, y \in(0, \infty), \phi \in[0,2 \pi) .
$$

Define $Y(y, \phi)=\left|y^{-1}+y e^{i \phi}\right|=\sqrt{y^{-2}+2 \cos \phi+y^{2}}, \Phi(y, \phi)=\arg \left(y^{-1}+y e^{i \phi}\right)$ and $E(y, \phi)=e^{i \Phi(y, \phi)}$. Then

$$
J_{k}(y ; x, \phi)=2 \pi i^{k} E(y, \phi)^{-k} J_{k}(2 x Y(y, \phi)) .
$$

Proof. 6.19 follows immediately from the identity

$$
2 \pi i^{k} J_{k}(x)=\int_{0}^{2 \pi} e^{i k \theta+i x \cos \theta} d \theta
$$

along with the observation

$$
y^{-1} \cos \theta+y \cos (\theta+\phi)=\mathfrak{R e}\left(y^{-1} e^{i \theta}+y e^{i(\theta+\phi)}\right)=Y(y, \phi) \cos (\theta+\Phi(y, \phi)) .
$$

Q.E.D.

Proposition 6.16. Let $v \in \mathbb{C}$ and $k \in \mathbb{Z}$. Recall the definition of $J_{v, k}\left(x, e^{i \phi}\right)$ given by 6.12. Then

$$
J_{v, k}\left(x, e^{i \phi}\right)=4 \pi i^{k} \int_{0}^{\infty} y^{2 v-1}\left[y^{-1}+y e^{i \phi}\right]^{-k} J_{k}\left(2 x\left|y^{-1}+y e^{i \phi}\right|\right) d y,
$$

with $x \in(0, \infty)$ and $\phi \in[0,2 \pi)$. Here, we recall the notation $[z]=z /|z|$. The integral in 6.20) converges when $|\mathfrak{R e} v|<\frac{3}{4}$ and the convergence is absolute if and only if $|\mathfrak{R e} v|<\frac{1}{4}$. Moreover, it is analytic with respect to $v$ on the open vertical strip $\mathbb{S}\left(-\frac{3}{4}, \frac{3}{4}\right)$.

Proof. 6.20 follows immediately from Lemma 6.15.

As for the convergence, since one arrives at an integral of the same form with $v, \phi$ replaced by $-v,-\phi$ if the variable is changed from $y$ to $y^{-1}$, it suffices to consider the integral

$$
\int_{2}^{\infty} y^{2 v-1} e^{-i k \Phi(y, \phi)} J_{k}(2 x Y(y, \phi)) d y
$$

for $\Re$ e $v<\frac{3}{4}$. We have the following asymptotic of $J_{k}(x)$ (see [Wat 7.21 (1)])

$$
J_{k}(x)=\left(\frac{2}{\pi x}\right)^{\frac{1}{2}} \cos \left(x-\frac{1}{2} k \pi-\frac{1}{4} \pi\right)+O_{k}\left(x^{-\frac{3}{2}}\right) .
$$

The error term contributes an absolutely convergent integral when $\Re \mathrm{e} v<\frac{3}{4}$, whereas the integral coming from the main term absolutely converges if and only if $\Re e v<\frac{1}{4}$. We are now reduced to the integral

$$
\int_{2}^{\infty} y^{2 v-1} e^{-i k \Phi(y, \phi)}(x Y(y, \phi))^{-\frac{1}{2}} e^{ \pm 2 i x Y(y, \phi)} d y
$$


In order to see the convergence, we split out $e^{ \pm 2 i x y}$ from $e^{ \pm 2 i x Y(y, \phi)}$ and put $f_{v, k}(y ; x, \phi)=$ $y^{2 v-1} e^{-i k \Phi(y, \phi)}(x Y(y, \phi))^{-\frac{1}{2}} e^{ \pm 2 i x(Y(y, \phi)-y)}$. Partial integration turns the above integral into

$$
\mp \frac{1}{2 i x^{\frac{3}{2}}}\left(2^{2 v-1} e^{-i k \Phi(2, \phi)} Y(2, \phi)^{-\frac{1}{2}} e^{ \pm 2 i x Y(2, \phi)}+\int_{2}^{\infty}\left(\partial f_{v, k} / \partial y\right)(y ; x, \phi) e^{ \pm 2 i x y} d y\right) .
$$

Some calculations show that $\left(\partial f_{v, k} / \partial y\right)(y ; x, \phi) \ll_{v, k, x} y^{2 \Re \mathrm{Re} v-\frac{5}{2}}$ for $y \geqslant 2$, and hence the integral in the second term is absolutely convergent when $\operatorname{Re} v<\frac{3}{4}$. With the above arguments, the analyticity with respect to $v$ is obvious.

Q.E.D.

Corollary 6.17. Let $\mu \in \mathbb{S}\left(-\frac{3}{8}, \frac{3}{8}\right)$ and $m \in \mathbb{Z}$. We have

$$
J_{(\mu,-\mu, m, 0)}\left(x e^{i \phi}\right)=4 \pi i^{m} \int_{0}^{\infty} y^{4 \mu-1}\left[y^{-1}+y e^{i \phi}\right]^{-m} J_{m}\left(4 \pi \sqrt{x}\left|y^{-1}+y e^{i \phi}\right|\right) d y,
$$

with $x \in(0, \infty)$ and $\phi \in[0,2 \pi)$. The integral in 6.21] converges if $|\Re \mathrm{e} \mu|<\frac{3}{8}$ and absolutely converges if and only if $|\mathfrak{R e} \mu|<\frac{1}{8}$.

Proof. From Theorem 6.3 we see that 6.21 holds for $\mathbb{S}\left(-\frac{1}{8}, \frac{1}{8}\right)$. In view of Proposition 6.16 the right hand side of 6.21) is analytic in $\mu$ on $\mathbb{S}\left(-\frac{3}{8}, \frac{3}{8}\right)$, and therefore it is allowed to extend the domain of equality from $\mathbb{S}\left(-\frac{1}{8}, \frac{1}{8}\right)$ onto $\mathbb{S}\left(-\frac{3}{8}, \frac{3}{8}\right)$.

Q.E.D.

\section{A. A Prototypical Example of Bessel Functions}

Before delving into the general analytic theory of Bessel functions in Chapter 2, we shall give here a very typical example of $J(x ; \boldsymbol{\varsigma}, \lambda)$ which illustrates their asymptotic nature with dependence on $\boldsymbol{s}$.

When $n=1$, we simply have two oscillatory exponential functions

$$
J(x ; \pm, 0)=e^{ \pm i x} .
$$

We then consider the case when $n=2$. According to [Wat 3.4 (3, 6), 3.71 (13)],

$$
J_{\frac{1}{2}}(x)=\left(\frac{2}{\pi x}\right)^{\frac{1}{2}} \sin x, \quad J_{-\frac{1}{2}}(x)=\left(\frac{2}{\pi x}\right)^{\frac{1}{2}} \cos x .
$$

The connection formulae in $[0.5)$ ([Wat $3.61(5,6)]$ ) imply that

$$
H_{\frac{1}{2}}^{(1)}(x)=-i\left(\frac{2}{\pi x}\right)^{\frac{1}{2}} e^{i x}, \quad H_{\frac{1}{2}}^{(2)}(x)=i\left(\frac{2}{\pi x}\right)^{\frac{1}{2}} e^{-i x}
$$

Moreover, [Wat 3.71 (13)] reads

$$
K_{\frac{1}{2}}(x)=\left(\frac{\pi}{2 x}\right)^{\frac{1}{2}} e^{-x} .
$$

Therefore, the formulae in Proposition 4.4 yield

$$
J\left(x ; \pm, \pm, \frac{1}{4},-\frac{1}{4}\right)=\left(\frac{\pi}{x}\right)^{\frac{1}{2}} e^{ \pm 2 i x \pm \frac{1}{4} \pi i}, \quad J\left(x ; \pm, \mp, \frac{1}{4},-\frac{1}{4}\right)=\left(\frac{\pi}{x}\right)^{\frac{1}{2}} e^{-2 x \mp \frac{1}{4} \pi i} .
$$

We now prove that these formulae admit generalizations to arbitrary rank.

Proposition A.1. For $\boldsymbol{s} \in\{+,-\}^{n}$ we define $L_{ \pm}(\boldsymbol{s})=\left\{l: \varsigma_{l}= \pm\right\}$ and $n_{ \pm}(\boldsymbol{s})=$ $\left|L_{ \pm}(\boldsymbol{\varsigma})\right|$. Put $\xi(\boldsymbol{\varsigma})=i e^{\pi i \frac{n_{-}(\boldsymbol{s})-n_{+}(\boldsymbol{s})}{2 n}}=\mp e^{\mp \pi i \frac{n_{ \pm}(\boldsymbol{s})}{n}}$. Suppose $\lambda=\frac{1}{n}\left(\frac{n-1}{2}, \ldots,-\frac{n-1}{2}\right)$. Then

$$
J(x ; \boldsymbol{\varsigma}, \lambda)=\frac{c(\boldsymbol{\varsigma})}{\sqrt{n}}\left(\frac{2 \pi}{x}\right)^{\frac{n-1}{2}} e^{i n \xi(\boldsymbol{\varsigma}) x}
$$


with $c(\boldsymbol{S})=e\left(\mp \frac{n-1}{8} \mp \frac{n_{ \pm}(\boldsymbol{S})}{2 n} \pm \frac{1}{2 n} \sum_{l \in L_{ \pm}(\boldsymbol{S})} l\right)$.

Proof. Using the multiplication formula of the Gamma function

$$
\prod_{k=0}^{n-1} \Gamma\left(s+\frac{k}{n}\right)=(2 \pi)^{\frac{n-1}{2}} n^{\frac{1}{2}-n s} \Gamma(n s)
$$

straightforward calculations yield

$$
G(s ; \boldsymbol{S}, \lambda)=c_{1}(\boldsymbol{S})(2 \pi)^{\frac{n-1}{2}} n^{\frac{1}{2}-n\left(s-\frac{n-1}{2 n}\right)} \Gamma\left(n\left(s-\frac{n-1}{2 n}\right)\right) e\left(\frac{n_{+}(\boldsymbol{\boldsymbol { S }})-n_{-}(\boldsymbol{\boldsymbol { S }})}{4} \cdot s\right),
$$

with $c_{1}(\boldsymbol{S})=e\left(\mp \frac{(n+1) n_{ \pm}(\boldsymbol{s})}{4 n} \pm \frac{1}{2 n} \sum_{l \in L_{ \pm}(\boldsymbol{s})} l\right)$. Inserting this into the contour integral in (3.6) and making the change of variables from $s$ to $\frac{1}{n}\left(s+\frac{n-1}{2}\right)$, one arrives at

$$
J(x ; \boldsymbol{\varsigma}, \lambda)=\frac{c_{1}(\boldsymbol{\varsigma}) c_{2}(\boldsymbol{s})}{\sqrt{n}}\left(\frac{2 \pi}{x}\right)^{\frac{n-1}{2}} \frac{1}{2 \pi i} \int_{n \mathrm{e}_{-\frac{n-1}{2}}} \Gamma(s) e\left(\frac{n_{+}(\boldsymbol{\boldsymbol { S }})-n_{-}(\boldsymbol{\boldsymbol { s }})}{4 n} \cdot s\right)(n x)^{-s} d s,
$$

with $c_{2}(\boldsymbol{S})=e\left(\mp \frac{n-1}{8} \pm \frac{(n-1) n_{ \pm}(\boldsymbol{s})}{4 n}\right)$. A.1 now follows from (4.3) if the contour $\mathcal{C}$ is suitably chosen. 



\section{CHAPTER 2}

\section{Analytic Theory of Bessel Functions}

This chapter is on the analytic theory of Bessel functions. There are two parts of this theory, formal integrals and Bessel differential equations. Combining these, this chapter culminates at the connection formulae for Bessel functions.

In \$7, 8 and 9 we shall first rigorously justify the formal integral $J_{v}(x ; \boldsymbol{s})$ as a representation of the Bessel function $J(x ; \lambda, \boldsymbol{\varsigma})$ and then use the stationary phase method of Hörmander for the oscillatory multiple integral $J_{v}(x ; \boldsymbol{\varsigma})$ to study the asymptotic of $J(x ; \lambda, \boldsymbol{\varsigma})$ on $\mathbb{R}_{+}$or its analytic continuation onto the upper or lower half plane $\mathbb{H}^{ \pm}$.

In $\$ 10$ we shall derive the differential equation, namely Bessel equation, satisfied by the Bessel function $J(x ; \boldsymbol{s}, \lambda)$.

In $\$ 11$ we shall study two types of Bessel functions, $J_{l}(z ; \varsigma, \lambda)$ and $J(z ; \lambda ; \xi)$, arising as solutions of Bessel equations in accordance with their asymptotics at zero and infinity respectively.

In $\$ 12$, using the asymptotic theory we have developed in previous sections, we shall present various connection formulae that relate Bessel functions $J(z ; \boldsymbol{\varsigma}, \lambda), J_{l}(z ; \varsigma, \lambda)$ and $J(z ; \lambda ; \xi)$.

In $\$ 13$, using results from $\$ 11$, we shall make improvements on the asymptotic of $J(x ; \lambda, \boldsymbol{\varsigma})$ obtained in $\$ 9$

In Appendix B for the purpose of comparison, we shall include the approach to the asymptotics of Bessel functions from the literature using Stirling's asymptotic formula for the Gamma function.

\section{The Rigorous Interpretation of the Formal Integral $J_{v}(x ; \varsigma)$}

We first introduce some notations. Let $d$ be a positive integer, $\boldsymbol{t}=\left(t_{1}, \ldots, t_{d}\right) \in \mathbb{R}_{+}^{d}$, $\boldsymbol{v}=\left(v_{1}, \ldots, v_{d}\right) \in \mathbb{C}^{d}$ and $\boldsymbol{s}=\left(\varsigma_{1}, \ldots, \varsigma_{d}, \varsigma_{d+1}\right) \in\{+,-\}^{d+1}$. For $a>0$ define $\mathbb{S}_{a}^{d}=\{\boldsymbol{v} \in$ $\mathbb{C}^{d}:\left|\mathfrak{R e} v_{l}\right|<a$ for all $\left.l=1, \ldots, d\right\}$. Denote by $p_{v}$ the power function

$$
p_{v}(\boldsymbol{t})=\prod_{l=1}^{d} t_{l}^{\nu_{l}-1}
$$

and let

$$
\theta(\boldsymbol{t} ; \boldsymbol{s})=\varsigma_{d+1} t_{1} \ldots t_{d}+\sum_{l=1}^{d} \varsigma_{l} t_{l}^{-1},
$$

then the formal integral $J_{v}(x ; \boldsymbol{\varsigma})$ given in 6.4 , which symbolically represents $J(x ; \boldsymbol{\varsigma}, \lambda)$, may be written as

$$
J_{v}(x ; \boldsymbol{S})=\int_{\mathbb{R}_{+}^{d}} p_{\boldsymbol{v}}(\boldsymbol{t}) e^{i x \theta(t ; \boldsymbol{s})} d \boldsymbol{t} .
$$

For $d=1$, it is seen in $\$ 6.8 .1$ that $J_{v}(x ; \boldsymbol{s})$ is conditionally convergent if and only if $|\Re \mathrm{e} v|<1$ but fails to be absolutely convergent. When $d \geqslant 2$, we are in a worse scenario. 
The notion of convergence for multiple integrals is always in the absolute sense. Thus, the $d$-dimensional multiple integral in (7.1) alone does not make any sense, since it is clearly not absolutely convergent.

In the following, we shall address this fundamental convergence issue of the formal integral $J_{v}(x ; \boldsymbol{s})$, relying on its structural simplicity, so that it will be provided with mathematically rigorous meaning $\mathbb{\pi}$ Moreover, it will be shown that our rigorous interpretation of $J_{v}(x ; \boldsymbol{\varsigma})$ is a smooth function of $x$ on $\mathbb{R}_{+}$as well as an analytic function of $v$ on $\mathbb{C}^{d}$.

7.1. Formal Partial Integration Operators. The most crucial observation is that there are two kinds of formal partial integrations. The first kind arises from

$$
\partial\left(e^{\varsigma_{l} i x t_{l}^{-1}}\right)=-\varsigma_{l} i x t_{l}^{-2} e^{\varsigma_{l} i x t_{l}^{-1}} \partial t_{l}
$$

and the second kind from

$$
\partial\left(e^{\varsigma_{d+1} i x t_{1} \ldots t_{d}}\right)=\varsigma_{d+1} i x t_{1} \ldots \widehat{t}_{l} \ldots t_{d} e^{\varsigma_{d+1} i x t_{1} \ldots t_{d}} \partial t_{l}
$$

where $\widehat{t}_{l}$ means that $t_{l}$ is omitted from the product.

Definition 7.1. Let

$$
\mathscr{T}\left(\mathbb{R}_{+}\right)=\left\{h \in C^{\infty}\left(\mathbb{R}_{+}\right): t^{\alpha} h^{(\alpha)}(t) \ll{ }_{\alpha} 1 \text { for all } \alpha \in \mathbb{N}\right\} .
$$

For $h(\boldsymbol{t}) \in \bigotimes^{d} \mathscr{T}\left(\mathbb{R}_{+}\right)$, in the sense that $h(\boldsymbol{t})$ is a linear combination of functions of the form $\prod_{l=1}^{d} h_{l}\left(t_{l}\right)$, define the integral

$$
J_{v}(x ; \boldsymbol{\varsigma} ; h)=\int_{\mathbb{R}_{+}^{d}} h(\boldsymbol{t}) p_{v}(\boldsymbol{t}) e^{i x \theta(\boldsymbol{t} ; \boldsymbol{\varsigma})} d \boldsymbol{t} .
$$

We call $J_{v}(x ; \boldsymbol{\varsigma} ; h)$ a $J$-integral of index $\boldsymbol{v}$. Let us introduce an auxiliary space

$$
\mathscr{J}_{\boldsymbol{v}}(\boldsymbol{\boldsymbol { c }})=\operatorname{Span}_{\mathbb{C}\left[x^{-1}\right]}\left\{J_{\boldsymbol{v}^{\prime}}(x ; \boldsymbol{s} ; h): \boldsymbol{v}^{\prime} \in \boldsymbol{v}+\mathbb{Z}^{d}, h \in \bigotimes^{d} \mathscr{T}\left(\mathbb{R}_{+}\right)\right\} .
$$

Here $\mathbb{C}\left[x^{-1}\right]$ is the ring of polynomials of variable $x^{-1}$ and complex coefficients. Finally, we define $\mathcal{P}_{+, l}$ and $\mathcal{P}_{-, l}$ to be the two $\mathbb{C}\left[x^{-1}\right]$-linear operators on the space $\mathscr{J}_{\boldsymbol{v}}(\boldsymbol{s})$, in symbolic notion, as follows,

$$
\begin{aligned}
& \mathcal{P}_{+, l}\left(J_{v}(x ; \boldsymbol{s} ; h)\right)=\boldsymbol{\varsigma}_{l} \boldsymbol{\varsigma}_{d+1} J_{\boldsymbol{v}+\boldsymbol{e}^{d}+\boldsymbol{e}_{l}}(x ; \boldsymbol{\varsigma} ; h) \\
& -\varsigma_{l} i\left(v_{l}+1\right) x^{-1} J_{v+\boldsymbol{e}_{l}}(x ; \boldsymbol{\varsigma} ; h)-\varsigma_{l} i x^{-1} J_{\boldsymbol{v}+\boldsymbol{e}_{l}}\left(x ; \boldsymbol{\varsigma} ; t_{l} \partial_{l} h\right), \\
& \mathcal{P}_{-, l}\left(J_{v}(x ; \boldsymbol{\varsigma} ; h)\right)=\boldsymbol{\varsigma}_{l} \boldsymbol{\varsigma}_{d+1} J_{\boldsymbol{v}-\boldsymbol{e}^{d}-\boldsymbol{e}_{l}}(x ; \boldsymbol{\varsigma} ; h) \\
& +\varsigma_{d+1} i\left(v_{l}-1\right) x^{-1} J_{v-e^{d}}(x ; \boldsymbol{s} ; h)+\varsigma_{d+1} i x^{-1} J_{v-e^{d}}\left(x ; \boldsymbol{s} ; t_{l} \partial_{l} h\right),
\end{aligned}
$$

where $\boldsymbol{e}_{l}=(\underbrace{0, \ldots, 0,1}_{l}, 0 \ldots, 0)$ and $\boldsymbol{e}^{d}=(1, \ldots, 1)$, and $\partial_{l} h$ is the abbreviated $\partial h / \partial t_{l}$.

The formulations of $\mathcal{P}_{+, l}$ and $\mathcal{P}_{-, l}$ are quite involved at a first glance. However, the most essential feature of these operators is simply index shifts!

Observation 7.2. After the operation of $\mathcal{P}_{+, l}$ on a $J$-integral, all the indices of the three resulting $J$-integrals are nondecreasing and the increment of the $l$-th index is one greater than the others. The operator $\mathcal{P}_{-, l}$ has the effect of decreasing all indices by one except possibly two for the $l$-th index.

\footnotetext{
It turns out that our rigorous interpretation actually coincides with the Hadamard partie finie of the formal integral.
} 

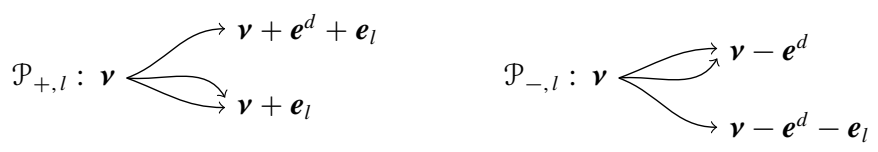

Figure 1. Index Shifts

Lemma 7.3. Let notations be as above.

(1). Let $h(\boldsymbol{t})=\prod_{l=1}^{d} h_{l}\left(t_{l}\right)$. Suppose that the set $\{1,2, \ldots, d\}$ splits into two subsets $L_{+}$ and $L_{-}$such that

- $h_{l}$ vanishes at infinity if $l \in L_{-}$, and

- $h_{l}$ vanishes in a neighbourhood of zero if $l \in L_{+}$.

If $\Re \mathrm{R} v_{l}>0$ for all $l \in L_{-}$and $\mathfrak{R e} v_{l}<0$ for all $l \in L_{+}$, then the $J$-integral $J_{v}(x ; \boldsymbol{s} ; h)$ absolutely converges.

(2). Assume the same conditions in (1). Moreover, suppose that $\Re \mathrm{e} v_{l}>1$ for all $l \in L_{-}$and $\mathfrak{R e} v_{l}<-1$ for all $l \in L_{+}$. Then, for $l \in L_{-}$, all the three J-integrals in the definition of $\mathcal{P}_{+, l}\left(J_{v}(x ; \boldsymbol{s} ; h)\right)$ are absolutely convergent and the operation of $\mathcal{P}_{+, l}$ on $J_{v}(x ; \boldsymbol{s}, h)$ is the actual partial integration of the first kind on the integral over $d t_{l}$. Similarly, for $l \in L_{+}$, the operation of $\mathcal{P}_{-, l}$ preserves absolute convergence and is the actual partial integration of the second kind on the integral over $d t_{l}$.

(3). $\mathcal{P}_{+, l}$ and $\mathcal{P}_{-, l}$ commute with $\mathcal{P}_{+, k}$ and $\mathcal{P}_{-, k}$ if $l \neq k$.

(4). Let $\alpha \in \mathbb{N} . \mathcal{P}_{+, l}^{\alpha}\left(J_{\boldsymbol{v}}(x ; \boldsymbol{\xi} ; h)\right)$ is a linear combination of

$$
\left[v_{l}-1\right]_{\alpha_{3}} x^{-\alpha+\alpha_{1}} J_{v+\alpha_{1} e^{d}+\alpha e_{l}}\left(x ; \boldsymbol{s} ; t_{l}^{\alpha_{2}} \partial_{l}^{\alpha_{2}} h\right),
$$

and $\mathcal{P}_{-, l}^{\alpha}\left(J_{\boldsymbol{v}}(x ; \boldsymbol{\varsigma} ; h)\right)$ is a linear combination of

$$
\left[v_{l}-1\right]_{\alpha_{3}} x^{-\alpha+\alpha_{1}} J_{v-\alpha e^{d}-\alpha_{1} e_{l}}\left(x ; \boldsymbol{s} ; t_{l}^{\alpha_{2}} \partial_{l}^{\alpha_{2}} h\right),
$$

for $\alpha_{1}+\alpha_{2}+\alpha_{3} \leqslant \alpha$. The coefficients of these linear combinations may be uniformly bounded by a constant depending only on $\alpha$.

Proof. (1-3) are obvious. The two statements in (4) follow from calculating

$$
x^{-\alpha} t_{l}^{\alpha+\alpha_{0}} \partial_{l}^{\alpha_{0}}\left(h(\boldsymbol{t}) p_{\boldsymbol{v}}(\boldsymbol{t}) e^{\varsigma_{d+1} i x t_{1} \ldots t_{d}}\right) e^{i x \sum_{k=1}^{d} \varsigma_{k} t_{k}^{-1}}, \quad \alpha_{0} \leqslant \alpha,
$$

and

$$
x^{-\alpha} \partial_{l}^{\alpha}\left(h(\boldsymbol{t}) p_{\nu-\alpha \boldsymbol{e}^{d}+\alpha \boldsymbol{e}_{l}}(\boldsymbol{t}) e^{i x \sum_{k=1}^{d} \varsigma_{k} t_{k}^{-1}}\right) e^{\varsigma_{d+1} i x t_{1} \ldots t_{d}} .
$$

For the latter, one applies the following formula

$$
\frac{d^{\alpha}\left(e^{a t^{-1}}\right)}{d t^{\alpha}}=(-)^{\alpha} \sum_{\beta=1}^{\alpha} \frac{\alpha !(\alpha-1) !}{(\alpha-\beta) ! \beta !(\beta-1) !} a^{\beta} t^{-\alpha-\beta} e^{a t^{-1}}, \quad \alpha \in \mathbb{N}_{+}, a \in \mathbb{C} .
$$

Q.E.D.

7.2. Partitioning the Integral $J_{v}(x ; \boldsymbol{\varsigma})$. Let $I$ be a finite set that includes $\{+,-\}$ and let

$$
\sum_{\varrho \in I} h_{\varrho}(t) \equiv 1, \quad t \in \mathbb{R}_{+},
$$

be a partition of unity on $\mathbb{R}_{+}$such that each $h_{\varrho}$ is a function in $\mathscr{T}\left(\mathbb{R}_{+}\right), h_{-}(t) \equiv 1$ on a neighbourhood of zero and $h_{+}(t) \equiv 1$ for large $t$. Put $h_{\varrho}(t)=\prod_{l=1}^{d} h_{\varrho l}\left(t_{l}\right)$ for $\varrho=$ 
$\left(\varrho_{1}, \ldots, \varrho_{d}\right) \in I^{d}$. We partition the integral $J_{\boldsymbol{v}}(x ; \boldsymbol{\varsigma})$ into a finite sum of $J$-integrals

$$
J_{v}(x ; \boldsymbol{\varsigma})=\sum_{\varrho \in I^{d}} J_{v}(x ; \boldsymbol{\varsigma} ; \varrho),
$$

with

$$
J_{v}(x ; \boldsymbol{\varsigma} ; \varrho)=J_{v}\left(x ; \boldsymbol{\varsigma} ; h_{\varrho}\right)=\int_{\mathbb{R}_{+}^{d}} h_{\varrho}(\boldsymbol{t}) p_{v}(\boldsymbol{t}) e^{i x \theta(\boldsymbol{t} ; \boldsymbol{s})} d \boldsymbol{t} .
$$

7.3. The Definition of $\mathbb{J}_{v}(x ; \boldsymbol{s})$. Let $a>0$ and assume $\boldsymbol{v} \in \mathbb{S}_{a}$. Let $A \geqslant a+2$ be an integer. For $\varrho \in I^{d}$ denote $L_{ \pm}(\varrho)=\left\{l: \varrho_{l}= \pm\right\}$.

We first treat $J_{v}(x ; \boldsymbol{\xi} ; \varrho)$ in the case when both $L_{+}(\varrho)$ and $L_{-}(\varrho)$ are nonempty. Define $\mathcal{P}_{+, \varrho}=\prod_{l \in L_{-}(\varrho)} \mathcal{P}_{+, l}$. This is well-defined due to commutativity (Lemma 7.3 (3)). By Lemma 7.3 (4) we find that $\mathcal{P}_{+, \varrho}^{2 A}\left(J_{v}(x ; \boldsymbol{\varsigma} ; \varrho)\right)$ is a linear combination of

$$
\begin{aligned}
& \prod_{l \in L_{-}(\varrho)}\left[v_{l}-1\right]_{\alpha_{3 l}} \cdot x^{-2 A\left|L_{-}(\varrho)\right|+\sum_{l \in L_{-}(\varrho)} \alpha_{11}} .
\end{aligned}
$$

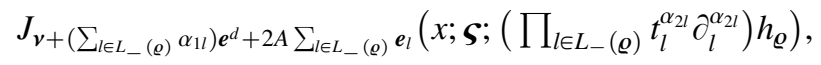

with $\alpha_{1 l}+\alpha_{2 l}+\alpha_{3 l} \leqslant 2 A$ for each $l \in L_{-}(\varrho)$. After this, we choose $l_{+} \in L_{+}(\varrho)$ and apply $\mathcal{P}_{-, l_{+}}^{A+\sum_{l \in L_{-}(\varrho)} \alpha_{1 l}}$ on the $J$-integral in 7.2p. By Lemma 7.3 (4) we obtain a linear combination of

$$
\begin{aligned}
{\left[v_{l_{+}}-1\right]_{\alpha_{3}} \prod_{l \in L_{-}(\varrho)}\left[v_{l}-1\right]_{\alpha_{3 l}} \cdot x^{-A\left(2\left|L_{-}(\varrho)\right|+1\right)+\alpha_{1}} . } \\
J_{\boldsymbol{v}-A \boldsymbol{e}^{d}+2 A \sum_{l \in L_{-}(\varrho)} \boldsymbol{e}_{l}-\alpha_{1} \boldsymbol{e}_{l_{+}}}\left(x ; \boldsymbol{\varsigma} ;\left(t_{l_{+}}^{\alpha_{2}} \partial_{l_{+}}^{\alpha_{2}} \prod_{l \in L_{-}(\varrho)} t_{l}^{\alpha_{2 l}} \partial_{l}^{\alpha_{2 l}}\right) h_{\varrho}\right),
\end{aligned}
$$

with $\alpha_{1}+\alpha_{2}+\alpha_{3} \leqslant \sum_{l \in L_{-}(\varrho)} \alpha_{1 l}+A$. It is easy to verify that the real part of the $l$-th index of the $J$-integral in 7.3 is positive if $l \in L_{-}(\varrho)$ and negative if $l \in L_{+}(\varrho)$. Therefore, the $J$-integral in 7.3 is absolutely convergent according to Lemma 7.3 (1). We define $\mathbb{J}_{v}(x ; \boldsymbol{s}, \varrho)$ to be the total linear combination of all the resulting $J$-integrals.

When $L_{-}(\varrho) \neq \varnothing$ but $L_{+}(\varrho)=\emptyset$, we define $\mathbb{J}_{v}(x ; \boldsymbol{\varsigma} ; \varrho)=\mathcal{P}_{+, \varrho}^{A}\left(J_{v}(x ; \boldsymbol{\varsigma} ; \varrho)\right)$. It is a linear combination of

$$
\begin{aligned}
& \prod_{l \in L_{-}(\varrho)}\left[v_{l}-1\right]_{\alpha_{3 l}} \cdot x^{-A\left|L_{-}(\varrho)\right|+\sum_{l \in L_{-}(\varrho)} \alpha_{11}} .
\end{aligned}
$$

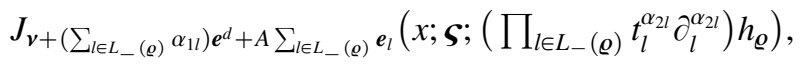

with $\alpha_{1 l}+\alpha_{2 l}+\alpha_{3 l} \leqslant A$. The $J$-integral in (7.4) is absolutely convergent.

When $L_{+}(\varrho) \neq \varnothing$ but $L_{-}(\varrho)=\varnothing$, we choose $l_{+} \in L_{+}(\varrho)$ and define $\mathbb{J}_{\boldsymbol{v}}(x ; \boldsymbol{\varsigma} ; \varrho)=$ $\mathcal{P}_{-, l_{+}}^{A}\left(J_{\boldsymbol{v}}(x ; \boldsymbol{\varsigma} ; \varrho)\right)$. This is a linear combination of

$$
\left[v_{l_{+}}-1\right]_{\alpha_{3}} x^{-A+\alpha_{1}} J_{v-A e^{d}-\alpha_{1} e_{l_{+}}}\left(x ; \boldsymbol{s} ; t_{l_{+}}^{\alpha_{2}} \partial_{l_{+}}^{\alpha_{2}} h_{\varrho}\right),
$$

with $\alpha_{1}+\alpha_{2}+\alpha_{3} \leqslant A$. The $J$-integral in (7.5) is again absolutely convergent.

Finally, when both $L_{-}(\varrho)$ and $L_{+}(\varrho)$ are empty, we put $\mathbb{J}_{\boldsymbol{v}}(x ; \boldsymbol{\varsigma} ; \varrho)=J_{v}(x ; \boldsymbol{\varsigma} ; \varrho)$.

Lemma 7.4. The definition of $\mathbb{J}_{\boldsymbol{v}}(x ; \boldsymbol{\varsigma}, \varrho)$ is independent on $A$ and the choice of $l_{+} \in$ $L_{+}(\varrho)$.

Proof. We shall only consider the case when both $L_{+}(\varrho)$ and $L_{-}(\varrho)$ are nonempty. The other cases are similar and simpler.

Starting from the $\mathbb{J}_{v}(x ; \boldsymbol{\varsigma} ; \varrho)$ defined with $A$, we conduct the following operations in succession for all $l \in L_{-}(\varrho): \mathcal{P}_{+, l}$ twice and then $\mathcal{P}_{-, l_{+}}$once, twice or three times on each 
resulting $J$-integral so that the increment of the $l$-th index is exactly one. In this way, one arrives at the $\mathbb{J}_{v}(x ; \boldsymbol{s} ; \varrho)$ defined with $A+1$. In view of the assumption $A \geqslant a+2$, absolute convergence is maintained at each step due to Lemma 7.3 (1). Moreover, in our settings, the operations $\mathcal{P}_{+, l}$ and $\mathcal{P}_{-, l_{+}}$are actual partial integrations (Lemma $7.3(2)$ ), so the value is preserved in the process. In conclusion, $\mathbb{J}_{v}(x ; \boldsymbol{\varsigma} ; \varrho)$ is independent on $A$.

Suppose $l_{+}, k_{+} \in L_{+}(\varrho)$. Repeating the process described in the last paragraph $A$ times, but with $l_{+}$replaced by $k_{+}$, the $\mathbb{J}_{v}(x ; \boldsymbol{s} ; \varrho)$ defined with $l_{+}$turns into a sum of integrals of an expression symmetric about $l_{+}$and $k_{+}$. Interchanging $l_{+}$and $k_{+}$throughout the arguments above, the $\mathbb{J}_{v}(x ; \boldsymbol{\varsigma} ; \varrho)$ defined with $k_{+}$is transformed into the same sum of integrals. Thus we conclude that $\mathbb{J}_{\boldsymbol{v}}(x ; \boldsymbol{\varsigma} ; \varrho)$ is independent on the choice of $l_{+} \cdot \quad$ Q.E.D.

Putting these together, we define

$$
\mathbb{J}_{v}(x ; \boldsymbol{\varsigma})=\sum_{\varrho \in I^{d}} \mathbb{J}_{v}(x ; \boldsymbol{\varsigma} ; \varrho)
$$

and call $\mathbb{J}_{v}(x ; \boldsymbol{s})$ the rigorous interpretation of $J_{v}(x ; \boldsymbol{s})$. The definition of $\mathbb{J}_{v}(x ; \boldsymbol{\varsigma})$ is clearly independent on the partition of unity $\left\{h_{\varrho}\right\}_{\varrho \in I}$ on $\mathbb{R}_{+}$.

Uniform convergence of the $J$-integrals in 7.3,7.4, 7.5 with respect to $v$ implies that $\mathbb{J}_{\boldsymbol{v}}(x ; \boldsymbol{s})$ is an analytic function of $\boldsymbol{v}$ on $\mathbb{S}_{a}^{d}$ and hence on the whole $\mathbb{C}^{d}$ since $a$ was arbitrary. Moreover, for any nonnegative integer $j$, if one chooses $A \geqslant a+j+2$, differentiating $j$ times under the integral sign for the $J$-integrals in $7.3,7.4,7.5$ is legitimate. Therefore, $\mathbb{J}_{v}(x ; \boldsymbol{s})$ is a smooth function of $x$.

Henceforth, with ambiguity, we shall write $\mathbb{J}_{v}(x ; \boldsymbol{\varsigma})$ and $\mathbb{J}_{v}(x ; \boldsymbol{\varsigma} ; \varrho)$ as $J_{v}(x ; \boldsymbol{\varsigma})$ and $J_{v}(x ; \boldsymbol{\varsigma} ; \varrho)$ respectively.

\section{Equality between $J_{v}(x ; \boldsymbol{\varsigma})$ and $J(x ; \boldsymbol{\varsigma}, \lambda)$}

The goal of this section is to prove that the Bessel function $J(x ; \boldsymbol{\varsigma}, \lambda)$ is indeed equal to the rigorous interpretation of its formal integral representation $J_{v}(x ; \boldsymbol{\varsigma})$.

Proposition 8.1. Suppose that $\lambda \in \mathbb{L}^{d}$ and $v \in \mathbb{C}^{d}$ satisfy $v_{l}=\lambda_{l}-\lambda_{d+1}, l=1, \ldots, d$. Then

$$
J(x ; \boldsymbol{s}, \lambda)=J_{v}(x ; \boldsymbol{\varsigma}) .
$$

To prove this proposition, we first recall the expression of the Hankel transform $\Upsilon=$ $\mathcal{H}_{(\varsigma, \lambda)} v$ as a Fourier type integral transform given in $\$ 5.3 .1$

Suppose that $\mathfrak{R e} \lambda_{1}>\ldots>\mathfrak{R e} \lambda_{d}>\mathfrak{R e} \lambda_{d+1}$. Let $v \in \mathscr{S}\left(\mathbb{R}_{+}\right)$be a Schwartz function on $\mathbb{R}_{+}$. Define

$$
\Upsilon_{d+1}(x)=\mathcal{S}_{\left(\varsigma_{d+1}, \lambda_{d+1}\right)} v(x)=\int_{\mathbb{R}_{+}} v(y) y^{-\lambda_{d+1}} e^{\varsigma_{d+1} i x y} d y, \quad x \in \mathbb{R}_{+},
$$

and, for each $l=1, \ldots, d$, recursively define

$$
\Upsilon_{l}(x)=\mathcal{T}_{\left(\varsigma_{l}, \lambda_{l}-\lambda_{l+1}\right)} \Upsilon_{l+1}(x)=\int_{\mathbb{R}_{+}} \Upsilon_{l+1}(y) y^{\lambda_{l}-\lambda_{l+1}-1} e^{\varsigma_{l} i x y-1} d y, \quad x \in \mathbb{R}_{+} .
$$

It is known from Lemma 5.1 and Lemma 5.5 (4) that $\Upsilon_{d+1} \in \mathscr{S}\left(\overline{\mathbb{R}}_{+}\right) \subset \mathscr{T}_{\text {sis }}\left(\mathbb{R}_{+}\right)$and $\Upsilon_{l} \in \mathscr{T}_{\text {sis }}\left(\mathbb{R}_{+}\right)$for all $l=1, \ldots, d$. Moreover, we have $\Upsilon(x)=x^{-\lambda_{1}} \Upsilon_{1}(x)$. See $\$ 5.3 .1$

The change of variables from $y$ to $x y$ in 8.2 yields

$$
\Upsilon_{l}(x)=\int_{\mathbb{R}_{+}} \Upsilon_{l+1}(x y) x^{\lambda_{l}-\lambda_{l+1}} y^{\lambda_{l}-\lambda_{l+1}-1} e^{\varsigma_{l} i y^{-1}} d y .
$$


Some calculations then show that $\Upsilon_{1}(x)$ is equal to the iterated integral

$$
x^{\nu_{1}} \int_{\mathbb{R}_{+}^{d+1}} v(y) y^{-\lambda_{d+1}}\left(\prod_{l=1}^{d} y_{l}^{\nu_{l}-1}\right) e^{i\left(\varsigma_{d+1} x y y_{1} \ldots y_{d}+\sum_{l=1}^{d} \varsigma \varsigma_{l} y_{l}^{-1}\right)} d y d y_{d} \ldots d y_{1} .
$$

The (actual) partial integration $\mathcal{P}_{l}$ on the integral over $d y_{l}$ is in correspondence with $\mathcal{P}_{+, l}$, whereas the partial integration $\mathcal{P}_{d+1}$ on the integral over $d y$ has the similar effect as $\mathcal{P}_{-, l_{+}}$ of decreasing the powers of all the $y_{l}$ by one. These observations are crucial to our proof of Proposition 8.1 as follows.

Proof of Proposition 8.1. Suppose that $\mathfrak{R e} \lambda_{1}>\ldots>\Re e \lambda_{d}>\mathfrak{R e} \lambda_{d+1}$. We first partition the integral over $d y_{l}$ in 8.3 , for each $l=1, \ldots, d$, into a sum of integrals according to a partition of unity $\left\{h_{\varrho}^{\circ}\right\}_{\varrho \in I}$ of $\mathbb{R}_{+}$. These partitions result in a partition of the integral (8.3) into the sum

with

$$
\Upsilon_{1}(x)=\sum_{\varrho \in I^{d}} \Upsilon_{1}(x ; \varrho)
$$

$$
\Upsilon_{1}(x ; \varrho)=x^{\nu_{1}} \int_{\mathbb{R}_{+}^{d+1}} v(y) y^{-\lambda_{d+1}}\left(\prod_{l=1}^{d} h_{\varrho_{l}}^{\mathrm{o}}\left(y_{l}\right) y_{l}^{\nu_{l}-1}\right) e^{i\left(\varsigma_{d+1} x y y_{1} \ldots y_{d}+\sum_{l=1}^{d} \varsigma y_{l}^{-1}\right)} d y d y_{d} \ldots d y_{1} .
$$

We now conduct the operations in $\$ 7.3$ with $\mathcal{P}_{+, l}$ replaced by $\mathcal{P}_{l}$ and $\mathcal{P}_{-, l_{+}}$by $\mathcal{P}_{d+1}$ to each integral $\Upsilon_{1}(x ; \varrho)$ defined above. While preserving the value, these partial integrations turn the iterated integral $\Upsilon_{1}(x ; \varrho)$ into an absolutely convergent multiple integral. We are then able to move the innermost integral over $d y$ to the outermost place. The integral over $d y_{d} \ldots d y_{1}$ now becomes the inner integral. Making the change of variables $y_{l}=t_{l}(x y)^{-\frac{1}{d+1}}$ to the inner integral over $d y_{d} \ldots d y_{1}$, each partial integration $\mathcal{P}_{l}$ that we did turns into $\mathcal{P}_{+, l}$. By the same arguments in the proof of Lemma 7.4 showing that $J_{v}(x ; \boldsymbol{s})$ is independent on the choice of $l_{+} \in L_{+}(\varrho)$, the operations of $\mathcal{P}_{d+1}$ that we conducted at the beginning may be reversed and substituted by those of $\mathcal{P}_{-, l_{+}}$. It follows that the inner integral over $d y_{d} \ldots d y_{1}$ is equal to $x^{\lambda_{1}} v(y) J_{v}\left((x y)^{\frac{1}{d+1}} ; \boldsymbol{s} ; \varrho\right)$, with $h_{\varrho}(t)=h_{\varrho}^{\mathrm{o}}\left(t(x y)^{-\frac{1}{d+1}}\right)$. Summing over $\varrho \in I^{d}$, we conclude that

$$
\Upsilon(x)=x^{-\lambda_{1}} \Upsilon_{1}(x)=\int_{\mathbb{R}_{+}} v(y) J_{v}\left((x y)^{\frac{1}{d+1}} ; \boldsymbol{\varsigma}\right) d y .
$$

Therefore, in view of 3.5 , we have $J(x ; \boldsymbol{\varsigma}, \lambda)=J_{v}(x ; \boldsymbol{\varsigma})$. This equality holds true universally due to the principle of analytic continuation.

Q.E.D.

In view of Proposition 8.1, we shall subsequently assume that $\lambda \in \mathbb{L}^{d}$ and $v \in \mathbb{C}^{d}$ satisfy the relations $v_{l}=\lambda_{l}-\lambda_{d+1}, l=1, \ldots, d$.

\section{9. $H$-Bessel Functions and $K$-Bessel Functions}

According to Proposition 4.4 $J_{2 \lambda}(x ; \pm, \pm)=J(x ; \pm, \pm, \lambda,-\lambda)$ is a Hankel function, and $J_{2 \lambda}(x ; \pm, \mp)=J(x ; \pm, \mp, \lambda,-\lambda)$ is a $K$-Bessel function. There is a remarkable difference between the behaviours of Hankel functions and the $K$-Bessel function for large argument. Hankel functions oscillate and decay proportionally to $1 / \sqrt{x}$, whereas the $K$ Bessel function exponentially decays. On the other hand, this phenomena also arises in higher rank for the prototypical example shown in Proposition A.1.

In the following, we shall show that such a categorization stands in general for the Bessel functions $J_{v}(x ; \boldsymbol{\varsigma})$ of an arbitrary index $v$. For this, we shall analyze each integral $J_{v}(x ; \boldsymbol{\varsigma} ; \varrho)$ in the rigorous interpretation of $J_{v}(x ; \boldsymbol{\varsigma})$ by the method of stationary phase. 
First of all, the asymptotic behaviour of $J_{v}(x ; \boldsymbol{\varsigma})$ for large argument should rely on the existence of a stationary point of the phase function $\theta(\boldsymbol{t} ; \boldsymbol{\boldsymbol { s }})$ on $\mathbb{R}_{+}^{d}$. We have

$$
\theta^{\prime}(\boldsymbol{t} ; \boldsymbol{s})=\left(\varsigma_{d+1} t_{1} \ldots \hat{l}_{l} \ldots t_{d}-\varsigma_{l} t_{l}^{-2}\right)_{l=1}^{d} .
$$

A stationary point of $\theta(\boldsymbol{t} ; \boldsymbol{\boldsymbol { c }})$ exists in $\mathbb{R}_{+}^{d}$ if and only if $\varsigma_{1}=\ldots=\boldsymbol{S}_{d}=\varsigma_{d+1}$, in which case it is equal to $t_{0}=(1, \ldots, 1)$.

Terminology 9.1. We write $H_{v}^{ \pm}(x)=J_{v}(x ; \pm, \ldots, \pm), H^{ \pm}(x ; \lambda)=J(x ; \pm, \ldots \pm, \lambda)$ and call them $H$-Bessel functions. If two of the signs $\varsigma_{1}, \ldots, \varsigma_{d}, \varsigma_{d+1}$ are different, then $J_{v}(x ; \boldsymbol{\varsigma})$, or $J(x ; \boldsymbol{s}, \lambda)$, is called a $K$-Bessel function.

Preparations. We shall retain the notations in \$7. Moreover, for our purpose we choose a partition of unity $\left\{h_{\varrho}\right\}_{\varrho \in\{-, 0,+\}}$ on $\mathbb{R}_{+}$such that $h_{-}, h_{0}$ and $h_{+}$are functions in $\mathscr{T}\left(\mathbb{R}_{+}\right)$supported on $K_{-}=\left(0, \frac{1}{2}\right], K_{0}=\left[\frac{1}{4}, 4\right]$ and $K_{+}=[2, \infty)$ respectively. Put $K_{\varrho}=\prod_{l=1}^{d} K_{\varrho_{l}}$ and $h_{\varrho}(t)=\prod_{l=1}^{d} h_{\varrho_{l}}\left(t_{l}\right)$ for $\varrho \in\{-, 0,+\}^{d}$. Note that $\boldsymbol{t}_{0}$ is enclosed in the central hypercube $K_{\mathbf{0}}$. According to this partition of unity, $J_{v}(x ; \boldsymbol{s})$ is partitioned into the sum of $3^{d}$ integrals $J_{v}(x ; \boldsymbol{\varsigma} ; \varrho)$. In view of $7.3,7.4,7.5, J_{v}(x ; \boldsymbol{\varsigma} ; \varrho)$ is a $\mathbb{C}\left[x^{-1}\right]$-linear combination of absolutely convergent $J$-integrals of the form

$$
J_{v^{\prime}}(x ; \boldsymbol{s} ; h)=\int_{\mathbb{R}_{+}^{d}} h(\boldsymbol{t}) p_{v^{\prime}}(\boldsymbol{t}) e^{i x \theta(\boldsymbol{t} ; \boldsymbol{s})} d \boldsymbol{t} .
$$

Here $h \in \otimes{ }^{d} \mathscr{T}\left(\mathbb{R}_{+}\right)$is supported in $K_{\varrho}$, and $\boldsymbol{v}^{\prime} \in \boldsymbol{v}+\mathbb{Z}^{d}$ satisfies

$$
\Re \mathrm{e} v_{l}^{\prime}-\mathfrak{R e} v_{l} \geqslant A \text { if } l \in L_{-}(\varrho) \text {, and } \Re \mathrm{e} v_{l}^{\prime}-\mathfrak{R e} v_{l} \leqslant-A \text { if } l \in L_{+}(\varrho) \text {, }
$$

with $A>\max \left\{\left|\Re \mathrm{e} v_{l}\right|\right\}+2$.

9.1. Estimates for $J_{v}(x ; \boldsymbol{\varsigma} ; \varrho)$ with $\varrho \neq \mathbf{0}$. Let

$$
\Theta(\boldsymbol{t} ; \boldsymbol{\zeta})=\sum_{l=1}^{d}\left(t_{l} \partial_{l} \theta(\boldsymbol{t} ; \boldsymbol{\zeta})\right)^{2}=\sum_{l=1}^{d}\left(\varsigma_{d+1} t_{1} \ldots t_{d}-\boldsymbol{\varsigma}_{l} t_{l}^{-1}\right)^{2} .
$$

Lemma 9.2. Let $\varrho \neq \mathbf{0}$. We have for all $\boldsymbol{t} \in K_{\varrho}$

$$
\Theta(t ; \boldsymbol{s}) \geqslant \frac{1}{16}
$$

PRoof. Instead, we shall prove

$$
\max \left\{\left|\varsigma_{d+1} t_{1} \ldots t_{d}-\varsigma_{l} t_{l}^{-1}\right|: t \in \mathbb{R}_{+}^{d} \backslash K_{\mathbf{0}} \text { and } l=1, \ldots, d\right\} \geqslant \frac{1}{4} .
$$

Firstly, if $t_{1} \ldots t_{d}<\frac{3}{4}$, then there exists $t_{l}<1$ and hence $\left|S_{d+1} t_{1} \ldots t_{d}-S_{l} t_{l}^{-1}\right|>1-\frac{3}{4}=\frac{1}{4}$. Similarly, if $t_{1} \ldots t_{d}>\frac{7}{4}$, then there exists $t_{l}>1$ and hence $\left|S_{d+1} t_{1} \ldots t_{d}-S_{l} t_{l}^{-1}\right|>\frac{7}{4}-1>\frac{1}{4}$. Finally, suppose that $t_{1} \ldots t_{d} \in\left[\frac{3}{4}, \frac{7}{4}\right]$, then for our choice of $t$ there exists $t_{l} \notin\left(\frac{1}{2}, 2\right)$, and therefore we still have $\left|S_{d+1} t_{1} \ldots t_{d}-\varsigma_{l} t_{l}^{-1}\right| \geqslant \frac{1}{4}$.

Q.E.D.

Using 9.3 , we rewrite the $J$-integral $J_{\boldsymbol{v}^{\prime}}(x ; \boldsymbol{\varsigma} ; h)$ in 9.1 as below,

$$
\sum_{l=1}^{d} \int_{\mathbb{R}_{+}^{d}} h(\boldsymbol{t})\left(\varsigma_{d+1} p_{\boldsymbol{v}^{\prime}+\boldsymbol{e}^{d}+\boldsymbol{e}_{l}}(\boldsymbol{t})-\boldsymbol{\varsigma}_{l} p_{\boldsymbol{v}^{\prime}}(\boldsymbol{t})\right) \Theta(\boldsymbol{t} ; \boldsymbol{\zeta})^{-1} \cdot \partial_{l} \theta(\boldsymbol{t} ; \boldsymbol{\xi}) e^{i x \theta(\boldsymbol{t} ; \boldsymbol{\xi})} d \boldsymbol{t}
$$

We now make use of the third kind of partial integrations arising from

$$
\partial\left(e^{i x \theta(\boldsymbol{t} ; \boldsymbol{s})}\right)=i x \cdot \partial_{l} \theta(\boldsymbol{t} ; \boldsymbol{s}) e^{i x \theta(\boldsymbol{t} ; \boldsymbol{s})} \partial t_{l} .
$$


For the $l$-th integral in 9.4 , we apply the corresponding partial integration of the third kind. In this way, (9.4) turns into

$$
\begin{aligned}
& -(i x)^{-1} \sum_{l=1}^{d} \int_{\mathbb{R}_{+}^{d}} t_{l} \partial_{l} h\left(\varsigma_{d+1} p_{\boldsymbol{v}^{\prime}+\boldsymbol{e}^{d}}-\varsigma_{l} p_{\boldsymbol{v}^{\prime}-\boldsymbol{e}_{l}}\right) \Theta^{-1} e^{i x \theta} d \boldsymbol{t} \\
& -(i x)^{-1} \sum_{l=1}^{d} \int_{\mathbb{R}_{+}^{d}} h\left(\varsigma_{d+1}\left(v_{l}^{\prime}+1\right) p_{\boldsymbol{v}^{\prime}+\boldsymbol{e}^{d}}-\varsigma_{l}\left(v_{l}^{\prime}-1\right) p_{\boldsymbol{v}^{\prime}-\boldsymbol{e}_{l}}\right) \Theta^{-1} e^{i x \theta} d \boldsymbol{t} \\
& +\boldsymbol{\varsigma}_{d+1} 2 d^{2}(i x)^{-1} \int_{\mathbb{R}_{+}^{d}} h p_{\boldsymbol{v}^{\prime}+3 \boldsymbol{e}^{d}} \Theta^{-2} e^{i x \theta} d \boldsymbol{t} \\
& +2(i x)^{-1} \sum_{l=1}^{d} \int_{\mathbb{R}_{+}^{d}} h\left(\varsigma_{l}(1-2 d) p_{\boldsymbol{v}^{\prime}+2 \boldsymbol{e}^{d}-\boldsymbol{e}_{l}}-\varsigma_{d+1} p_{\boldsymbol{v}^{\prime}+\boldsymbol{e}^{d}-2 \boldsymbol{e}_{l}}+\varsigma_{l} p_{\boldsymbol{v}^{\prime}-3 \boldsymbol{e}_{l}}\right) \Theta^{-2} e^{i x \theta} d \boldsymbol{t} \\
& +4(i x)^{-1} \sum_{1 \leqslant l<k \leqslant d} \varsigma_{d+1} \varsigma_{l} \varsigma_{k} \int_{\mathbb{R}_{+}^{d}} h p_{\boldsymbol{v}^{\prime}+\boldsymbol{e}^{d}-\boldsymbol{e}_{l}-\boldsymbol{e}_{k}} \Theta^{-2} e^{i x \theta} d \boldsymbol{t},
\end{aligned}
$$

where $\Theta$ and $\theta$ are the shorthand notations for $\Theta(\boldsymbol{t} ; \boldsymbol{s})$ and $\theta(\boldsymbol{t} ; \boldsymbol{s})$. Since the shifts of indices do not exceed 3 , it follows from the condition 9.2 , combined with Lemma 9.2. that all the integrals above absolutely converge provided $A>\max \left\{\left|\Re e v_{l}\right|\right\}+3$.

Repeating the above manipulations, we obtain the following lemma by a straightforward inductive argument.

Lemma 9.3. Let $B$ be a nonnegative integer, and choose $A=\left\lfloor\max \left\{\left|\Re e v_{l}\right|\right\}\right\rfloor+3 B+3$. Then $J_{v^{\prime}}(x ; \boldsymbol{s} ; h)$ is equal to a linear combination of $\left(\frac{1}{2}\left(d^{2}-d\right)+7 d+1\right)^{B}$ many absolutely convergent integrals of the following form

$$
(i x)^{-B} P\left(\boldsymbol{v}^{\prime}\right) \int_{\mathbb{R}_{+}^{d}} \boldsymbol{t}^{\alpha} \partial^{\alpha} h(\boldsymbol{t}) p_{\boldsymbol{v}^{\prime \prime}}(\boldsymbol{t}) \Theta(\boldsymbol{t} ; \boldsymbol{s})^{-B-B_{2}} e^{i x \theta(\boldsymbol{t} ; \boldsymbol{s})} d \boldsymbol{t},
$$

where $|\boldsymbol{\alpha}|+B_{1}+B_{2}=B\left(\boldsymbol{\alpha} \in \mathbb{N}^{d}\right)$, $P$ is a polynomial of degree $B_{1}$ and integer coefficients of size $O_{B, d}(1)$, and $\boldsymbol{v}^{\prime \prime} \in \boldsymbol{v}^{\prime}+\mathbb{Z}^{d}$ satisfies $\left|v_{l}^{\prime \prime}-v_{l}^{\prime}\right| \leqslant B+2 B_{2}$ for all $l=1, \ldots, d$. Recall that in the multi-index notation $|\boldsymbol{\alpha}|=\sum_{l=1}^{d} \alpha_{l}, \boldsymbol{t}^{\alpha}=\prod_{l=1}^{d} t_{l}^{\alpha_{l}}$ and $\partial^{\alpha}=\prod_{l=1}^{d} \partial_{l}^{\alpha_{l}}$.

Define $\mathfrak{c}=\max \left\{\left|v_{l}\right|\right\}+1$ and $\mathfrak{r}=\max \left\{\left|\mathfrak{R e} v_{l}\right|\right\}$. Suppose that $x \geqslant \mathfrak{c}$. Applying Lemma 9.3 and 9.2 to the $J$-integrals in 7.3,7.4,7.5, , one obtains the estimate

$$
J_{v}(x ; \boldsymbol{\varsigma} ; \varrho) \ll_{\mathrm{r}, M, d}\left(\frac{\mathfrak{c}}{x}\right)^{M}
$$

for any given nonnegative integer $M$. Slight modifications of the above arguments yield a similar estimate for the derivative

$$
J_{v}^{(j)}(x ; \boldsymbol{s} ; \varrho) \ll_{\mathrm{r}, M, j, d}\left(\frac{\mathfrak{c}}{x}\right)^{M} .
$$

Remark 9.4. Our proof of (9.5) is similar to that of [Hör Theorem 7.7.1]. Indeed, $\Theta(t ; \boldsymbol{s})$ plays the same role as $\left|f^{\prime}\right|^{2}+\mathfrak{I} m f$ in the proof of [Hör , Theorem 7.7.1], where $f$ is the phase function there. The non-compactness of $K_{\varrho}$ however prohibits the application of [Hör Theorem 7.7.1] to the $J$-integral in 9.1] in our case.

9.2. Rapid Decay of $K$-Bessel Functions. Suppose that there exists $l$ such that $\varsigma_{l} \neq$ $\varsigma_{d+1}$. Then for any $t \in K_{\mathbf{0}}$

$$
\left|\varsigma_{d+1} t_{1} \ldots t_{d}-\varsigma_{l} t_{l}^{-1}\right|>t_{l}^{-1} \geqslant \frac{1}{4} .
$$


Similar to the arguments in $\$ 9.1$, repeating the $l$-th partial integration of the third kind yields the same bound 9.5 in the case $\varrho=\mathbf{0}$.

Remark 9.5. For this, we may also directly apply [Hör, Theorem 7.7.1].

THEOREM 9.6. Let $\mathrm{c}=\max \left\{\left|v_{l}\right|\right\}+1$ and $\mathrm{r}=\max \left\{\left|\Re \mathrm{Re} v_{l}\right|\right\}$. Let $j$ and $M$ be nonnegative integers. Suppose that one of the signs $\varsigma_{1}, \ldots, \varsigma_{d}$ is different from $\varsigma_{d+1}$. Then

$$
J_{v}^{(j)}(x ; \boldsymbol{s}) \ll_{\mathrm{r}, M, j, d}\left(\frac{\mathfrak{c}}{x}\right)^{M}
$$

for any $x \geqslant c$. In particular, $J_{v}(x ; \boldsymbol{\varsigma})$ is a Schwartz function at infinity, namely, all the derivatives $J_{v}^{(j)}(x ; \boldsymbol{\varsigma})$ rapidly decay at infinity.

9.3. Asymptotic Expansions of $H$-Bessel Functions. In the following, we shall adopt the convention $( \pm i)^{a}=e^{ \pm \frac{1}{2} i \pi a}, a \in \mathbb{C}$.

We first introduce the function $W_{v}^{ \pm}(x)$, which is closely related to the Whittaker function of imaginary argument if $d=1$ (see [WW] $\$ 17.5,17.6]$ ), defined by

$$
W_{\nu}^{ \pm}(x)=(d+1)^{\frac{1}{2}}( \pm 2 \pi i)^{-\frac{d}{2}} e^{\mp i(d+1) x} H_{\nu}^{ \pm}(x) .
$$

Write $H_{v}^{ \pm}(x ; \varrho)=J_{v}(x ; \pm, \ldots, \pm ; \varrho)$ and define

$$
W_{v}^{ \pm}(x ; \varrho)=(d+1)^{\frac{1}{2}}( \pm 2 \pi i)^{-\frac{d}{2}} e^{\mp i(d+1) x} H_{\nu}^{ \pm}(x ; \varrho) .
$$

For $\varrho \neq \mathbf{0}$, the bound 9.5 for $H_{v}^{ \pm}(x ; \varrho)$ is also valid for $W_{v}^{ \pm}(x ; \varrho)$. Therefore, we are left with analyzing $W_{v}^{ \pm}(x ; \mathbf{0})$. We have

$$
\begin{aligned}
W_{v}^{ \pm,(j)}(x ; \mathbf{0})= & (d+1)^{\frac{1}{2}}( \pm 2 \pi i)^{-\frac{d}{2}}( \pm i)^{j} \\
& \int_{K_{\mathbf{0}}}(\theta(\boldsymbol{t})-d-1)^{j} h_{\mathbf{0}}(\boldsymbol{t}) p_{\boldsymbol{v}}(\boldsymbol{t}) e^{ \pm i x(\theta(\boldsymbol{t})-d-1)} d \boldsymbol{t},
\end{aligned}
$$

with

$$
\theta(\boldsymbol{t})=\theta(\boldsymbol{t} ;+, \ldots,+)=t_{1} \ldots t_{d}+\sum_{l=1}^{d} t_{l}^{-1} .
$$

Proposition 9.7. [Hör, Theorem 7.7.5]. Let $K \subset \mathbb{R}^{d}$ be a compact set, $X$ an open neighbourhood of $K$ and $M$ a nonnegative integer. If $u(t) \in C_{0}^{2 M}(K), f(\boldsymbol{t}) \in C^{3 M+1}(X)$ and $\mathfrak{I m} f \geqslant 0$ in $X$, Im $f\left(\boldsymbol{t}_{0}\right)=0, f^{\prime}\left(\boldsymbol{t}_{0}\right)=0$, det $f^{\prime \prime}\left(\boldsymbol{t}_{0}\right) \neq 0$ and $f^{\prime} \neq 0$ in $K \backslash\left\{\boldsymbol{t}_{0}\right\}$, then for $x>0$

$\left|\int_{K} u(\boldsymbol{t}) e^{i x f(\boldsymbol{t})} d \boldsymbol{t}-e^{i x f\left(\boldsymbol{t}_{0}\right)}\left((2 \pi i)^{-d} \operatorname{det} f^{\prime \prime}\left(\boldsymbol{t}_{0}\right)\right)^{-\frac{1}{2}} \sum_{m=0}^{M-1} x^{-m-\frac{d}{2}} \mathcal{L}_{m} u\right| \ll x^{-M} \sum_{|\alpha| \leqslant 2 M} \sup \left|D^{\alpha} u\right|$.

Here the implied constant depends only on $M, f, K$ and $d$. With

$$
g(\boldsymbol{t})=f(\boldsymbol{t})-f\left(\boldsymbol{t}_{0}\right)-\frac{1}{2}\left\langle f^{\prime \prime}\left(\boldsymbol{t}_{0}\right)\left(\boldsymbol{t}-\boldsymbol{t}_{0}\right), \boldsymbol{t}-\boldsymbol{t}_{0}\right\rangle
$$

which vanishes of third order at $\boldsymbol{t}_{0}$, we have

$$
\left.\mathcal{L}_{m} u=i^{-m} \sum_{r=0}^{2 m} \frac{1}{2^{m+r}(m+r) ! r !}\left\langle f^{\prime \prime}\left(\boldsymbol{t}_{0}\right)^{-1} D, D\right\rangle^{m+r}\left(g^{r} u\right)\left(\boldsymbol{t}_{0}\right)\right]^{\mathrm{II}}
$$

This is a differential operator of order $2 m$ acting on $u$ at $\boldsymbol{t}_{0}$. The coefficients are rational homogeneous functions of degree $-m$ in $f^{\prime \prime}\left(\boldsymbol{t}_{0}\right), \ldots, f^{(2 m+2)}\left(\boldsymbol{t}_{0}\right)$ with denominator $\left(\operatorname{det} f^{\prime \prime}\left(\boldsymbol{t}_{0}\right)\right)^{3 m}$. In every term the total number of derivatives of $u$ and of $f^{\prime \prime}$ is at most $2 m$.

\footnotetext{
${ }^{\mathrm{II}}$ According to Hörmander, $D=-i\left(\partial_{1}, \ldots, \partial_{d}\right)$.
} 
We now apply Proposition 9.7 to the integral in 9.6. For this, we let

$$
\begin{aligned}
& K=K_{\mathbf{0}}=\left[\frac{1}{4}, 4\right]^{d}, \quad X=\left(\frac{1}{5}, 5\right)^{d}, \\
& f(\boldsymbol{t})= \pm(\theta(\boldsymbol{t})-d-1), \quad f^{\prime}(\boldsymbol{t})= \pm\left(t_{1} \ldots \hat{t}_{l} \ldots t_{d}-t_{l}^{-2}\right)_{l=1}^{d}, \quad \boldsymbol{t}_{0}=(1, \ldots, 1), \\
& f^{\prime \prime}\left(\boldsymbol{t}_{0}\right)= \pm\left(\begin{array}{cccc}
2 & 1 & \cdots & 1 \\
1 & 2 & \cdots & 1 \\
\vdots & \vdots & \ddots & \vdots \\
1 & 1 & \cdots & 2
\end{array}\right), \quad \operatorname{det} f^{\prime \prime}\left(\boldsymbol{t}_{0}\right)=( \pm)^{d}(d+1), \quad g(\boldsymbol{t})= \pm G(\boldsymbol{t}), \\
& f^{\prime \prime}\left(\boldsymbol{t}_{0}\right)^{-1}= \pm \frac{1}{d+1}\left(\begin{array}{cccc}
d & -1 & \cdots & -1 \\
-1 & d & \cdots & -1 \\
\vdots & \vdots & \ddots & \vdots \\
-1 & -1 & \cdots & d
\end{array}\right), \\
& u(\boldsymbol{t})=(d+1)^{\frac{1}{2}}( \pm 2 \pi i)^{-\frac{d}{2}}( \pm i)^{j}(\theta(\boldsymbol{t})-d-1)^{j} p_{\boldsymbol{v}}(\boldsymbol{t}) h_{\mathbf{0}}(\boldsymbol{t}),
\end{aligned}
$$

with

$$
G(\boldsymbol{t})=t_{1} \ldots t_{d}+\sum_{l=1}^{d}\left(-t_{l}^{2}+(d+1) t_{l}+t_{l}^{-1}\right)-\sum_{1 \leqslant l<k \leqslant d} t_{l} t_{k}-\frac{(d+1)(d+2)}{2} .
$$

Proposition 9.7 yields the following asymptotic expansion of $W_{v}^{ \pm,(j)}(x ; \mathbf{0})$,

$$
W_{v}^{ \pm,(j)}(x ; \mathbf{0})=\sum_{m=0}^{M-1}( \pm i)^{j-m} B_{m, j}(\boldsymbol{v}) x^{-m-\frac{d}{2}}+O_{\mathfrak{r}, M, j, d}\left(\mathfrak{c}^{2 M} x^{-M}\right), \quad x>0,
$$

with

$$
B_{m, j}(\boldsymbol{v})=\sum_{r=0}^{2 m} \frac{(-)^{m+r} \mathcal{L}^{m+r}\left(G^{r}(\theta-d-1)^{j} p_{\boldsymbol{v}}\right)\left(\boldsymbol{t}_{0}\right)}{(2(d+1))^{m+r}(m+r) ! r !},
$$

in which $\mathcal{L}$ is the second-order differential operator given by

$$
\mathcal{L}=d \sum_{l=1}^{d} \partial_{l}^{2}-2 \sum_{1 \leqslant l<k \leqslant d} \partial_{l} \partial_{k}
$$

Lemma 9.8. We have $B_{m, j}(v)=0$ if $m<j$. Otherwise, $B_{m, j}(v) \in \mathbb{Q}[v]$ is a symmetric polynomial of degree $2 m-2 j$. In particular, $B_{m, j}(v) \ll_{m, j, d} c^{2 m-2 j}$ for $m \geqslant j$.

Proof. The symmetry of $B_{m, j}(v)$ is clear from definition. Since $\theta-d-1$ vanishes of second order at $t_{0}, 2 j$ many differentiations are required to remove the zero of $(\theta-d-1)^{j}$ at $\boldsymbol{t}_{0}$. From this, along with the descriptions of the differential operator $\mathcal{L}_{m}$ in Proposition 9.7. one proves the lemma.

Q.E.D.

Furthermore, in view of the bound $(9.5)$, the total contribution to $W_{v}^{ \pm,(j)}(x)$ from all the $W_{v}^{ \pm,(j)}(x ; \varrho)$ with $\varrho \neq \mathbf{0}$ is of size $O_{\mathfrak{r}, M, j, d}\left(\mathfrak{c}^{M} x^{-M}\right)$ and hence may be absorbed into the error term in the asymptotic expansion of $W_{v}^{ \pm,(j)}(x ; \mathbf{0})$.

In conclusion, the following proposition is established.

Proposition 9.9. Let $M, j$ be nonnegative integers such that $M \geqslant j$. Then for $x \geqslant \mathfrak{c}$

$$
W_{v}^{ \pm,(j)}(x)=\sum_{m=j}^{M-1}( \pm i)^{j-m} B_{m, j}(\boldsymbol{v}) x^{-m-\frac{d}{2}}+O_{\mathrm{r}, M, j, d}\left(\mathrm{c}^{2 M} x^{-M}\right) .
$$


Corollary 9.10. Let $N$, j be nonnegative integers such that $N \geqslant j$, and let $\epsilon>0$.

(1). We have $W_{v}^{ \pm,(j)}(x) \ll_{\mathrm{r}, j, d} \mathrm{c}^{2 j} x^{-j}$ for $x \geqslant \mathrm{c}$.

(2). If $x \geqslant \mathfrak{c}^{2+\epsilon}$, then

$$
W_{v}^{ \pm,(j)}(x)=\sum_{m=j}^{N-1}( \pm i)^{j-m} B_{m, j}(\boldsymbol{v}) x^{-m-\frac{d}{2}}+O_{\mathfrak{r}, N, j, \epsilon, d}\left(\mathfrak{c}^{2 N} x^{-N-\frac{d}{2}}\right) .
$$

Proof. On letting $M=j$, Proposition 9.9 implies (1). On choosing $M$ sufficiently large so that $(2+\epsilon)\left(M-N+\frac{d}{2}\right) \geqslant 2(M-N)$, Proposition 9.9 and Lemma 9.8 yield

$$
\begin{aligned}
& W_{v}^{ \pm,(j)}(x)-\sum_{m=j}^{N-1}( \pm i)^{j-m} B_{m, j}(v) x^{-m-\frac{d}{2}} \\
= & \sum_{m=N}^{M-1}( \pm i)^{j-m} B_{m, j}(v) x^{-m-\frac{d}{2}}+O_{\mathfrak{r}, j, M, d}\left(\mathfrak{c}^{2 M} x^{-M}\right)=O_{\mathrm{r}, j, N, \epsilon, d}\left(\mathfrak{c}^{2 N} x^{-N-\frac{d}{2}}\right) .
\end{aligned}
$$

Q.E.D.

Finally, the asymptotic expansion of $H^{ \pm}(x ; \lambda)\left(=H_{v}^{ \pm}(x)\right)$ is formulated as below.

Theorem 9.11. Let $\mathfrak{C}=\max \left\{\left|\lambda_{l}\right|\right\}+1$ and $\Re=\max \left\{\left|\Re \mathrm{e} \lambda_{l}\right|\right\}$. Let $M$ be a nonnegative integer.

(1). Define $W^{ \pm}(x ; \lambda)=\sqrt{n}( \pm 2 \pi i)^{-\frac{n-1}{2}} e^{\mp i n x} H^{ \pm}(x ; \lambda)$. Let $M \geqslant j \geqslant 0$. Then

$$
W^{ \pm,(j)}(x ; \lambda)=\sum_{m=j}^{M-1}( \pm i)^{j-m} B_{m, j}(\lambda) x^{-m-\frac{n-1}{2}}+O_{\Re, M, j, n}\left(\mathfrak{C}^{2 M} x^{-M}\right)
$$

for all $x \geqslant \mathfrak{C}$. Here $B_{m, j}(\lambda) \in \mathbb{Q}[\lambda]$ is a symmetric polynomial in $\lambda$ of degree $2 m$, with $B_{0,0}(\lambda)=1$. The coefficients of $B_{m, j}(\lambda)$ depends only on $m$, $j$ and $d$.

(2). Let $B_{m}(\lambda)=B_{m, 0}(\lambda)$. Then for $x \geqslant \mathfrak{C}$

$H^{ \pm}(x ; \lambda)=n^{-\frac{1}{2}}( \pm 2 \pi i)^{\frac{n-1}{2}} e^{ \pm i n x} x^{-\frac{n-1}{2}}\left(\sum_{m=0}^{M-1}( \pm i)^{-m} B_{m}(\lambda) x^{-m}+O_{\Re, M, d}\left(\mathfrak{C}^{2 M} x^{-M+\frac{n-1}{2}}\right)\right)$.

Proof. This theorem is a direct consequence of Proposition 9.9 and Lemma 9.8 It is only left to verify the symmetry of $B_{m, j}(\lambda)=B_{m, j}(v)$ with respect to $\lambda$. Indeed, in view of the definition of $H^{ \pm}(x ; \lambda)$ by $(1.2)$ and $(3.6), H^{ \pm}(x ; \lambda)$ is symmetric with respect to $\lambda$, so $B_{m, j}(\lambda)$ must be represented by a symmetric polynomial in $\lambda$ modulo $\sum_{l=1}^{d+1} \lambda_{l}$. Q.E.D.

Corollary 9.12. Let $M$ be a nonnegative integer, and let $\epsilon>0$. Then for $x \geqslant \mathfrak{C}^{2+\epsilon}$ $H^{ \pm}(x ; \lambda)=n^{-\frac{1}{2}}( \pm 2 \pi i)^{\frac{n-1}{2}} e^{ \pm i n x} x^{-\frac{n-1}{2}}\left(\sum_{m=0}^{M-1}( \pm i)^{-m} B_{m}(\lambda) x^{-m}+O_{\Re, M, \epsilon, n}\left(\mathfrak{C}^{2 M} x^{-M}\right)\right)$.

\subsection{Concluding Remarks.}

9.4.1. On the Analytic Continuations of H-Bessel Functions. Our observation is that the phase function $\theta$ defined by 9.7 is always positive on $\mathbb{R}_{+}^{d}$. It follows that if one replaces $x$ by $z=x e^{i \omega}$, with $x>0$ and $0 \leqslant \pm \omega \leqslant \pi$, then the various $J$-integrals in the rigorous interpretation of $H_{v}^{ \pm}(z)$ remain absolutely convergent, uniformly with respect to $z$, since $\left|e^{ \pm i z \theta(t)}\right|=e^{\mp x \sin \omega \theta(t)} \leqslant 1$. Therefore, the resulting integral $H_{v}^{ \pm}(z)$ gives rise to an analytic continuation of $H_{\nu}^{ \pm}(x)$ onto the half-plane $\mathbb{H}^{ \pm}=\{z \in \mathbb{C} \backslash\{0\}: 0 \leqslant \pm \arg z \leqslant \pi\}$. In view of Proposition 8.1 , one may define $H^{ \pm}(z ; \lambda)=H_{v}^{ \pm}(z)$ and regard it as the analytic continuation of $H^{ \pm}(x ; \lambda)$ from $\mathbb{R}_{+}$onto $\mathbb{H}^{ \pm}$. Furthermore, with slight modifications of 
the arguments above, where the phase function $f$ is now chosen to be $\pm e^{i \omega}(\theta-d-1)$ in the application of Proposition 9.7, the domain of validity for the asymptotic expansions in Theorem 9.11 may be extended from $\mathbb{R}_{+}$onto $\mathbb{H}^{ \pm}$. For example, we have

$$
\begin{aligned}
H^{ \pm}(z ; \lambda)=n^{-\frac{1}{2}} & ( \pm 2 \pi i)^{\frac{n-1}{2}} e^{ \pm i n z} z^{-\frac{n-1}{2}} \\
& \left(\sum_{m=0}^{M-1}( \pm i)^{-m} B_{m}(\lambda) z^{-m}+O_{\Re, M, n}\left(\mathfrak{C}^{2 M}|z|^{-M+\frac{n-1}{2}}\right)\right),
\end{aligned}
$$

for all $z \in \mathbb{H}^{ \pm}$such that $|z| \geqslant \mathfrak{C}$.

Obviously, the above method of obtaining the analytic continuation of $H_{v}^{ \pm}$does not apply to $K$-Bessel functions.

9.4.2. On the Implied Constants of Estimates. All the implied constants that occur in this section are of exponential dependence on the real parts of the indices. If one considers the $d$-th symmetric lift of a holomorphic Hecke cusp form of weight $k$, the estimates are particularly awful in the $k$ aspect.

In $\$ 10$ and $\$ 11$, we shall further explore the theory of Bessel functions from the perspective of differential equations. Consequently, if the argument is sufficiently large, then all the estimates in this section can be improved so that the dependence on the index can be completely eliminated.

9.4.3. On the Coefficients in the Asymptotics. One feature of the method of stationary phase is the explicit formula of the coefficients in the asymptotic expansion in terms of certain partial differential operators. In the present case of $H^{ \pm}(x ; \lambda)=H_{v}^{ \pm}(x), 9.9$ ) provides an explicit formula of $B_{m}(\lambda)=B_{m, 0}(v)$. To compute $\mathcal{L}^{m+r}\left(G^{r} p_{v}\right)\left(\boldsymbol{t}_{0}\right)$ appearing in 9.9), we observe that the function $G$ defined in 9.8) does not only vanish of third order at $\boldsymbol{t}_{0}$. Actually, $\partial^{\alpha} G\left(\boldsymbol{t}_{0}\right)$ vanishes except for $\alpha=(0, \ldots, 0, \alpha, 0 \ldots, 0)$, with $\alpha \geqslant 3$. In the exceptional case we have $\partial^{\alpha} G\left(t_{0}\right)=(-)^{\alpha} \alpha$ !. However, the resulting expression is considerably complicated.

When $d=1$, by the definition 9.10, we have $\mathcal{L}=(d / d t)^{2}$. For $2 m \geqslant r \geqslant 1$,

$$
\begin{aligned}
& (d / d t)^{2 m+2 r}\left(G^{r} p_{v}\right)(1) \\
= & (2 m+2 r) ! \sum_{\alpha=0}^{2 m-r}\left|\left\{\left(\alpha_{1}, \ldots, \alpha_{r}\right): \sum_{q=1}^{r} \alpha_{q}=2 m+2 r-\alpha, \alpha_{q} \geqslant 3\right\}\right| \frac{(-)^{\alpha}[v-1]_{\alpha}}{\alpha !} \\
= & (2 m+2 r) ! \sum_{\alpha=0}^{2 m-r}\left(\begin{array}{c}
2 m-\alpha-1 \\
r-1
\end{array}\right) \frac{(1-v)_{\alpha}}{\alpha !} .
\end{aligned}
$$

Therefore 9.9) yields

$$
B_{m, 0}(v)=\left(-\frac{1}{4}\right)^{m}\left(\frac{(1-v)_{2 m}}{m !}+\sum_{r=1}^{2 m} \frac{(-)^{r}(2 m+2 r) !}{4^{r}(m+r) ! r !} \sum_{\alpha=0}^{2 m-r}\left(\begin{array}{c}
2 m-\alpha-1 \\
r-1
\end{array}\right) \frac{(1-v)_{\alpha}}{\alpha !}\right) .
$$

However, this expression of $B_{m, 0}(v)$ is more involved than what is known in the literature. Indeed, we have the asymptotic expansions of $H_{v}^{(1)}$ and $H_{v}^{(2)}$ ([Wat $\left.7.2(1,2)\right]$ )

$$
H_{v}^{(1,2)}(x) \sim\left(\frac{2}{\pi x}\right)^{\frac{1}{2}} e^{ \pm i\left(x-\frac{1}{2} v \pi-\frac{1}{4} \pi\right)}\left(\sum_{m=0}^{\infty} \frac{( \pm)^{m}\left(\frac{1}{2}-v\right)_{m}\left(\frac{1}{2}+v\right)_{m}}{m !(2 i x)^{m}}\right),
$$


which are deducible from Hankel's integral representations ([Wat $6.12(3,4)]$ ). In view of Proposition 4.4 and Theorem 9.11, we have

$$
B_{m, 0}(v)=\frac{\left(\frac{1}{2}-v\right)_{m}\left(\frac{1}{2}+v\right)_{m}}{4^{m} m !} .
$$

Therefore, we deduce the following combinatorial identity

$$
\begin{aligned}
\frac{(-)^{m}\left(\frac{1}{2}-v\right)_{m}\left(\frac{1}{2}+v\right)_{m}}{m !} & = \\
\frac{(1-v)_{2 m}}{m !} & +\sum_{r=1}^{2 m} \frac{(-)^{r}(2 m+2 r) !}{4 r(m+r) ! r !} \sum_{\alpha=0}^{2 m-r}\left(\begin{array}{c}
2 m-\alpha-1 \\
r-1
\end{array}\right) \frac{(1-v)_{\alpha}}{\alpha !} .
\end{aligned}
$$

It seems however hard to find an elementary proof of this identity

\section{Recurrence Formulae and the Differential Equations for Bessel Functions}

Making use of certain recurrence formulae for $J_{v}(x ; \boldsymbol{\varsigma})$, we shall derive the differential equation satisfied by $J(x ; \boldsymbol{\varsigma}, \lambda)$.

10.1. Recurrence Formulae. Applying the formal partial integrations of either the first or the second kind and the differentiation under the integral sign on the formal integral expression of $J_{\boldsymbol{v}}(x ; \boldsymbol{s})$ in 7.1 , one obtains the recurrence formulae

$$
v_{l}(i x)^{-1} J_{\boldsymbol{v}}(x ; \boldsymbol{\varsigma})=\varsigma_{l} J_{\boldsymbol{v}-\boldsymbol{e}_{l}}(x ; \boldsymbol{\varsigma})-\varsigma_{d+1} J_{\boldsymbol{v}+\boldsymbol{e}^{d}}(x ; \boldsymbol{\varsigma})
$$

for $l=1, \ldots, d$, and

$$
J_{\boldsymbol{v}}^{\prime}(x ; \boldsymbol{\varsigma})=\varsigma_{d+1} i J_{\boldsymbol{v}+\boldsymbol{e}^{d}}(x ; \boldsymbol{\varsigma})+i \sum_{l=1}^{d} \varsigma_{l} J_{\boldsymbol{v}-\boldsymbol{e}_{l}}(x ; \boldsymbol{\varsigma}) .
$$

It is easy to verify 10.1 and 10.2$)$ using the rigorous interpretation of $J_{v}(x ; \boldsymbol{\varsigma})$ established in $\$ 7.3$. Alternatively, one may prove these by the Mellin-Barnes integral representation (3.6) of $J(x ; \boldsymbol{s}, \lambda)$. Moreover, using (10.1), one may reformulate 10.2 ) as below,

$$
J_{v}^{\prime}(x ; \boldsymbol{\varsigma})=\varsigma_{d+1} i(d+1) J_{v+e^{d}}(x ; \boldsymbol{\varsigma})+\frac{\sum_{l=1}^{d} v_{l}}{x} J_{v}(x ; \boldsymbol{\varsigma}) .
$$

\subsection{The Differential Equations.}

Lemma 10.1. Define $\boldsymbol{e}^{l}=(\underbrace{1, \ldots, 1}_{l}, 0 \ldots, 0), l=1, \ldots, d$, and denote $\boldsymbol{e}^{0}=\boldsymbol{e}^{d+1}=$ $(0, \ldots, 0)$ for convenience. Let $v_{d+1}=0$.

(1). For $l=1, \ldots, d+1$ we have

$$
J_{v+\boldsymbol{e}^{l}}^{\prime}(x ; \boldsymbol{\varsigma})=\varsigma_{l} i(d+1) J_{\boldsymbol{v}+\boldsymbol{e}^{l-1}}(x ; \boldsymbol{\varsigma})-\frac{\Lambda_{d-l+1}(\boldsymbol{v})+d-l+1}{x} J_{\boldsymbol{v}+\boldsymbol{e}^{l}}(x ; \boldsymbol{\varsigma}),
$$

with

$$
\Lambda_{m}(\boldsymbol{v})=-\sum_{k=1}^{d} v_{k}+(d+1) v_{d-m+1}, \quad m=0, \ldots, d .
$$

(2). For $0 \leqslant j \leqslant k \leqslant d+1$ define

$$
U_{k, j}(\boldsymbol{v})= \begin{cases}1, & \text { if } j=k, \\ -\left(\Lambda_{j}(\boldsymbol{v})+k-1\right) U_{k-1, j}(\boldsymbol{v})+U_{k-1, j-1}(\boldsymbol{v}), & \text { if } 0 \leqslant j \leqslant k-1,\end{cases}
$$

\footnotetext{
III Added in proof: This problem was solved by Zhaolin Li, an undergraduate student at Zhejiang University.
} 
with the notation $U_{k,-1}(v)=0$, and

$$
S_{0}(\boldsymbol{s})=+, \quad S_{j}(\boldsymbol{s})=\prod_{m=0}^{j-1} \varsigma_{d-m+1} \text { for } j=1, \ldots, d+1 .
$$

Then

$$
\boldsymbol{J}_{\boldsymbol{v}}^{(k)}(x ; \boldsymbol{\varsigma})=\sum_{j=0}^{k} S_{j}(\boldsymbol{\varsigma})(i(d+1))^{j} U_{k, j}(\boldsymbol{v}) x^{j-k} J_{\boldsymbol{v}+\boldsymbol{e}^{d-j+1}}(x ; \boldsymbol{\varsigma}) .
$$

Proof. By (10.3) and 10.1,

$$
\begin{aligned}
& J_{v+\boldsymbol{e}^{l}}^{\prime}(x ; \boldsymbol{\varsigma})=\varsigma_{d+1} i(d+1) J_{v+\boldsymbol{e}^{l}+\boldsymbol{e}^{d}}(x ; \boldsymbol{\varsigma})+\frac{\sum_{k=1}^{d} v_{k}+l}{x} J_{\boldsymbol{v}+\boldsymbol{e}^{l}}(x ; \boldsymbol{\varsigma}) \\
& =i(d+1)\left(-\frac{v_{l}+1}{i x} J_{\boldsymbol{v}+\boldsymbol{e}^{l}}(x ; \boldsymbol{S})+\varsigma_{l} J_{\boldsymbol{v}+\boldsymbol{e}^{l-1}}(x ; \boldsymbol{\varsigma})\right)+\frac{\sum_{k=1}^{d} v_{k}+l}{x} J_{\boldsymbol{v}+\boldsymbol{e}^{l}}(x ; \boldsymbol{\varsigma}) \\
& =\varsigma_{l} i(d+1) J_{v+\boldsymbol{e}^{l-1}}(x ; \boldsymbol{\varsigma})+\frac{\sum_{k=1}^{d} v_{k}-(d+1) v_{l}+l-d-1}{x} J_{v+\boldsymbol{e}^{l}}(x ; \boldsymbol{\varsigma}) .
\end{aligned}
$$

This proves (10.4).

(10.5) is trivial when $k=0$. Suppose that $k \geqslant 1$ and that $(10.5)$ is already proven for $k-1$. The inductive hypothesis and (10.4) imply

$$
\begin{aligned}
J_{\boldsymbol{v}}^{(k)}(x ; \boldsymbol{\varsigma})= & \sum_{j=0}^{k-1} S_{j}(\boldsymbol{S})(i(d+1))^{j} U_{k-1, j}(\boldsymbol{v}) x^{j-k+1} \\
& \left((j-k+1) x^{-1} \boldsymbol{J}_{\boldsymbol{v}+\boldsymbol{e}^{d-j+1}}(x ; \boldsymbol{\varsigma})\right. \\
& \left.\quad+\boldsymbol{S}_{d-j+1} i(d+1) J_{\boldsymbol{v}+\boldsymbol{e}^{d-j}}(x ; \boldsymbol{s})-\left(\Lambda_{j}(\boldsymbol{v})+j\right) x^{-1} \boldsymbol{J}_{\boldsymbol{v}+\boldsymbol{e}^{d-j+1}}(x ; \boldsymbol{\varsigma})\right) \\
= & -\sum_{j=0}^{k-1} S_{j}(\boldsymbol{S})(i(d+1))^{j} U_{k-1, j}(\boldsymbol{v})\left(\Lambda_{j}(\boldsymbol{v})+k-1\right) x^{j-k} J_{\boldsymbol{v}+\boldsymbol{e}^{d-j+1}}(x ; \boldsymbol{\varsigma}) \\
& +\sum_{j=1}^{k} S_{j-1}(\boldsymbol{S}) \boldsymbol{S}_{d-j+2}(i(d+1))^{j} U_{k-1, j-1}(\boldsymbol{v}) x^{j-k} J_{\boldsymbol{v}+\boldsymbol{e}^{d-j+1}}(x ; \boldsymbol{\varsigma}) .
\end{aligned}
$$

Then 10.5 follows from the definitions of $U_{k, j}(\boldsymbol{v})$ and $S_{j}(\boldsymbol{\varsigma})$.

Q.E.D.

Lemma 10.1 (2) may be recapitulated as

$$
X_{v}(x ; \boldsymbol{s})=D(x)^{-1} U(\boldsymbol{v}) D(x) S(\boldsymbol{\varsigma}) Y_{v}(x ; \boldsymbol{\varsigma}),
$$

where $X_{\boldsymbol{v}}(x ; \boldsymbol{\varsigma})=\left(J_{\boldsymbol{v}}^{(k)}(x ; \boldsymbol{\varsigma})\right)_{k=0}^{d+1}$ and $Y_{\boldsymbol{v}}(x ; \boldsymbol{\varsigma})=\left(J_{\boldsymbol{v}+\boldsymbol{e}^{d-j+1}}(x ; \boldsymbol{\varsigma})\right)_{j=0}^{d+1}$ are column vectors of functions, $S(\boldsymbol{s})=\operatorname{diag}\left(S_{j}(\boldsymbol{S})(i(d+1))^{j}\right)_{j=0}^{d+1}$ and $D(x)=\operatorname{diag}\left(x^{j}\right)_{j=0}^{d+1}$ are diagonal matrices, and $U(v)$ is the lower triangular unipotent $(d+2) \times(d+2)$ matrix whose $(k+1, j+1)$-th entry is equal to $U_{k, j}(v)$. The inverse matrix $U(v)^{-1}$ is again a lower triangular unipotent matrix. Let $V_{k, j}(v)$ denote the $(k+1, j+1)$-th entry of $U(v)^{-1}$. It is evident that $V_{k, j}(\boldsymbol{v})$ is a polynomial in $\boldsymbol{v}$ of degree $k-j$ and integral coefficients.

Observe that $J_{v+e^{d+1}}(x ; \boldsymbol{s})=J_{v+e^{0}}(x ; \boldsymbol{s})=J_{v}(x ; \boldsymbol{s})$. Therefore, 10.6 implies that $J_{v}(x ; \boldsymbol{\varsigma})$ satisfies the following linear differential equation of order $d+1$

$$
\sum_{j=1}^{d+1} V_{d+1, j}(\boldsymbol{v}) x^{j-d-1} w^{(j)}+\left(V_{d+1,0}(\boldsymbol{v}) x^{-d-1}-S_{d+1}(\boldsymbol{\varsigma})(i(d+1))^{d+1}\right) w=0 .
$$




\subsection{Calculations of the Coefficients in the Differential Equations.}

Definition 10.2. Let $\boldsymbol{\Lambda}=\left\{\Lambda_{m}\right\}_{m=0}^{\infty}$ be a sequence of complex numbers.

(1). For $k, j \geqslant-1$ inductively define a double sequence of polynomials $U_{k, j}(\boldsymbol{\Lambda})$ in $\boldsymbol{\Lambda}$ by the initial conditions

$$
U_{-1,-1}(\boldsymbol{\Lambda})=1, \quad U_{k,-1}(\boldsymbol{\Lambda})=U_{-1, j}(\boldsymbol{\Lambda})=0 \text { if } k, j \geqslant 0,
$$

and the recurrence relation

$$
U_{k, j}(\boldsymbol{\Lambda})=-\left(\Lambda_{j}+k-1\right) U_{k-1, j}(\boldsymbol{\Lambda})+U_{k-1, j-1}(\boldsymbol{\Lambda}), \quad k, j \geqslant 0 .
$$

(2). For $j, m \geqslant-1$ with $(j, m) \neq(-1,-1)$ define a double sequence of integers $A_{j, m}$ by the initial conditions

$$
A_{-1,0}=1, \quad A_{-1, m}=A_{j,-1}=0 \text { if } m \geqslant 1, j \geqslant 0,
$$

and the recurrence relation

$$
A_{j, m}=j A_{j, m-1}+A_{j-1, m}, \quad j, m \geqslant 0 .
$$

(3). For $k, m \geqslant 0$ we define $\sigma_{k, m}(\boldsymbol{\Lambda})$ to be the elementary symmetric polynomial in $\Lambda_{0}, \ldots, \Lambda_{k}$ of degree $m$, with the convention that $\sigma_{k, m}(\boldsymbol{\Lambda})=0$ if $m \geqslant k+2$. Moreover, we denote

$$
\sigma_{-1,0}(\boldsymbol{\Lambda})=1, \quad \sigma_{k,-1}(\boldsymbol{\Lambda})=\sigma_{-1, m}(\boldsymbol{\Lambda})=0 \text { if } k \geqslant-1, m \geqslant 1 .
$$

Observe that, with the above notations as initial conditions, $\sigma_{k, m}(\boldsymbol{\Lambda})$ may also be inductively defined by the recurrence relation

$$
\sigma_{k, m}(\boldsymbol{\Lambda})=\Lambda_{k} \sigma_{k-1, m-1}(\boldsymbol{\Lambda})+\sigma_{k-1, m}(\boldsymbol{\Lambda}), \quad k, m \geqslant 0 .
$$

(4). For $k \geqslant 0, j \geqslant-1$ define

$$
V_{k, j}(\boldsymbol{\Lambda})= \begin{cases}0, & \text { if } j>k, \\ \sum_{m=0}^{k-j} A_{j, k-j-m} \sigma_{k-1, m}(\boldsymbol{\Lambda}), & \text { if } k \geqslant j .\end{cases}
$$

LEMMA 10.3. Let notations be as above.

(1). $U_{k, j}(\boldsymbol{\Lambda})$ is a polynomial in $\Lambda_{0}, \ldots, \Lambda_{j} . U_{k, j}(\boldsymbol{\Lambda})=0$ if $j>k$, and $U_{k, k}(\boldsymbol{\Lambda})=1$. $U_{k, 0}(\boldsymbol{\Lambda})=\left[-\Lambda_{0}\right]_{k}$ for $k \geqslant 0$.

(2). $A_{j, 0}=1$, and $A_{j, 1}=\frac{1}{2} j(j+1)$.

(3). $V_{k, j}(\boldsymbol{\Lambda})$ is a symmetric polynomial in $\Lambda_{0}, \ldots, \Lambda_{k-1} . V_{k, k}(\boldsymbol{\Lambda})=1 . V_{k,-1}(\boldsymbol{\Lambda})=0$ and $V_{k, k-1}(\boldsymbol{\Lambda})=\sigma_{k-1,1}(\boldsymbol{\Lambda})+\frac{1}{2} k(k-1)$ for $k \geqslant 0$.

(4). $V_{k, j}(\Lambda)$ satisfies the following recurrence relation

$$
V_{k, j}(\boldsymbol{\Lambda})=\left(\Lambda_{k-1}+j\right) V_{k-1, j}(\boldsymbol{\Lambda})+V_{k-1, j-1}(\boldsymbol{\Lambda}), \quad k \geqslant 1, j \geqslant 0 .
$$

Proof. (1-3) are evident from the definitions.

(4). 10.12 is obvious if $j \geqslant k$. If $k>j$, then the recurrence relations 10.10, 10.9 for $\sigma_{k, m}(\Lambda)$ and $A_{j, m}$, in conjunction with the definition 10.11 of $V_{k, j}(\Lambda)$, yield

$$
\begin{aligned}
V_{k, j}(\boldsymbol{\Lambda}) & =\sum_{m=0}^{k-j} A_{j, k-j-m} \sigma_{k-1, m}(\boldsymbol{\Lambda}) \\
& =\Lambda_{k-1} \sum_{m=1}^{k-j} A_{j, k-j-m} \sigma_{k-2, m-1}(\boldsymbol{\Lambda})+\sum_{m=0}^{k-j} A_{j, k-j-m} \sigma_{k-2, m}(\boldsymbol{\Lambda})
\end{aligned}
$$




$$
\begin{aligned}
& \text { 2. ANALYTIC THEORY OF BESSEL FUNCTIONS } \\
& \begin{aligned}
= & \Lambda_{k-1} \sum_{m=0}^{k-j-1} A_{j, k-j-m-1} \sigma_{k-2, m}(\boldsymbol{\Lambda}) \\
& +j \sum_{m=0}^{k-j-1} A_{j, k-j-m-1} \sigma_{k-2, m}(\boldsymbol{\Lambda})+\sum_{m=0}^{k-j} A_{j-1, k-j-m} \sigma_{k-2, m}(\boldsymbol{\Lambda}) \\
= & \left(\Lambda_{k-1}+j\right) V_{k-1, j}(\boldsymbol{\Lambda})+V_{k-1, j-1}(\boldsymbol{\Lambda}) .
\end{aligned}
\end{aligned}
$$

Q.E.D.

Lemma 10.4. For $k \geqslant 0$ and $j \geqslant-1$ such that $k \geqslant j$, we have

$$
\sum_{l=j}^{k} U_{k, l}(\boldsymbol{\Lambda}) V_{l, j}(\boldsymbol{\Lambda})=\delta_{k, j},
$$

where $\delta_{k, j}$ denotes Kronecker's delta symbol. Consequently,

$$
\sum_{l=j}^{k} V_{k, l}(\boldsymbol{\Lambda}) U_{l, j}(\boldsymbol{\Lambda})=\delta_{k, j} .
$$

Proof. (10.13) is obvious if either $k=j$ or $j=-1$. In the proof we may therefore assume that $k-1 \geqslant j \geqslant 0$ and that 10.13 is already proven for smaller values of $k-j$ as well as for smaller values of $j$ and the same $k-j$.

By the recurrence relations $10.8,10.12$ for $U_{k, j}(\Lambda)$ and $V_{k, j}(\Lambda)$ and the induction hypothesis,

$$
\begin{aligned}
& \sum_{l=j}^{k} U_{k, l}(\boldsymbol{\Lambda}) V_{l, j}(\boldsymbol{\Lambda}) \\
= & -\sum_{l=j}^{k-1}\left(k-1+\Lambda_{l}\right) U_{k-1, l}(\boldsymbol{\Lambda}) V_{l, j}(\boldsymbol{\Lambda})+\sum_{l=j}^{k} U_{k-1, l-1}(\boldsymbol{\Lambda}) V_{l, j}(\boldsymbol{\Lambda}) \\
= & -(k-1) \delta_{k-1, j}-\sum_{l=j}^{k-1} \Lambda_{l} U_{k-1, l}(\boldsymbol{\Lambda}) V_{l, j}(\boldsymbol{\Lambda})+\sum_{l=j+1}^{k} \Lambda_{l-1} U_{k-1, l-1}(\boldsymbol{\Lambda}) V_{l-1, j}(\boldsymbol{\Lambda}) \\
& +j \sum_{l=j+1}^{k} U_{k-1, l-1}(\boldsymbol{\Lambda}) V_{l-1, j}(\boldsymbol{\Lambda})+\sum_{l=j}^{k} U_{k-1, l-1}(\boldsymbol{\Lambda}) V_{l-1, j-1}(\boldsymbol{\Lambda}) \\
= & -(k-1) \delta_{k-1, j}+0+j \delta_{k-1, j}+\delta_{k-1, j-1}=0 .
\end{aligned}
$$

This completes the proof of 10.13.

Q.E.D.

Finally, we have the following explicit formulae for $A_{j, m}$.

Lemma 10.5. We have $A_{0,0}=1, A_{0, m}=0$ if $m \geqslant 1$, and

$$
A_{j, m}=\sum_{r=1}^{j} \frac{(-)^{j-r} r^{m+j}}{r !(j-r) !} \text { if } j \geqslant 1, m \geqslant 0 .
$$

Proof. It is easily seen that $A_{0,0}=1$ and $A_{0, m}=0$ if $m \geqslant 1$.

It is straightforward to verify that the sequence given by 10.15 satisfies the recurrence relation 10.9), so it is left to show that 10.15 holds true for $m=0$. Initially, $A_{j, 0}=1$, and hence one must verify

$$
\sum_{r=1}^{j} \frac{(-)^{j-r} r^{j}}{r !(j-r) !}=1
$$


This however follows from considering all the identities obtained by differentiating the following binomial identity up to $j$ times and then evaluating at $x=1$,

$$
(x-1)^{j}-(-1)^{j}=j ! \sum_{r=1}^{j} \frac{(-1)^{j-r}}{r !(j-r) !} x^{r} .
$$

Q.E.D.

10.4. Conclusion. We first observe that, when $0 \leqslant j \leqslant k \leqslant d+1$, both $U_{k, j}(\boldsymbol{\Lambda})$ and $V_{k, j}(\boldsymbol{\Lambda})$ are polynomials in $\Lambda_{0}, \ldots, \Lambda_{d}$ according to Lemma $10.3(1,3)$. If one puts $\Lambda_{m}=\Lambda_{m}(\boldsymbol{v})$ for $m=0, \ldots, d$, then $U_{k, j}(\boldsymbol{v})=U_{k, j}(\boldsymbol{\Lambda})$. It follows from Lemma 10.4 that $V_{k, j}(\boldsymbol{v})=V_{k, j}(\boldsymbol{\Lambda})$. Moreover, the relations $v_{l}=\lambda_{l}-\lambda_{d+1}, l=1, \ldots, d$, along with the assumption $\sum_{l=1}^{d+1} \lambda_{l}=0$, yields

$$
\Lambda_{m}(\boldsymbol{v})=(d+1) \lambda_{d-m+1} .
$$

Now we can reformulate (10.7) in the following theorem.

THEOREM 10.6. The Bessel function $J(x ; \boldsymbol{s}, \lambda)$ satisfies the following linear differential equation of order $d+1$

$$
\sum_{j=1}^{d+1} V_{d+1, j}(\lambda) x^{j} w^{(j)}+\left(V_{d+1,0}(\lambda)-S_{d+1}(\boldsymbol{\varsigma})(i(d+1))^{d+1} x^{d+1}\right) w=0,
$$

where

$$
S_{d+1}(\boldsymbol{S})=\prod_{l=1}^{d+1} \varsigma_{l}, \quad V_{d+1, j}(\lambda)=\sum_{m=0}^{d-j+1} A_{j, d-j-m+1}(d+1)^{m} \sigma_{m}(\lambda),
$$

$\sigma_{m}(\lambda)$ denotes the elementary symmetric polynomial in $\lambda$ of degree $m$, with $\sigma_{1}(\lambda)=0$, and $A_{j, m}$ is recurrently defined in Definition 10.2 (3) and explicitly given in Lemma 10.5 We shall call the equation (10.16) a Bessel equation of index $\lambda$, or simply a Bessel equation if the index $\lambda$ is given.

For a given index $\lambda, 10.16$ only provides two Bessel equations. The sign $S_{d+1}(\boldsymbol{s})$ determines which one of the two Bessel equations a Bessel function $J(x ; \boldsymbol{\varsigma}, \lambda)$ satisfies.

Definition 10.7. We call $S_{d+1}(\boldsymbol{s})=\prod_{l=1}^{d+1} \varsigma_{l}$ the sign of the Bessel function $J(x ; \boldsymbol{s}, \lambda)$ as well as the Bessel equation satisfied by $J(x ; \boldsymbol{\varsigma}, \lambda)$.

Finally, we collect some simple facts on $V_{d+1, j}(\lambda)$ in the following lemma, which will play important roles later in the study of Bessel equations. See (10.14) in Lemma 10.4 and Lemma 10.3 (3).

Lemma 10.8. We have

(1). $\sum_{j=0}^{d+1} V_{d+1, j}(\lambda)\left[-(d+1) \lambda_{d+1}\right]_{j}=0$.

(2). $V_{d+1, d}(\lambda)=\frac{1}{2} d(d+1)$.

Remark 10.9. If we define

$$
\boldsymbol{J}(x ; \boldsymbol{s}, \boldsymbol{\lambda})=J\left((d+1)^{-1} x ; \boldsymbol{s},(d+1)^{-1} \lambda\right),
$$

then this normalized Bessel function satisfies a differential equation with coefficients free of powers of $(d+1)$, that is,

$$
\sum_{j=1}^{d+1} \boldsymbol{V}_{d+1, j}(\boldsymbol{\lambda}) x^{j} w^{(j)}+\left(\boldsymbol{V}_{d+1,0}(\boldsymbol{\lambda})-S_{d+1}(\boldsymbol{\varsigma}) i^{d+1} x^{d+1}\right) w=0,
$$


with

$$
\boldsymbol{V}_{d+1, j}(\boldsymbol{\lambda})=\sum_{m=0}^{d-j+1} A_{j, d-j-m+1} \sigma_{m}(\boldsymbol{\lambda}) .
$$

In particular, if $d=1, \lambda=(\lambda,-\lambda)$, then the two normalized Bessel equations are

$$
x^{2} \frac{d^{2} w}{d x^{2}}+x \frac{d w}{d x}+\left(-\lambda^{2} \pm x^{2}\right) w=0 .
$$

These are exactly the Bessel equation and the modified Bessel equation of index $\lambda$.

\section{Bessel Equations}

The theory of linear ordinary differential equations with analytic coefficients ${ }^{\mathrm{IV}}$ will be employed in this section to study Bessel equations.

Subsequently, we shall use $z$ instead of $x$ to indicate complex variable. For $\varsigma \in\{+,-\}$ and $\lambda \in \mathbb{L}^{n-1}$, we introduce the Bessel differential operator

$$
\nabla_{\varsigma, \lambda}=\sum_{j=1}^{n} V_{n, j}(\lambda) z^{j} \frac{d^{j}}{d z^{j}}+V_{n, 0}(\lambda)-\varsigma(i n)^{n} z^{n} .
$$

The Bessel equation of index $\lambda$ and sign $\varsigma$ may be written as

$$
\nabla_{\varsigma, \lambda}(w)=0 \text {. }
$$

Its corresponding system of differential equations is given by

$$
w^{\prime}=B(z ; \varsigma, \lambda) w
$$

with

$$
B(z ; \varsigma, \lambda)=\left(\begin{array}{ccccc}
0 & 1 & 0 & \cdots & 0 \\
0 & 0 & 1 & \cdots & 0 \\
\vdots & \vdots & \vdots & \ddots & \vdots \\
0 & 0 & \cdots & \cdots & 1 \\
-V_{n, 0}(\lambda) z^{-n}+\varsigma(i n)^{n} & -V_{n, 1}(\lambda) z^{-n+1} & \cdots & \cdots & -V_{n, n-1}(\lambda) z^{-1}
\end{array}\right) .
$$

We shall study Bessel equations on the Riemann surface $\mathbb{U}$ associated with $\log z$, that is, the universal cover of $\mathbb{C} \backslash\{0\}$. Each element in $\mathbb{U}$ is represented by a pair $(x, \omega)$ with modulus $x \in \mathbb{R}_{+}$and argument $\omega \in \mathbb{R}$, and will be denoted by $z=x e^{i \omega}=e^{\log x+i \omega}$ with some ambiguity. Conventionally, define $z^{\lambda}=e^{\lambda \log z}$ for $z \in \mathbb{U}, \lambda \in \mathbb{C}, \bar{z}=e^{-\log z}$, and moreover let $1=e^{0},-1=e^{\pi i}$ and $\pm i=e^{ \pm \frac{1}{2} \pi i}$.

First of all, since Bessel equations are nonsingular on $\mathbb{U}$, all the solutions of Bessel equations are analytic on $\mathbb{U}$.

Each Bessel equation has only two singularities at $z=0$ and $z=\infty$. According to the classification of singularities, 0 is a regular singularity, so the Frobenius method gives rise to solutions of Bessel equations developed in series of ascending powers of $z$, or possibly logarithmic sums of this kind of series, whereas $\infty$ is an irregular singularity of rank one, and therefore one may find certain formal solutions that are the asymptotic expansions of some actual solutions of Bessel equations. Accordingly, there are two kinds of Bessel functions arising as solutions of Bessel equations.

Finally, a simple but important observation is as follows.

${ }^{I V}[\mathbf{C L}$ Chapter 4, 5] and [Was Chapter II-V] are the main references that we follow, and the reader is referred to these books for terminologies and definitions. 
Lemma 11.1. Let $\varsigma \in\{+,-\}$ and a be an integer. If $\varphi(z)$ is a solution of the Bessel equation of sign $\varsigma$, then $\varphi\left(e^{\pi i \frac{a}{n}} z\right)$ satisfies the Bessel equation of sign $(-)^{a} \varsigma$.

Variants of Lemma 11.1. Lemma 11.3, 11.10 and 11.21. will play important roles later in $\$ 12$ when we establish the connection formulae for various kinds of Bessel functions.

11.1. Bessel Functions of the First Kind. The indicial equation associated with $\nabla_{\varsigma, \lambda}$ is given as below,

$$
\sum_{j=0}^{n}[\rho]_{j} V_{n, j}(\lambda)=0
$$

Let $P_{\lambda}(\rho)$ denote the polynomial on the left of this equation. Lemma 10.8 (1) along with the symmetry of $V_{n, j}(\lambda)$ yields the following identity,

$$
\sum_{j=0}^{n}\left[-n \lambda_{l}\right]_{j} V_{n, j}(\lambda)=0
$$

for each $l=1, \ldots, n$. Therefore,

$$
P_{\lambda}(\rho)=\prod_{l=1}^{n}\left(\rho+n \lambda_{l}\right)
$$

Consider the formal series

$$
\sum_{m=0}^{\infty} c_{m} z^{\rho+m}
$$

where the index $\rho$ and the coefficients $c_{m}$, with $c_{0} \neq 0$, are to be determined. It is easy to see that

$$
\nabla_{\varsigma, \lambda} \sum_{m=0}^{\infty} c_{m} z^{\rho+m}=\sum_{m=0}^{\infty} c_{m} P_{\lambda}(\rho+m) z^{\rho+m}-\varsigma(\text { in })^{n} \sum_{m=0}^{\infty} c_{m} z^{\rho+m+n} .
$$

If the following equations are satisfied

$$
\begin{aligned}
& c_{m} P_{\lambda}(\rho+m)=0, \quad n>m \geqslant 1, \\
& c_{m} P_{\lambda}(\rho+m)-\varsigma(i n)^{n} c_{m-n}=0, \quad m \geqslant n,
\end{aligned}
$$

then

$$
\nabla_{\varsigma, \lambda} \sum_{m=0}^{\infty} c_{m} z^{\rho+m}=c_{0} P_{\lambda}(\rho) z^{\rho}
$$

Given $l \in\{1, \ldots, n\}$. Choose $\rho=-n \lambda_{l}$ and let $c_{0}=\prod_{k=1}^{n} \Gamma\left(\lambda_{k}-\lambda_{l}+1\right)^{-1}$. Suppose, for the moment, that no two components of $n \lambda$ differ by an integer. Then $P_{\lambda}\left(-n \lambda_{l}+m\right) \neq 0$ for any $m \geqslant 1$ and $c_{0} \neq 0$, and hence the system of equations 11.4 is uniquely solvable. It follows that

$$
\sum_{m=0}^{\infty} \frac{\left(\varsigma i^{n}\right)^{m} z^{n\left(-\lambda_{l}+m\right)}}{\prod_{k=1}^{n} \Gamma\left(\lambda_{k}-\lambda_{l}+m+1\right)}
$$

is a formal solution of the differential equation (11.2).

Now suppose that $\lambda \in \mathbb{L}^{n-1}$ is unrestricted. The series in 11.5 is absolutely convergent, compactly convergent with respect to $\lambda$, and hence gives rise to an analytic function of $z$ on the Riemann surface $\mathbb{U}$, as well as an analytic function of $\lambda$. We denote by $J_{l}(z ; \varsigma, \lambda)$ the analytic function given by the series (11.5) and call it a Bessel function of the first kind. It is evident that $J_{l}(z ; \varsigma, \lambda)$ is an actual solution of 11.2 . 
Definition 11.2. Let $\mathbb{D}^{n-1}$ denote the set of $\lambda \in \mathbb{L}^{n-1}$ such that no two components of $\lambda$ differ by an integer. We call an index $\lambda$ generic if $\lambda \in \mathbb{D}^{n-1}$.

When $\lambda \in \mathbb{D}^{n-1}$, all the $J_{l}(z ; \varsigma, \lambda)$ constitute a fundamental set of solutions, since the leading term in the expression 11.5 of $J_{l}(z ; \varsigma, \lambda)$ does not vanish. However, this is no longer the case if $\lambda \notin \mathbb{D}^{n-1}$. Indeed, if $\lambda_{l}-\lambda_{k}$ is an integer, $k \neq l$, then $J_{l}(z ; \varsigma, \lambda)=$ $\left(\varsigma i^{n}\right)^{\lambda_{l}-\lambda_{k}} J_{k}(z ; \varsigma, \lambda)$. There are other solutions arising as certain logarithmic sums of series of ascending powers of $z$. Roughly speaking, powers of $\log z$ may occur in some solutions. For more details the reader may consult [CL, \$4.8].

LEMMA 11.3. Let a be an integer. We have

$$
J_{l}\left(e^{\pi i \frac{a}{n}} z ; \varsigma, \lambda\right)=e^{-\pi i a \lambda_{l}} J_{l}\left(z ;(-)^{a} \varsigma, \lambda\right) .
$$

REMARK 11.4. If $n=2$, then we have the following formulae according to [Wat 3.1 (8), 3.7 (2)],

$$
\begin{array}{ll}
J_{1}(z ;+, \lambda,-\lambda)=J_{-2 \lambda}(2 z), & J_{2}(z ;+, \lambda,-\lambda)=J_{2 \lambda}(2 z), \\
J_{1}(z ;-, \lambda,-\lambda)=I_{-2 \lambda}(2 z), & J_{2}(z ;-, \lambda,-\lambda)=I_{2 \lambda}(2 z) .
\end{array}
$$

REMARK 11.5. Recall the definition of the generalized hypergeometric functions given by the series [Wat, §4.4]

$$
{ }_{p} F_{q}\left(\alpha_{1}, \ldots, \alpha_{p} ; \rho_{1}, \ldots, \rho_{q} ; z\right)=\sum_{m=0}^{\infty} \frac{\left(\alpha_{1}\right)_{m} \ldots\left(\alpha_{p}\right)_{m}}{m !\left(\rho_{1}\right)_{m} \ldots\left(\rho_{q}\right)_{m}} z^{m} .
$$

It is evident that each Bessel function $J_{l}(z ; \varsigma, \lambda)$ is closely related to a certain generalized hypergeometric function ${ }_{0} F_{n-1}$ as follows

$$
J_{l}(z ; \varsigma, \lambda)=\left(\prod_{k \neq l} \frac{z^{\lambda_{k}-\lambda_{l}}}{\Gamma\left(\lambda_{k}-\lambda_{l}+1\right)}\right) \cdot{ }_{0} F_{n-1}\left(\left\{\lambda_{k}-\lambda_{l}+1\right\}_{k \neq l} ; \varsigma i^{n} z^{n}\right) .
$$

11.2. The Analytic Continuation of $J(x ; \boldsymbol{s}, \lambda)$. In $\$ 3.1 .2$, from its Barnes integral representation, we have seen that $J(x ; \boldsymbol{\varsigma}, \lambda)$ admits a unique analytic continuation from $\mathbb{R}_{+}$onto $\mathbb{U}$. For any given $\lambda \in \mathbb{L}^{n-1}$, since $J(x ; \boldsymbol{s}, \lambda)$ satisfies the Bessel equation of sign $S_{n}(\boldsymbol{\varsigma})$, such an analytic continuation $J(z ; \boldsymbol{\varsigma}, \lambda)$ on $\mathbb{U}$ is also clear from the theory of differential equations. In the following, we shall make $J(z ; \boldsymbol{s}, \lambda)$ more precise in terms of Bessel functions of the first kind.

Recall the definition

$$
J(x ; \boldsymbol{\varsigma}, \lambda)=\frac{1}{2 \pi i} \int_{\mathcal{C}} G(s ; \boldsymbol{\varsigma}, \lambda) x^{-n s} d s, \quad x \in \mathbb{R}_{+},
$$

where $G(s ; \boldsymbol{s}, \lambda)=\prod_{k=1}^{n} \Gamma\left(s-\lambda_{k}\right) e\left(\frac{1}{4} \varsigma_{k}\left(s-\lambda_{k}\right)\right)$ and $\mathcal{C}$ is a suitable contour.

Let $\boldsymbol{\varsigma}=S_{n}(\boldsymbol{\varsigma})$. For the moment, let us assume that $\lambda$ is generic. For $l=1, \ldots, n$ and $m=0,1,2, \ldots, G(s ; \boldsymbol{s}, \lambda)$ has a simple pole at $\lambda_{l}-m$ with residue

$$
\begin{aligned}
& (-)^{m} \frac{1}{m !} e\left(\frac{\sum_{k=1}^{n} \varsigma_{k}\left(\lambda_{l}-\lambda_{k}-m\right)}{4}\right) \prod_{k \neq l} \Gamma\left(\lambda_{l}-\lambda_{k}-m\right)= \\
& \pi^{n-1} e\left(-\frac{\sum_{k=1}^{n} \varsigma_{k} \lambda_{k}}{4}\right) e\left(\frac{\sum_{k=1}^{n} \varsigma_{k} \lambda_{l}}{4}\right)\left(\prod_{k \neq l} \frac{1}{\sin \left(\pi\left(\lambda_{l}-\lambda_{k}\right)\right)}\right) \frac{\left(s i^{n}\right)^{m}}{\prod_{k=1}^{n} \Gamma\left(\lambda_{k}-\lambda_{l}+m+1\right)} .
\end{aligned}
$$

Here we have used Euler's reflection formula for the Gamma function. Applying Cauchy's residue theorem, $J(x ; \boldsymbol{s}, \lambda)$ is developed into an absolutely convergent series on shifting the 
contour $\mathcal{C}$ far left, and, in view of (11.5), we obtain

$$
J(z ; \boldsymbol{\varsigma}, \lambda)=\pi^{n-1} E(\boldsymbol{\varsigma}, \lambda) \sum_{l=1}^{n} E_{l}(\boldsymbol{\varsigma}, \lambda) S_{l}(\lambda) J_{l}(z ; \varsigma, \lambda), \quad z \in \mathbb{U},
$$

with

$E(\boldsymbol{s}, \lambda)=e\left(-\frac{\sum_{k=1}^{n} \varsigma_{k} \lambda_{k}}{4}\right), E_{l}(\boldsymbol{s}, \boldsymbol{\lambda})=e\left(\frac{\sum_{k=1}^{n} \varsigma_{k} \lambda_{l}}{4}\right), S_{l}(\boldsymbol{\lambda})=\prod_{k \neq l} \frac{1}{\sin \left(\pi\left(\lambda_{l}-\lambda_{k}\right)\right)}$.

Because of the possible vanishing of $\sin \left(\pi\left(\lambda_{l}-\lambda_{k}\right)\right)$, the definition of $S_{l}(\lambda)$ may fail to make sense if $\lambda$ is not generic. In order to properly interpret (11.6) in the nongeneric case, one has to pass to the limit, that is,

$$
J(z ; \boldsymbol{\varsigma}, \lambda)=\pi^{n-1} E(\boldsymbol{\varsigma}, \lambda) \cdot \lim _{\substack{\lambda^{\prime} \rightarrow \lambda \\ \lambda^{\prime} \in \mathbb{D}^{n-1}}} \sum_{l=1}^{n} E_{l}\left(\boldsymbol{\varsigma}, \lambda^{\prime}\right) S_{l}\left(\boldsymbol{\lambda}^{\prime}\right) J_{l}\left(z ; \boldsymbol{\varsigma}, \lambda^{\prime}\right) .
$$

We recollect the definitions of $L_{ \pm}(\boldsymbol{\varsigma})$ and $n_{ \pm}(\boldsymbol{\varsigma})$ introduced in Proposition A.1.

Definition 11.6. Let $\boldsymbol{\varsigma} \in\{+,-\}^{n}$. We define $L_{ \pm}(\boldsymbol{\varsigma})=\left\{l: \boldsymbol{\varsigma}_{l}= \pm\right\}$ and $n_{ \pm}(\boldsymbol{\varsigma})=$ $\left|L_{ \pm}(\boldsymbol{\varsigma})\right|$. The pair of integers $\left(n_{+}(\boldsymbol{\varsigma}), n_{-}(\boldsymbol{\varsigma})\right)$ is called the signature of $\boldsymbol{\varsigma}$, as well as the signature of the Bessel function $J(z ; \boldsymbol{\varsigma}, \lambda)$.

With Definition 11.6, we reformulate (11.6, 11.7) in the following lemma.

Lemma 11.7. We have

$$
J(z ; \boldsymbol{s}, \lambda)=\pi^{n-1} E(\boldsymbol{s}, \lambda) \sum_{l=1}^{n} E_{l}(\boldsymbol{s}, \lambda) S_{l}(\lambda) J_{l}\left(z ;(-)^{n_{-}(\boldsymbol{\varsigma})}, \lambda\right),
$$

with $E(\boldsymbol{s}, \lambda)=e\left(-\frac{1}{4} \sum_{k \in L_{+}(\boldsymbol{s})}+\frac{1}{4} \sum_{k \in L_{-}(\boldsymbol{s})} \lambda_{k}\right), E_{l}(\boldsymbol{s}, \lambda)=e\left(\frac{1}{4}\left(n_{+}(\boldsymbol{s})-n_{-}(\boldsymbol{s})\right) \lambda_{l}\right)$ and $S_{l}(\lambda)=1 / \prod_{k \neq l} \sin \left(\pi\left(\lambda_{l}-\lambda_{k}\right)\right)$. When $\lambda$ is not generic, the right hand side is to be replaced by its limit.

Remark 11.8. In view of Proposition 4.4 and Remark 11.4. Lemma 11.7 is equivalent to the connection formulae in 0.5 , 0.6) (see [Wat 3.61(5, 6), 3.7 (6)]).

Remark 11.9. In the case when $\lambda=\frac{1}{n}\left(\frac{n-1}{2}, \ldots,-\frac{n-1}{2}\right)$, the formula in Lemma 11.7 amounts to splitting the Taylor series expansion of $e^{i n \xi(\boldsymbol{\xi}) x}$ in A.1 according to the congruence classes of indices modulo $n$. To see this, one requires the multiplicative formula of the Gamma function (A.2) as well as the trigonometric identity

$$
\prod_{k=1}^{n-1} \sin \left(\frac{k \pi}{n}\right)=\frac{n}{2^{n-1}} .
$$

Using Lemma 11.3 and 11.7, one proves the following lemma, which implies that the Bessel function $J(z ; \boldsymbol{\varsigma}, \lambda)$ is determined by its signature up to a constant multiple.

Lemma 11.10. Define $H^{ \pm}(z ; \lambda)=J(z ; \pm, \ldots, \pm, \lambda)$. Then

$$
J(z ; \boldsymbol{\varsigma}, \lambda)=e\left( \pm \frac{\sum_{l \in L_{\mp}(\boldsymbol{s})} \lambda_{l}}{2}\right) H^{ \pm}\left(e^{ \pm \pi i \frac{n_{\mp}(\boldsymbol{s})}{n}} z ; \lambda\right) .
$$


11.3. Asymptotics for Bessel Equations and Bessel Functions of the Second Kind. Subsequently, we proceed to investigate the asymptotics at infinity for Bessel equations.

Definition 11.11. For $\varsigma \in\{+,-\}$ and a positive integer $N$, we let $\mathbb{X}_{N}(\varsigma)$ denote the set of $N$-th roots of $\varsigma 1 \mathrm{~V}$

Before delving into our general study, let us first consider the prototypical example given in Proposition A.1

Proposition 11.12. For any $\xi \in \mathbb{X}_{2 n}(+)$, the function $z^{-\frac{n-1}{2}} e^{i n \xi z}$ is a solution of the Bessel equation of index $\frac{1}{n}\left(\frac{n-1}{2}, \ldots,-\frac{n-1}{2}\right)$ and sign $\xi^{n}$.

Proof. When $\mathfrak{I m} \xi \geqslant 0$, this can be seen from Proposition A.1 and Theorem 10.6 For arbitrary $\xi$, one makes use of Lemma 11.1 .

Q.E.D.

11.3.1. Formal Solutions of Bessel Equations at Infinity. Following [CL, Chapter 5], we shall consider the system of differential equations (11.3). We have

$$
B(\infty ; \varsigma, \lambda)=\left(\begin{array}{ccccc}
0 & 1 & 0 & \cdots & 0 \\
0 & 0 & 1 & \cdots & 0 \\
\vdots & \vdots & \vdots & \ddots & \vdots \\
0 & 0 & 0 & \cdots & 1 \\
\varsigma(i n)^{n} & 0 & \cdots & \cdots & 0
\end{array}\right)
$$

If one let $\mathbb{X}_{n}(\varsigma)=\left\{\xi_{1}, \ldots, \xi_{n}\right\}$, then the eigenvalues of $B(\infty ; \varsigma, \lambda)$ are $i n \xi_{1}, \ldots, i n \xi_{n}$. The conjugation by the following matrix diagonalizes $B(\infty ; \varsigma, \lambda)$,

$$
\begin{aligned}
T & =\frac{1}{n}\left(\begin{array}{cccc}
1 & \left(i n \xi_{1}\right)^{-1} & \ldots & \left(i n \xi_{1}\right)^{-n+1} \\
1 & \left(i n \xi_{2}\right)^{-1} & \ldots & \left(i n \xi_{2}\right)^{-n+1} \\
\cdots & \cdots & \ldots & \ldots \\
1 & \left(i n \xi_{n}\right)^{-1} & \ldots & \left(i n \xi_{n}\right)^{-n+1}
\end{array}\right) \\
T^{-1} & =\left(\begin{array}{cccc}
1 & 1 & \cdots & 1 \\
i n \xi_{1} & i n \xi_{2} & \cdots & i n \xi_{n} \\
\cdots & \cdots & \cdots & \cdots \\
\left(i n \xi_{1}\right)^{n-1} & \left(i n \xi_{2}\right)^{n-1} & \cdots & \left(i n \xi_{n}\right)^{n-1}
\end{array}\right) .
\end{aligned}
$$

Thus, the substitution $u=T w$ converts the system of differential equations 11.3 into

$$
u^{\prime}=A(z) u
$$

where $A(z)=T B(z ; \varsigma, \lambda) T^{-1}$ is a matrix of polynomials in $z^{-1}$ of degree $n$,

$$
A(z)=\sum_{j=0}^{n} z^{-j} A_{j}
$$

with

$$
\begin{aligned}
& A_{0}=\Delta=\operatorname{diag}\left(\operatorname{in} \xi_{l}\right)_{l=1}^{n}, \\
& A_{j}=-i^{-j+1} n^{-j} V_{n, n-j}(\lambda)\left(\xi_{k} \xi_{l}^{-j}\right)_{k, l=1}^{n}, j=1, \ldots, n .
\end{aligned}
$$

It is convenient to put $A_{j}=0$ if $j>n$. The dependence on $\varsigma, \lambda$ and the ordering of the eigenvalues have been suppressed in our notation for the interest of brevity.

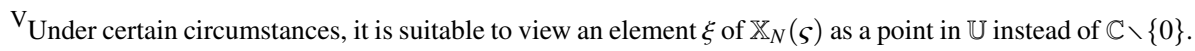
This however should be clear from the context.
} 
Suppose $\widehat{\Phi}$ is a formal solution matrix for 11.8 of the form

$$
\widehat{\Phi}(z)=P(z) z^{R} e^{Q z},
$$

where $P$ is a formal power series in $z^{-1}$,

$$
P(z)=\sum_{m=0}^{\infty} z^{-m} P_{m}
$$

and $R, Q$ are constant diagonal matrices. Since

$$
\widehat{\Phi}^{\prime}=P^{\prime} z^{R} e^{Q z}+z^{-1} P R z^{R} e^{Q z}+P z^{R} Q e^{Q z}=\left(P^{\prime}+z^{-1} P R+P Q\right) z^{R} e^{Q z},
$$

the differential equation 11.8 yields

$$
\sum_{m=0}^{\infty} z^{-m-1} P_{m}(R-m I)+\sum_{m=0}^{\infty} z^{-m} P_{m} Q=\left(\sum_{j=0}^{\infty} z^{-j} A_{j}\right)\left(\sum_{m=0}^{\infty} z^{-m} P_{m}\right),
$$

where $I$ denotes the identity matrix. Comparing the coefficients of various powers of $z^{-1}$, it follows that $\widehat{\Phi}$ is a formal solution matrix for 11.8 if and only if $R, Q$ and $P_{m}$ satisfy the following equations

$$
\begin{aligned}
P_{0} Q-\Delta P_{0} & =0 \\
P_{m+1} Q-\Delta P_{m+1} & =\sum_{j=1}^{m+1} A_{j} P_{m-j+1}+P_{m}(m I-R), \quad m \geqslant 0 .
\end{aligned}
$$

A solution of the first equation in 11.10 is given by

$$
Q=\Delta, \quad P_{0}=I .
$$

Using (11.11, the second equation in 11.10) for $m=0$ becomes

$$
P_{1} \Delta-\Delta P_{1}=A_{1}-R
$$

Since $\Delta$ is diagonal, the diagonal entries of the left side of (11.12) are zero, and hence the diagonal entries of $R$ must be identical with those of $A_{1}$. In view of $(11.9)$ and Lemma 10.8 (2), we have

$$
A_{1}=-\frac{1}{n} V_{n, n-1}(\lambda) \cdot\left(\xi_{k} \xi_{l}^{-1}\right)_{k, l=1}^{n}=-\frac{n-1}{2}\left(\xi_{k} \xi_{l}^{-1}\right)_{k, l=1}^{n},
$$

and therefore

$$
R=-\frac{n-1}{2} I
$$

Let $p_{1, k l}$ denote the $(k, l)$-th entry of $P_{1}$. It follows from 11.9 11.12 that

$$
\operatorname{in}\left(\xi_{l}-\xi_{k}\right) p_{1, k l}=-\frac{n-1}{2} \xi_{k} \xi_{l}^{-1}, \quad k \neq l
$$

The off-diagonal entries of $P_{1}$ are uniquely determined by (11.14). Therefore, a solution of $(11.12)$ is

$$
P_{1}=D_{1}+P_{1}^{o},
$$

where $D_{1}$ is any diagonal matrix and $P_{1}^{o}$ is the matrix with diagonal entries zero and $(k, l)$ th entry $p_{1, k l}, k \neq l$. To determine $D_{1}$, one resorts to the second equation in 11.10 for $m=1$, which, in view of 11.11, 11.13, 11.15, may be written as

$$
P_{2} \Delta-\Delta P_{2}-\left(A_{1}+\frac{n-1}{2}\right) D_{1}-\frac{n+1}{2} P_{1}^{o}=A_{1} P_{1}^{o}+A_{2}+D_{1} .
$$


The matrix on the left side has zero diagonal entries. It follows that $D_{1}$ must be equal to the diagonal part of $-A_{1} P_{1}^{o}-A_{2}$.

In general, using $11.11,11.13$, the second equation in 11.10 may be written as

$$
P_{m+1} \Delta-\Delta P_{m+1}=\sum_{j=1}^{m+1} A_{j} P_{m-j+1}+\left(m+\frac{n-1}{2}\right) P_{m}, \quad m \geqslant 0 .
$$

Applying 11.16, an induction on $m$ implies that

$$
P_{m}=D_{m}+P_{m}^{o}, \quad m \geqslant 1
$$

where $D_{m}$ and $P_{m}^{o}$ are inductively defined as follows. Put $D_{0}=I$. Let $m D_{m}$ be the diagonal part of

$$
-\sum_{j=2}^{m+1} A_{j} D_{m-j+1}-\sum_{j=1}^{m} A_{j} P_{m-j+1}^{o},
$$

and let $P_{m+1}^{o}$ be the matrix with diagonal entries zero such that $P_{m+1}^{o} \Delta-\Delta P_{m+1}^{o}$ is the off-diagonal part of

$$
\sum_{j=1}^{m+1} A_{j} D_{m-j+1}+\sum_{j=1}^{m} A_{j} P_{m-j+1}^{o}+\left(m+\frac{n-1}{2}\right) P_{m}^{o} .
$$

In this way, an inductive construction of the formal solution matrix of 11.8$)$ is completed for the given initial choices $Q=\Delta, P_{0}=I$.

With the observations that $A_{j}$ is of degree $j$ in $\lambda$ for $j \geqslant 2$ and that $A_{1}$ is constant, we may show the following lemma using an inductive argument.

Lemma 11.13. The entries of $P_{m}$ are symmetric polynomials in $\lambda$. If $m \geqslant 1$, then the off-diagonal entries of $P_{m}$ have degree at most $2 m-2$, whereas the degree of each diagonal entry is exactly $2 m$.

The first row of $T^{-1} \widehat{\Phi}$ constitutes a fundamental system of formal solutions of the Bessel equation (11.2). Some calculations yield the following proposition, where for derivatives of order higher than $n-1$ the differential equation $(11.2)$ is applied.

Proposition 11.14. Let $\varsigma \in\{+,-\}$ and $\xi \in \mathbb{X}_{n}(\varsigma)$. There exists a unique sequence of symmetric polynomials $B_{m}(\lambda ; \xi)$ in $\lambda$ of degree $2 m$ and coefficients depending only on $m, \xi$ and $n$, normalized so that $B_{0}(\lambda ; \xi)=1$, such that

$$
e^{i n \xi z} z^{-\frac{n-1}{2}} \sum_{m=0}^{\infty} B_{m}(\lambda ; \xi) z^{-m}
$$

is a formal solution of the Bessel equation of sign $\varsigma$ (11.2). We shall denote the formal series in 11.17 by $\widehat{J}(z ; \lambda ; \xi)$. Moreover, the $j$-th formal derivative $\widehat{J}^{(j)}(z ; \lambda ; \xi)$ is also of the form as (11.17), but with coefficients depending on $j$ as well.

REMARK 11.15. The above arguments are essentially adapted from the proof of $[\mathbf{C L}$, Chapter 5, Theorem 2.1]. This construction of the formal solution and Lemma 11.13 will be required later in $\S 11.4$ for the error analysis.

However, This method is not the best for the actual computation of the coefficients $B_{m}(\lambda ; \xi)$. We may derive the recurrent relations for $B_{m}(\lambda ; \xi)$ by a more direct but less suggestive approach as follows. 
The substitution $w=e^{i n \xi z} z^{-\frac{n-1}{2}} u$ transforms the Bessel equation 11.2 into

$$
\sum_{j=0}^{n} W_{j}(z ; \lambda) u^{(j)}=0
$$

where $W_{j}(z ; \lambda)$ is a polynomial in $z^{-1}$ of degree $n-j$,

$$
W_{j}(z ; \lambda)=\sum_{k=0}^{n-j} W_{j, k}(\lambda) z^{-k}
$$

with

$$
\begin{aligned}
W_{0,0}(\lambda) & =(i n \xi)^{n}-\varsigma(i n)^{n}=0, \\
W_{j, k}(\lambda) & =\frac{(i n \xi)^{n-j-k}}{j !(n-j-k) !} \sum_{r=0}^{k} \frac{(n-r) !}{(k-r) !}\left[-\frac{n-1}{2}\right]_{k-r} V_{n, n-r}(\lambda), \quad(j, k) \neq(0,0) .
\end{aligned}
$$

We have

$$
W_{0,1}(\lambda)=(i n \xi)^{n-1}\left(n\left(-\frac{n-1}{2}\right) V_{n, n}(\lambda)+V_{n, n-1}(\lambda)\right)=0,
$$

but $W_{1,0}(\lambda)=n(i n \xi)^{n-1}$ is nonzero. Some calculations show that $B_{m}(\lambda ; \xi)$ satisfy the following recurrence relations

$$
(m-1) W_{1,0}(\lambda) B_{m-1}(\lambda ; \xi)=\sum_{k=2}^{\min \{m, n\}} B_{m-k}(\lambda ; \xi) \sum_{j=0}^{k} W_{j, k-j}(\lambda)[k-m]_{j}, \quad m \geqslant 2 .
$$

If $n=2$, for a fourth root of unity $\xi= \pm 1, \pm i$ one may calculate in this way to obtain

$$
B_{m}(\lambda,-\lambda ; \xi)=\frac{\left(\frac{1}{2}-2 \lambda\right)_{m}\left(\frac{1}{2}+2 \lambda\right)_{m}}{(4 i \xi)^{m} m !} .
$$

11.3.2. Bessel Functions of the Second Kind. Bessel functions of the second kind are solutions of Bessel equations defined according to their asymptotic expansions at infinity. We shall apply several results in the asymptotic theory of ordinary differential equations from [Was, Chapter IV].

Firstly, [Was, Theorem 12.3] implies the following lemma.

Lemma 11.16 (Existence of solutions). Let $\varsigma \in\{+,-\}, \xi \in \mathbb{X}_{n}(\varsigma)$, and $\mathbb{S} \subset \mathbb{U}$ be an open sector with vertex at the origin and a positive central angle not exceeding $\pi$. Then there exists a solution of the Bessel equation of sign $\varsigma$ (11.2) that has the asymptotic expansion $\widehat{J}(z ; \lambda ; \xi)$ defined in 11.17 on $\mathbb{S}$. Moreover, each derivative of this solution has the formal derivative of $\widehat{J}(z ; \lambda ; \xi)$ of the same order as its asymptotic expansion.

For two distinct $\xi, \xi^{\prime} \in \mathbb{X}_{n}(\varsigma)$, the ray emitted from the origin on which

$$
\mathfrak{R e}\left(\left(i \xi-i \xi^{\prime}\right) z\right)=-\mathfrak{I m}\left(\left(\xi-\xi^{\prime}\right) z\right)=0
$$

is called a separation ray.

We first consider the case $n=2$. It is clear that the separation rays constitute either the real or the imaginary axis and thus separate $\mathbb{C} \backslash\{0\}$ into two half-planes. Accordingly, we define $\mathbb{S}_{ \pm 1}=\{z: \pm \mathfrak{I m} z>0\}$ and $\mathbb{S}_{ \pm i}=\{z: \pm \mathfrak{R e} z>0\}$.

In the case $n \geqslant 3$, there are $2 n$ distinct separation rays in $\mathbb{C} \backslash\{0\}$ given by the equations

$$
\arg z=\arg \left(i \xi^{\prime}\right), \quad \xi^{\prime} \in \mathbb{X}_{2 n}(+) .
$$


These separation rays divide $\mathbb{C} \backslash\{0\}$ into $2 n$ many open sectors

$$
\mathbb{S}_{\xi}^{ \pm}=\left\{z: 0< \pm(\arg z-\arg (i \bar{\xi}))<\frac{\pi}{n}\right\}, \quad \xi \in \mathbb{X}_{n}(\varsigma) .
$$

In both sectors $\mathbb{S}_{\xi}^{+}$and $\mathbb{S}_{\xi}^{-}$we have

$$
\mathfrak{R e}(i \xi z)<\mathfrak{R e}\left(i \xi^{\prime} z\right) \text { for all } \xi^{\prime} \in \mathbb{X}_{n}(S), \xi^{\prime} \neq \xi
$$

Let $\mathbb{S}_{\xi}$ be the sector on which 11.19 is satisfied. It is evident that

$$
\mathbb{S}_{\xi}=\left\{z:|\arg z-\arg (i \bar{\xi})|<\frac{\pi}{n}\right\} .
$$

Lemma 11.17. Let $\varsigma \in\{+,-\}$ and $\xi \in \mathbb{X}_{n}(\varsigma)$.

(1. Existence of asymptotics). If $n \geqslant 3$, on the sector $\mathbb{S}_{\xi}^{ \pm}$, all the solutions of the Bessel equation of sign $\varsigma$ have asymptotic representation a multiple of $\widehat{J}\left(z ; \lambda ; \xi^{\prime}\right)$ for some $\xi^{\prime} \in \mathbb{X}_{n}(\varsigma)$. If $n=2$, the same assertion is true with $\mathbb{S}_{\xi}^{ \pm}$replaced by $\mathbb{S}_{\xi}$.

(2. Uniqueness of the solution). There is a unique solution of the Bessel equation of sign $\varsigma$ that possesses $\widehat{J}(z ; \lambda ; \xi)$ as its asymptotic expansion on $\mathbb{S}_{\xi}$ or any of its open subsector, and we shall denote this solution by $J(z ; \lambda ; \xi)$. Moreover, $J^{(j)}(z ; \lambda ; \xi) \sim \widehat{J}^{(j)}(z ; \lambda ; \xi)$ on $\mathbb{S}_{\xi}$ for any $j \geqslant 0$.

Proof. (1) follows directly from [Was, Theorem 15.1].

For $n=2$, since (11.19) holds for the sector $\mathbb{S}_{\xi}$, (2) is true according to Was, Corollary to Theorem 15.3]. Similarly, if $n \geqslant 3$, (2) is true with $\mathbb{S}_{\xi}$ replaced by $\mathbb{S}_{\xi}^{ \pm}$. Thus there exists a unique solution of the Bessel equation of sign $\varsigma$ possessing $\widehat{J}(z ; \lambda ; \xi)$ as its asymptotic expansion on $\mathbb{S}_{\xi}^{ \pm}$or any of its open subsector. For the moment, we denote this solution by $J^{ \pm}(z ; \lambda ; \xi)$. On the other hand, because $\mathbb{S}_{\xi}$ has central angle $\frac{2}{n} \pi<\pi$, there exists a solution $J(z ; \lambda ; \xi)$ with asymptotic $\widehat{J}(z ; \lambda ; \xi)$ on a given open subsector $\mathbb{S} \subset \mathbb{S}_{\xi}$ due to Lemma 11.16 Observe that at least one of $\mathbb{S} \cap \mathbb{S}_{\xi}^{+}$and $\mathbb{S} \cap \mathbb{S}_{\xi}^{-}$is a nonempty open sector, say $\mathbb{S} \cap \mathbb{S}_{\xi}^{+} \neq \varnothing$, then the uniqueness of $J(z ; \lambda ; \xi)$ follows from that of $J^{+}(z ; \lambda ; \xi)$ along with the principle of analytic continuation.

Q.E.D.

Proposition 11.18. Let $\varsigma \in\{+,-\}, \xi \in \mathbb{X}_{n}(\varsigma), \vartheta$ be a small positive constant, say $0<\vartheta<\frac{1}{2} \pi$, and define

$$
\mathbb{S}_{\xi}^{\prime}(\vartheta)=\left\{z:|\arg z-\arg (i \bar{\xi})|<\pi+\frac{\pi}{n}-\vartheta\right\} .
$$

Then $J(z ; \lambda ; \xi)$ is the unique solution of the Bessel equation of sign $\varsigma$ that has the asymptotic expansion $\widehat{J}(z ; \lambda ; \xi)$ on $\mathbb{S}_{\xi}^{\prime}(\vartheta)$. Moreover, $J^{(j)}(z ; \lambda ; \xi) \sim \widehat{J}^{(j)}(z ; \lambda ; \xi)$ on $\mathbb{S}_{\xi}^{\prime}(\vartheta)$ for any $j \geqslant 0$.

Proof. Following from Lemma 11.16 , there exists a solution of the Bessel equation of sign $\varsigma$ that has the asymptotic expansion $\widehat{J}(z ; \lambda ; \xi)$ on the open sector

$$
\mathbb{S}_{\bar{\xi}}^{ \pm}(\vartheta)=\left\{z: \frac{\pi}{n}-\vartheta< \pm(\arg z-\arg (i \bar{\xi}))<\pi+\frac{\pi}{n}-\vartheta\right\} .
$$

On the nonempty open sector $\mathbb{S}_{\xi} \cap \mathbb{S}_{\xi}^{ \pm}(\vartheta)$ this solution must be identical with $J(z ; \lambda ; \xi)$ by Lemma 11.17 (2) and hence is equal to $J(z ; \lambda ; \xi)$ on $\mathbb{S}_{\xi} \cup \mathbb{S}_{\xi}^{ \pm}(\vartheta)$ due to the principle of analytic continuation. Therefore, the region of validity of the asymptotic $J(z ; \lambda ; \xi) \sim$ $\widehat{J}(z ; \lambda ; \xi)$ may be widened from $\mathbb{S}_{\xi}$ onto $\mathbb{S}_{\xi}^{\prime}(\vartheta)=\mathbb{S}_{\xi} \cup \mathbb{S}_{\xi}^{+}(\vartheta) \cup \mathbb{S}_{\xi}^{-}(\vartheta)$. In the same way, Lemma 11.16 and 11.17 (2) also imply that $J^{(j)}(z ; \lambda ; \xi) \sim \widehat{J}^{(j)}(z ; \lambda ; \xi)$ on $\mathbb{S}_{\xi}^{\prime}(\vartheta)$. Q.E.D. 
Corollary 11.19. Let $\varsigma \in\{+,-\}$. All the $J(z ; \lambda ; \xi)$, with $\xi \in \mathbb{X}_{n}(\varsigma)$, form a fundamental set of solutions of the Bessel equation of sign $\varsigma$.

Remark 11.20. If $n=2$, by [Wat 3.7 (8), $3.71(18), 7.2(1,2), 7.23(1,2)]$ we have the following formula of $J(z ; \lambda,-\lambda ; \xi)$, with $\xi= \pm 1, \pm i$, and the corresponding sector on which its asymptotic expansion is valid

$$
\begin{gathered}
J(z ; \lambda,-\lambda ; 1)=\sqrt{\pi i} e^{\pi i \lambda} H_{2 \lambda}^{(1)}(2 z), \quad \mathbb{S}_{1}^{\prime}(\vartheta)=\{z:-\pi+\vartheta<\arg z<2 \pi-\vartheta\} ; \\
J(z ; \lambda,-\lambda ;-1)=\sqrt{-\pi i} e^{-\pi i \lambda} H_{2 \lambda}^{(2)}(2 z), \quad \mathbb{S}_{-1}^{\prime}(\vartheta)=\{z:-2 \pi+\vartheta<\arg z<\pi-\vartheta\} ; \\
J(z ; \lambda,-\lambda ; i)=\frac{2}{\sqrt{\pi}} K_{2 \lambda}(2 z), \quad \mathbb{S}_{i}^{\prime}(\vartheta)=\left\{z:|\arg z|<\frac{3}{2} \pi-\vartheta\right\} ; \\
J(z ; \lambda,-\lambda ;-i)=2 \sqrt{\pi} I_{2 \lambda}(2 z)-\frac{2 i}{\sqrt{\pi}} e^{2 \pi i \lambda} K_{2 \lambda}(2 z), \\
\mathbb{S}_{-i}^{\prime}(\vartheta)=\left\{z:-\frac{1}{2} \pi+\vartheta<\arg z<\frac{5}{2} \pi-\vartheta\right\} .
\end{gathered}
$$

Lemma 11.21. Let $\xi \in \mathbb{X}_{2 n}(+)$. We have

$$
J(z ; \lambda ; \xi)=( \pm \xi)^{\frac{n-1}{2}} J( \pm \xi z ; \lambda ; \pm 1)
$$

and $B_{m}(\lambda ; \xi)=( \pm \xi)^{-m} B_{m}(\lambda ; \pm 1)$.

Proof. By Lemma 11.1 $( \pm \xi)^{\frac{n-1}{2}} J( \pm \xi z ; \lambda ; \pm 1)$ is a solution of one of the two Bessel equations of index $\lambda$. In view of Proposition 11.14 and Lemma 11.17 (2), it possesses $\widehat{J}(z ; \lambda ; \xi)$ as its asymptotic expansion on $\mathbb{S}_{\xi}$ and hence must be identical with $J(z ; \lambda ; \xi)$.

Q.E.D.

Terminology 11.22. For $\xi \in \mathbb{X}_{2 n}(+), J(z ; \lambda ; \xi)$ is called a Bessel function of the second kind.

REMARK 11.23. The results in this section do not provide any information on the asymptotics near zero of Bessel functions of the second kind, and therefore their connections with Bessel functions of the first kind can not be clarified here. We shall nevertheless derive the connection formulae between the two kinds of Bessel functions later in $\$ 12$. appealing to the asymptotic expansion of the $H$-Bessel function $H^{ \pm}(z ; \lambda)$ on the half-plane $\mathbb{H}^{ \pm}$that we showed earlier in $\$ 9$

11.4. Error Analysis for Asymptotic Expansions. The error bound for the asymptotic expansion of $J(z ; \lambda ; \xi)$ with dependence on $\lambda$ is always desirable for potential applications in analytic number theory. However, the author does not find any general results on the error analysis for differential equations of order higher than two. We shall nevertheless combine and generalize the ideas from [CL, §5.4] and [Olv3, \$7.2] to obtain an almost optimal error estimate for the asymptotic expansion of the Bessel function $J(z ; \lambda ; \xi)$. Observe that both of their methods have drawbacks for generalizations. [Olv3 hardly uses the viewpoint from differential systems as only the second-order case is treated, whereas [CL \$5.4] is restricted to the positive real axis for more clarified expositions.

11.4.1. Preparations. We retain the notations from $\$ 11.3 .1$ For a positive integer $M$ denote by $P_{(M)}$ the polynomial in $z^{-1}$,

$$
P_{(M)}(z)=\sum_{m=0}^{M} z^{-m} P_{m},
$$


and by $\widehat{\Phi}_{(M)}$ the truncation of $\widehat{\Phi}$,

$$
\widehat{\Phi}_{(M)}(z)=P_{(M)}(z) z^{-\frac{n-1}{2}} e^{\Delta z} .
$$

By Lemma 11.13 , we have $\left|z^{-m} P_{m}\right| \ll_{m, n} \mathfrak{C}^{2 m}|z|^{-m}$, so $P_{(M)}^{-1}$ exists as an analytic function for $|z|>c_{1} \mathbb{E}^{2}$, where $c_{1}$ is some constant depending only on $M$ and $n$. Moreover,

$$
\left|P_{(M)}(z)\right|,\left|P_{(M)}^{-1}(z)\right|=O_{M, n}(1), \quad|z|>c_{1} \mathbb{C}^{2} .
$$

Let $A_{(M)}$ and $E_{(M)}$ be defined by

$$
A_{(M)}=\widehat{\Phi}_{(M)}^{\prime} \widehat{\Phi}_{(M)}^{-1}, \quad E_{(M)}=A-A_{(M)} .
$$

$A_{(M)}$ and $E_{(M)}$ are clearly analytic for $|z|>c_{1} \mathfrak{C}^{2}$. Since

$$
E_{(M)} P_{(M)}=A P_{(M)}-\left(P_{(M)}^{\prime}-\frac{n-1}{2} z^{-1} P_{(M)}+P_{(M)} \Delta\right),
$$

it follows from the construction of $\widehat{\Phi}$ in $\$ 11.3 .1$ that $E_{(M)} P_{(M)}$ is a polynomial in $z^{-1}$ of the form $\sum_{m=M+1}^{M+n} z^{-m} E_{m}$ so that

$$
\begin{aligned}
E_{M+1} & =P_{M+1}^{o} \Delta-\Delta P_{M+1}^{o}, \\
E_{m} & =\sum_{j=m-M}^{\min \{m, n\}} A_{j} P_{m-j}, \quad M+1<m \leqslant M+n .
\end{aligned}
$$

Therefore, in view of Lemma $11.13,\left|E_{M+1}\right| \ll_{M, n} \mathbb{C}^{2 M}$ and $\left|E_{m}\right| \ll_{m, n} \mathbb{E}^{m+M}$ for $M+1<$ $m \leqslant M+n$. It follows that $\left|E_{(M)}(z) P_{(M)}(z)\right| \ll_{M, n} \mathfrak{C}^{2 M} z^{-M-1}$ for $|z|>c_{1} \mathfrak{C}^{2}$, and this, combined with 11.22, yields

$$
\left|E_{(M)}(z)\right|=O_{M, n}\left(\mathfrak{C}^{2 M}|z|^{-M-1}\right) .
$$

By the definition of $A_{(M)}$, for $|z|>c_{1} \mathbb{E}^{2}, \widehat{\Phi}_{(M)}$ is a fundamental matrix of the system

$$
u^{\prime}=A_{(M)} u \text {. }
$$

We shall regard the differential system (11.8), that is,

$$
u^{\prime}=A u=A_{(M)} u+E_{(M)} u,
$$

as a nonhomogeneous system with (11.24) as the corresponding homogeneous system.

11.4.2. Construction of a Solution. Given $l \in\{1, \ldots, n\}$, let

$$
\hat{\varphi}_{(M), l}(z)=p_{(M), l}(z) z^{-\frac{n-1}{2}} e^{i n \xi l z}
$$

be the $l$-th column vector of the matrix $\widehat{\Phi}_{(M)}$, where $p_{(M), l}$ is the $l$-th column vector of $P_{(M)}$. Using a version of the variation-of-constants formula and the method of successive approximations, we shall construct a solution $\varphi_{(M), l}$ of 11.8 , for $z$ in some suitable domain, satisfying

$$
\left|\varphi_{(M), l}(z)\right|=O_{M, n}\left(|z|^{-\frac{n-1}{2}} e^{\Re \mathfrak{e}(i n \xi \xi z)}\right)
$$

and

$$
\left|\varphi_{(M), l}(z)-\hat{\varphi}_{(M), l}(z)\right|=O_{M, n}\left(\mathfrak{C}^{2 M}|z|^{-M-\frac{n-1}{2}} e^{\Re e(i n \xi l z)}\right),
$$

with the implied constant in (11.27) also depending on the chosen domain. 


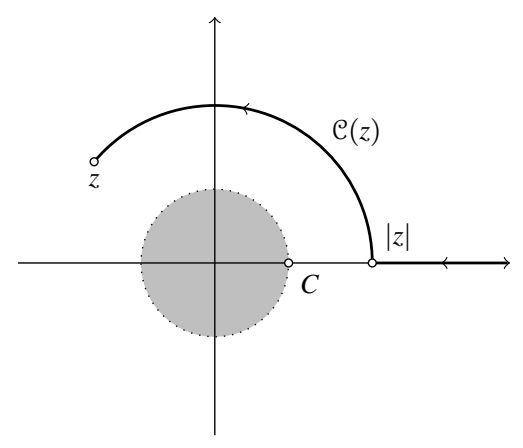

$|\arg z| \leqslant \pi$

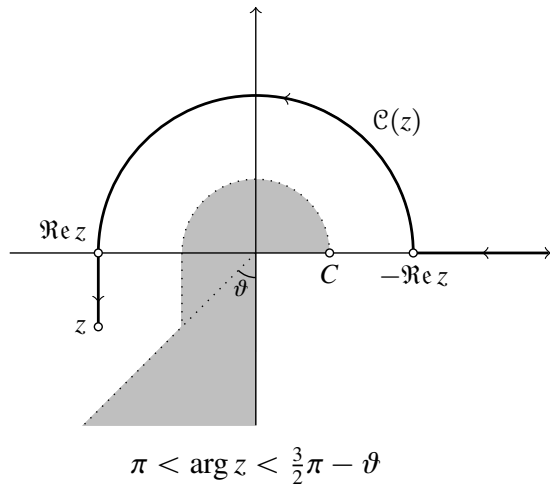

FIGURE 2. $\mathcal{C}(z) \subset \mathbb{D}(C ; \vartheta)$

Step 1. Constructing the domain and the contours for the integral equation. For $C \geqslant c_{1} \mathfrak{E}^{2}$ and $0<\vartheta<\frac{1}{2} \pi$, define the domain $\mathbb{D}(C ; \vartheta) \subset \mathbb{U}$ by

$$
\mathbb{D}(C ; \vartheta)=\{z:|\arg z| \leqslant \pi,|z|>C\} \cup\left\{z: \pi<|\arg z|<\frac{3}{2} \pi-\vartheta, \mathfrak{R e} z<-C\right\} .
$$

For $k \neq l$ let $\omega(l, k)=\arg \left(i \bar{\xi}_{l}-i \bar{\xi}_{k}\right)=\arg \left(i \bar{\xi}_{l}\right)+\arg \left(1-\xi_{k} \bar{\xi}_{l}\right)$, and define

$$
\mathbb{D}_{\xi_{l}}(C ; \vartheta)=\bigcap_{k \neq l} e^{i \omega(l, k)} \cdot \mathbb{D}(C ; \vartheta)
$$

With the observation that

$$
\left\{\arg \left(1-\xi_{k} \bar{\xi}_{l}\right): k \neq l\right\}=\left\{\left(\frac{1}{2}-\frac{a}{n}\right) \pi: a=1, \ldots, n-1\right\},
$$

it is straightforward to show that $\mathbb{D}_{\xi_{l}}(C ; \vartheta)=i \bar{\xi}_{l} \mathbb{D}^{\prime}(C ; \vartheta)$, where $\mathbb{D}^{\prime}(C ; \vartheta)$ is defined to be the union of the sector

$$
\left\{z:|\arg z| \leqslant \frac{\pi}{2}+\frac{\pi}{n},|z|>C\right\}
$$

and the following two domains

$$
\begin{aligned}
& \left\{z: \frac{\pi}{2}+\frac{\pi}{n}<\arg z<\pi+\frac{\pi}{n}-\vartheta, \mathfrak{I m}\left(e^{-\frac{1}{n} \pi i} z\right)>C\right\}, \\
& \left\{z:-\pi-\frac{\pi}{n}+\vartheta<\arg z<-\frac{\pi}{2}-\frac{\pi}{n}, \mathfrak{I m}\left(e^{\frac{1}{n} \pi i} z\right)<-C\right\} .
\end{aligned}
$$

For $z \in \mathbb{D}(C ; \vartheta)$ we define a contour $\mathcal{C}(z) \subset \mathbb{D}(C ; \vartheta)$ that starts from $\infty$ and ends at $z$; see Figure 2. For $z \in \mathbb{D}(C ; \vartheta)$ with $|\arg z| \leqslant \pi$, the contour $\mathcal{C}(z)$ consists of the part of the positive axis where the magnitude exceeds $|z|$ and an arc of the circle centered at the origin of radius $|z|$, with angle not exceeding $\pi$ and endpoint $z$. For $z \in \mathbb{D}(C ; \vartheta)$ with $\pi<|\arg z|<\frac{3}{2} \pi-\vartheta$, the definition of the contour $\mathcal{C}(z)$ is modified so that the circular arc has radius $-\mathfrak{R e} z$ instead of $|z|$ and ends at $\Re \mathfrak{e} z$ on the negative real axis, and that $\mathcal{C}(z)$ also consists of a vertical line segment joining $\mathfrak{R e} z$ and $z$. The most crucial property that $\mathcal{C}(z)$ satisfies is the nonincreasing of $\Re \mathrm{e} \zeta$ along $\mathrm{C}(z)$.

We also define a contour $\mathcal{C}^{\prime}(z)$ for $z \in \mathbb{D}^{\prime}(C ; \vartheta)$ of a similar shape as $\mathcal{C}(z)$ illustrated in Figure 3 


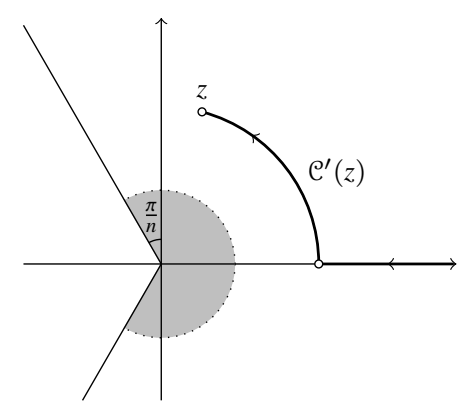

$|\arg z| \leqslant \frac{1}{2} \pi+\frac{1}{n} \pi$

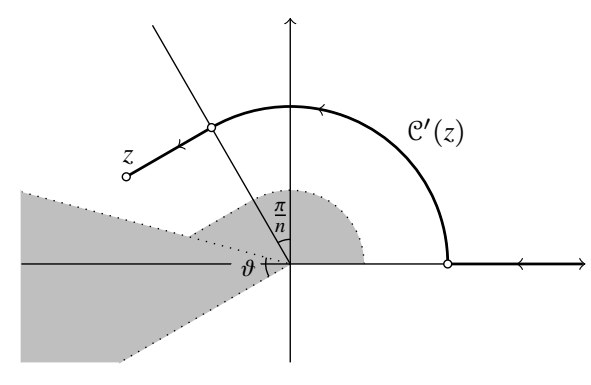

$\frac{1}{2} \pi+\frac{1}{n} \pi<\arg z<\pi+\frac{1}{n} \pi-\vartheta$

FIGURE 3. $\mathcal{C}^{\prime}(z) \subset \mathbb{D}^{\prime}(C ; \vartheta)$

Step 2. Solving the integral equation via successive approximations. We first split $\widehat{\Phi}_{(M)}^{-1}$ into $n$ parts

$$
\widehat{\Phi}_{(M)}^{-1}=\sum_{k=1}^{n} \Psi_{(M)}^{(k)},
$$

where the $j$-th row of $\Psi_{(M)}^{(k)}$ is identical with the $k$-th row of $\widehat{\Phi}_{(M)}^{-1}$, or identically zero, according as $j=k$ or not.

The integral equation to be considered is the following

$$
u(z)=\widehat{\varphi}_{(M), l}(z)+\sum_{k \neq l} \int_{\infty e^{i \omega(l, k)}}^{z} K_{k}(z, \zeta) u(\zeta) d \zeta+\int_{\infty i \bar{\xi}_{l}}^{z} K_{l}(z, \zeta) u(\zeta) d \zeta
$$

where

$$
K_{k}(z, \zeta)=\widehat{\Phi}_{(M)}(z) \Psi_{(M)}^{(k)}(\zeta) E_{(M)}(\zeta), \quad z, \zeta \in \mathbb{D}_{\xi_{l}}(C ; \vartheta), k=1, \ldots, n,
$$

the integral in the sum is integrated on the contour $e^{i \omega(l, k)} \mathcal{C}\left(e^{-i \omega(l, k)} z\right)$, whereas the last integral is on the contour $i \bar{\xi}_{l} \mathrm{e}^{\prime}\left(-i \xi_{l} z\right)$. Clearly, all these contours lie in $\mathbb{D}_{\xi_{l}}(C ; \vartheta)$. Most importantly, we note that $\mathfrak{R e}\left(\left(i \xi_{l}-i \xi_{k}\right) \zeta\right)$ is a negative multiple of $\mathfrak{R e}\left(e^{-i \omega(l, k)} \zeta\right)$ and hence is nondecreasing along the contour $e^{i \omega(l, k)} \mathfrak{C}\left(e^{-i \omega(l, k)} z\right)$.

By direct verification, it follows that if $u(z)=\varphi(z)$ satisfies 11.28, with the integrals convergent, then $\varphi$ satisfies 11.25.

In order to solve (11.28), define the successive approximations

$$
\begin{gathered}
\varphi^{0}(z) \equiv 0, \\
\varphi^{\alpha+1}(z)=\widehat{\varphi}_{(M), l}(z)+\sum_{k \neq l} \int_{\infty e^{i \omega(l, k)}}^{z} K_{k}(z, \zeta) \varphi^{\alpha}(\zeta) d \zeta+\int_{\infty i \bar{\xi}_{l}}^{z} K_{l}(z, \zeta) \varphi^{\alpha}(\zeta) d \zeta .
\end{gathered}
$$

The $(j, r)$-th entry of the matrix $\widehat{\Phi}_{(M)}(z) \Psi_{(M)}^{(k)}(\zeta)$ is given by

$$
\left(\widehat{\Phi}_{(M)}(z) \Psi_{(M)}^{(k)}(\zeta)\right)_{j r}=\left(P_{(M)}(z)\right)_{j k}\left(P_{(M)}^{-1}(\zeta)\right)_{k r}\left(\frac{z}{\zeta}\right)^{-\frac{n-1}{2}} e^{i n \xi_{k}(z-\zeta)} .
$$

It follows from 11.22 11.23 that

$$
\left|K_{k}(z, \zeta)\right| \leqslant c_{2} \mathfrak{C}^{2 M}|z|^{-\frac{n-1}{2}}|\zeta|^{-M-1+\frac{n-1}{2}} e^{\Re \mathrm{e}\left(i n \xi_{k}(z-\zeta)\right)},
$$


for some constant $c_{2}$ depending only on $M$ and $n$. Furthermore, we may appropriately choose $c_{2}$ such that

$$
\int_{\infty i \bar{\xi}_{l}}^{z}|\zeta|^{-M-1}|d \zeta|, \int_{\infty e^{i \omega(l, k)}}^{z}|\zeta|^{-M-1}|d \zeta| \leqslant c_{2} C^{-M}, \quad k \neq l .
$$

According to $11.29, \varphi^{1}(z)=\hat{\varphi}_{(M), l}(z)=p_{(M), l}(z) z^{-\frac{n-1}{2}} e^{i n \xi l z}$, so

$$
\left|\varphi^{1}(z)-\varphi^{0}(z)\right|=\left|\varphi^{1}(z)\right| \leqslant c_{2}|z|^{-\frac{n-1}{2}} e^{\Re\left(i n \xi_{l} z\right)}, \quad z \in \mathbb{D}_{\xi_{l}}(C ; \vartheta) .
$$

We shall show by induction that for all $z \in \mathbb{D}_{\xi_{l}}(C ; \vartheta)$

$$
\left|\varphi^{\alpha}(z)-\varphi^{\alpha-1}(z)\right| \leqslant c_{2}\left(n c_{2}^{2} \mathfrak{C}^{-2 M} C^{-M}\right)^{\alpha-1}|z|^{-\frac{n-1}{2}} e^{\Re \mathrm{R}\left(i n \xi_{l} z\right)} .
$$

Let $z \in \mathbb{D}_{\xi_{l}}(C ; \vartheta)$. Assume that 11.32 holds. From 11.29 we have

$$
\left|\varphi^{\alpha+1}(z)-\varphi^{\alpha}(z)\right| \leqslant \sum_{k \neq l} R_{k}+R_{l}
$$

with

$$
\begin{aligned}
& R_{k}=\int_{\infty e^{i \omega(l, k)}}^{z}\left|K_{k}(z, \zeta)\right|\left|\varphi^{\alpha}(\zeta)-\varphi^{\alpha-1}(\zeta)\right||d \zeta|, \\
& R_{l}=\int_{\infty i \bar{\zeta}_{l}}^{z}\left|K_{l}(z, \zeta)\right|\left|\varphi^{\alpha}(\zeta)-\varphi^{\alpha-1}(\zeta)\right||d \zeta| .
\end{aligned}
$$

It follows from 11.30 11.32 that $R_{k}$ has bound

$$
c_{2}^{2} \mathfrak{C}^{2 M}\left(n c_{2}^{2} \mathfrak{C}^{2 M} C^{-M}\right)^{\alpha-1}|z|^{-\frac{n-1}{2}} e^{\Re \mathrm{Re}(i n \xi \xi z)} \int_{\infty e^{i \omega(l, k)}}^{z}|\zeta|^{-M-1} e^{\Re \mathfrak{e}\left(i n\left(\xi_{l}-\xi_{k}\right)(\zeta-z)\right)}|d \zeta| .
$$

Since $\mathfrak{R e}\left(\left(i \xi_{l}-i \xi_{k}\right) \zeta\right)$ is nondecreasing on the integral contour,

$$
R_{k} \leqslant c_{2}^{2} \mathfrak{C}^{2 M}\left(n c_{2}^{2} \mathfrak{C}^{2 M} C^{-M}\right)^{\alpha-1}|z|^{-\frac{n-1}{2}} e^{\Re \mathrm{Re}(i n \xi l z)} \int_{\infty e^{i \omega(l, k)}}^{z}|\zeta|^{-M-1}|d \zeta|,
$$

and (11.31) further yields

$$
R_{k} \leqslant c_{2} n^{\alpha-1}\left(c_{2}^{2} \mathfrak{C}^{2 M} C^{-M}\right)^{\alpha}|z|^{-\frac{n-1}{2}} e^{\Re \mathrm{e}(i n \xi \mid z)} .
$$

Similar arguments show that $R_{l}$ has the same bound as $R_{k}$. Thus 11.32) is true with $\alpha$ replaced by $\alpha+1$.

Set the constant $C=c \mathfrak{C}^{2}$ such that $c^{M} \geqslant 2 n c_{2}^{2}$. Then $n c_{2}^{2} \mathfrak{C}^{2 M} C^{-M} \leqslant \frac{1}{2}$, and therefore the series $\sum_{\alpha=1}^{\infty}\left(\varphi^{\alpha}(z)-\varphi^{\alpha-1}(z)\right)$ absolutely and compactly converges. The limit function $\varphi_{(M), l}(z)$ satisfies 11.26 for all $z \in \mathbb{D}_{\xi_{l}}(C ; \vartheta)$. More precisely,

$$
\left|\varphi_{(M), l}(z)\right| \leqslant 2 c_{2}|z|^{-\frac{n-1}{2}} e^{\Re e\left(i n \xi_{l} / z\right)}, \quad z \in \mathbb{D}_{\xi_{l}}(C ; \vartheta) .
$$

Using a standard argument for successive approximations, it follows that $\varphi_{(M), l}$ satisfies the integral equation (11.28) and hence the differential system 11.25).

The proof of the error bound $(11.27)$ is similar. Since $\varphi_{(M), l}(z)$ is a solution of the integral equation (11.28, we have

$$
\left|\varphi_{(M), l}(z)-\hat{\varphi}_{(M), l}(z)\right| \leqslant \sum_{k \neq l} S_{k}+S_{l}
$$

where

$$
S_{k}=\int_{\infty e^{i \omega(l, k)}}^{z}\left|K_{k}(z, \zeta)\right|\left|\varphi_{(M), l}(\zeta)\right||d \zeta|, \quad S_{l}=\int_{\infty i \bar{\xi}_{l}}^{z}\left|K_{l}(z, \zeta)\right|\left|\varphi_{(M), l}(\zeta)\right||d \zeta| .
$$


With the observation that $|\zeta| \geqslant \sin \vartheta \cdot|z|$ for $z \in \mathbb{D}_{\xi_{l}}(C ; \vartheta)$ and $\zeta$ on the integral contours given above, we may replace (11.31) by the following

$$
\int_{\infty i \bar{\xi}_{l}}^{z}|\zeta|^{-M-1}|d \zeta|, \int_{\infty e^{i \omega(l, k)}}^{z}|\zeta|^{-M-1}|d \zeta| \leqslant c_{2}|z|^{-M}, \quad k \neq l,
$$

with $c_{2}$ now also depending on $\vartheta$.

The bounds 11.30 11.33 of $K_{k}(z, \zeta)$ and $\varphi_{(M), l}(z)$ along with 11.34 yield

$$
\begin{aligned}
S_{k} & \leqslant 2 c_{2}^{2} \mathfrak{C}^{2 M}|z|^{-\frac{n-1}{2}} e^{\Re \mathrm{R}(i n \xi \xi z)} \int_{\infty e^{i \omega(l, k)}}^{z}|\zeta|^{-M-1} e^{\Re \mathrm{R}\left(i n\left(\xi_{l}-\xi_{k}\right)(\zeta-z)\right)}|d \zeta| \\
& \leqslant 2 c_{2}^{2} \mathfrak{E}^{2 M}|z|^{-\frac{n-1}{2}} e^{\Re \mathrm{R}(i n \xi \mid z)} \int_{\infty e^{i \omega(l, k)}}^{z}|\zeta|^{-M-1}|d \zeta| \\
& \leqslant 2 c_{2}^{3} \mathfrak{C}^{2 M}|z|^{-M-\frac{n-1}{2}} e^{\Re \mathrm{Re}(i n \xi \xi)} .
\end{aligned}
$$

Again, the second inequality follows from the fact that $\Re \mathfrak{e}\left(\left(i \xi_{l}-i \xi_{k}\right) \zeta\right)$ is nondecreasing on the integral contour. Similarly, $S_{l}$ has the same bound as $S_{k}$. Thus (11.27) is proven and can be made precise as below

$$
\left|\varphi_{(M), l}(z)-\hat{\varphi}_{(M), l}(z)\right| \leqslant 2 n c_{2}^{3} \mathbb{C}^{2 M}|z|^{-M-\frac{n-1}{2}} e^{\Re e\left(i n \xi_{l} z\right)}, \quad z \in \mathbb{D}_{\xi_{l}}(C ; \vartheta) .
$$

11.4.3. Conclusion. Restricting to the sector $\mathbb{S}_{\xi_{l}}^{ \pm} \cap\{z:|z|>C\} \subset \mathbb{D}_{\xi_{l}}(C ; \vartheta)$, with $\mathbb{S}_{\xi_{l}}^{+}$replaced by $\mathbb{S}_{\xi_{l}}$ if $n=2$, each $\varphi_{(M), l}$ has an asymptotic representation a multiple of $\hat{\varphi}_{k}$ for some $k$ according to Lemma 11.17 (1). Since $\mathfrak{R e}\left(i \xi_{l} z\right)<\mathfrak{R e}\left(i \xi_{j} z\right)$ for all $j \neq l$, the bound $(11.26)$ forces $k=l$. Therefore, for any positive integer $M, \varphi_{(M), l}$ is identical with the unique solution $\varphi_{l}$ of the differential system $\sqrt{11.8}$ with asymptotic expansion $\hat{\varphi}_{l}$ on $\mathbb{S}_{\xi_{l}}^{ \pm}$ (see Lemma 11.17). Replacing $\varphi_{(M), l}$ by $\varphi_{l}$ and absorbing the $M$-th term of $\hat{\varphi}_{(M), l}$ into the error bound, we may reformulate (11.35) as the following error bound for $\varphi_{l}$

$$
\left|\varphi_{l}(z)-\hat{\varphi}_{(M-1), l}(z)\right|=O_{M, \vartheta, n}\left(\mathfrak{C}^{2 M}|z|^{-M-\frac{n-1}{2}} e^{\Re \mathrm{e}\left(i n \xi_{\xi} z\right)}\right), \quad z \in \mathbb{D}_{\xi_{l}}(C ; \vartheta) .
$$

Moreover, in view of the definition of the sector $\mathbb{S}_{\xi_{l}}^{\prime}(\vartheta)$ given in 11.21 , we have

$$
\mathbb{S}_{\xi_{l}}^{\prime}(\vartheta) \cap\left\{z:|z|>\frac{C}{\sin \vartheta}\right\} \subset \mathbb{D}_{\xi_{l}}(C ; \vartheta) .
$$

Thus the following theorem is finally established by (11.36) and (11.37).

Theorem 11.24. Let $\varsigma \in\{+,-\}, \xi \in \mathbb{X}_{n}(\varsigma), 0<\vartheta<\frac{1}{2} \pi, \mathbb{S}_{\xi}^{\prime}(\vartheta)$ be the sector defined as in (11.21), and $M$ be a positive integer. Then there exists a constant $c$, depending only on $M, \vartheta$ and $n$, such that

$$
J(z ; \lambda ; \xi)=e^{i n \xi z} z^{-\frac{n-1}{2}}\left(\sum_{m=0}^{M-1} B_{m}(\lambda ; \xi) z^{-m}+O_{M, \vartheta, n}\left(\mathfrak{C}^{2 M}|z|^{-M}\right)\right)
$$

for all $z \in \mathbb{S}_{\xi}^{\prime}(\vartheta)$ such that $|z|>c \mathfrak{C}^{2}$. Similar asymptotic is valid for all the derivatives of $J(z ; \lambda ; \xi)$, where the implied constant of the error estimate is allowed to depend on the order of the derivative.

Finally, we remark that, since $B_{m}(\lambda ; \xi) z^{-m}$ is of size $O_{m, n}\left(\mathfrak{C}^{2 m}|z|^{-m}\right)$, the error bound in 11.38 is optimal, given that $\vartheta$ is fixed. 


\section{Connections between Various Types of Bessel Functions}

Recall from $\$ 9.4 .1$ that the asymptotic expansion in Theorem 9.11 remains valid for the $H$-Bessel function $H^{ \pm}(z ; \lambda)$ on the half-plane $\mathbb{H}^{ \pm}=\{z: 0 \leqslant \pm \arg z \leqslant \pi\}$ (see (9.11)). With the observations that $H^{ \pm}(z ; \lambda)$ satisfies the Bessel equation of sign $( \pm)^{n}$, that the asymptotic expansions of $\sqrt{n}( \pm 2 \pi i)^{-\frac{n-1}{2}} H^{ \pm}(z ; \lambda)$ and $J(z ; \lambda ; \pm 1)$ have exactly the same form and the same leading term due to Theorem 9.11 and Proposition 11.14, and that $\mathbb{S}_{ \pm 1}=\left\{z:\left(\frac{1}{2}-\frac{1}{n}\right) \pi< \pm \arg z<\left(\frac{1}{2}+\frac{1}{n}\right) \pi\right\} \subset \mathbb{H}^{ \pm}$, Lemma 11.17 (2) implies the following theorem.

THEOREM 12.1. We have

$$
H^{ \pm}(z ; \lambda)=n^{-\frac{1}{2}}( \pm 2 \pi i)^{\frac{n-1}{2}} J(z ; \lambda ; \pm 1),
$$

and $B_{m}(\lambda ; \pm 1)=( \pm i)^{-m} B_{m}(\lambda)$.

REMARK 12.2. The reader should observe that $\mathbb{S}_{ \pm 1} \cap \mathbb{R}_{+}=\varnothing$, so Theorem 12.1 can not be obtained by the asymptotic expansion of $H^{ \pm}(x ; \lambda)$ on $\mathbb{R}_{+}$derived from Stirling's asymptotic formula in Appendix B (see Remark B.1).

REMARK 12.3. $B_{m}(\lambda ; \pm 1)$ can only be obtained from certain recurrence relations in $\$ 11.3 .1$ from the differential equation aspect. On the other hand, using the stationary phase method, 9.9 in $\$ 9.3$ yields an explicit formula of $B_{m}(\lambda)$. Thus Theorem 12.1 indicates that the recurrence relations for $B_{m}(\lambda ; \pm 1)$ are actually solvable!

As consequences of Theorem 12.1, we can establish the connections between various Bessel functions, that is, $J(z ; \boldsymbol{\varsigma}, \lambda), J_{l}(z ; \varsigma, \lambda)$ and $J(z ; \lambda ; \xi)$. Recall that $J(z ; \boldsymbol{\varsigma}, \lambda)$ has already been expressed in terms of $J_{l}(z ; \varsigma, \lambda)$ in Lemma 11.7

12.1. Relations between $J(z ; \boldsymbol{\varsigma}, \lambda)$ and $J(z ; \lambda ; \xi) . J(z ; \boldsymbol{\varsigma}, \lambda)$ is equal to a multiple of $H^{ \pm}\left(e^{ \pm \pi i \frac{{ }^{n} \frac{\eta}{n}(s)}{n}} z ; \lambda\right)$ due to Lemma 11.10 whereas $J(z ; \lambda ; \xi)$ is a multiple of $J( \pm \xi z ; \lambda ; \pm 1)$ in view of Lemma 11.21 . Furthermore, the equality, up to constant, between $H^{ \pm}(z ; \lambda)$ and $J(z ; \lambda ; \pm 1)$ has just been established in Theorem 12.1 . We then arrive at the following corollary.

Corollary 12.4. Let $L_{ \pm}(\boldsymbol{s})=\left\{l: \varsigma_{l}= \pm\right\}$ and $n_{ \pm}(\boldsymbol{s})=\left|L_{ \pm}(\boldsymbol{s})\right|$ be as in Definition 11.6. Let $c(\boldsymbol{s}, \lambda)=e\left(\mp \frac{n-1}{8} \pm \frac{(n-1) n_{ \pm}(\boldsymbol{s})}{4 n} \mp \frac{1}{2} \sum_{l \in L_{ \pm}(\boldsymbol{s})} \lambda_{l}\right)$ and $\xi(\boldsymbol{s})=\mp e^{\mp \pi i \frac{{ }^{n_{ \pm}}(\boldsymbol{s})}{n}}$. Then

$$
J(z ; \boldsymbol{\varsigma}, \lambda)=\frac{(2 \pi)^{\frac{n-1}{2}} c(\boldsymbol{\varsigma}, \lambda)}{\sqrt{n}} J(z ; \lambda ; \xi(\boldsymbol{\varsigma})) .
$$

Here, it is understood that $\arg \xi(\boldsymbol{\varsigma})=\frac{n_{-}(\boldsymbol{\varsigma})}{n} \pi=\pi-\frac{n_{+}(\boldsymbol{\varsigma})}{n} \pi$.

Corollary 12.4 shows that $J(z ; \boldsymbol{\varsigma}, \lambda)$ should really be categorized in the class of Bessel functions of the second kind. Moreover, the asymptotic behaviours of the Bessel functions $J(z ; \boldsymbol{\varsigma}, \lambda)$ are classified by their signatures $\left(n_{+}(\boldsymbol{\varsigma}), n_{-}(\boldsymbol{\varsigma})\right)$. Therefore, $J(z ; \boldsymbol{\varsigma}, \lambda)$ is uniquely determined by its signature up to a constant multiple.

12.2. Relations Connecting the Two Kinds of Bessel Functions. From Lemma 11.21 and Theorem 12.1 , one sees that $J(z ; \lambda ; \xi)$ is a constant multiple of $H^{+}(\xi z ; \lambda)$. On the other hand, $H^{+}(z ; \lambda)$ can be expressed in terms of Bessel functions of the first kind in view of Lemma 11.7 . Finally, using Lemma 11.3 the following corollary is readily established. 
Corollary 12.5. Let $\varsigma \in\{+,-\}$. If $\xi \in \mathbb{X}_{n}(\varsigma)$, then

$$
J(z ; \lambda ; \xi)=\sqrt{n}\left(-\frac{\pi i \xi}{2}\right)^{\frac{n-1}{2}} \sum_{l=1}^{n}(i \bar{\xi})^{n \lambda_{l}} S_{l}(\lambda) J_{l}(z ; \varsigma, \lambda),
$$

with $S_{l}(\lambda)=1 / \prod_{k \neq l} \sin \left(\pi\left(\lambda_{l}-\lambda_{k}\right)\right)$. According to our convention, we have $(-i \xi)^{\frac{n-1}{2}}=$ $e^{\frac{n-1}{2}\left(-\frac{1}{2} \pi i+i \arg \xi\right)}$ and $(i \bar{\xi})^{n \lambda_{l}}=e^{\frac{1}{2} \pi i n \lambda_{l}-i n \lambda_{l} \arg \xi}$. When $\lambda$ is not generic, the right hand side should be replaced by its limit.

We now fix an integer $a$ and let $\xi_{j}=e^{\pi i \frac{2 j+a-2}{n}} \in \mathbb{X}_{n}\left((-)^{a}\right)$, with $j=1, \ldots, n$. It follows from Corollary 12.5 that

$$
X(z ; \lambda)=\sqrt{n}\left(\frac{\pi}{2}\right)^{\frac{n-1}{2}} e^{-\frac{1}{4} \pi i(n-1)} \cdot D V(\lambda) S(\lambda) E(\lambda) Y(z ; \lambda),
$$

with

$$
\begin{aligned}
& X(z ; \lambda)=\left(J\left(z ; \lambda ; \xi_{j}\right)\right)_{j=1}^{n}, \quad Y(z ; \lambda)=\left(J_{l}\left(z ;(-)^{a}, \lambda\right)\right)_{l=1}^{n}, \\
& D=\operatorname{diag}\left(\xi_{j}^{\frac{n-1}{2}}\right)_{j=1}^{n}, \quad E(\lambda)=\operatorname{diag}\left(e^{\pi i\left(\frac{1}{2} n-a\right) \lambda_{l}}\right)_{l=1}^{n}, \quad S(\lambda)=\operatorname{diag}\left(S_{l}(\lambda)\right)_{l=1}^{n}, \\
& V(\lambda)=\left(e^{-2 \pi i(j-1) \lambda_{l}}\right)_{j, l=1}^{n} .
\end{aligned}
$$

Observe that $V(\lambda)$ is a Vandermonde matrix.

Lemma 12.6. For an $n$-tuple $\boldsymbol{x}=\left(x_{1}, \ldots, x_{n}\right) \in \mathbb{C}^{n}$ we define the Vandermonde matrix $V=\left(x_{l}^{j-1}\right)_{j, l=1}^{n}$. For $d=0,1, \ldots, n-1$ and $m=1, \ldots, n$, let $\sigma_{m, d}$ denote the elementary symmetric polynomial in $x_{1}, \ldots, \widehat{x_{m}}, \ldots, x_{n}$ of degree $d$, and let $\tau_{m}=\prod_{k \neq m}\left(x_{m}-x_{k}\right)$. If $\boldsymbol{x}$ is generic in the sense that all the components of $\boldsymbol{x}$ are distinct, then $V$ is invertible, and furthermore, the inverse of $V$ is $\left((-)^{n-j} \sigma_{m, n-j} \tau_{m}^{-1}\right)_{m, j=1}^{n}$.

Proof of Lemma 12.6 It is a well-known fact that $V$ is invertible whenever $\boldsymbol{x}$ is generic. If one denotes by $w_{m, j}$ the $(m, j)$-th entry of $V^{-1}$, then

$$
\sum_{j=1}^{n} w_{m, j} x_{l}^{j-1}=\delta_{m, l}
$$

The Lagrange interpolation formula implies the following identity of polynomials

$$
\sum_{j=1}^{n} w_{m, j} x^{j-1}=\prod_{k \neq m} \frac{x-x_{k}}{x_{m}-x_{k}}
$$

Identifying the coefficients of $x^{j-1}$ on both sides yields the desired formula of $w_{m, j}$. Q.E.D.

Corollary 12.7. Let a be a given integer. For $j=1, \ldots, n$ define $\xi_{j}=e^{\pi i \frac{2 j+a-2}{n}}$. For $d=0,1, \ldots, n-1$ and $l=1, \ldots, n$, let $\sigma_{l, d}(\lambda)$ denote the elementary symmetric polynomial in $e^{-2 \pi i \lambda_{1}}, \ldots, \widehat{e^{-2 \pi i \lambda_{l}}}, \ldots, e^{-2 \pi i \lambda_{n}}$ of degree $d$. Then

$$
J_{l}\left(z ;(-)^{a}, \lambda\right)=\frac{e^{\frac{3}{4} \pi i(n-1)}}{\sqrt{n}(2 \pi)^{\frac{n-1}{2}}} e^{\pi i\left(\frac{1}{2} n+a-2\right) \lambda_{l}} \sum_{j=1}^{n}(-)^{n-j} \xi_{j}^{-\frac{n-1}{2}} \sigma_{l, n-j}(\lambda) J\left(z ; \lambda ; \xi_{j}\right) .
$$

Proof. Choosing $x_{l}=e^{-2 \pi i \lambda_{l}}$ in Lemma 12.6 , one sees that if $\lambda$ is generic then the matrix $V(\lambda)$ is invertible and its inverse is given by

$$
\left((-2 i)^{1-n} \cdot(-)^{n-j} \sigma_{l, n-j}(\lambda) e^{\pi i(n-2) \lambda_{l}} S_{l}(\lambda)\right)_{l, j=1}^{n} .
$$


Some straightforward calculations then complete the proof.

Q.E.D.

Remark 12.8. In view of Proposition 4.4 Remark 11.4 and 11.20 , when $n=2$, Corollary 12.5 corresponds to the connection formulae (Wat 3.61(5, 6), 3.7 (6)]),

$$
\begin{array}{ll}
H_{v}^{(1)}(z)=\frac{J_{-v}(z)-e^{-\pi i v} J_{v}(z)}{i \sin (\pi v)}, & H_{v}^{(2)}(z)=\frac{e^{\pi i v} J_{v}(z)-J_{-v}(z)}{i \sin (\pi v)} \\
K_{v}(z)=\frac{\pi\left(I_{-v}(z)-I_{v}(z)\right)}{2 \sin (\pi v)}, & \pi I_{v}(z)-i e^{\pi i v} K_{v}(z)=\frac{\pi i\left(e^{-\pi i v} I_{v}(z)-e^{\pi i v} I_{-v}(z)\right)}{2 \sin (\pi v)},
\end{array}
$$

whereas Corollary 12.7, with $a=0$ or 1 , amounts to the formulae (see Wat 3.61 $(1,2)$, $3.7(6)])$

$$
\begin{array}{ll}
J_{v}(z)=\frac{H_{v}^{(1)}(z)+H_{v}^{(2)}(z)}{2}, & J_{-v}(z)=\frac{e^{\pi i v} H_{v}^{(1)}(z)+e^{-\pi i v} H_{v}^{(2)}(z)}{2}, \\
I_{v}(z)=\frac{i e^{\pi i v} K_{v}(z)+\left(\pi I_{v}(z)-i e^{\pi i v} K_{v}(z)\right)}{\pi}, I_{-v}(z) & =\frac{i e^{-\pi i v} K_{v}(z)+\left(\pi I_{v}(z)-i e^{\pi i v} K_{v}(z)\right)}{\pi} .
\end{array}
$$

\section{3. $H$-Bessel Functions and $K$-Bessel Functions, II}

In this concluding section, we apply Theorem 11.24 to improve the results in $\$ 9$ on the asymptotics of Bessel functions $J(x ; \boldsymbol{\varsigma}, \lambda)$ for $x \gg \mathbb{C}^{2}$.

13.1. Asymptotic Expansions of $H$-Bessel Functions. The following proposition is a direct consequence of Theorem 11.24 and 12.1 .

Proposition 13.1. Let $0<\vartheta<\frac{1}{2} \pi$.

(1). Let $M$ be a positive integer. We have

$$
\begin{aligned}
H^{ \pm}(z ; \lambda)=n^{-\frac{1}{2}} & ( \pm 2 \pi i)^{\frac{n-1}{2}} e^{ \pm i n z} z^{-\frac{n-1}{2}} \\
& \left(\sum_{m=0}^{M-1}( \pm i)^{-m} B_{m}(\lambda) z^{-m}+O_{M, \vartheta, n}\left(\mathfrak{C}^{2 M}|z|^{-M}\right)\right),
\end{aligned}
$$

for all $z \in \mathbb{S}_{ \pm 1}^{\prime}(\vartheta)$ such that $|z| \gg_{M, \vartheta, n} \mathbb{C}^{2}$.

(2). Define $W^{ \pm}(z ; \lambda)=\sqrt{n}( \pm 2 \pi i)^{-\frac{n-1}{2}} e^{\mp i n z} H^{ \pm}(z ; \lambda)$. Let $M-1 \geqslant j \geqslant 0$. We have

$$
W^{ \pm,(j)}(z ; \lambda)=z^{-\frac{n-1}{2}}\left(\sum_{m=j}^{M-1}( \pm i)^{j-m} B_{m, j}(\lambda) z^{-m}+O_{M, \vartheta, j, n}\left(\mathfrak{C}^{2 M-2 j}|z|^{-M}\right)\right),
$$

for all $z \in \mathbb{S}_{ \pm 1}^{\prime}(\vartheta)$ such that $|z| \gg_{M, \vartheta, n} \mathfrak{C}^{2}$.

Observe that

$$
\begin{aligned}
& \mathbb{H}^{ \pm}=\{z \in \mathbb{C}: 0 \leqslant \pm \arg z \leqslant \pi\} \\
& \subset \mathbb{S}_{ \pm 1}^{\prime}(\vartheta)=\left\{z \in \mathbb{U}:-\left(\frac{1}{2}-\frac{1}{n}\right) \pi-\vartheta< \pm \arg z<\left(\frac{3}{2}+\frac{1}{n}\right) \pi+\vartheta\right\} .
\end{aligned}
$$

Fixing $\vartheta$ and restricting to the domain $\left\{z \in \mathbb{H}^{ \pm}:|z| \gg_{M, n} \mathfrak{C}^{2}\right\}$, Proposition 13.1 improves Theorem 9.11 . 
13.2. Exponential Decay of $K$-Bessel Functions. Now suppose that $J(z ; \boldsymbol{\varsigma}, \lambda)$ is a $K$-Bessel function so that $0<n_{ \pm}(\boldsymbol{S})<n$. Since $\mathbb{R}_{+} \subset \mathbb{S}_{\xi(\boldsymbol{S})}^{\prime}(\vartheta)$, Corollary 12.4 and Theorem 11.24 imply that $J(x ; \boldsymbol{s}, \lambda)$, as well as all its derivatives, is not only a Schwartz function at infinity, which was shown in Theorem 9.6 but also a function of exponential decay on $\mathbb{R}_{+}$.

Proposition 13.2. If $J(x ; \boldsymbol{\varsigma}, \lambda)$ is a K-Bessel function, then for all $x \gg_{n} \mathfrak{C}^{2}$

$$
J^{(j)}(x ; \boldsymbol{s}, \lambda) \ll_{j, n} x^{-\frac{n-1}{2}} e^{-\pi \Im \mathfrak{m} \Lambda(\boldsymbol{s}, \lambda)-n I(\boldsymbol{s}) x},
$$

where $\Lambda(\boldsymbol{s}, \lambda)=\mp \sum_{l \in L_{ \pm}(\boldsymbol{s})} \lambda_{l}$ and $I(\boldsymbol{s})=\mathfrak{I m} \xi(\boldsymbol{s})=\sin \left(\frac{n_{ \pm}(\boldsymbol{s})}{n} \pi\right)>0$. In particular, we have

$$
J^{(j)}(x ; \boldsymbol{\varsigma}, \lambda) \ll_{j, n} x^{-\frac{n-1}{2}} e^{\pi \widetilde{\mathcal{I}}-n \sin \left(\frac{1}{n} \pi\right) x},
$$

for all $K$-Bessel functions $J(x ; \boldsymbol{\varsigma}, \lambda)$ with given $\lambda$, where $\mathfrak{I}=\max \left\{\left|\mathfrak{I m} \lambda_{l}\right|\right\}$.

\section{B. An Alternative Approach to Asymptotic Expansions}

When $n=3$, the application of Stirling's asymptotic formula in deriving the asymptotic expansion of a Hankel transform was first found in [Mil, \$4]. The asymptotic was later formulated more explicitly in [Li2, Lemma 6.1], where the author attributed the arguments in her proof to [Ivi]. Furthermore, using similar ideas as in [Mil], [B]0] simplified

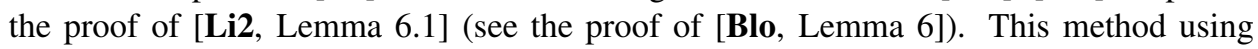
Stirling's asymptotic formula is however the only known approach so far in the literature.

Closely following [B]0], we shall prove the asymptotic expansions of $H$-Bessel functions $H^{ \pm}(x ; \lambda)$ of any rank $n$ by means of Stirling's asymptotic formula.

From 1.1, $1.2,3.6$ we have

$$
H^{ \pm}(x ; \lambda)=\frac{1}{2 \pi i} \int_{\mathcal{C}}\left(\prod_{l=1}^{n} \Gamma\left(s-\lambda_{l}\right)\right) e\left( \pm \frac{n s}{4}\right) x^{-n s} d s .
$$

In view of the condition $\sum_{l=1}^{n} \lambda_{l}=0$, Stirling's asymptotic formula yields

$$
\prod_{l=1}^{n} \Gamma\left(s-\lambda_{l}\right)=n^{-n s} \Gamma\left(n s-\frac{n-1}{2}\right) \exp \left(\sum_{m=0}^{M} C_{m}(\lambda) s^{-m}\right)\left(1+R_{M+1}(s)\right)
$$

for some constants $C_{m}(\lambda)$ and remainder term $R_{M+1}(s)=O_{\lambda, M, n}\left(|s|^{-M-1}\right)$. Using the Taylor expansion for the exponential function and some straightforward algebraic manipulations, the right hand side can be written as

$$
n^{-n s} \sum_{m=0}^{M} \widetilde{C}_{m}(\lambda) \Gamma\left(n s-\frac{n-1}{2}-m\right)\left(1+\widetilde{R}_{M+1, m}(s)\right)
$$

for certain constants $\widetilde{C}_{m}(\lambda)$ and similar functions $\widetilde{R}_{M+1, m}(s)=O_{\lambda, M, n}\left(|s|^{-M-1}\right)$. Suitably choosing the contour $\mathrm{C}$, it follows from 4.2 that

$$
\begin{aligned}
& \frac{1}{2 \pi i} \int_{\mathcal{C}} \Gamma\left(n s-\frac{n-1}{2}-m\right) e\left( \pm \frac{n s}{4}\right)(n x)^{-n s} d s \\
= & \frac{e\left( \pm\left(\frac{n-1}{8}+\frac{1}{4} m\right)\right)}{n(n x)^{\frac{n-1}{2}+m}} \cdot \frac{1}{2 \pi i} \int_{n \mathcal{C}-\frac{n-1}{2}-m} \Gamma(s) e\left( \pm \frac{s}{4}\right)(n x)^{-s} d s \\
= & \frac{( \pm i)^{\frac{n-1}{2}+m}}{n^{\frac{n+1}{2}}+m} \cdot \frac{e^{ \pm i n x}}{x^{\frac{n-1}{2}+m}} .
\end{aligned}
$$


As for the error estimate, let us assume $x \geqslant 1$. Insert the part containing $\widetilde{R}_{M+1, m}(s)$ into B.1) and shift the contour to the vertical line of real part $\frac{1}{n}\left(M-\frac{1}{2}\right)+\frac{1}{2}$. By Stirling's asymptotic formula, the integral remains absolutely convergent and is of size $O_{\lambda, M, n}\left(x^{-M-\frac{n-1}{2}}\right)$. Absorbing the last main term into the error, we arrive at the following asymptotic expansion

$$
H^{ \pm}(x ; \lambda)=e^{ \pm i n x} x^{-\frac{n-1}{2}}\left(\sum_{m=0}^{M-1} C_{m}^{ \pm}(\lambda) x^{-m}+O_{\lambda, M, n}\left(x^{-M}\right)\right), \quad x \geqslant 1,
$$

where $C_{m}^{ \pm}(\lambda)$ is some constant depending on $\lambda$.

Remark B.1. For the analytic continuation $H^{ \pm}(z ; \lambda)$, we have the Barnes type integral representation as in $\$ 3.1 .2$. This however does not yield an asymptotic expansion of $H^{ \pm}(z ; \lambda)$ by the above method. The obvious issue is with the error estimate, as $\left|z^{-n s}\right|$ is unbounded on the integral contour if $|z| \rightarrow \infty$.

Finally, we make some comparisons between the three asymptotic expansions $(B .2)$, 9.11) and (13.1) obtained from

- Stirling's asymptotic formula,

- the method of stationary phase,

- the asymptotic method of ordinary differential equations.

Recall that $\mathfrak{C}=\max \left\{\left|\lambda_{l}\right|\right\}+1, \mathfrak{R}=\max \left\{\left|\mathfrak{R e} \lambda_{l}\right|\right\}$. Firstly, the admissible domains of these asymptotic expansions are

$$
\begin{aligned}
& \left\{x \in \mathbb{R}_{+}: x \geqslant 1\right\}, \\
& \{z \in \mathbb{C}:|z| \geqslant \mathbb{C}, 0 \leqslant \pm \arg z \leqslant \pi\}, \\
& \left\{z \in \mathbb{U}:|z| \gg_{M, \vartheta, n} \mathbb{C}^{2},-\left(\frac{1}{2}-\frac{1}{n}\right) \pi-\vartheta< \pm \arg z<\left(\frac{3}{2}+\frac{1}{n}\right) \pi+\vartheta\right\},
\end{aligned}
$$

respectively. The range of argument is extending while that of modulus is reducing. Secondly, the error estimates are

$$
O_{\lambda, M, n}\left(x^{-M-\frac{n-1}{2}}\right), O_{\Re, M, n}\left(\mathfrak{C}^{2 M}|z|^{-M}\right), O_{M, \vartheta, n}\left(\mathfrak{C}^{2 M}|z|^{-M-\frac{n-1}{2}}\right),
$$

respectively. Thus, in the error estimate, the dependence of the implied constant on $\lambda$ is improving in all aspects. 



\section{CHAPTER 3}

\section{Bessel Kernels}

In this chapter, we shall return to the study of Bessel kernels, with emphasis on two connection formulae and the asymptotic expansion for the complex Bessel kernel $J_{(\mu, m)}(z)$.

\section{The Asymptotic of $J_{(\lambda, \delta)}(x)$}

According to $3.48, J_{(\lambda, \delta)}( \pm x)$ is a combination of $J\left(2 \pi x^{\frac{1}{n}} ; \boldsymbol{s}, \lambda\right)$, and hence its asymptotic follows immediately from Theorem 9.6 and 9.11 in $\$ 9$ as well as Proposition 13.1 and 13.2 in $\$ 13$. For convenience of reference, we record the asyptotic of $J_{(\lambda, \delta)}( \pm x)$ in the following theorem.

Theorem 14.1. Let $(\boldsymbol{\lambda}, \boldsymbol{\delta}) \in \mathbb{L}^{n-1} \times(\mathbb{Z} / 2 \mathbb{Z})^{n}$. Put $\mathfrak{C}(\boldsymbol{\lambda})=\max \left\{\left|\lambda_{l}\right|\right\}+1, \mathfrak{R}(\boldsymbol{\lambda})=$ $\max \left\{\left|\mathfrak{R e} \lambda_{l}\right|\right\}$ and $\mathfrak{I}(\boldsymbol{\lambda})=\max \left\{\left|\mathfrak{I m} \lambda_{l}\right|\right\}$. Let $M \geqslant 0$. Then, for $x>0$, we may write

$$
\begin{aligned}
& J_{(\lambda, \delta)}\left(x^{n}\right)=\sum_{ \pm} \frac{( \pm)^{|\delta|} e\left( \pm\left(n x+\frac{n-1}{8}\right)\right)}{n^{\frac{1}{2}} x^{\frac{n-1}{2}}} W_{\lambda}^{ \pm}(x)+E_{(\lambda, \delta)}^{+}(x), \\
& J_{(\lambda, \delta)}\left(-x^{n}\right)=E_{(\lambda, \delta)}^{-}(x),
\end{aligned}
$$

if $n$ is even, and

$$
J_{(\lambda, \delta)}\left( \pm x^{n}\right)=\frac{( \pm)^{|\delta|} e\left( \pm\left(n x+\frac{n-1}{8}\right)\right)}{n^{\frac{1}{2}} x^{\frac{n-1}{2}}} W_{\lambda}^{ \pm}(x)+E_{(\lambda, \delta)}^{ \pm}(x),
$$

if $n$ is odd, such that

$$
W_{\lambda}^{ \pm}(x)=\sum_{m=0}^{M-1} B_{m}^{ \pm}(\lambda) x^{-m}+O_{\Re(\lambda), M, n}\left(\mathfrak{C}(\lambda)^{2 M} x^{-M+\frac{n-1}{2}}\right),
$$

and

$$
E_{(\lambda, \delta)}^{ \pm}(x)=O_{\Re(\lambda), M, n}\left(\mathfrak{C}(\lambda)^{M} x^{-M}\right),
$$

for $x \geqslant \mathfrak{C}(\lambda)$. Furthermore, for $x \gg_{M, n} \mathfrak{C}(\lambda)^{2}$, we have

$$
W_{\lambda}^{ \pm}(x)=\sum_{m=0}^{M-1} B_{m}^{ \pm}(\lambda) x^{-m}+O_{M, n}\left(\mathfrak{C}(\lambda)^{2 M} x^{-M}\right),
$$

and

$$
E_{(\lambda, \delta)}^{ \pm}(x)=O_{n}\left(x^{-\frac{n-1}{2}} \exp \left(\pi \mathfrak{I}(\lambda)-2 \pi n \sin \left(\frac{1}{n} \pi\right) x\right)\right) .
$$

With the notations in Theorem 9.11 we have $W_{\lambda}^{ \pm}(x)=(2 \pi x)^{\frac{n-1}{2}} W^{ \pm}(2 \pi x ; \lambda)$ and $B_{m}^{ \pm}(\lambda)=$ $( \pm 2 \pi i)^{-m} B_{m}(\lambda)$. 


\section{Two Connection Formulae for $J_{(\mu, m)}(z)$}

In this section, we shall prove two formulae for $J_{(\mu, m)}(z)$ in connection with the two kinds of Bessel functions of the same rank and positive sign. These Bessel functions arise as solutions of Bessel differential equations in $\$ 11$ and their relations have been unraveled in $\$ 12.2$. Our motivation is based on the following self-evident identity for the rank-one example

$$
e(z+\bar{z})=e(z) e(\bar{z})
$$

15.1. The First Connection Formula. For $\varsigma \in\{+,-\}, \lambda \in \mathbb{C}^{n}$ and $l=1, \ldots, n$, we recollect the definition of the Bessel function of the first kind $J_{l}(z ; \varsigma, \lambda)$ by the following series of ascending powers of $z$ (see $\$ 11.1$ )

$$
J_{l}(z ; \varsigma, \lambda)=\sum_{m=0}^{\infty} \frac{\left(\varsigma i^{n}\right)^{m} z^{n\left(-\lambda_{l}+m\right)}}{\prod_{k=1}^{n} \Gamma\left(\lambda_{k}-\lambda_{l}+m+1\right)}, \quad z \in \mathbb{U} .
$$

Since the definition (15.1) is valid for any $\lambda \in \mathbb{C}^{n}$, the assumption $\lambda \in \mathbb{L}^{n-1}$ that we imposed in $\$ 11$ is rather superfluous. Also, we have the following formula in the same fashion as 3.7 in Lemma 3.5.

$$
J_{l}\left(z ; \varsigma, \lambda-\lambda \boldsymbol{e}^{n}\right)=z^{n \lambda} J_{l}(z ; \varsigma, \lambda)
$$

THeOREM 15.1. Let $(\boldsymbol{\mu}, \boldsymbol{m}) \in \mathbb{L}^{n-1} \times \mathbb{Z}^{n}$. We have

$$
J_{(\mu, \boldsymbol{m})}(z)=\left(2 \pi^{2}\right)^{n-1} \sum_{l=1}^{n} S_{l}(\boldsymbol{\mu}, \boldsymbol{m}) J_{l}\left(2 \pi z^{\frac{1}{n}} ;+, \boldsymbol{\mu}+\frac{1}{2} \boldsymbol{m}\right) J_{l}\left(2 \pi z^{\frac{1}{n}} ;+, \boldsymbol{\mu}-\frac{1}{2} \boldsymbol{m}\right),
$$

with $S_{l}(\boldsymbol{\mu}, \boldsymbol{m})=\prod_{k \neq l}( \pm i)^{m_{l}-m_{k}} / \sin \left(\pi\left(\mu_{l}-\mu_{k} \pm \frac{1}{2}\left(m_{l}-m_{k}\right)\right)\right)$. Here, $z^{\frac{1}{n}}$ is the principal $n$-th root of $z$, that is $\left(x e^{i \phi}\right)^{\frac{1}{n}}=x^{\frac{1}{n}} e^{\frac{1}{n} i \phi}$. The expression on the right hand side of 15.3 . is independent on the choice of the argument of $z$ modulo $2 \pi$. It is understood that the right hand side should be replaced by its limit if $(\boldsymbol{\mu}, \boldsymbol{m})$ is not generic with respect to the order $\leqslant$ on $\mathbb{C} \times \mathbb{Z}$ in the sense of Definition 3.1

Proof. Recall from 1.6, 1.7, 3.21, 3.43, that

$$
\begin{aligned}
J_{(\mu, \boldsymbol{m})}\left(x e^{i \phi}\right)= & (2 \pi)^{n-1} \sum_{m=-\infty}^{\infty} i^{\sum_{k=1}^{n}\left|m_{k}+m\right|} e^{i m \phi} \\
& \frac{1}{2 \pi i} \int_{\mathcal{C}_{\left(\mu, m+m e^{n}\right)}}\left(\prod_{l=1}^{n} \frac{\Gamma\left(s-\mu_{l}+\frac{1}{2}\left|m_{l}+m\right|\right)}{\Gamma\left(1-s+\mu_{l}+\frac{1}{2}\left|m_{l}+m\right|\right)}\right)\left((2 \pi)^{n} x\right)^{-2 s} d s .
\end{aligned}
$$

Assume first that $(\boldsymbol{\mu}, \boldsymbol{m})$ is generic with respect to the order $\leqslant$ on $\mathbb{C} \times \mathbb{Z}$. The sets of poles of the gamma factors in the above integral are $\left\{\mu_{l}-\frac{1}{2}\left|m_{l}+m\right|-\alpha\right\}_{\alpha \in \mathbb{N}}, l=1, \ldots, n$. With the generic assumption, the integrand has only simple poles. We left shift the integral contour of each integral in the series and collect the residues from these poles. The contribution from the residues at the poles of the $l$-th gamma factor is the following absolutely convergent double series,

$$
\begin{aligned}
(2 \pi)^{n-1} \sum_{m=-\infty}^{\infty} i^{\sum_{k=1}^{n}\left|m_{k}+m\right|} e^{i m \phi} \sum_{\alpha=0}^{\infty} \frac{(-)^{\alpha}\left((2 \pi)^{n} x\right)^{-2 \mu_{l}+\left|m_{l}+m\right|+2 \alpha}}{\alpha !\left(\alpha+\left|m_{l}+m\right|\right) !} \\
\prod_{k \neq l} \frac{\Gamma\left(\mu_{l}-\mu_{k}-\frac{1}{2}\left(\left|m_{l}+m\right|-\left|m_{k}+m\right|\right)-\alpha\right)}{\Gamma\left(1-\mu_{l}+\mu_{k}+\frac{1}{2}\left(\left|m_{l}+m\right|+\left|m_{k}+m\right|\right)+\alpha\right)} .
\end{aligned}
$$


Euler's reflection formula of the Gamma function converts this into

$$
\begin{aligned}
& \frac{\left(2 \pi^{2}\right)^{n-1}}{\prod_{k \neq l} i^{m_{l}-m_{k}} \sin \left(\pi\left(\mu_{l}-\mu_{k}-\frac{1}{2}\left(m_{l}-m_{k}\right)\right)\right)} \\
& \sum_{m=-\infty}^{\infty} i^{n\left|m_{l}+m\right|} e^{i m \phi} \sum_{\alpha=0}^{\infty} \frac{(-)^{n \alpha}\left((2 \pi)^{n} x\right)^{-2 \mu_{l}+\left|m_{l}+m\right|+2 \alpha}}{\prod_{k=1}^{n} \prod_{ \pm} \Gamma\left(1-\mu_{l}+\mu_{k}+\frac{1}{2}\left(\left|m_{l}+m\right| \pm\left|m_{k}+m\right|\right)+\alpha\right)}
\end{aligned}
$$

We now interchange the order of summations, truncate the sum over $m$ between $-m_{l}$ and $-m_{l}+1$ and make the change of indices $\beta=\alpha+\left|m_{l}+m\right|$. Observe that, regardless of the value of $m_{k}$, up to ordering, $\frac{1}{2}\left(\left|m_{l}+m\right|+\left|m_{k}+m\right|\right)$ and $\frac{1}{2}\left(\left|m_{l}+m\right|-\left|m_{k}+m\right|\right)$ equal to $\frac{1}{2}\left(m_{l}-m_{k}\right)$ and $\left|m_{l}+m\right|-\frac{1}{2}\left(m_{l}-m_{k}\right)$ when $m \geqslant-m_{l}+1$, or equal to $-\frac{1}{2}\left(m_{l}-m_{k}\right)$ and $\left|m_{l}+m\right|+\frac{1}{2}\left(m_{l}-m_{k}\right)$ when $m \leqslant-m_{l}$. Hence the double series in the expression above turns into

$$
\begin{aligned}
& \sum_{\alpha=0}^{\infty} \sum_{\beta=\alpha+1}^{\infty} \frac{i^{n(\alpha+\beta)} e^{i\left(\beta-\alpha-m_{l}\right) \phi}\left((2 \pi)^{n} x\right)^{-2 \mu_{l}+\alpha+\beta}}{\prod_{k=1}^{n} \Gamma\left(1-\mu_{l}+\mu_{k}+\frac{1}{2}\left(m_{l}-m_{k}\right)+\alpha\right) \Gamma\left(1-\mu_{l}+\mu_{k}-\frac{1}{2}\left(m_{l}-m_{k}\right)+\beta\right)} \\
& +\sum_{\alpha=0}^{\infty} \sum_{\beta=\alpha}^{\infty} \frac{i^{n(\alpha+\beta)} e^{i\left(\alpha-\beta-m_{l}\right) \phi}\left((2 \pi)^{n} x\right)^{-2 \mu_{l}+\alpha+\beta}}{\prod_{k=1}^{n} \Gamma\left(1-\mu_{l}+\mu_{k}-\frac{1}{2}\left(m_{l}-m_{k}\right)+\alpha\right) \Gamma\left(1-\mu_{l}+\mu_{k}+\frac{1}{2}\left(m_{l}-m_{k}\right)+\beta\right)},
\end{aligned}
$$

which is then equal to

$$
\sum_{\alpha=0}^{\infty} \sum_{\beta=0}^{\infty} \frac{i^{n(\alpha+\beta)} e^{i\left(\beta-\alpha-m_{l}\right) \phi}\left((2 \pi)^{n} x\right)^{-2 \mu_{l}+\alpha+\beta}}{\prod_{k=1}^{n} \Gamma\left(1-\mu_{l}+\mu_{k}+\frac{1}{2}\left(m_{l}-m_{k}\right)+\alpha\right) \Gamma\left(1-\mu_{l}+\mu_{k}-\frac{1}{2}\left(m_{l}-m_{k}\right)+\beta\right)} .
$$

This double series is clearly independent on the choice of $\phi$ modulo $2 \pi$, and splits exactly as the product

$$
J_{l}\left(2 \pi x^{\frac{1}{n}} e^{\frac{1}{n} i \phi} ;+, \boldsymbol{\mu}+\frac{1}{2} \boldsymbol{m}\right) J_{l}\left(2 \pi x^{\frac{1}{n}} e^{-\frac{1}{n} i \phi} ;+, \boldsymbol{\mu}-\frac{1}{2} \boldsymbol{m}\right) .
$$

This proves 15.3 in the case when $(\boldsymbol{\mu}, \boldsymbol{m})$ is generic. As for the nongeneric case, one just passes to the limit.

Q.E.D.

15.2. The Second Connection Formula. According to $\$ 11.3 .2$, Bessel functions of the second kind are solutions of Bessel equations defined according to their asymptotics at infinity. To remove the restriction $\lambda \in \mathbb{L}^{n-1}$ on the definition of $J(z ; \lambda ; \xi)$, with $\xi$ a $2 n$-th root of unity, we simply impose the additional condition

$$
J\left(z ; \lambda-\lambda \boldsymbol{e}^{n} ; \xi\right)=z^{n \lambda} J(z ; \lambda ; \xi) .
$$

Remark 15.2. Let $\xi$ be an $n$-th root of $\varsigma 1$. We may also use the following formula as an alternative definition of $J(z ; \lambda ; \xi)$ (compare Corollary 12.5.

$$
J(z ; \lambda ; \xi)=\sqrt{n}\left(\frac{\pi}{2}\right)^{\frac{n-1}{2}}(-i \xi)^{\frac{n-1}{2}+|\lambda|} \sum_{l=1}^{n}(i \bar{\xi})^{n \lambda_{l}} S_{l}(\lambda) J_{l}(z ; \varsigma, \lambda) .
$$

where $(-i \xi)^{\frac{n-1}{2}+|\lambda|}=e^{\left(\frac{n-1}{2}+|\lambda|\right)\left(-\frac{1}{2} \pi i+i \arg \xi\right)}$ and $(i \bar{\xi})^{n \lambda_{l}}=e^{\frac{1}{2} \pi i n \lambda_{l}-i n \lambda_{l} \arg \xi}$ by convention, and $S_{l}(\lambda)=1 / \prod_{k \neq l} \sin \left(\pi\left(\lambda_{l}-\lambda_{k}\right)\right)$.

Given an integer $a$, define $\xi_{a, j}=e^{2 \pi i \frac{j+a-1}{n}}, j=1, \ldots, n$. For $d=0,1, \ldots, n-1$ and $l=$ $1, \ldots, n$, let $\sigma_{l, d}(\lambda)$ denote the elementary symmetric polynomial in $e^{-2 \pi i \lambda_{1}}, \ldots, \widehat{e^{-2 \pi i \lambda_{l}}}, \ldots$ 
$e^{-2 \pi i \lambda_{n}}$ of degree $d$. It follows from Corollary 12.7 that

$$
\begin{aligned}
J_{l}(z ;+, \lambda)=\frac{e^{\frac{3}{4} \pi i((n-1)+2|\lambda|)}}{\sqrt{n}(2 \pi)^{\frac{n-1}{2}}} e^{\pi i\left(\frac{1}{2} n+2 a-2\right) \lambda_{l}} \\
\sum_{j=1}^{n}(-)^{n-j} \xi_{a, j}^{-\frac{n-1}{2}-|\lambda|} \sigma_{l, n-j}(\lambda) J\left(z ; \lambda ; \xi_{a, j}\right) .
\end{aligned}
$$

In addition, we shall require the definition

$$
\tau_{l}(\lambda)=\prod_{k \neq l}\left(e^{-2 \pi i \lambda_{m}}-e^{-2 \pi i \lambda_{k}}\right)=(-2 i)^{n-1} e^{-\pi i|\lambda|} e^{-\pi i(n-2) \lambda_{l}} \prod_{k \neq l} \sin \left(\pi\left(\lambda_{l}-\lambda_{k}\right)\right) .
$$

Note that the product of sines is equal to $1 / S_{l}(\lambda)$.

We introduce the column vectors of the two kinds of Bessel functions

$$
X(z ; \lambda)=\left(J_{l}(z ;+, \lambda)\right)_{l=1}^{n}, \quad Y_{a}(z ; \lambda)=\left(J\left(z ; \lambda ; \xi_{a, j}\right)\right)_{j=1}^{n},
$$

and the matrices

$$
\begin{aligned}
\Sigma(\lambda) & =\left(\sigma_{l, n-j}(\lambda)\right)_{l, j=1}^{n}, \\
E_{a}(\lambda) & =\operatorname{diag}\left(e^{\pi i\left(\frac{1}{2} n+2 a-2\right) \lambda_{l}}\right)_{l=1}^{n}, \quad D_{a}(\lambda)=\operatorname{diag}\left((-)^{n-j} \xi_{a, j}^{-\frac{n-1}{2}-|\lambda|}\right)_{j=1}^{n} .
\end{aligned}
$$

Then the formula 15.6 may be written as

$$
X(z ; \lambda)=\frac{e^{\frac{3}{4} \pi i((n-1)+2|\lambda|)}}{\sqrt{n}(2 \pi)^{\frac{n-1}{2}}} \cdot E_{a}(\lambda) \Sigma(\lambda) D_{a}(\lambda) Y_{a}(z ; \lambda) .
$$

We now formulate 15.3 as

$$
J_{(\mu, \boldsymbol{m})}(z)=(-)^{|\boldsymbol{m}|} e^{-\frac{1}{2} \pi i(n-1)}\left(4 \pi^{2}\right)^{n-1} \cdot{ }^{t} X\left(2 \pi z^{\frac{1}{n}} ; \lambda_{(\mu, \boldsymbol{m})}^{+}\right) S_{(\mu, \boldsymbol{m})} X\left(2 \pi \bar{z}^{\frac{1}{n}} ; \lambda_{(\mu, \boldsymbol{m})}^{-}\right) \text {, }
$$

with $\boldsymbol{\lambda}_{(\mu, \boldsymbol{m})}^{ \pm}=\boldsymbol{\mu} \pm \frac{1}{2} \boldsymbol{m}$ and

$$
S_{(\mu, \boldsymbol{m})}=\operatorname{diag}\left(\tau_{l}\left(\lambda_{(\boldsymbol{\mu}, \boldsymbol{m})}^{ \pm}\right)^{-1} e^{-\pi i\left((n-2) \mu_{l} \mp m_{l}\right)}\right)_{l=1}^{n} .
$$

We insert into 15.8 the formulae of $X\left(2 \pi z^{\frac{1}{n}} ; \lambda_{(\mu, \boldsymbol{m})}^{+}\right)$and $X\left(2 \pi z^{\frac{1}{n}} ; \lambda_{(\mu, \boldsymbol{m})}^{-}\right)$given by 15.7), with $\lambda=\lambda_{(\mu, m)}^{+}, a=0$ in the former and $\lambda=\lambda_{(\mu, m)}^{-}, a=1-r$, for $r=0,1, \ldots, n$, in the latter. Then follows the formula

$$
\begin{aligned}
& J_{(\mu, \boldsymbol{m})}(z)=(-)^{(n-1)+|\boldsymbol{m}|} \frac{(2 \pi)^{n-1}}{n} \\
& { }^{t} Y_{0}\left(2 \pi z^{\frac{1}{n}} ; \lambda_{(\mu, \boldsymbol{m})}^{+}\right) D_{0}\left(\lambda_{(\mu, \boldsymbol{m})}^{+}\right){ }^{t} \Sigma_{(\mu, \boldsymbol{m})} R_{(\mu, \boldsymbol{m})} \Sigma_{(\mu, \boldsymbol{m})} D_{1-r}\left(\lambda_{(\mu, \boldsymbol{m})}^{-}\right) Y_{1-r}\left(2 \pi z^{\frac{1}{n}} ; \lambda_{(\mu, \boldsymbol{m})}^{-}\right),
\end{aligned}
$$

where

$$
\begin{aligned}
& \Sigma_{(\mu, \boldsymbol{m})}=\Sigma\left(\lambda_{(\mu, \boldsymbol{m})}^{+}\right)=\Sigma\left(\lambda_{(\mu, \boldsymbol{m})}^{-}\right) \\
& R_{(\mu, \boldsymbol{m})}=E_{0}\left(\lambda_{(\mu, \boldsymbol{m})}^{+}\right) S_{(\mu, \boldsymbol{m})} E_{1-r}\left(\lambda_{(\mu, \boldsymbol{m})}^{-}\right)=\operatorname{diag}\left(\tau_{l}\left(\lambda_{(\mu, \boldsymbol{m})}^{ \pm}\right)^{-1} e^{-2 \pi i r \lambda_{(\mu, m), l}^{ \pm}}\right)_{l=1}^{n}
\end{aligned}
$$

We are therefore reduced to computing the matrix ${ }^{t} \Sigma_{(\mu, m)} R_{(\mu, m)} \Sigma_{(\mu, m)}$. For this, we have the following lemma. 
Lemma 15.3. Let $\boldsymbol{x}=\left(x_{1}, \ldots, x_{n}\right) \in \mathbb{C}^{n}$ be a generic $n$-tuple in the sense that all its components are distinct. Let $\sigma_{l, d}$, respectively $\sigma_{d}$, denote the elementary symmetric polynomial in $x_{1}, \ldots, \hat{x}_{l}, \ldots, x_{n}$, respectively $x_{1}, \ldots, x_{n}$, of degree $d$, and let $\tau_{l}=\prod_{h \neq l}\left(x_{l}-x_{h}\right)$. Define the matrices $\Sigma=\left(\sigma_{l, n-j}\right)_{l, j=1}^{n}, X=\operatorname{diag}\left(x_{l}\right)_{l=1}^{n}$ and $T=\operatorname{diag}\left(\tau_{l}^{-1}\right)_{l=1}^{n}$. Then, for any $r=0,1, \ldots, n$, the matrix ${ }^{t} \Sigma X^{r} T \Sigma$ can be written as

$$
\left(\begin{array}{cc}
(-)^{n-r} A & 0 \\
0 & (-)^{n-r+1} B
\end{array}\right)
$$

where

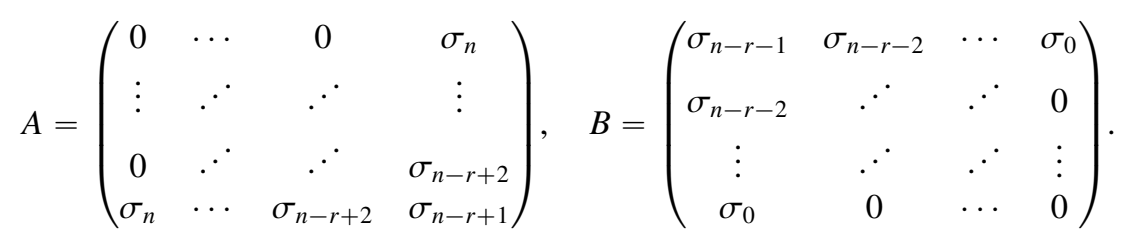

More precisely, the $(k, j)$-th entry $a_{k, j}, k, j=1, \ldots, r$, of $A$ is given by

$$
a_{k, j}= \begin{cases}\sigma_{n+r-k-j+1} & \text { if } k+j \geqslant r+1, \\ 0 & \text { if otherwise }\end{cases}
$$

whereas the $(k, j)$-th entry $b_{k, j}, k, j=1, \ldots, n-r$, of $B$ is given by

$$
b_{k, j}= \begin{cases}\sigma_{n-r-k-j+1} & \text { if } k+j \leqslant n-r+1, \\ 0 & \text { if otherwise. }\end{cases}
$$

Proof. Appealing to the Lagrange interpolation formula, we find in Lemma 12.6 that the inverse of $T \Sigma$ is equal to the matrix $U=\left((-)^{n-j} x_{l}^{j-1}\right)_{j, l=1}^{n}$. Therefore, it suffices to show that

$$
{ }^{t} \Sigma X^{r}=\left(\begin{array}{cc}
(-)^{n-r} A & 0 \\
0 & (-)^{n-r+1} B
\end{array}\right) U .
$$

This is equivalent to the following two collections of identities,

$$
\begin{gathered}
\sum_{j=r-k+1}^{r}(-)^{r+j} \sigma_{n+r-k-j+1} x_{l}^{j-1}=\sigma_{l, n-k} x_{l}^{r}, \quad k=1, \ldots, r, \\
\sum_{j=1}^{n-r-k+1}(-)^{j-1} \sigma_{n-r-k-j+1} x_{l}^{r+j-1}=\sigma_{l, n-r-k} x_{l}^{r}, \quad k=1, \ldots, n-r,
\end{gathered}
$$

which are further equivalent to

$$
\begin{aligned}
\sum_{j=1}^{k}(-)^{k+j} \sigma_{n-j+1} x_{l}^{j-k-1} & =\sigma_{l, n-k}, \quad k=1, \ldots, r, \\
\sum_{j=1}^{k}(-)^{j-1} \sigma_{k-j} x_{l}^{j-1} & =\sigma_{l, k-1}, \quad k=1, \ldots, n-r .
\end{aligned}
$$

The last two identities can be easily seen, actually for all $k=1, \ldots, n$, from computing the coefficients of $x^{k-1}$ and $x^{2 n-k}$ on the two sides of

$$
\prod_{h \neq l}\left(x-x_{h}\right)=\left(\sum_{p=0}^{\infty} x_{l}^{p} x^{-p-1}\right) \prod_{h=1}^{n}\left(x-x_{h}\right),
$$




$$
\left(x^{n}-x_{l}^{n}\right) \prod_{h \neq l}\left(x-x_{h}\right)=\left(\sum_{p=1}^{n} x_{l}^{p-1} x^{n-p}\right) \prod_{h=1}^{n}\left(x-x_{h}\right),
$$

respectively.

Q.E.D.

Applying Lemma 15.3 with $x_{l}=e^{-2 \pi i \lambda_{(\mu, m), l}^{ \pm}}=(-)^{m_{l}} e^{-2 \pi i \mu_{l}}$ to the formula 15.9], we arrive at the following theorem.

Theorem 15.4. Let $(\boldsymbol{\mu}, \boldsymbol{m}) \in \mathbb{L}^{n-1} \times \mathbb{Z}^{n}$ and $r \in\{0,1, . ., n\}$. Define $\xi_{j}=e^{2 \pi i \frac{j-1}{n}}$, $\zeta_{j}=e^{2 \pi i \frac{j-r}{n}}$, and denote by $\sigma_{(\mu, \boldsymbol{m})}^{d}$ the elementary symmetric polynomial in $(-)^{m_{1}} e^{-2 \pi i \mu_{1}}$, $\ldots,(-)^{m_{n}} e^{-2 \pi i \mu_{n}}$ of degree $d$, with $j=1, \ldots, n$ and $d=0,1, \ldots, n$. Then we have

$$
\begin{aligned}
J_{(\mu, \boldsymbol{m})}(z)=(-)^{|\boldsymbol{m}|} \frac{(2 \pi)^{n-1}}{n} \sum_{\substack{k, j=1, \ldots, r \\
k+j \geqslant r+1}} C_{k, j}(\boldsymbol{\mu}, \boldsymbol{m}) \\
\quad J\left(2 \pi z^{\frac{1}{n}} ; \boldsymbol{\mu}+\frac{1}{2} \boldsymbol{m} ; \xi_{k}\right) J\left(2 \pi \bar{z}^{\frac{1}{n}} ; \boldsymbol{\mu}-\frac{1}{2} \boldsymbol{m} ; \zeta_{j}\right) \\
+(-)^{|\boldsymbol{m}|} \frac{(2 \pi)^{n-1}}{n} \sum_{\substack{k, j=1, \ldots, n-r \\
k+j \leqslant n-r+1}} D_{k, j}(\boldsymbol{\mu}, \boldsymbol{m}) \\
\quad J\left(2 \pi z^{\frac{1}{n}} ; \boldsymbol{\mu}+\frac{1}{2} \boldsymbol{m} ; \xi_{r+k}\right) J\left(2 \pi \bar{z}^{\frac{1}{n}} ; \boldsymbol{\mu}-\frac{1}{2} \boldsymbol{m} ; \zeta_{r+j}\right) .
\end{aligned}
$$

with

$$
\begin{aligned}
& C_{k, j}(\boldsymbol{\mu}, \boldsymbol{m})=(-)^{r+k+j+1} \xi_{k}^{-\frac{n-1}{2}-\frac{1}{2}|\boldsymbol{m}|} \zeta_{j}^{-\frac{n-1}{2}+\frac{1}{2}|\boldsymbol{m}|} \sigma_{(\boldsymbol{\mu}, \boldsymbol{m})}^{n+-k-j+1}, \\
& D_{k, j}(\boldsymbol{\mu}, \boldsymbol{m})=(-)^{r+k+j} \xi_{r+k}^{-\frac{n-1}{2}-\frac{1}{2}|\boldsymbol{m}|} \zeta_{r+j}^{-\frac{n-1}{2}+\frac{1}{2}|\boldsymbol{m}|} \sigma_{(\boldsymbol{\mu}, \boldsymbol{m})}^{n-r-k-j+1} .
\end{aligned}
$$

Lemma 15.5. We retain the notations in Theorem 15.4 Moreover, we define $\mathfrak{I}(\boldsymbol{\mu})=$ $\max \left\{\left|\mathfrak{I} \mathfrak{m} \mu_{l}\right|\right\}$.

(1.1). For $k=1, \ldots, r$, we have $C_{k, r-k+1}(\boldsymbol{\mu}, \boldsymbol{m})=\left(-\bar{\xi}_{k}\right)^{|\boldsymbol{m}|}$.

(1.2). Let $k, j=1, \ldots, r$ be such that $k+j \geqslant r+2$. Denote $p=k+j-r-1$. We have the estimate

$$
\left|C_{k, j}(\boldsymbol{\mu}, \boldsymbol{m})\right| \leqslant\left(\begin{array}{c}
n \\
p
\end{array}\right) \exp (2 \pi \min \{n-p, p\} \Im(\boldsymbol{\mu})) .
$$

(2.1). For $k=1, \ldots, n-r$, we have $D_{k, n-r-k+1}(\boldsymbol{\mu}, \boldsymbol{m})=\left(-\bar{\xi}_{k+r}\right)^{|\boldsymbol{m}|}$.

(2.2). Let $k, j=1, \ldots, n-r$ be such that $k+j \leqslant n-r$. Denote $p=n-r-k-j+1$. We have the estimate

$$
\left|D_{k, j}(\boldsymbol{\mu}, \boldsymbol{m})\right| \leqslant\left(\begin{array}{l}
n \\
p
\end{array}\right) \exp (2 \pi \min \{n-p, p\} \Im(\boldsymbol{\mu})) .
$$

\subsection{The Rank-Two Case.}

Example 15.6. Let $\mu \in \mathbb{C}$ and $m \in \mathbb{Z}$.

If we define

$$
J_{\mu, m}(z)=J_{-2 \mu-\frac{1}{2} m}(z) J_{-2 \mu+\frac{1}{2} m}(\bar{z}),
$$

then

$$
J_{(\mu,-\mu, m, 0)}(z)=\left\{\begin{array}{l}
\frac{2 \pi^{2}}{\sin (2 \pi \mu)}[\sqrt{z}]^{-m}\left(J_{\mu, m}(4 \pi \sqrt{z})-J_{-\mu,-m}(4 \pi \sqrt{z})\right) \text { if } m \text { is even } \\
\frac{2 \pi^{2} i}{\cos (2 \pi \mu)}[\sqrt{z}]^{-m}\left(J_{\mu, m}(4 \pi \sqrt{z})+J_{-\mu,-m}(4 \pi \sqrt{z})\right) \text { if } m \text { is odd }
\end{array}\right.
$$


which should be interpreted in the way as in Theorem 15.1. We remark that the generic case is when $4 \mu \notin 2 \mathbb{Z}+m$.

On the other hand, using the connection formulae ([Wat $3.61(1,2)])$

$$
J_{v}(z)=\frac{H_{v}^{(1)}(z)+H_{v}^{(2)}(z)}{2}, \quad J_{-v}(z)=\frac{e^{\pi i v} H_{v}^{(1)}(z)+e^{-\pi i v} H_{v}^{(2)}(z)}{2},
$$

one obtains

$$
J_{(\mu,-\mu, m, 0)}(z)=\pi^{2} i[\sqrt{z}]^{-m}\left(e^{2 \pi i \mu} H_{\mu, m}^{(1)}(4 \pi \sqrt{z})+(-)^{m+1} e^{-2 \pi i \mu} H_{\mu, m}^{(2)}(4 \pi \sqrt{z})\right),
$$

with the definition

$$
H_{\mu, m}^{(1,2)}(z)=H_{2 \mu+\frac{1}{2} m}^{(1,2)}(z) H_{2 \mu-\frac{1}{2} m}^{(1,2)}(\bar{z}) .
$$

\section{The Asymptotic Expansion of $J_{(\mu, m)}(z)$}

In the following, we shall present the asymptotic expansion of $J_{(\mu, m)}(z)$.

First of all, we have the following proposition on the asymptotic expansion of $J(z ; \lambda ; \xi)$, which is in substance Theorem 11.24 .

Proposition 16.1. Let $\lambda \in \mathbb{C}^{n}$ and define $\mathfrak{C}(\lambda)=\max \left\{\left|\lambda_{l}-\frac{1}{n}\right| \lambda||+1\right\}$. Let $\xi$ be a $2 n$-th root of unity. For a small positive constant $\vartheta$, say $0<\vartheta<\frac{1}{2} \pi$, we define the sector

$$
\mathbb{S}_{\xi}^{\prime}(\vartheta)=\left\{z:|\arg z-\arg (i \bar{\xi})|<\pi+\frac{\pi}{n}-\vartheta\right\} .
$$

For a positive integer $A$, we have the asymptotic expansion

$$
J(z ; \lambda ; \xi)=e^{i n \xi z} z^{-\frac{n-1}{2}-|\lambda|}\left(\sum_{\alpha=0}^{A-1}(i \xi)^{-\alpha} B_{\alpha}\left(\lambda-\frac{1}{n}|\lambda| \boldsymbol{e}^{n}\right) z^{-\alpha}+O_{A, \vartheta, n}\left(\mathfrak{C}(\lambda)^{2 A}|z|^{-A}\right)\right)
$$

for all $z \in \mathbb{S}_{\xi}^{\prime}(\vartheta)$ such that $|z| \gg_{A, \vartheta, n} \mathfrak{C}(\lambda)^{2}$. Here $B_{\alpha}(\lambda)$ is a certain symmetric polynomial function in $\lambda \in \mathbb{L}^{n-1}$ of degree $2 \alpha$, with $B_{0}(\lambda)=1$.

Lemma 16.2. Let $r$ be a positive integer. Suppose that either $n=2 r$ or $n=2 r-1$. Put $\vartheta_{n}=\frac{1}{n} \pi$ if $n=2 r$ and $\vartheta_{n}=\frac{1}{2 n} \pi$ if $n=2 r-1$. For a given constant $0<\vartheta<\vartheta_{n}$ define the sector

$$
\mathbb{S}_{n}(\vartheta)= \begin{cases}\left\{z:-\frac{\pi}{2}-\frac{\pi}{n}+\vartheta<\arg z<-\frac{\pi}{2}+\frac{3 \pi}{n}-\vartheta\right\} & \text { if } n=2 r, \\ \left\{z:-\frac{\pi}{2}-\frac{\pi}{n}+\vartheta<\arg z<-\frac{\pi}{2}+\frac{2 \pi}{n}-\vartheta\right\} & \text { if } n=2 r-1,\end{cases}
$$

Let $(\boldsymbol{\mu}, \boldsymbol{m}) \in \mathbb{L}^{n-1} \times \mathbb{Z}^{n}$ and define $\mathfrak{C}(\boldsymbol{\mu}, \boldsymbol{m})=\max \left\{\left|\mu_{l}\right|+1,\left|m_{l}-\frac{1}{n}\right| \boldsymbol{m}||+1\right\}$. Define $\xi_{j}=e^{2 \pi i \frac{j-1}{n}}$ and $\zeta_{j}=e^{2 \pi i \frac{j-r}{n}}$ for $j=1, \ldots, n$. Then, for any $z \in \mathbb{S}_{n}(\vartheta)$ such that $|z| \gg_{A, \vartheta, n}$ $\mathfrak{C}(\boldsymbol{\mu}, \boldsymbol{m})^{2}$, we have

$$
\begin{aligned}
J(2 \pi z ; \boldsymbol{\mu}+ & \left.\frac{1}{2} \boldsymbol{m} ; \xi_{k}\right) J\left(2 \pi \bar{z} ; \boldsymbol{\mu}-\frac{1}{2} \boldsymbol{m} ; \zeta_{j}\right)=\frac{e\left(n\left(\xi_{k} z+\zeta_{j} \bar{z}\right)\right)}{(2 \pi)^{n-1}|z|^{n-1}[z]^{|\boldsymbol{m}|}} \\
& \left(\sum_{\substack{\alpha, \beta=0, \ldots, A-1 \\
\alpha+\beta \leqslant A-1}}\left(i \xi_{k}\right)^{-\alpha}\left(i \zeta_{j}\right)^{-\beta} B_{\alpha, \beta}(\boldsymbol{\mu}, \boldsymbol{m}) z^{-\alpha} \bar{z}^{-\beta}+O_{A, \vartheta, n}\left(\mathfrak{C}(\boldsymbol{\mu}, \boldsymbol{m})^{2 A}|z|^{-A}\right)\right),
\end{aligned}
$$


with

$$
B_{\alpha, \beta}(\boldsymbol{\mu}, \boldsymbol{m})=(2 \pi)^{-\alpha-\beta} B_{\alpha}\left(\boldsymbol{\mu}+\frac{1}{2} \boldsymbol{m}-\frac{1}{2 n}|\boldsymbol{m}| \boldsymbol{e}^{n}\right) B_{\beta}\left(\boldsymbol{\mu}-\frac{1}{2} \boldsymbol{m}+\frac{1}{2 n}|\boldsymbol{m}| \boldsymbol{e}^{n}\right), \quad \alpha, \beta \in \mathbb{N},
$$

where $B_{\alpha}(\lambda)$ is the polynomial function in $\lambda$ of degree $2 \alpha$ given in Proposition 16.1 .

Proof. Recall that, for an integer $a$, we defined $\xi_{a, j}=e^{2 \pi i \frac{j+a-1}{n}}$. Note that $\xi_{j}=\xi_{0, j}$ and $\zeta_{j}=\xi_{1-r, j}$. It is clear that

$$
\bigcap_{j=1}^{n} \mathbb{S}_{\xi_{a, j}}^{\prime}(\vartheta)=\left\{z:-\frac{\pi}{2}-\frac{2 a+1}{n} \pi+\vartheta<\arg z<-\frac{\pi}{2}-\frac{2 a-3}{n} \pi-\vartheta\right\} .
$$

We denote this sector by $\mathbb{S}_{a}^{\prime}(\vartheta)$. Observe that, when $n=2 r$ or $2 r-1$, the intersection $\mathbb{S}_{0}^{\prime}(\vartheta) \cap \overline{\mathbb{S}_{1-r}^{\prime}(\vartheta)}$ is exactly the sector $\mathbb{S}_{n}(\vartheta)$. In other words, for all $j=1, \ldots, n, z \in \mathbb{S}_{\xi_{j}}^{\prime}(\vartheta)$ and $\bar{z} \in \mathbb{S}_{\zeta_{j}}^{\prime}(\vartheta)$ both hold if $z \in \mathbb{S}_{n}(\vartheta)$. Therefore, Proposition 16.1 can be applied to yield the asymptotic expansion of $J\left(2 \pi z ; \boldsymbol{\mu}+\frac{1}{2} \boldsymbol{m} ; \xi_{k}\right) J\left(2 \pi \bar{z} ; \boldsymbol{\mu}-\frac{1}{2} \boldsymbol{m} ; \zeta_{j}\right)$ as above. Q.E.D.

REMARK 16.3. In view of our choice of $\vartheta$, the sector $\mathbb{S}_{n}(\vartheta)$ is of angle at least $\frac{2}{n} \pi$, and therefore the sector $\mathbb{S}_{n}(\vartheta)^{n}=\left\{z^{n}: z \in \mathbb{S}_{n}(\vartheta)\right\}$ covers the whole $\mathbb{C} \backslash\{0\}$.

LeMma 16.4. Let notations be as in Lemma 16.2

(1.1). For $k=1, \ldots, r$, we have

$$
\mathfrak{I m}\left(\xi_{k} z+\zeta_{r-k+1} \bar{z}\right)=0 .
$$

(1.2). Let $k, j=1, \ldots, r$ be such that $k+j \geqslant r+2$. For any $z \in \mathbb{S}_{n}(\vartheta)$, we have

$$
\mathfrak{I m}\left(\xi_{k} z+\zeta_{j} \bar{z}\right) \geqslant 2 \sin \left(\frac{k+j-r-1}{n} \pi\right) \sin \vartheta \cdot|z| .
$$

(2.1). For $k=1, \ldots, n-r$, we have

$$
\mathfrak{I m}\left(\xi_{k+r} z+\zeta_{n-k+1} \bar{z}\right)=0 .
$$

(2.2). Let $k, j=1, \ldots, n-r$ be such that $k+j \leqslant n-r$. For any $z \in \mathbb{S}_{n}(\vartheta)$, we have

$\mathfrak{I m}\left(\xi_{k+r} z+\zeta_{j+r} \bar{z}\right) \geqslant \begin{cases}2 \sin \left(\frac{n-r-k-j+1}{n} \pi\right) \sin \vartheta \cdot|z|, & \text { if } n=2 r, \\ 2 \sin \left(\frac{n-r-k-j+1}{n} \pi\right) \sin \left(\frac{\pi}{n}+\vartheta\right) \cdot|z|, & \text { if } n=2 r-1 .\end{cases}$

Proof. We shall only prove (1.1) and (1.2) in the case $n=2 r$. The other cases follow in exactly the same way.

Write $z=x e^{i \phi}$. Since

$$
\begin{aligned}
\xi_{k} z+\zeta_{j} \bar{z} & =x e^{2 \pi i \frac{k-1}{2 r}+i \phi}+x e^{2 \pi i \frac{j-r}{2 r}-i \phi} \\
& =x e^{\pi i \frac{k+j-r-1}{2 r}}\left(e^{\pi i \frac{k-j+r-1}{2 r}+i \phi}+e^{-\pi i \frac{k-j+r-1}{2 r}-i \phi}\right),
\end{aligned}
$$

(1.1) is then obvious (we also note that $\zeta_{r-k+1}=\bar{\xi}_{k}$ ), whereas (1.2) is equivalent to

$$
\cos \left(\frac{k-j+r-1}{2 r} \pi+\phi\right) \geqslant \sin \vartheta
$$

Observe that the condition $z \in \mathbb{S}_{2 r}(\vartheta)$ amounts to

$$
\left|\phi+\frac{\pi}{2}-\frac{\pi}{2 r}\right|<\frac{\pi}{r}-\vartheta
$$


Moreover, under our assumptions on $k$ and $j$ in (1.2), one has $|k-j| \leqslant r-2$. Consequently, these yield the following estimate

$$
\left|\frac{k-j+r-1}{2 r} \pi+\phi\right| \leqslant \frac{r-2}{2 r} \pi+\frac{\pi}{r}-\vartheta=\frac{\pi}{2}-\vartheta .
$$

Thus 16.1 is proven.

Q.E.D.

REMARK 16.5. In the cases other than those listed in Lemma 16.4 $\mathfrak{I m}\left(\xi_{k} z+\zeta_{j} \bar{z}\right)$ can not always be nonnegative for all $z \in \mathbb{S}_{n}(\vartheta)$. Fortunately, these cases are excluded from the second connection formula for $J_{(\mu, m)}(z)$ in Theorem 15.4 .

Now the asymptotic expansion of $J_{(\mu, m)}(z)$ can be readily established using Theorem 15.4 along with Lemma $15.5,16.2$ and 16.4 .

THeOREM 16.6. Denote by $\mathbb{X}_{n}$ the set of $n$-th roots of unity. Let $(\boldsymbol{\mu}, \boldsymbol{m}) \in \mathbb{L}^{n-1} \times \mathbb{Z}^{n}$ and define $\mathfrak{C}(\boldsymbol{\mu}, \boldsymbol{m})=\max \left\{\left|\mu_{l}\right|+1,\left|m_{l}-\frac{1}{n}\right| \boldsymbol{m}||+1\right\}$. Let $A$ be a positive integer. Then

$$
\begin{array}{r}
J_{(\mu, \boldsymbol{m})}\left(z^{n}\right)=\sum_{\xi \in \mathbb{X}_{n}} \frac{e(n(\xi z+\overline{\xi z}))}{n|z|^{n-1}[\xi z]^{|\boldsymbol{m}|}}\left(\sum_{\begin{array}{c}
\alpha, \beta=0, \ldots, A-1 \\
\alpha+\beta \leqslant A-1
\end{array}} i^{-\alpha-\beta} \xi^{-\alpha+\beta} B_{\alpha, \beta}(\boldsymbol{\mu}, \boldsymbol{m}) z^{-\alpha} \bar{z}^{-\beta}\right) \\
+O_{A, n}\left(\mathfrak{C}(\boldsymbol{\mu}, \boldsymbol{m})^{2 A}|z|^{-A-n+1}\right),
\end{array}
$$

if $|z| \gg_{A, n} \mathfrak{C}(\boldsymbol{\mu}, \boldsymbol{m})^{2}$, with the coefficient $B_{\alpha, \beta}(\boldsymbol{\mu}, \boldsymbol{m})$ given in Lemma 16.2

We may also prove the following elaborate version of Theorem 16.6

Theorem 16.7. Let notations be as in Lemma 16.2 and Theorem 16.6 Fix the angle $\vartheta$, say $\vartheta=\frac{1}{2} \vartheta_{n}$. Let $\mathfrak{I}(\boldsymbol{\mu})=\max \left\{\left|\mathfrak{I m} \mu_{l}\right|\right\}$. Then we may write

$$
J_{(\mu, \boldsymbol{m})}\left(z^{n}\right)=\sum_{\xi \in \mathbb{X}_{n}} \frac{e(n(\xi z+\overline{\xi z}))}{n|z|^{n-1}[\xi z]^{|\boldsymbol{m}|}} W_{(\mu, \boldsymbol{m})}(z, \xi)+E_{(\mu, \boldsymbol{m})}(z),
$$

such that

$$
W_{(\boldsymbol{\mu}, \boldsymbol{m})}(z, \xi)=\sum_{\substack{\alpha, \beta=0, \ldots, A-1 \\ \alpha+\beta \leqslant A-1}} i^{-\alpha-\beta} \xi^{-\alpha+\beta} B_{\alpha, \beta}(\boldsymbol{\mu}, \boldsymbol{m}) z^{-\alpha} \bar{z}^{-\beta}+O_{A, n}\left(\mathfrak{C}(\boldsymbol{\mu}, \boldsymbol{m})^{2 A}|z|^{-A}\right),
$$

and

$$
E_{(\mu, \boldsymbol{m})}(z)=O_{n}\left(|z|^{-n+1} \exp \left(2 \pi \mathfrak{I}(\boldsymbol{\mu})-4 \pi n \sin \left(\frac{1}{n} \pi\right) \sin \vartheta \cdot|z|\right)\right),
$$

for $z \in \mathbb{S}_{n}(\vartheta)$ with $|z| \gg_{A, n} \mathfrak{C}(\boldsymbol{\mu}, \boldsymbol{m})^{2}$. Moreover, $E_{(\mu, \boldsymbol{m})}(z) \equiv 0$ when $n=1,2$. 



\section{CHAPTER 4}

\section{Hankel Transforms and Bessel Kernels in Representation Theory}

This last chapter is devoted to the representation theoretic investigations of Hankel transforms for $\mathrm{GL}_{n}(\mathbb{F})$ and Bessel kernels for $\mathrm{GL}_{2}(\mathbb{F})$, with $\mathbb{F}=\mathbb{R}$ or $\mathbb{C}$.

\section{Hankel Transforms from the Representation Theoretic Viewpoint}

We shall start with a brief review of Hankel transforms over an Archimedean local field] in the work of Ichino and Templier [IT] on the Voronoï summation formula. For the theory of $L$-functions and local functional equations over a local field the reader is referred to Cogdell's survey $[\mathbf{C o g}]$. We shall then study Hankel transforms using the Langlands classification. For this, Knapp's article [Kna] is used as our reference, with some changes of notations for our convenience.

Let $\mathbb{F}$ be an Archimedean local field with normalized absolute value \|\|$=\|\|_{\mathbb{F}}$ defined as in $\$ 1.2$, and let $\psi$ be a given additive character on $\mathbb{F}$. For $s \in \mathbb{C}$ let $\omega_{s}$ denote the character $\omega_{s}(x)=\|x\|^{s}$. Let $\eta(x)=\operatorname{sgn}(x)$ for $x \in \mathbb{R}^{\times}$and $\eta(z)=[z]$ for $z \in \mathbb{C}^{\times}$.

Suppose for the moment $n \geqslant 2$. Let $\pi$ be an infinite dimensional irreducible admissible generic representation of $\mathrm{GL}_{n}(\mathbb{F})^{\mathbb{1}}$, and $\mathscr{W}(\pi, \psi)$ be the $\psi$-Whittaker model of $\pi$. Denote by $\omega_{\pi}$ the central character of $\pi$. Recall that the $\gamma$-factor $\gamma(s, \pi, \psi)$ of $\pi$ is given by

$$
\gamma(s, \pi, \psi)=\epsilon(s, \pi, \psi) \frac{L(1-s, \tilde{\pi})}{L(s, \pi)}
$$

where $\tilde{\pi}$ is the contragradient representation of $\pi, \epsilon(s, \pi, \psi)$ and $L(s, \pi)$ are the $\epsilon$-factor and the $L$-function of $\pi$ respectively.

To a smooth compactly supported function $w$ on $\mathbb{F}^{\times}$we associate a dual function $\widetilde{w}$ on $\mathbb{F}^{\times}$defined by [IT $\left.(1.1)\right]$,

$$
\begin{aligned}
& \int_{\mathbb{F} \times} \tilde{w}(x) \chi(x)^{-1}\|x\|^{s-\frac{n-1}{2}} d^{\times} x \\
&=\chi(-1)^{n-1} \gamma(1-s, \pi \otimes \chi, \psi) \int_{\mathbb{F}^{\times}} w(x) \chi(x)\|x\|^{1-s-\frac{n-1}{2}} d^{\times} x,
\end{aligned}
$$

for all $s$ of real part sufficiently large and all unitary multiplicative characters $\chi$ of $\mathbb{F}^{\times}$. (17.1) is independent of the chosen Haar measure $d^{\times} x$ on $\mathbb{F}^{\times}$, and uniquely defines $\widetilde{w}$ in terms of $\pi, \psi$ and $w$. We shall let the Haar measure be given as in $\$ 1.2$. We call $\widetilde{w}$ the Hankel transform of $w$ associated with $\pi$.

\footnotetext{
${ }^{\mathrm{I}}$ For a non-Archimedean local field, Hankel transforms can also be constructed in the same way.

${ }^{\mathrm{II}}$ Since $\pi$ is a local component of an irreducible cuspidal automorphic representation in [IT], [IT] also assumes that $\pi$ is unitary. However, if one only considers the local theory, this assumption is not necessary.
} 
According to [IT, Lemma 5.1], there exists a smooth Whitaker function $W \in \mathscr{W}(\pi, \psi)$ so that

$$
w(x)=W\left(\begin{array}{ll}
x & \\
& I_{n-1}
\end{array}\right),
$$

for all $x \in \mathbb{F}^{\times}$. Denote by $w_{n}$ the $n$-by- $n$ permutation matrix whose anti-diagonal entries are 1 , that is, the longest Weyl element of rank $n$, and define

$$
w_{n, 1}=\left(\begin{array}{cc}
1 & \\
& w_{n-1}
\end{array}\right) \text {. }
$$

In the theory of integral representations of Rankin-Selberg $L$-functions, (17.1) amounts to the local functional equations of zeta integrals for $\pi \otimes \chi$, with

$$
\widetilde{w}(x)=\widetilde{W}\left(\begin{array}{ll}
x & \\
& 1
\end{array}\right)=W\left(w_{2}\left(\begin{array}{ll}
x^{-1} & \\
& 1
\end{array}\right)\right),
$$

if $n=2$, and

$$
\widetilde{w}(x)=\int_{\mathbb{F}^{n-2}} \widetilde{W}\left(\left(\begin{array}{lll}
x & & \\
y & I_{n-2} & \\
& & 1
\end{array}\right) w_{n, 1}\right) d y_{\psi},
$$

if $n \geqslant 3$, where $\widetilde{W} \in \mathscr{W}\left(\tilde{\pi}, \psi^{-1}\right)$ is the dual Whittaker function defined by $\widetilde{W}(g)=$ $W\left(w_{n} \cdot{ }^{t} g^{-1}\right)$, for $g \in \mathrm{GL}_{n}(\mathbb{F})$, and $d x_{\psi}$ denotes the self-dual additive Haar measure on $\mathbb{F}$ with respect to $\psi$. See [IT Lemma 2.3].

It should be noted that the settings in [IT] can be extended in two aspects.

First, the constraint that $\pi$ be infinite dimensional and generic is actually dispensable for defining the Hankel transform via (17.1). In the following, we shall assume that $\pi$ is any irreducible admissible representation of $\mathrm{GL}_{n}(\mathbb{F})$. Moreover, we shall also include the case $n=1$. It will be seen that, after renormalizing the functions $w$ and $\widetilde{w}$, the Hankel transform defined by (17.1) converts into the Hankel transform given by (3.32) or (3.41). For this, we shall apply the Langlands classification for irreducible admissible representations of $\mathrm{GL}_{n}(\mathbb{F})$.

Second, the constraint that the weight function $w$ be compactly supported is not necessary. By the work in $\$ 2$ and 3 , the Hankel transform defined by (17.1) may be extended to a larger space of weight functions. Particularly important is that this space contains the Kirillov model when $n=2$ (see Remark 17.6).

17.1. Hankel Transforms over $\mathbb{R}$. Suppose $\mathbb{F}=\mathbb{R}$. Recall that \|\|$_{\mathbb{R}}=||$ is the ordinary absolute value. For $r \in \mathbb{R}^{\times}$let $\psi(x)=\psi_{r}(x)=e(r x)$.

According to [Kna §3, Lemma], every finite dimensional semisimple representation $\varphi$ of the Weil group of $\mathbb{R}$ may be decomposed into irreducible representations of dimension one or two. The one-dimensional representations are parametrized by $(\mu, \delta) \in \mathbb{C} \times \mathbb{Z} / 2 \mathbb{Z}$. We denote by $\varphi_{(\mu, \delta)}$ the representation given by $(\mu, \delta) . \varphi_{(\mu, \delta)}$ corresponds to the representation $\chi_{(\mu, \delta)}=\omega_{\mu} \eta^{\delta}$ of $\mathrm{GL}_{1}(\mathbb{R})$ under the Langlands correspondence over $\mathbb{R}$. The irreducible two-dimensional representations are parametrized by $(\mu, m) \in \mathbb{C} \times \mathbb{N}_{+}$. We denote by $\varphi_{(\mu, m)}$ the representation given by $(\mu, m) . \varphi_{(\mu, m)}$ corresponds to the representation $\sigma(m) \otimes \omega_{\mu}(\mathrm{det})$ of $\mathrm{GL}_{2}(\mathbb{R})$, where $\sigma(m)$ denotes the discrete series representation of weight $m$. 
In view of the formulae [Kna $(3.6,3.7)]^{[111}$ of $L$-functions and $\epsilon$-factors, the definitions of $G_{\delta}$ and $G_{m}$ in (1.3) and (1.6), along with the formula (1.10), we deduce that

$$
\gamma\left(s, \varphi_{(\mu, \delta)}, \psi\right)=\operatorname{sgn}(r)^{\delta}|r|^{s+\mu-\frac{1}{2}} G_{\delta}(1-s-\mu),
$$

whereas

$$
\gamma\left(s, \varphi_{(\mu, m)}, \psi\right)=\operatorname{sgn}(r)^{\delta(m)+1}|r|^{2 s+2 \mu-1} i G_{m}(1-s-\mu),
$$

and

$$
\begin{aligned}
\gamma\left(s, \varphi_{(\mu, m)}, \psi\right) & =\gamma\left(s, \varphi_{\left(\mu+\frac{1}{2} m, \delta(m)+1\right)}, \psi\right) \gamma\left(s, \varphi_{\left(\mu-\frac{1}{2} m, 0\right)}, \psi\right) \\
& =\gamma\left(s, \varphi_{\left(\mu+\frac{1}{2} m, \delta(m)\right)}, \psi\right) \gamma\left(s, \varphi_{\left(\mu-\frac{1}{2} m, 1\right)}, \psi\right) .
\end{aligned}
$$

To $\varphi_{(\mu, m)}$ we shall attach either one of the following two parameters

$$
\left(\mu+\frac{1}{2} m, \mu-\frac{1}{2} m, \delta(m)+1,0\right),\left(\mu+\frac{1}{2} m, \mu-\frac{1}{2} m, \delta(m), 1\right) .
$$

REMARK 17.1. 17.7) reflects the isomorphism $\varphi_{(0, m)} \otimes \varphi_{(0,1)} \cong \varphi_{(0, m)}$ of representations of the Weil group (here $(0,1)$ is an element in $\mathbb{C} \times \mathbb{Z} / 2 \mathbb{Z}$ ), as well as the isomorphism $\sigma(m) \otimes \eta \cong \sigma(m)$ of representations of $\mathrm{GL}_{2}(\mathbb{R})$.

For $\varphi$ reducible, $\gamma(s, \varphi, \psi)$ is the product of the $\gamma$-factors of the irreducible constituents of $\varphi$. Suppose that $\varphi$ is $n$-dimensional. It follows from (17.5, 17.6, 17.7) that there is a parameter $(\boldsymbol{\mu}, \boldsymbol{\delta}) \in \mathbb{C}^{n} \times(\mathbb{Z} / 2 \mathbb{Z})^{n}$ attached to $\varphi$ such that

$$
\gamma(s, \varphi, \psi)=\operatorname{sgn}(r)^{|\delta|}|r|^{n\left(s-\frac{1}{2}\right)+|\mu|} G_{(\mu, \delta)}(1-s) .
$$

The irreducible constituents of $\varphi$ are unique up to permutation, but, in view of the two different parameters attached to $\varphi_{(\mu, m)}$ in $(17.8)$, the parameter $(\boldsymbol{\mu}, \boldsymbol{\delta})$ attached to $\varphi$ may not.

Suppose that $\pi$ corresponds to $\varphi$ under the Langlands correspondence over $\mathbb{R}$. We have $\gamma(s, \pi, \psi)=\gamma(s, \varphi, \psi)$. It is known that $\pi$ is an irreducible constituent of the principal series representation unitarily induced from the character $\otimes_{l=1}^{n} \chi_{\left(\mu_{l}, \delta_{l}\right)}$ of the Borel subgroup. In particular,

$$
\omega_{\pi}(x)=\omega_{|\mu|}(x) \eta^{|\delta|}(x)=\operatorname{sgn}(x)^{|\delta|}|x|^{|\mu|} .
$$

Now let $\chi=\chi_{(0, \delta)}=\eta^{\delta}$ in 17.1 , $\delta \in \mathbb{Z} / 2 \mathbb{Z}$. In view of [17.9) and (17.10), one has the following expression of the $\gamma$-factor in (17.1),

$$
\gamma\left(1-s, \pi \otimes \eta^{\delta}, \psi\right)=\omega_{\pi}(r)\left(\operatorname{sgn}(r)^{\delta}|r|^{\frac{1}{2}-s}\right)^{n} G_{\left(\mu, \delta+\delta \boldsymbol{e}^{n}\right)}(s) .
$$

Some calculations show that (17.1) is exactly translated into 3.32$)$ if one lets

$$
\begin{aligned}
v(x) & =\omega_{\pi}(r) w\left(|r|^{-\frac{n}{2}} x\right)|x|^{-\frac{n-1}{2}}, \\
\Upsilon(x) & =\widetilde{w}\left((-)^{n-1} \operatorname{sgn}(r)^{n}|r|^{-\frac{n}{2}} x\right)|x|^{-\frac{n-1}{2}} .
\end{aligned}
$$

Then, (3.37) can be reformulated as

$$
\widetilde{w}\left((-)^{n-1} x\right)=\omega_{\pi}(r)|r|^{\frac{n}{2}}|x|^{\frac{n-1}{2}} \int_{\mathbb{R}^{\times}} w(y) J_{(\mu, \delta)}\left(r^{n} x y\right)|y|^{1-\frac{n-1}{2}} d^{\times} y .
$$

\footnotetext{
${ }^{\mathrm{III}}$ The formulae in $\left.\mathbf{K n a}(3.6,3.7)\right]$ are for $\psi_{1}$. The relation between the epsilon factors $\epsilon\left(s, \pi, \psi_{r}\right)$ and $\epsilon(s, \pi, \psi)$ is given in [Tat \$3] (see in particular [Tat (3.6.6)]).
} 
17.2. Hankel Transforms over $\mathbb{C}$. Suppose $\mathbb{F}=\mathbb{C}$. Recall that \|\|$_{\mathbb{C}}=\|\|=||^{2}$, where || denotes the ordinary absolute value. For $r \in \mathbb{C}^{\times}$let $\psi(z)=\psi_{r}(z)=e(r z+\overline{r z})$.

The Langlands classification and correspondence for $\mathrm{GL}_{n}(\mathbb{C})$ are less complicated. First of all, the Weil group of $\mathbb{C}$ is simply $\mathbb{C}^{\times}$. Any $n$-dimensional semisimple representation $\varphi$ of the Weil group $\mathbb{C}^{\times}$is the direct sum of one-dimensional representations. The one-dimensional representations are of the form $\chi_{(\mu, m)}=\omega_{\mu} \eta^{m}$, with $(\mu, m) \in \mathbb{C} \times \mathbb{Z}$. In view of the formulae [Kna $(4.6,4.7)]$ of $L\left(s, \chi_{(\mu, m)}\right)$ and $\epsilon\left(s, \chi_{(\mu, m)}, \psi\right)$ as well as the definition of $G_{m}$ in $(1.6)$, we have

$$
\gamma\left(s, \chi_{(\mu, m)}, \psi\right)=[r]^{m}\|r\|^{s+\mu-\frac{1}{2}} G_{m}(1-s-\mu) .
$$

Thus $\varphi$ is parametrized by some $(\boldsymbol{\mu}, \boldsymbol{m}) \in \mathbb{C}^{n} \times \mathbb{Z}^{n}$ and

$$
\gamma(s, \varphi, \psi)=[r]^{|\boldsymbol{m}|}\|r\|^{n\left(s-\frac{1}{2}\right)+|\boldsymbol{\mu}|} G_{(\boldsymbol{\mu}, \boldsymbol{m})}(1-s) .
$$

This parametrization is unique up to permutation, in contrast to the case $\mathbb{F}=\mathbb{R}$.

If $\pi$ corresponds to $\varphi$ under the Langlands correspondence over $\mathbb{C}$, then one has $\gamma(s, \pi, \psi)=\gamma(s, \varphi, \psi)$. Moreover, $\pi$ is an irreducible constituent of the principal series representation unitarily induced from the character $\bigotimes_{l=1}^{n} \chi_{\left(\mu_{l}, m_{l}\right)}$ of the Borel subgroup. Note that

$$
\omega_{\pi}(z)=\omega_{|\boldsymbol{\mu}|}(z) \eta^{|\boldsymbol{m}|}(z)=[z]^{|\boldsymbol{m}|}\|z\|^{|\boldsymbol{\mu}|} .
$$

Now let $\chi=\chi_{(0, m)}=\eta^{m}$ in $17.1, m \in \mathbb{Z}$. Then 17.15 and 17.16 imply

$$
\gamma\left(1-s, \pi \otimes \eta^{m}, \psi\right)=\omega_{\pi}(r)\left([r]^{m}\|r\|^{\frac{1}{2}-s}\right)^{n} G_{\left(\mu, \boldsymbol{m}+m \boldsymbol{e}^{n}\right)}(s) .
$$

By putting

$$
\begin{aligned}
v(z) & =\omega_{\pi}(r) w\left(\|r\|^{-\frac{n}{2}} z\right)\|z\|^{-\frac{n-1}{2}}, \\
\Upsilon(z) & =\widetilde{w}\left((-)^{n-1}[r]^{-n}\|r\|^{-\frac{n}{2}} z\right)\|z\|^{-\frac{n-1}{2}},
\end{aligned}
$$

the identity (17.1) is translated into (3.41), and (3.47) can be reformulated as

$$
\widetilde{w}\left((-)^{n-1} z\right)=\omega_{\pi}(r)\|r\|^{\frac{n}{2}}\|z\|^{\frac{n-1}{2}} \int_{\mathbb{C}^{\times}} w(u) J_{(\boldsymbol{\mu}, \boldsymbol{m})}\left(r^{n} z u\right)\|u\|^{1-\frac{n-1}{2}} d^{\times} u .
$$

17.3. Some New Notations. Let $\pi$ be an irreducible admissible representation of $\mathrm{GL}_{n}(\mathbb{F})$. For $\mathbb{F}=\mathbb{R}$, respectively $\mathbb{F}=\mathbb{C}$, if $\pi$ is parametrized by $(\boldsymbol{\mu}, \boldsymbol{\delta})$, respectively $(\boldsymbol{\mu}, \boldsymbol{m})$, we shall denote by $J_{\pi}$ the Bessel kernel $J_{(\boldsymbol{\mu}, \boldsymbol{\delta})}$, respectively $J_{(\boldsymbol{\mu}, \boldsymbol{m})}$. Thus, 17.13) and $(17.19)$ can be uniformly combined into one formula

$$
\widetilde{w}\left((-)^{n-1} x\right)=\omega_{\pi}(r)\|r\|^{\frac{n}{2}}\|x\|^{\frac{n-1}{2}} \int_{\mathbb{F}^{\times}} w(y) J_{\pi}\left(r^{n} x y\right)\|y\|^{1-\frac{n-1}{2}} d^{\times} y .
$$

Proposition 3.14 (1) and 3.17(1) are translated into the following lemma.

Lemмa 17.2. Let $\pi$ be an irreducible admissible representation of $\mathrm{GL}_{n}(\mathbb{F})$, and let $\chi$ be a character on $\mathbb{F}^{\times}$. We have $J_{\chi \otimes \pi}(x)=\chi^{-1}(x) J_{\pi}(x)$.

REMARK 17.3. Let $\mathrm{Z}_{n}$ denote the center of $\mathrm{GL}_{n}$. In view of Lemma 17.2, no generality will be lost if one only considers $J_{\pi}$ for irreducible admissible representations $\pi$ of $\mathrm{GL}_{n}(\mathbb{F}) / \mathrm{Z}_{n}\left(\mathbb{R}_{+}\right)$.

Let $\varphi$ be the $n$-dimensional semisimple representation of the Weil group of $\mathbb{F}$ corresponding to $\pi$ under the Langlands correspondence over $\mathbb{F}$.

When $\mathbb{F}=\mathbb{R}$, the function space $\mathscr{S}_{\text {sis }}^{(\mu, \delta)}\left(\mathbb{R}^{\times}\right)$depends on the choice of the parameter $(\boldsymbol{\mu}, \boldsymbol{\delta})$ attached to $\varphi$, if some discrete series $\varphi_{(\mu, m)}$ occurs in its decomposition. Thus one 
needs to redefine the function spaces for Hankel transforms according to the Langlands classification rather than the above parametrization. For this, let $n_{1}, n_{2} \in \mathbb{N},\left(\boldsymbol{\mu}^{1}, \boldsymbol{\delta}^{1}\right) \in$ $\mathbb{C}^{n_{1}} \times(\mathbb{Z} / 2 \mathbb{Z})^{n_{1}}$ and $\left(\boldsymbol{\mu}^{2}, \boldsymbol{m}^{2}\right) \in \mathbb{C}^{n_{2}} \times \mathbb{N}_{+}^{n_{2}}$ be such that $n_{1}+2 n_{2}=n$ and $\varphi=\bigoplus_{l=1}^{n_{1}} \varphi_{\left(\mu_{l}^{1}, \delta_{l}^{1}\right)} \oplus$ $\bigoplus_{l=1}^{n_{2}} \varphi_{\left(\mu_{l}^{2}, m_{l}^{2}\right)}$. We define the function space $\mathscr{S}_{\text {sis }}^{\pi}\left(\mathbb{R}^{\times}\right)=\mathscr{S}_{\text {sis }}^{\varphi}\left(\mathbb{R}^{\times}\right)$to be

$$
\mathscr{S}_{\text {sis }}^{\left(-\boldsymbol{\mu}^{1}, \boldsymbol{\delta}^{1}\right)}\left(\mathbb{R}^{\times}\right)+\mathscr{S}_{\text {sis }}^{\left(-\boldsymbol{\mu}^{2}, \boldsymbol{m}^{2}\right)}\left(\mathbb{R}^{\times}\right),
$$

where $\mathscr{S}_{\text {sis }}^{(\mu, \delta)}\left(\mathbb{R}^{\times}\right)$is defined by 3.30 , and

$$
\mathscr{S}_{\text {sis }}^{(\boldsymbol{\mu}, \boldsymbol{m})}\left(\mathbb{R}^{\times}\right)=\sum_{\delta \in \mathbb{Z} / 2 \mathbb{Z}} \operatorname{sgn}(x)^{\delta} \mathscr{S}_{\text {sis }}^{\boldsymbol{\mu}-\frac{1}{2} \boldsymbol{m}}\left(\mathbb{R}_{+}\right),
$$

with $\mathscr{S}_{\text {sis }}^{\lambda}\left(\mathbb{R}_{+}\right)$defined by 3.2 .

Lemma 17.4. Let $(\boldsymbol{\mu}, \boldsymbol{m}) \in \mathbb{C}^{n} \times \mathbb{N}_{+}^{n}$. Suppose $v \in \mathscr{S}_{\text {sis }}^{(-\mu, \boldsymbol{m})}\left(\mathbb{R}^{\times}\right)$. Then there exists a unique function $\Upsilon \in \mathscr{S}_{\text {sis }}^{(\mu, m)}\left(\mathbb{R}^{\times}\right)$satisfying the following two identities,

$$
\mathcal{M}_{\delta} \Upsilon(s)=i^{n} G_{(\mu, \boldsymbol{m})}(s) \mathcal{M}_{\delta} v(1-s), \quad \delta \in \mathbb{Z} / 2 \mathbb{Z}
$$

Writing $\mathcal{H}_{(\mu, \boldsymbol{m})}^{2} v=\Upsilon$, we have

$$
\mathcal{H}_{(\boldsymbol{\mu}, \boldsymbol{m})}^{2} v(x)=\Upsilon(x), \quad \mathcal{H}_{(-\boldsymbol{\mu}, \boldsymbol{m})}^{2} \Upsilon(x)=(-)^{|\boldsymbol{m}|+n} v(x) .
$$

When $\mathbb{F}=\mathbb{C}$, we put

$$
\mathscr{S}_{\text {sis }}^{\pi}\left(\mathbb{C}^{\times}\right)=\mathscr{S}_{\text {sis }}^{\varphi}\left(\mathbb{C}^{\times}\right)=\mathscr{S}_{\text {sis }}^{(-\mu,-m)}\left(\mathbb{C}^{\times}\right) .
$$

Let $d=[\mathbb{F}: \mathbb{R}]$. For each character $\chi$ on $\mathbb{F}^{\times} / \mathbb{R}_{+}$we define the Mellin transform $\mathcal{M}_{\chi}$ of a function $v \in \mathscr{S}_{\text {sis }}\left(\mathbb{F}^{\times}\right)$by

$$
\mathcal{M}_{\chi} v(s)=\int_{\mathbb{F}^{\times}} v(x) \chi(x)\|x\|^{\frac{1}{d} s} d^{\times} x .
$$

THEOREM 17.5. Let $\pi$ be an irreducible admissible representation of $\mathrm{GL}_{n}(\mathbb{F})$. Suppose $v \in \mathscr{S}_{\text {sis }}^{\pi}\left(\mathbb{F}^{\times}\right)$. Then there exists a unique $\widetilde{v} \in \mathscr{S}_{\text {sis }}^{\tilde{\pi}}\left(\mathbb{F}^{\times}\right)$satisfying the following identity

$$
\mathcal{M}_{\chi^{-1}} \widetilde{v}(d s)=\gamma\left(1-s, \pi \otimes \chi, \psi_{1}\right) \mathcal{M}_{\chi} v(d(1-s))
$$

for all characters $\chi$ on $\mathbb{F}^{\times} / \mathbb{R}_{+}$. We write $\mathcal{H}_{\pi} v=\tilde{v}$ and call $\tilde{v}$ the normalized Hankel transform of $v$ over $\mathbb{F}^{\times}$associated with $\pi$. Moreover, we have the Hankel inversion formula

$$
\mathcal{H}_{\pi} v(x)=\widetilde{v}(x), \quad \mathcal{H}_{\tilde{\pi}} \widetilde{v}(x)=\omega_{\pi}(-1) v\left((-)^{n} x\right) .
$$

Proof. If $\mathbb{F}=\mathbb{R}$, this follows from Theorem 3.12 and Lemma 17.4 If $\mathbb{F}=\mathbb{C}$, this is simply a translation of Theorem 3.15

Q.E.D.

Remark 17.6. Now let $n=2$. In view of [JL, §5, 6] or [God §2.5], it may be checked that $\|x\|^{-\frac{1}{2}} w(x)$ lies in the space $\mathscr{S}_{\text {sis }}^{\pi}\left(\mathbb{F}^{\times}\right)$if $w$ is a function in the Kirillov model of $\pi$, namely,

$$
\left\{\|x\|^{-\frac{1}{2}} W\left(\begin{array}{ll}
x & \\
& 1
\end{array}\right): W \in \mathscr{W}\left(\pi, \psi_{1}\right)\right\} \subset \mathscr{S}_{\text {sis }}^{\pi}\left(\mathbb{F}^{\times}\right) .
$$

Moreover, it follows from the formula of $J_{\pi}$ in $\$ 4.3$ and $\$ 15.3$ that the integral in (17.20) is absolutely convergent and (17.20) is valid for all $\|x\|^{-\frac{1}{2}} w(x) \in \mathscr{S}_{\text {sis }}^{\pi}\left(\mathbb{F}^{\times}\right)$if $\pi$ is either a principal series representation with parameter $|\mathfrak{R e} \mu|<\frac{1}{2}$ or a discrete series representation. For the principal series case, see also Proposition 3.14 (3) and 3.17(3). In particular, if $\pi$ is unitary then (17.20) is valid for all $w$ in the Kirillov model of $\pi$. 


\section{Bessel Functions for $\mathrm{GL}_{2}(\mathbb{F})$}

Let $n=2$ and retain the notations from $\$ 17$ except for the different choice of the Weyl element $w_{2}=\left(\begin{array}{ll}1 & -1 \\ 1 & \end{array}\right)$, which is more often used for $\mathrm{GL}_{2}$ in the literature.

Let $\pi$ be an infinite dimensional irreducible unitary representation of $\left.\mathrm{GL}_{2}(\mathbb{F})\right]^{\mathrm{IV}}$ Using 17.2, 17.3 and Remark 17.6, one may rewrite 17.20 as follows,

$$
W\left(\begin{array}{cc} 
& 1 \\
-x^{-1} &
\end{array}\right)=\omega_{\pi}(r)\|r\| \int_{\mathbb{F}^{\times}}\|x y\|^{\frac{1}{2}} J_{\pi}\left(r^{2} x y\right) W\left(\begin{array}{ll}
y & \\
& 1
\end{array}\right) d^{\times} y,
$$

for all $W \in \mathscr{W}\left(\pi, \psi_{r}\right)$. We define

$$
\mathcal{\partial}_{\pi, \psi_{r}}(x)=\omega_{\pi}(-r x)\|r\| \sqrt{\|x\|} J_{\pi}\left(r^{2} x\right) .
$$

We call $\mathcal{J}_{\pi, \psi}(x)$ the Bessel function associated with $\pi$ and $\psi$. The formula 18.1) then reads

$$
\omega_{\pi}(-x) W\left(\begin{array}{cc} 
& 1 \\
-x^{-1} &
\end{array}\right)=\int_{\mathbb{F}^{\times}} \partial_{\pi, \psi}(x y) \omega_{\pi}(y)^{-1} W\left(\begin{array}{ll}
y & \\
& 1
\end{array}\right) d^{\times} y .
$$

Moreover, with the observation

$$
\omega_{\pi}(-x) W\left(\begin{array}{ll} 
& 1 \\
-x^{-1} &
\end{array}\right)=W\left(\left(\begin{array}{ll}
x & \\
& 1
\end{array}\right) w_{2}\right)
$$

(18.3) turns into

$$
W\left(\left(\begin{array}{ll}
x & \\
& 1
\end{array}\right) w_{2}\right)=\int_{\mathbb{F}^{\times}} \omega_{\pi}(y)^{-1} \mathcal{\partial}_{\pi, \psi}(x y) W\left(\begin{array}{ll}
y & \\
& 1
\end{array}\right) d^{\times} y \mathrm{v}
$$

Thus (18.4) indicates that the action of the Weyl element $w_{2}$ on the Kirillov model

$$
\mathscr{K}(\pi, \psi)=\left\{w(x)=W\left(\begin{array}{ll}
x & \\
& 1
\end{array}\right): W \in \mathscr{W}(\pi, \psi)\right\}
$$

is essentially a Hankel transform. From this perspective, the Hankel inversion formula follows from the simple identity $w_{2}^{2}=-I_{2}$. This may be seen from the following lemma.

Lemma 18.1. Let $\pi$ be an irreducible admissible representation of $\mathrm{GL}_{2}(\mathbb{F})$. Then we have $J_{\tilde{\pi}}(x)=\omega_{\pi}(x) J_{\pi}(x)$.

Proof. This follows from some straightforward calculations using Proposition 3.14 (1) and 3.17 (1).

Q.E.D.

Remark 18.2. The representation theoretic viewpoint of Lemma 18.1 is the isomorphism $\tilde{\pi} \cong \omega^{-1} \otimes \pi$. With this, Lemma 18.1 is a direct consequence of Lemma 17.2.

Finally, we shall summarize the formulae of the Bessel functions associated with infinite dimensional irreducible unitary representations of $\mathrm{GL}_{2}(\mathbb{F})$. First of all, in view of Lemma 17.2 and Remark 17.3. one may assume without loss of generality that $\pi$ is trivial on $\mathrm{Z}_{2}\left(\mathbb{R}_{+}\right)$. Moreover, with the simple observation

$$
\mathcal{J}_{\pi, \psi_{r}}(x)=\omega_{\pi}(r)^{-1} \mathcal{J}_{\pi, \psi_{1}}\left(r^{2} x\right),
$$

it is sufficient to consider the Bessel function $\mathcal{J}_{\pi}=\mathcal{J}_{\pi, \psi_{1}}$ associated with $\psi_{1}$.

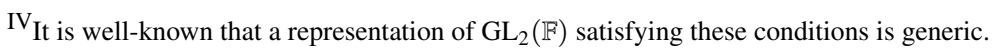

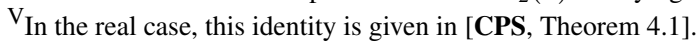


18.1. Bessel Functions for $\mathrm{GL}_{2}(\mathbb{R})$. Under the Langlands correspondence, we have the following classification of infinite dimensional irreducible unitary representations of $\mathrm{GL}_{2}(\mathbb{R}) / \mathrm{Z}_{2}\left(\mathbb{R}_{+}\right)$.

- (principal series and the limit of discrete series) $\varphi_{(i t, \epsilon+\delta)} \oplus \varphi_{(-i t, \epsilon)}$, with $t \in \mathbb{R}$ and $\epsilon, \delta \in \mathbb{Z} / 2 \mathbb{Z}$,

- (complementary series) $\varphi_{(t, \epsilon)} \oplus \varphi_{(-t, \epsilon)}$, with $t \in\left(0, \frac{1}{2}\right)$ and $\epsilon \in \mathbb{Z} / 2 \mathbb{Z}$,

- (discrete series) $\varphi_{(0, m)}$, with $m \in \mathbb{N}_{+}$.

Here, in the first case, the corresponding representation is a limit of discrete series if $t=0$ and $\delta=1$ and a principal series representation if otherwise. We shall write the corresponding representations as $\eta^{\epsilon} \otimes \pi^{+}(i t)$ if $\delta=0, \eta^{\epsilon} \otimes \pi^{-}(i t)$ if $\delta=1, \eta^{\epsilon} \otimes \pi(t)$ and $\sigma(m)$, respectively. We have

$$
\omega_{\pi^{+}(i t)}=1, \quad \omega_{\pi^{-}(i t)}=\eta, \quad \omega_{\pi(t)}=1, \quad \omega_{\sigma(m)}=\eta^{m+1} .
$$

Furthermore, we have the equivalences $\pi^{+}(i t) \cong \pi^{+}(-i t)$ and $\pi^{-}(i t) \cong \eta \otimes \pi^{-}(-i t)$.

According to $\$ 4.3$, we have the following proposition.

Proposition 18.3.

(1). Let $t \in \mathbb{R}$. We have for $x \in \mathbb{R}_{+}$

$$
\begin{aligned}
\mathcal{J}_{\pi^{+}(i t)}(x) & =\frac{\pi i}{\sinh (\pi t)} \sqrt{x}\left(J_{2 i t}(4 \pi \sqrt{x})-J_{-2 i t}(4 \pi \sqrt{x})\right), \\
\mathcal{J}_{\pi^{+}(i t)}(-x) & =4 \cosh (\pi t) \sqrt{x} K_{2 i t}(4 \pi \sqrt{x}),
\end{aligned}
$$

where it is understood that when $t=0$ the right hand side of the first formula should be replaced by its limit, and

$$
\begin{aligned}
\mathcal{J}_{\pi^{-}(i t)}(x) & =-\frac{\pi i}{\cosh (\pi t)} \sqrt{x}\left(J_{2 i t}(4 \pi \sqrt{x})+J_{-2 i t}(4 \pi \sqrt{x})\right), \\
\partial_{\pi^{-}(i t)}(-x) & =4 \sinh (\pi t) \sqrt{x} K_{2 i t}(4 \pi \sqrt{x}) .
\end{aligned}
$$

(2). Let $t \in\left(0, \frac{1}{2}\right)$. We have for $x \in \mathbb{R}_{+}$

$$
\begin{aligned}
\mathcal{J}_{\pi(t)}(x) & =-\frac{\pi}{\sin (\pi t)} \sqrt{x}\left(J_{2 t}(4 \pi \sqrt{x})-J_{-2 t}(4 \pi \sqrt{x})\right), \\
\mathcal{J}_{\pi(t)}(-x) & =4 \cos (\pi t) \sqrt{x} K_{2 t}(4 \pi \sqrt{x}) .
\end{aligned}
$$

(3). Let $m \in \mathbb{N}_{+}$. We have for $x \in \mathbb{R}_{+}$

$$
\mathcal{J}_{\sigma(m)}(x)=2 \pi(-i)^{m+1} \sqrt{x} J_{m}(4 \pi \sqrt{x}), \quad \mathcal{J}_{\sigma(m)}(-x)=0 .
$$

Remark 18.4. $\eta^{\epsilon} \otimes \pi^{+}(i t), \eta^{\epsilon} \otimes \pi(t)$ and $\sigma(2 d-1)$ exhaust all the infinite dimensional irreducible unitary representations of $\mathrm{PGL}_{2}(\mathbb{R})$. Their Bessel functions are also given in [CPS, Proposition 6.1].

18.2. Bessel Functions for $\mathrm{GL}_{2}(\mathbb{C})$. Under the Langlands correspondence, we have the following classification of infinite dimensional irreducible unitary representations of $\mathrm{GL}_{2}(\mathbb{C}) / \mathrm{Z}_{2}\left(\mathbb{R}_{+}\right)$.

- (principal series) $\chi_{(i t, k+d+\delta)} \oplus \chi_{(-i t, k-d)}$, with $t \in \mathbb{R}, k, d \in \mathbb{Z}$ and $\delta \in \mathbb{Z} / 2 \mathbb{Z}=$ $\{0,1\}$,

- (complementary series) $\chi_{(t, k)} \oplus \chi_{(-t, k)}$, with $t \in\left(0, \frac{1}{2}\right)$ and $k \in \mathbb{Z}$.

We write the corresponding representations as $\eta^{k} \otimes \pi_{d}^{+}(i t)$ if $\delta=0, \eta^{k} \otimes \pi_{d}^{-}(i t)$ if $\delta=1$ and $\eta^{k} \otimes \pi(t)$, respectively. We have

$$
\omega_{\pi_{d}^{+}(i t)}=1, \quad \omega_{\pi_{d}^{-}(i t)}=\eta, \quad \omega_{\pi(t)}=1 .
$$


Furthermore, we have the equivalences $\pi_{d}^{+}(i t) \cong \pi_{-d}^{+}(-i t), \pi_{d}^{-}(i t) \cong \pi_{-d-1}^{-}(-i t)$.

According to Example 15.6, we have the following proposition.

Proposition 18.5. Recall the definitions 15.13.15.16 of $J_{\mu, m}(z)$ and $H_{\mu, m}^{(1,2)}(z)$ in Example 15.6

(1). Let $t \in \mathbb{R}$ and $d \in \mathbb{Z}$. We have for $z \in \mathbb{C}^{\times}$

$$
\begin{aligned}
\partial_{\pi_{d}^{+}(i t)}(z) & =-\frac{2 \pi^{2} i}{\sinh (2 \pi t)}|z|\left(J_{i t, 2 d}(4 \pi \sqrt{z})-J_{-i t,-2 d}(4 \pi \sqrt{z})\right) \\
& =\pi^{2} i|z|\left(e^{-2 \pi t} H_{i t, 2 d}^{(1)}(4 \pi \sqrt{z})-e^{2 \pi t} H_{i t, 2 d}^{(2)}(4 \pi \sqrt{z})\right), \\
\partial_{\pi_{d}^{-}(i t)}(z) & =-\frac{2 \pi^{2} i}{\cosh (2 \pi t)} \sqrt{|z| z}\left(J_{i t, 2 d+1}(4 \pi \sqrt{z})+J_{-i t,-2 d-1}(4 \pi \sqrt{z})\right) \\
& =-\pi^{2} i \sqrt{|z| z}\left(e^{-2 \pi t} H_{i t, 2 d+1}^{(1)}(4 \pi \sqrt{z})+e^{2 \pi t} H_{i t, 2 d+1}^{(2)}(4 \pi \sqrt{z})\right) .
\end{aligned}
$$

(2). Let $t \in\left(0, \frac{1}{2}\right)$. We have for $z \in \mathbb{C}^{\times}$

$$
\begin{aligned}
\partial_{\pi(t)}(z) & =\frac{2 \pi^{2}}{\sin (2 \pi t)}|z|\left(J_{t, 0}(4 \pi \sqrt{z})-J_{-t, 0}(4 \pi \sqrt{z})\right) \\
& =\pi^{2} i|z|\left(e^{2 \pi i t} H_{t, 0}^{(1)}(4 \pi \sqrt{z})-e^{-2 \pi i t} H_{t, 0}^{(2)}(4 \pi \sqrt{z})\right) .
\end{aligned}
$$

In view of Corollary 6.17, we have the following integral representations of $\mathcal{J}_{\pi}\left(x e^{i \phi}\right)$ except for $\pi=\pi(t)$ and $t \in\left[\frac{3}{8}, \frac{1}{2}\right)$.

Proposition 18.6.

(1). Let $t \in \mathbb{R}$ and $d \in \mathbb{Z}$. We have for $x \in \mathbb{R}_{+}$and $\phi \in \mathbb{R} / 2 \pi \mathbb{Z}$

$$
\begin{aligned}
& \partial_{\pi_{d}^{+}(i t)}\left(x e^{i \phi}\right)=4 \pi x\left(-e^{i \phi}\right)^{d} \int_{0}^{\infty} y^{4 i t-1}\left[y^{-1}+y e^{i \phi}\right]^{-2 d} J_{2 d}\left(4 \pi \sqrt{x}\left|y^{-1}+y e^{i \phi}\right|\right) d y, \\
& \partial_{\pi_{d}^{-}(i t)}\left(x e^{i \phi}\right)=4 \pi i x\left(-e^{i \phi}\right)^{d+1} \int_{0}^{\infty} y^{4 i t-1}\left[y^{-1}+y e^{i \phi}\right]^{-2 d-1} J_{2 d+1}\left(4 \pi \sqrt{x}\left|y^{-1}+y e^{i \phi}\right|\right) d y .
\end{aligned}
$$

(2). Let $t \in\left(0, \frac{3}{8}\right)$. We have for $x \in \mathbb{R}_{+}$and $\phi \in \mathbb{R} / 2 \pi \mathbb{Z}$

$$
\partial_{\pi(t)}\left(x e^{i \phi}\right)=4 \pi x \int_{0}^{\infty} y^{4 t-1} J_{0}\left(4 \pi \sqrt{x}\left|y^{-1}+y e^{i \phi}\right|\right) d y,
$$

in which the integral converges absolutely only for $t \in\left(0, \frac{1}{8}\right)$.

REMARK 18.7. $\pi_{d}^{+}(i t)$ and $\pi(t)$ exhaust all the infinite dimensional irreducible unitary representations of $\mathrm{PGL}_{2}(\mathbb{C})$. Proposition 18.5 shows that the Bessel function for $\pi_{d}^{+}(i t)$ actually coincide with that given in [BM5]. More precisely, we have the equality $\mathcal{\partial}_{\pi_{d}^{+}(i t)}(z)=2 \pi^{2}|z| \mathcal{K}_{2 i t,-d}(4 \pi \sqrt{z})$, with $\mathcal{K}_{v, p}$ given by [BM5, (6.21), (7.21)]. Furthermore, the integral representation of $\mathcal{J}_{\pi_{d}^{+}(i t)}$ in Proposition 18.6 (1) is tantamount to [BM5, Theorem 12.1]. We have similar relations between the Bessel function for $\pi_{d}^{-}(i t)$ and that given in [LG]. 


\section{Bibliography}

[Blo] V. Blomer. Subconvexity for twisted L-functions on GL(3). Amer. J. Math., 134(5):1385-1421, 2012.

[BM1] E. M. Baruch and Z. Mao. Bessel identities in the Waldspurger correspondence over a $p$-adic field. Amer. J. Math., 125(2):225-288, 2003.

[BM2] E. M. Baruch and Z. Mao. Bessel identities in the Waldspurger correspondence over the real numbers. Israel J. Math., 145:1-81, 2005.

[BM3] E. M. Baruch and Z. Mao. A Whittaker-Plancherel inversion formula for $\mathrm{SL}(2, \mathbb{R})$. J. Funct. Anal., 238(1):221-244, 2006.

[BM4] E. M. Baruch and Z. Mao. Central value of automorphic L-functions. Geom. Funct. Anal., 17(2):333384, 2007.

[BM5] R. W. Bruggeman and Y. Motohashi. Sum formula for Kloosterman sums and fourth moment of the Dedekind zeta-function over the Gaussian number field. Funct. Approx. Comment. Math., 31:23-92, 2003.

[But] J. Buttcane. The spectral Kuznetsov formula on SL(3). Trans. Amer. Math. Soc., 368(9):6683-6714, 2016.

[CL] E. A. Coddington and N. Levinson. Theory of Ordinary Differential Equations. McGraw-Hill Book Company, Inc., New York-Toronto-London, 1955.

[Cog] J. W. Cogdell. Lectures on L-functions, converse theorems, and functoriality for $\mathrm{GL}_{n}$. Lectures on Automorphic L-Functions, Fields Inst. Monogr., vol. 20, pp. 1-96. Amer. Math. Soc., Providence, RI, 2004.

[CPS] J. W. Cogdell and I. Piatetski-Shapiro. The Arithmetic and Spectral Analysis of Poincaré Series. Perspectives in Mathematics, vol. 13. Academic Press, Inc., Boston, MA, 1990.

[EM] W. N. Everitt and C. Markett. On a generalization of Bessel functions satisfying higher-order differential equations. J. Comput. Appl. Math., 54(3):325-349, 1994.

[GL1] D. Goldfeld and X.Q. Li. Voronoi formulas on GL(n). Int. Math. Res. Not., pages Art. ID 86295, 25, 2006.

[GL2] D. Goldfeld and X.Q. Li. The Voronoi formula for GL(n, R). Int. Math. Res. Not., (2), pages Art. ID rnm144, 39, 2008.

[God] R. Godement. Notes on Jacquet-Langlands' Theory. The Institute for Advanced Study, Princeton, NJ, 1970.

[GR] I. S. Gradshteyn and I. M. Ryzhik. Table of Integrals, Series, and Products. Elsevier/Academic Press, Amsterdam, 7th ed., 2007.

[HM] G. Harcos and P. Michel. The subconvexity problem for Rankin-Selberg $L$-functions and equidistribution of Heegner points. II. Invent. Math., 163(3):581-655, 2006.

[Hör] L. Hörmander. The Analysis of Linear Partial Differential Operators. I. Grundlehren der Mathematischen Wissenschaften, vol. 256. Springer-Verlag, Berlin, 1983.

[IT] A. Ichino and N. Templier. On the Voronor formula for GL(n). Amer. J. Math., 135(1):65-101, 2013.

[Ivi] A. Ivić. On the ternary additive divisor problem and the sixth moment of the zeta-function. Sieve Methods, Exponential Sums, and Their Applications in Number Theory (Cardiff, 1995), London Math. Soc. Lecture Note Ser., vol. 237, pp. 205-243. Cambridge Univ. Press, Cambridge, 1997.

[JL] H. Jacquet and R. P. Langlands. Automorphic Forms on GL(2). Lecture Notes in Mathematics, Vol. 114. Springer-Verlag, Berlin-New York, 1970.

[KMV] E. Kowalski, P. Michel, and J. VanderKam. Rankin-Selberg L-functions in the level aspect. Duke Math. J., 114(1):123-191, 2002.

[Kna] A. W. Knapp. Local Langlands correspondence: the Archimedean case. Motives (Seattle, WA, 1991), Proc. Sympos. Pure Math., vol. 55, pp. 393-410. Amer. Math. Soc., Providence, RI, 1994.

[Kuz] N. V. Kuznetsov. Petersson's conjecture for cusp forms of weight zero and Linnik's conjecture. Sums of Kloosterman sums. Math. Sbornik, 39:299-342, 1981. 
[LG] H. Lokvenec-Guleska. Sum Formula for $\mathrm{SL}_{2}$ over Imaginary Quadratic Number Fields. Ph.D. Thesis. Utrecht University, 2004.

[Li1] X. Li. Bounds for GL(3) $\times$ GL(2) L-functions and GL(3) L-functions. Ann. of Math. (2), 173(1):301336, 2011.

[Li2] X.Q. Li. The central value of the Rankin-Selberg L-functions. Geom. Funct. Anal., 18(5):1660-1695, 2009.

[Mil] S. D. Miller. Cancellation in additively twisted sums on GL(n). Amer. J. Math., 128(3):699-729, 2006.

[MS1] S. D. Miller and W. Schmid. Distributions and analytic continuation of Dirichlet series. J. Funct. Anal., 214(1):155-220, 2004.

[MS2] S. D. Miller and W. Schmid. Summation formulas, from Poisson and Voronoi to the present. Noncommutative Harmonic Analysis, Progr. Math., vol. 220, pp. 419-440. Birkhäuser Boston, Boston, MA, 2004.

[MS3] S. D. Miller and W. Schmid. Automorphic distributions, $L$-functions, and Voronoi summation for GL(3). Ann. of Math. (2), 164(2):423-488, 2006.

[MS4] S. D. Miller and W. Schmid. A general Voronoi summation formula for $\operatorname{GL}(n, \mathbb{Z})$. Geometry and Analysis. No. 2, Adv. Lect. Math. (ALM), vol. 18, pp. 173-224. Int. Press, Somerville, MA, 2011.

[Mun] R. Munshi. Shifted convolution sums for GL(3) × GL(2). Duke Math. J., 162(13):2345-2362, 2013.

[Nar] R. Narasimhan. Analysis on Real and Complex Manifolds, North-Holland Mathematical Library, vol. 35. North-Holland Publishing Co., Amsterdam, 1985. Reprint of the 1973 edition.

[Olv1] F. W. J. Olver. The asymptotic expansion of Bessel functions of large order. Philos. Trans. Roy. Soc. London. Ser. A., 247:328-368, 1954.

[Olv2] F. W. J. Olver. The asymptotic solution of linear differential equations of the second order for large values of a parameter. Philos. Trans. Roy. Soc. London. Ser. A., 247:307-327, 1954.

[Olv3] F. W. J. Olver. Asymptotics and Special Functions. Academic Press, New York-London, 1974.

[Qi1] Z. Qi. Theory of Bessel Functions of High Rank. Ph.D. Thesis. The Ohio State University, 2015.

[Qi2] Z. Qi. On the Kuznetsov trace formula for $\mathrm{PGL}_{2}$ (C). J. Funct. Anal., 272(8):3259-3280, 2017.

[Qi3] Z. Qi. On the Fourier transform of Bessel functions over complex numbers-I: the spherical case. Monatsh. Math., 186(3):471-479, 2018.

[Qi4] Z. Qi. On the Fourier transform of Bessel functions over complex numbers-II: the general case. Trans. Amer. Math. Soc., 372(4):2829-2854, 2019.

[Qi5] Z. Qi. Cancellation in the additive twists of Fourier coefficients for $\mathrm{GL}_{2}$ and $\mathrm{GL}_{3}$ over number fields. Amer. J. Math., 141(5):1317-1345, 2019.

[Tat] J. Tate. Number theoretic background. Automorphic Forms, Representations and $L$-Functions, Part 2, Proc. Sympos. Pure Math., XXXIII, pp. 3-26. Amer. Math. Soc., Providence, R.I., 1979.

[Ter] A. Terras. Harmonic Analysis on Symmetric Spaces-Higher Rank Spaces, Positive Definite Matrix Space and Generalizations. Springer, New York, 2nd ed., 2016.

[Was] W. Wasow. Asymptotic Expansions for Ordinary Differential Equations. Pure and Applied Mathematics, Vol. XIV. Interscience Publishers John Wiley \& Sons, Inc., New York-London-Sydney, 1965.

[Wat] G. N. Watson. A Treatise on the Theory of Bessel Functions. Cambridge University Press, Cambridge, England; The Macmillan Company, New York, 1944.

[WW] E. T. Whittaker and G. N. Watson. A Course of Modern Analysis. Fourth edition. Reprinted. Cambridge University Press, New York, 1962. 